\title{
Connexins in Cardiovascular and Neurovascular Health and Disease: Pharmacological Implications
}

\author{
Luc Leybaert, Paul D. Lampe, Stefan Dhein, Brenda R. Kwak, Peter Ferdinandy, Eric C. Beyer, Dale W. Laird, Christian C. Naus,
} Colin R. Green, and Rainer Schulz

Physiology Group, Department of Basic Medical Sciences, Faculty of Medicine and Health Sciences, Ghent University, Ghent, Belgium (L.L.); Translational Research Program, Fred Hutchinson Cancer Research Center, Seattle, Washington (P.D.L.); Institute for Pharmacology, University of Leipzig, Leipzig, Germany (S.D.); Department of Pathology and Immunology, Department of Medical SpecializationCardiology, University of Geneva, Geneva, Switzerland (B.R.K.); Department of Pharmacology and Pharmacotherapy, Semmelweis University, Budapest, Hungary (P.F.); Pharmahungary Group, Szeged, Hungary (P.F.); Department of Pediatrics, University of Chicago, Chicago, Illinois (E.C.B.); Department of Anatomy and Cell Biology, University of Western Ontario, Dental Science Building, London, Ontario, Canada (D.W.L.); Cellular and Physiological Sciences, Faculty of Medicine, The University of British Columbia, Vancouver, British Columbia, Canada (C.C.N.); Department of Ophthalmology and The New Zealand National Eye Centre, Faculty of Medical and Health Sciences, University of Auckland, Auckland, New Zealand (C.R.G.); and Physiologisches Institut, Justus-Liebig-Universität Giessen, Giessen, Germany (R.S.)

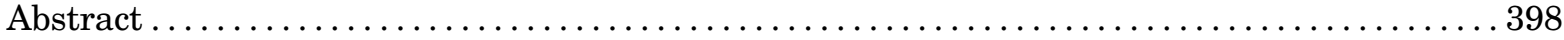

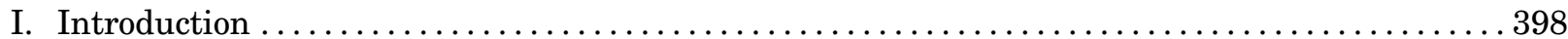

II. Expression, Life Cycle, and Regulation of Connexins.......................... 402

A. Expression of Connexins . ....................................... 402

B. The Life Cycle from Connexins to Channels and Back........................ 403

1. Connexin Trafficking and Formation of Hemichannels. .................... 403

2. Gap Junction Channel Assembly. ..................................... 404

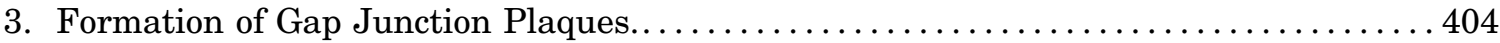

4. Gap Junction Disassembly: Internalization and Degradation. . . . . . . . . . . . 405

C. Non-Channel Functions of Connexins ................................ 406

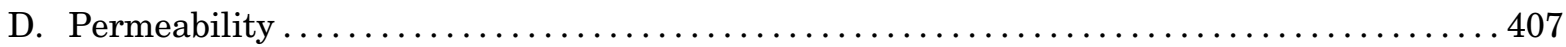

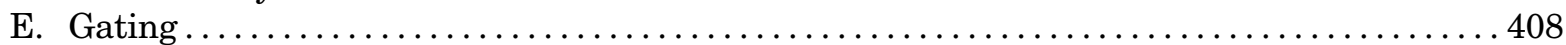

1. Slow Loop Gating and Fast Gating. .............................. 408

2. Intracellular $\mathrm{pH}$ Effects on Gating: The Role of C-Terminal Tail-Cytoplasmic

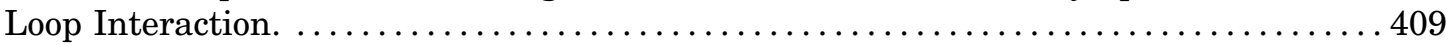

3. Voltage-Dependent N-Terminal Tail-Linked Gating. . . . . . . . . . . . . . . . . . . . . 410

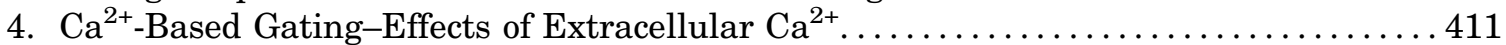

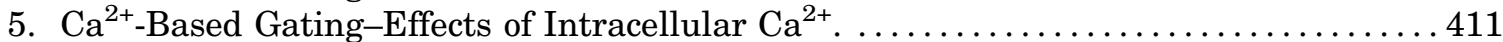
a. Intracellular $\mathrm{Ca}^{2+}$ concentration effects on gap junctions.................. 411

b. Intracellular $\mathrm{Ca}^{2+}$ concentration effects on hemichannels.................... 412

6. Other Gating Effects. ........................................... 413

F. Connexin Posttranslational Modifications............................... 413

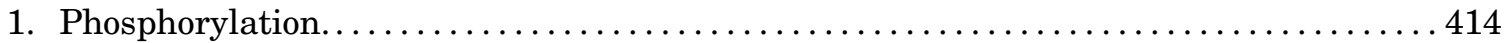

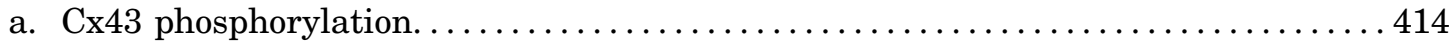

This work was supported by the Fund for Scientific Research Flanders, Belgium [Grant G.0A82.13N] to L.L.; the Interuniversity Attraction Poles Program to L.L. [Grant P7/10]; Ghent University (Bijzonder Onderzoeksfonds) to L.L.; Geneeskundige Stichting Koningin Elisabeth [Grant STI.DI2.2017.0004.01] to L.L.; the National Institutes of Health National Institute of General Medical Sciences [Grant GM055632] to P.D.L. and National Eye Institute [Grant EY08368] to E.C.B.; the Swiss National Science Foundation [Grant 310030_162579] to B.R.K.; the Canadian Institutes of Health Research [Grants 123228 and 130530] to D.W.L., the National Research, Development and Innovation Office of Hungary [Grants NVKP_16-1-2016-0017; OTKA ANN 107803, OTKA K-109737] to P.F.; and grants from the European Foundation for the Study of Diabetes (EFSD), New Horizons Collaborative Research Initiative from European Association for the Study of Diabetes (EASD), and European Cooperation in Science and Technology (COST EU-ROS) to R.S. and P.F.

P. Ferdinandy is the founder and CEO of Pharmahungary, a group of R\&D companies.

Address correspondence to: Luc Leybaert, Department of Basic Medical Sciences, Faculty of Medicine and Health Sciences, Ghent University, De Pintelaan 185, B9000 Ghent, Belgium. E-mail: Luc.Leybaert@UGent.be

https//doi.org/10.1124/pr.115.012062. 


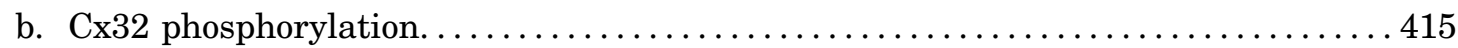

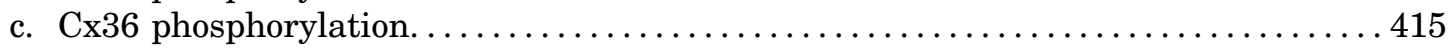

d. $\mathrm{Cx} 40$ phosphorylation. ......................................... 416

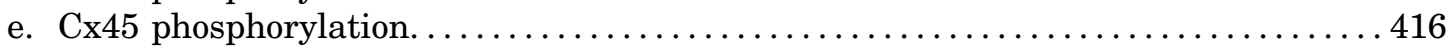

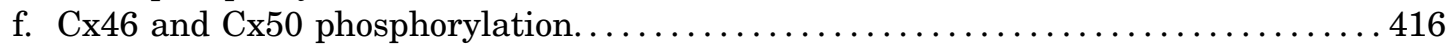

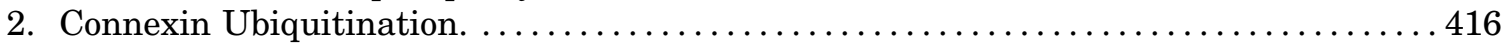

3. Other Potential Connexin Posttranslational Modifications.................... 416

4. Regulation of the Connexin Life Cycle via Posttranslational Modifications..........4 417

a. Connexin protein half-life. ................................... 417

b. Regulation of connexin export to the plasma membrane and gap junction

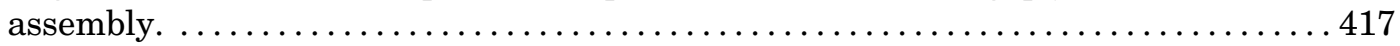

c. Regulation of gap junction turnover. .............................. 418

d. Phosphorylation effects on connexin channel gating...................... 418

III. Pharmacological Modulation of Connexin Channels ............................. 419

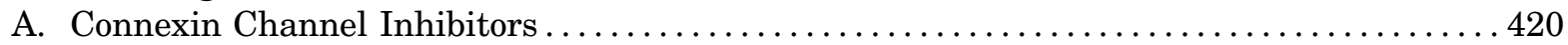

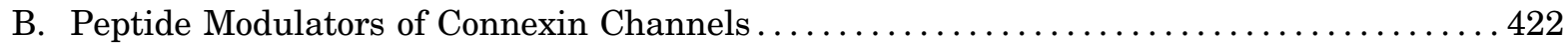

1. Mimetic Peptides of the Extracellular Loops. ........................... 422

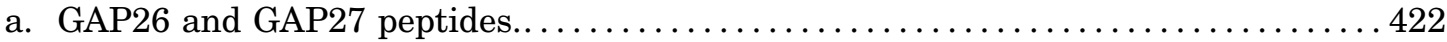

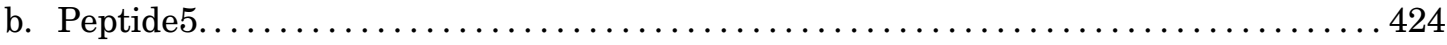

2. Mimetic Peptides of Intracellular Connexin Sequences. .................... 424

a. GAP19 and L2-specific hemichannel blocking peptides. ................. 424

2. JM2 Peptide. ................................................. 425

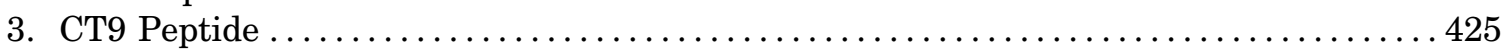

4. Note on Hemichannel-Targeting Strategies. .......................... 426

3. Peptides not Mimicking Connexin Sequences: The Case of AAP10 ............. 426

IV. Connexins in Vascular Disease ........................................ 428

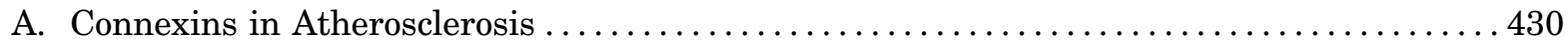

B. Connexins in Thrombosis............................................... 432

C. Endothelial Connexins in Ischemia-Reperfusion Injury $\ldots \ldots \ldots \ldots \ldots \ldots \ldots \ldots \ldots \ldots \ldots 2$

D. Connexins in Restenosis.............................................. 433

V. Connexins in Cardiac Disease ......................................... 433

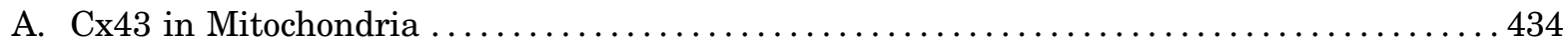

B. Cx43 and Risk Factors of Cardiovascular Diseases .......................... 435

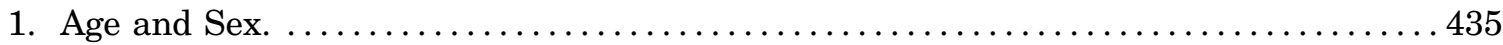

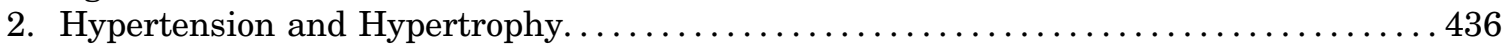

3. Diabetes and Hypercholesterolemia................................. 436

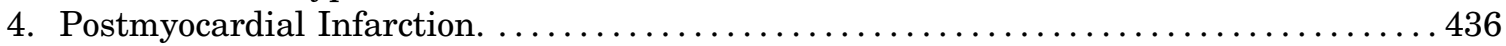

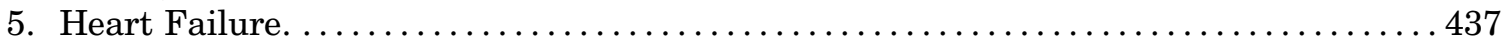

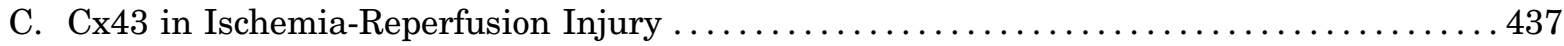

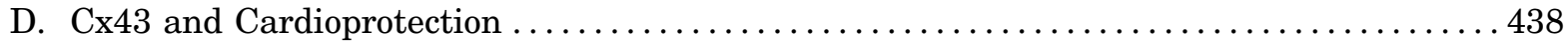

VI. Connexins and Arrhythmias ............................................ 439

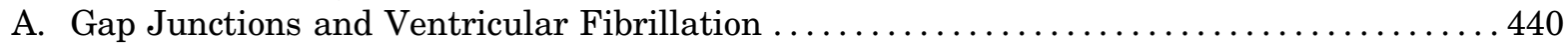

B. Gap Junctions and Atrial Fibrillation................................... 442

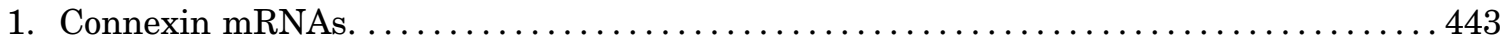

2. Connexin Protein Levels....................................... 443

3. Posttranslational Modifications of Connexins. ........................ 443

4. Connexin Distribution...................................... 443

5. Genetic Abnormalities of $\mathrm{Cx} 40$ Linked to Atrial Fibrillation. ................. 443

\footnotetext{
ABBREVIATIONS: $\mathrm{AF}$, atrial fibrillation; APD, action potential duration; $\left[\mathrm{Ca}^{2+}\right]_{\mathrm{e}}$, extracellular $\mathrm{Ca}^{2+}$ concentration; $\left[\mathrm{Ca}^{2+}\right]_{\mathrm{i}}$, intracellular $\mathrm{Ca}^{2+}$ concentration; CK, casein kinase; CL, cytoplasmic loop; CNS, central nervous system; CT, C-terminal tail; DCM, dilated cardiomyopathy; EL, extracellular loop; FGF, fibroblast gap factor; GJ, gap junction; $\gamma$, single channel conductance; HC, hemichannel; HDAC, histone deacetylase; ICM, ischemic cardiomyopathy; ID, intercalated disk; IL, interleukin; $\mathrm{IP}_{3}$, inositol, 1,4,5 trisphosphate; IPC, ischemic preconditioning; KO, knockout; LDL, low-density lipoprotein; LPS, lipopolysaccharide; MAPK, mitogen-activated protein kinase; miRNA, microRNA; MS, mass spectrometry; MM, molecular weight; NO, nitric oxide; NT, N-terminal tail; ODDD, oculodentodigital dysplasia; PKC, protein kinase C; pMCAO, permanent middle cerebral artery occlusion; ROS, reactive oxygen species; SH3, Src homology domain 3; SNP, single nucleotide polymorphism; TM, transmembrane domain; TNF- $\alpha$, tumor necrosis factor- $\alpha$; VF, ventricular fibrillation; $\mathrm{V}_{\mathrm{j}}$, junctional potential difference; $\mathrm{V}_{\mathrm{m}}$, membrane potential; ZO-1, zonula occludens 1; ZONAB, ZO-1-associated nucleic acid binding protein.
} 
6. Cx43 Mutation Linked to Atrial Fibrillation. .......................... 443

C. Further Genetic Considerations Regarding Gap Junctions and Arrhythmia ........... 444

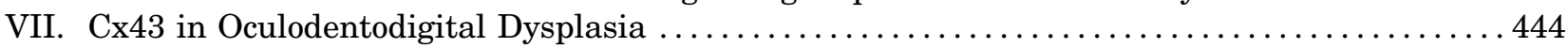

A. The Discovery of the Genetic Basis of Oculodentodigital Dysplasia ............... 445

B. GJA1 Gene Mutations Linked to Oculodentodigital Dysplasia and Beyond............446

C. Molecular Mechanisms Linking GJA1 Gene Mutations to Oculodentodigital Dysplasia... 446

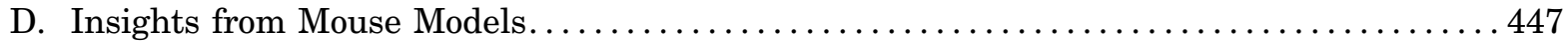

E. Next-Generation Human Models of Oculodentodigital Dysplasia................... 447

VIII. Connexins in Cerebrovascular and Retinovascular Disease........................448

A. Inflammation and Reduced Vascular Integrity in Cerebrovascular and

Retinovascular Disease............................................ 449

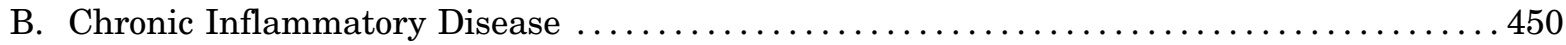

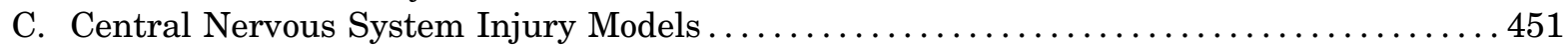

1. Brain and Spinal Cord. .......................................... 451

2. Gap Junctions, Hemichannels, and Stroke. ......................... 453

3. Ocular Models and Disease...................................... 455

4. Neurodegenerative Brain Disease................................. 457

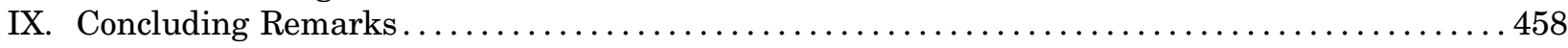

Acknowledgments..................................................... 459

References.................................................... 459

\begin{abstract}
Connexins are ubiquitous channel forming proteins that assemble as plasma membrane hemichannels and as intercellular gap junction channels that directly connect cells. In the heart, gap junction channels electrically connect myocytes and specialized conductive tissues to coordinate the atrial and ventricular contraction/relaxation cycles and pump function. In blood vessels, these channels facilitate long-distance endothelial cell communication, synchronize smooth muscle cell contraction, and support endothelialsmooth muscle cell communication. In the central nervous system they form cellular syncytia and coordinate neural function. Gap junction channels are normally open and hemichannels are normally closed, but pathologic conditions may restrict gap junction communication and promote hemichannel opening, thereby disturbing a delicate cellular communication
\end{abstract}

\section{Introduction}

Connexins are ubiquitous integral membrane proteins present in almost all cells of the body. They are strongly expressed in major organs such as the heart, brain, and liver, as well as in endothelial and smooth muscle cells of blood vessels. Their main function is to facilitate cell-cell communication and they do so in the most direct way possible, by forming channels called gap junctions (GJs) that connect the cytoplasm of cells. This short route connection serves as a powerful coordinator of cell function in complex tissues like heart and brain; it also permits efficient long-distance communication along rows of GJ-connected cells, as e.g., in the His-Purkinje conduction system in the heart or in endothelial cells of the blood vessel wall to transmit upstream vasodilatory messages (de Wit and Griffith, 2010). In electrically excitable cells like cardiac myocytes, GJ channels facilitate electrical coupling by balance. Until recently, most connexin-targeting agents exhibited little specificity and several offtarget effects. Recent work with peptide-based approaches has demonstrated improved specificity and opened avenues for a more rational approach toward independently modulating the function of gap junctions and hemichannels. We here review the role of connexins and their channels in cardiovascular and neurovascular health and disease, focusing on crucial regulatory aspects and identification of potential targets to modify their function. We conclude that peptide-based investigations have raised several new opportunities for interfering with connexins and their channels that may soon allow preservation of gap junction communication, inhibition of hemichannel opening, and mitigation of inflammatory signaling. allowing cell-to-cell passage of ions. Action potentials spread from one cell to another via GJs that are mainly localized at the cell poles in the plicate and interplicate regions of the intercalated disk (ID) (Spach and Heidlage, 1992). Isolated individual cardiomyocytes do not communicate, but when manipulated into close contact with each other, they start to communicate electrically within a couple of minutes via newly established GJ channels (Weingart and Maurer, 1988). The importance of connexins is clear from mouse knockout studies of the major cardiovascular connexins, which yield a nonviable phenotype for $\mathrm{Cx} 26^{-1-}, \mathrm{Cx} 37^{-1-}-\mathrm{Cx} 40^{-/-}$double knockouts (KOs), $\mathrm{Cx} 43^{-/-}$, and $\mathrm{Cx} 45^{-/-}$(reviewed in Simon et al., 1998; Söhl and Willecke, 2004). An example illustrating the importance of connexins in the human body concerns inherited mutations in the GJB2 gene that codes for $\mathrm{Cx} 26$, which cause congenital sensorineural deafness that has a prevalence estimated in the order of 1:5000 births (Chan and Chang, 2014; 
Esseltine and Laird, 2016). Other examples include polymorphisms of GJA4 (Cx37), which are linked to vascular disease, mutations of GJA5 ( $\mathrm{Cx} 40)$, which are known to predispose for atrial fibrillation and GJA1 mutations $(\mathrm{Cx} 43)$, which are generally not associated with a cardiac phenotype (Pfenniger et al., 2011; Delmar and Makita, 2012; Molica et al., 2014) but may lead to oculodentodigital dysplasia (ODDD), a rare primarily autosomal dominant clinical syndrome characterized by multiple malformations. An overview of connexin genes and chromosome locations can be found in Table 1 of Söhl and Willecke (2004); for the distribution of the various connexins in organs and tissues see Table 2 in Laird (2006).

GJs were discovered half a century ago (Revel and Karnovsky, 1967; Brightman and Reese, 1969), and their connexin building blocks were discovered more than 40 years ago (Goodenough, 1974). GJs are dodecameric channels formed by the interaction of two opposed hexameric hemichannels (HCs), also called connexons.
Molecular cloning studies have established that connexins form a family of related proteins (Beyer et al., 1990). Twenty-one connexin genes have been identified in the human genome and 20 in the murine genome, which encode proteins with a molecular mass (MM) that ranges from 23 to $62 \mathrm{kDa}$ (Söhl and Willecke, 2004; Beyer and Berthoud, 2009). Connexins are named according to their MM; they have a tetraspan membrane topology, with four transmembrane (TM) domains, two extracellular loops (EL1, EL2), a cytoplasmic loop (CL), and their N- and C-terminal tails (NT and CT) located inside the cell (Fig. 1). The channels formed by the different connexins often also differ in their gating properties, conductances, and permeabilities to various ions and molecules. In general, GJ channels have a pore diameter in the $10-20 \AA$ range and grant passage not only to atomic ions such as $\mathrm{K}^{+}$, $\mathrm{Na}^{+}$, or $\mathrm{Ca}^{2+}$, but also to metabolic molecules with a MM below $\sim 1.5 \mathrm{kDa}$ (assuming an approximate spherical shape) like ATP, glucose, ascorbic acid, or glutathione,

\section{Cx26}

\section{Cx43}

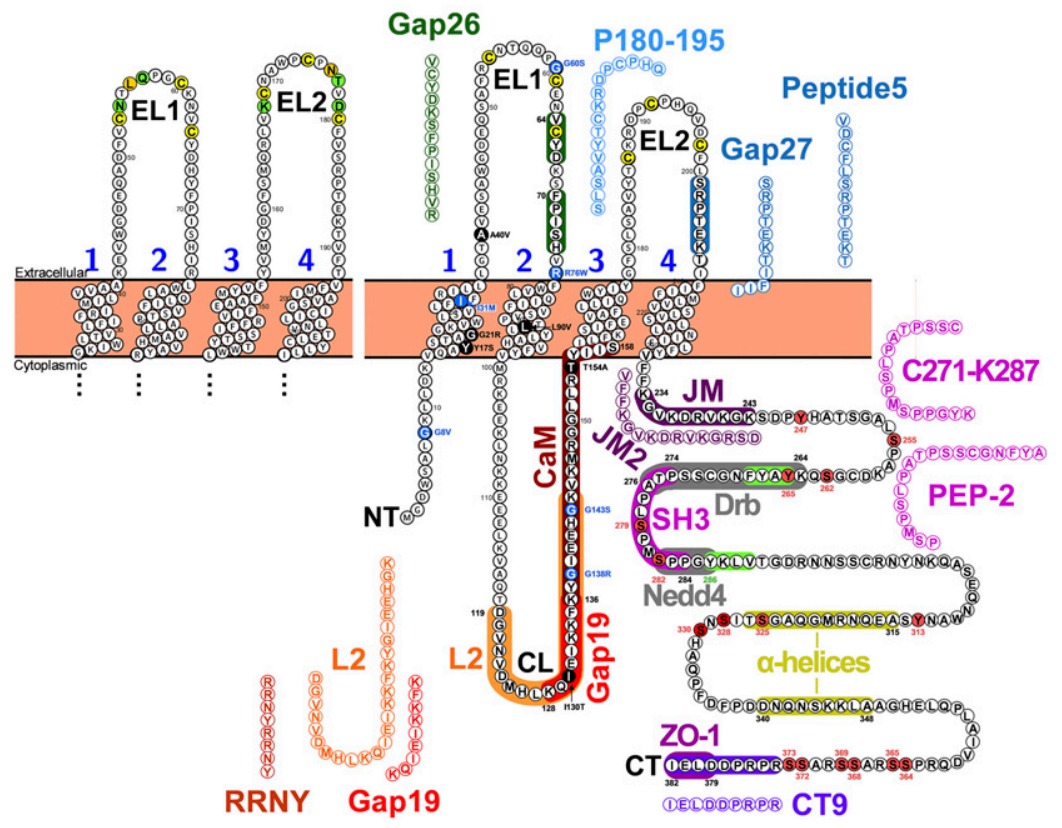

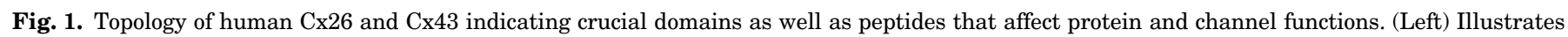

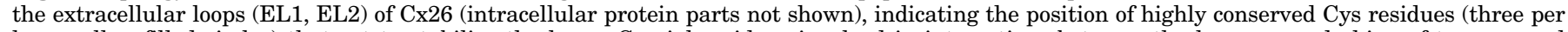

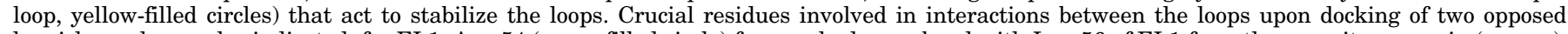

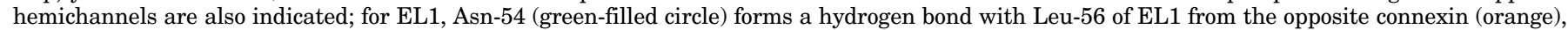

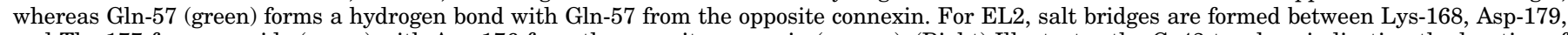

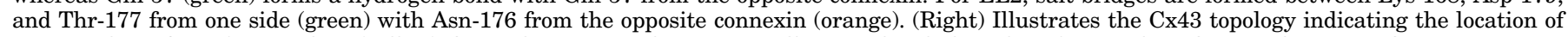

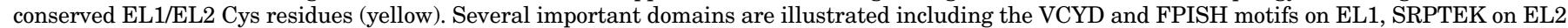

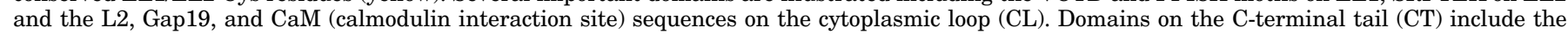

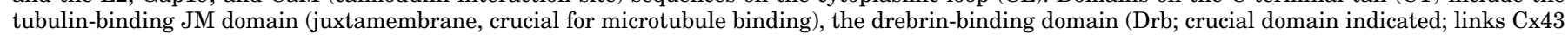

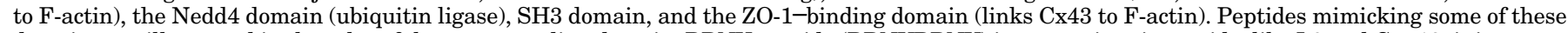

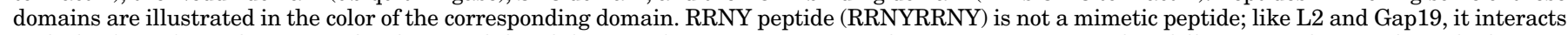

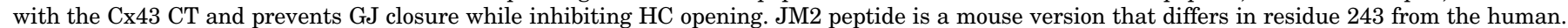

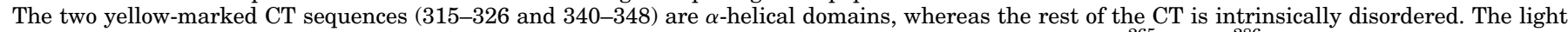

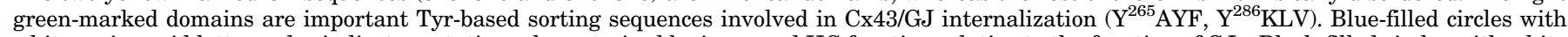

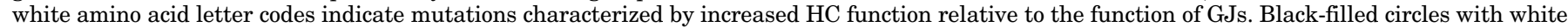

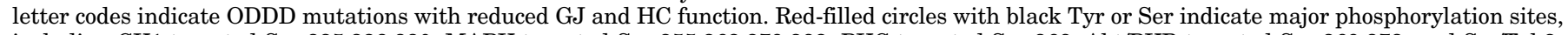

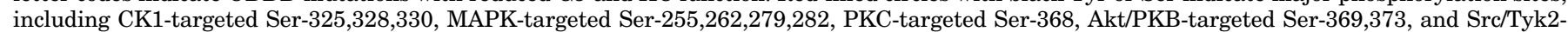

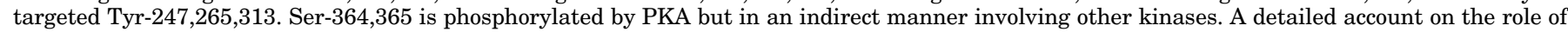
the various amino acids and domains illustrated here can be found in sections II and III. 
and second messengers such as cAMP, cGMP, or inositol trisphosphate $\left(\mathrm{IP}_{3}\right)$ (Alexander and Goldberg, 2003; Saez et al., 2003; Li et al., 2012a). Recent evidence indicates that siRNA and miRNA may also pass through GJs, most likely as a rod-shaped molecule linearly permeating the pore with its smallest dimension (Brink et al., 2012). As a result of the passage of multiple substances, which can exert beneficial but also toxic effects, GJs may also be involved in communicating and spreading harmful messages. Two marked examples of such inadequate signaling include the propagation of inflammation along the blood vessel wall (Parthasarathi et al., 2006) and bystander cell death propagation in ischemic cardiomyocytes and brain cells (Contreras et al., 2004; García-Dorado et al., 2004; Decrock et al., 2009b). GJs can close and uncouple cells in response to various conditions, either as a result of mutations or activation of signaling cascades. As illustrated above, several connexin mutations with impact on the cardiovascular system or the brain have been characterized (reviewed in Pfenniger et al., 2011; Abrams and Scherer, 2012; Delmar and Makita, 2012; De Bock et al., 2013a; Molica et al., 2014). GJs also typically close under ischemic conditions, as a result of intracellular acidification and increased cytoplasmic $\mathrm{Ca}^{2+}$ as forefront signals (Kleber, 1992; Anderson et al., 2003; Dhein, 2006a; Evans, 2015; Moore and O'Brien, 2015). Additionally, GJs are modulated in various ways by posttranslational modifications. In principle, GJs can be targeted by pharmacological inhibitors to counteract their contribution to bystander cell death, but in heart as well as in brain, the success of such an approach is not guaranteed because it will compromise the physiologic roles of GJs, i.e., impulse propagation in the heart or substrate delivery through the GJ-connected astrocytic network (Rouach et al., 2008), which also contributes, albeit partially, to spatially buffering $\mathrm{K}^{+}$away from zones of high neuronal activity (Wallraff et al., 2006). To preserve physiologic functions, GJs may be targeted to correct or prevent uncoupling. In stroke and spinal cord injury, knockout of specific connexins often gives different outcomes compared with pharmacological GJ inhibition, which may result from channel-independent connexin functions that remain operational in the latter case while absent in knockout. Another player that has entered the field and attracted considerable interest as a novel pharmacological target are the connexin HCs.

Although GJs have been investigated for over half a century, the interest in possible functions of HCs has a more recent origin. HCs were supposed to be closed until they interact and form a GJ channel at which point they open. It is only since the early 1990s that it was realized that HCs could open without necessarily forming a GJ, resulting in a conduit that communicates with the extracellular space, not with a neighbor cell (Paul et al., 1991). It is now clear that the spectrum of connexin functions encompasses actions of the connexins themselves (channel-independent functions), functions related to GJs, and functions related to $\mathrm{HC}$ opening. GJs have well established physiologic roles but they may additionally exert pathologic effects, e.g., by contributing to bystander cell death (Decrock et al., 2009b, 2017). By contrast, HCs have mainly been implicated in pathologic contexts, although it is not clear whether they have any physiologic function. HCs have roughly the same upper limit of $\sim 1.5 \mathrm{kDa}$ for passing substances but passage is predicted to be easier as the channel is half as long as a GJ channel (the conductance is twice as large for a $\mathrm{HC}$ compared with a GJ channel). HCs allow the free passage of $\mathrm{Na}^{+}$and $\mathrm{K}^{+}$, which may lead to cell swelling, and facilitate $\mathrm{Ca}^{2+}$ entry (Schalper et al., 2010; Fiori et al., 2012), which may cause cellular $\mathrm{Ca}^{2+}$ overload and promote the escape of various molecules (Chandrasekhar and Bera, 2012). These include ATP (Eltzschig et al., 2006; Kang et al., 2008; Bol et al., 2016), glutamate (Ye et al., 2003), lactate (Karagiannis et al., 2016), $\mathrm{NAD}^{+}$(Bruzzone et al., 2001), $\mathrm{IP}_{3}$ (Gossman and Zhao, 2008), $\mathrm{PGE}_{2}$ (Cherian et al., 2005; Siller-Jackson et al., 2008; Burra and Jiang, 2009), and glutathione (Rana and Dringen, 2007; Ye et al., 2015) that all potentially may become depleted when the cell is under stress and energy reserves run on empty. All released substances may additionally act as autocrine and paracrine signaling molecules, which has been best documented for ATP (Lohman et al., 2012; Wang et al., 2013b; Lohman and Isakson, 2014). HCs also facilitate molecular entry provided there is a chemical or electrochemical driving force; for example, the fluorescent glucose analog 2-( $N$-(7-nitrobenz-2-oxa-1,3-diazol-4-yl)amino)2-deoxyglucose may enter cells through HCs. Based on data from the Human Metabolome Database, Esseltine and Laird (2016) estimated the number of molecules that may be able to escape through open HCs in the order of several tens of thousands (Esseltine and Laird, 2016), underscoring the potential scope of inappropriate $\mathrm{HC}$ opening. The mechanisms that lead to HC opening link to mutational defects that result in constitutively open HCs or to triggers of electrical, chemical, or mechanical nature. Mutations may impact various levels of the connexin life cycle, including connexin transport to the plasma membrane, functions of GJs, or $\mathrm{HC}$ functions. Interestingly, some mutations result in a gain-of-function of $\mathrm{HCs}$ and a loss-of-function of GJs demonstrating that $\mathrm{HCs} / \mathrm{GJ}$, although composed of the same building blocks, can in principle be distinctly modulated. Evidence for such distinct regulation is accumulating and further expanded in this review. Some mutations of $\mathrm{Cx} 43$ that result in increased HC function, yielding leaky HCs, are illustrated in Fig. 1 (for review, see Retamal et al., 2015). In addition to constitutive mutational defects, $\mathrm{HCs}$ can be triggered to open in response to electrical signals, i.e., changes of the 
membrane potential, mechanical forces acting on the plasma membrane (Siller-Jackson et al., 2008; Batra et al., 2014), and chemical signals including the lowering of extracellular $\mathrm{Ca}^{2+}$, increases of intracellular (cytoplasmic) $\mathrm{Ca}^{2+}, \mathrm{NO}$, or more complex chemical environments such as proinflammatory conditions or ischemia-reperfusion (reviewed in John et al., 2003; Wang et al., 2013a; Castellano and Eugenin, 2014; Orellana et al., 2014, Schulz et al., 2015; Orellana, 2016).

Pannexins are another protein family composed of three members (Panx1, Panx2, and Panx3) that form channels that resemble connexin hemichannels (Bruzzone et al., 2003). They share the same tertraspan topology, with two ELs, one CL, and N- and C-terminals inside the cell, but lack sequence homology. They are vertebrate analogs of invertebrate innexins that, in contrast to innexins and connexins, generally do not form cell-cell connecting channels as GJs do. As a result, the consensus is to call hexameric channels composed of Panx1, the most common pannexin, just channels and not HCs (Sosinsky et al., 2011). Pannexins have in common with connexin HCs a wide pore, and both act as forefront diffusive ATP release pathways [estimated pore size is 17-21 $\AA$ for Panx1, slightly higher than for connexins, and $\sim 30 \AA$ for Panx2, which possibly forms octameric channels (Ambrosi et al., 2010)]. Panx1 has been demonstrated to be important in inflammasome activation (Kanneganti et al., 2007), and Panx1 and Panx2 are reported to be involved in ischemia-induced neuronal cell death [Panx1 (MacVicar and Thompson, 2010; Thompson, 2015; Weilinger et al., 2016); Panx2 (Bargiotas et al., 2011)]. Panx1-triggered ATP release acts as a find-me signal of apoptotic cells, which attracts phagocytic cells to remove dying cells early in the apoptotic process in an orderly manner (Chekeni et al., 2010). In the heart, Panx1 ATP release has been implicated in attracting phagocytes (Oishi et al., 2012) in pathologic fibrosis ( $\mathrm{Lu}$ et al., 2012a), in activating sympathetic fibers (Dong et al., 2016), in ischemic preand postconditioning (Vessey et al., 2011a,b), in atrial fibrillation (Petric et al., 2016), and as a large conductance channel in cultured atrial cardiomyocytes (Kienitz et al., 2011) (roles of cardiac pannexins are reviewed in Li et al., 2015). In blood vessels, Panx1 in smooth muscle cells is involved in the regulation of vascular tone (Billaud et al., 2012), whereas its presence in venous endothelial cells regulates leukocyte migration during inflammation (Lohman et al., 2015; reviewed in Good et al., 2015; Begandt et al., 2017).

Panx1 channels can be opened by caspases-3 and -7 , which cleave off part of the CT, and by Src family kinases that target the CT (Chekeni et al., 2010; Thompson, 2015; Weilinger et al., 2016). Most of the connexin channel inhibitors also inhibit pannexin channels (Dahl et al., 2013), but Panx1 is specifically inhibited by ${ }^{10} \mathrm{Panx} 1$, a peptide mimicking a sequence on EL1 of Panx1 (Pelegrin and Surprenant, 2006) and the quinolone antibiotic trovafloxacin, which explains the toxicity and side effects of this drug (Poon et al., 2014). Due to the lack of sequence homology with the connexins, the regulation of Panx1 channels is very different from connexins. For example, Panx1 channel open with truncation of the CT (Chekeni et al., 2010), whereas the CT is essential for $\mathrm{Cx} 43 \mathrm{HC}$ function (De Vuyst et al., 2007; Kang et al., 2008; Ponsaerts et al., 2010) (Fig. 2A). In terms of regulation by posttranslational modifications, pannexins still need to be scrutinized in detail. Most importantly, pannexins function as a plasma membrane channel without necessarily connecting cells, whereas connexins represent the more complicated case because they form junctional channels as well as HCs. For these reasons, in this review we
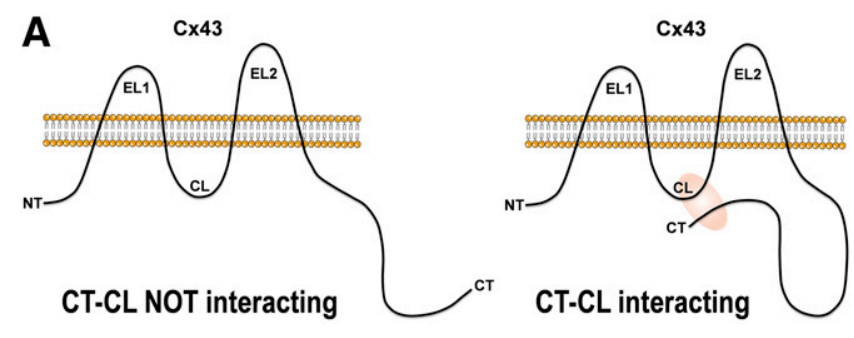

\section{Gap junctions open Gap junctions closed}

Hemichannels closed Hemichannels available to open

B
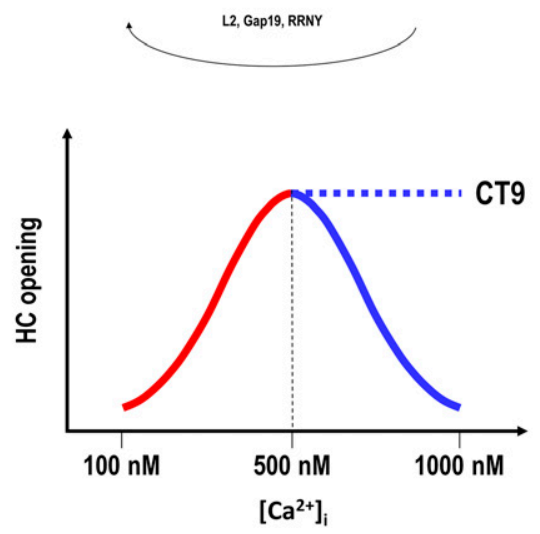

Fig. 2. Functional effects of loop-tail interactions and $\left[\mathrm{Ca}^{2+}\right]_{i}$ elevation on $\mathrm{Cx} 43$ channel function. (A) Interaction of the connexin C-terminal tail (CT) with the cytoplasmic loop (CL) distinctly influences the function of gap junctions (GJs) and hemichannels (HCs). In the absence of CT-CL interaction, GJs are open while HCs are kept closed. Upon interaction of the CT with the CL, GJs are closed while HCs become available to open. The actual opening of HCs only occurs when a trigger is present, which can be of electrical (changes in membrane potential leading to depolarization or positive voltages) or chemical nature (e.g., changes in extracellular or intracellular $\mathrm{Ca}^{2+}$ concentration, inflammatory conditions, ischemic conditions including reperfusion). HC blockers like L2, Gap19 and RRNY (sequences see Fig. 1) bind to the CT and prevent CT-CL interaction, thereby driving HCs from the available to open state to the closed state. At the level of GJs, this prevents the closure of the junctional channels. (B) $\left[\mathrm{Ca}^{2+}\right]_{\mathrm{i}}$ modulation of $\mathrm{Cx} 43 \mathrm{HC}$ opening. Moderate $\left[\mathrm{Ca}^{2+}\right]_{\mathrm{i}}$ promotes $\mathrm{HC}$ opening via calmodulin-dependent signaling (red part of the bell-shaped curve). High $\left[\mathrm{Ca}^{2+}\right]_{\mathrm{i}}$ inhibits $\mathrm{HC}$ opening by disrupting CT-CL interaction (blue part). CT9 peptide removes the high $\left[\mathrm{Ca}^{2+}\right]_{\mathrm{i}}$ brake. For details, see sections II and III. 
chose to concentrate on connexins and limit pannexin discussion to introductory concepts.

This review starts with an introduction on connexins, their expression, life cycle, and regulation (section II), followed by a more detailed account on recent insights and substances that modulate connexin channel function (section III). We next review the roles of connexins in vascular disease, including atherosclerosis, thrombosis, restenosis, and ischemia-reperfusion injury (section IV); in cardiac disease discussing risk factors, ischemia-reperfusion injury, and cardioprotection (section $V$ ); and atrial and ventricular fibrillation (section VI). Section VII gives a detailed account on GJA1 mutations that lead to the clinical syndrome of ODDD. Although a rare disease, it is a resource of mutations that affect $\mathrm{Cx} 43$ trafficking as well as channel functions, providing crucial insights into the role of specific $\mathrm{Cx} 43$ amino acid positions and domains, in some cases (e.g., I130T) leading to arrhythmogenesis. In the last chapter, we discuss the role of connexins in brain and spinal cord injury, stroke, ocular disease, and neurodegenerative brain disease, which is often associated with vascular alterations (section VIII).

Despite the various roles of connexins and their channels in physiology and pathology, the research field is handicapped because all pharmacological agents (inhibitors or promoters) to date have little specificity and often multiple side effects. However, work in the past two decades demonstrates accumulating evidence that peptide-based molecules are the better option in terms of specificity and off-target effects. Peptide studies are the perfect start and opportunity to define a pharmacophore when combined with systematic amino acid substitution approaches and with surface plasmon resonance (or its more recent congener microscale thermophoresis) and NMR studies, collectively allowing for the characterization of crucial peptide-protein interaction domains. Exemplary for such approach are the L2 and AAP peptides that counteract GJ closure, from which several interesting peptidomimetics have been developed (see section III). Another example is the CT9 peptide, which promotes the formation of GJs by affecting the $\mathrm{Cx} 43$ life cycle, and also affects $\mathrm{HC}$ function (see sections $I I$ and $I I I$ ). A second complication in the connexin field is the fact that two types of channels are formed, GJs and $\mathrm{HCs}$, the functions of which are difficult to disentangle, especially in vivo. Recently, several peptides are in the limelight, including L2 and Gap19 peptides that block $\mathrm{Cx} 43 \mathrm{HCs}$ without inhibiting GJs, and Peptide5 that inhibits HCs but not GJs when applied at low concentration (see section III). Thus, there is new excitement and expectation in the field that connexin-targeting drugs may at some point join the pharmacological arsenal available for therapeutic applications (Naus and Giaume, 2016).

\section{Expression, Life Cycle, and Regulation of Connexins}

\section{A. Expression of Connexins}

The major, most prominently expressed cardiovascular connexins are Cx37 [GJA4 gene on chromosome 1 in the human (Söhl and Willecke, 2003)], Cx40 (GJA5 on chromosome 1), Cx43 (GJA1 on chromosome 6), and Cx45 (GJA7 on chromosome 17), with $\mathrm{Cx} 43$ being the most abundant isoform. Connexins have been grouped in five families based on their sequence (conserved domains), length of the cytoplasmic loop, and gene structure, each represented by a Greek letter (Cruciani and Mikalsen, 2006; Abascal and Zardoya, 2013). $\mathrm{Cx} 37, \mathrm{Cx} 40$, and $\mathrm{Cx} 43$ belong to group $\alpha, \mathrm{Cx} 32$ is a $\beta$ connexin, and $\mathrm{Cx} 45$ is a $\gamma$ connexin. Their regional expression palette differs between the vascular system and the heart. In both, the expression pattern can vary with the species and we mainly focus on the human in what follows.

Blood vessel endothelial cells, including those of the coronary system that perfuses the heart, express $\mathrm{Cx} 37$, $\mathrm{Cx} 40, \mathrm{Cx} 43$, and $\mathrm{Cx} 45$ but the level or even presence depends on the diameter of the vessels. Cx37 and $\mathrm{Cx} 43$ are predominant at all levels up to capillaries, $\mathrm{Cx} 40$ is not present in capillary endothelial cells, and $\mathrm{Cx} 45$ is only present in large arteries. On the venous side, the connexin expression is low, and another connexin not mentioned yet, $\mathrm{Cx} 32$, is found in veins (for reviews, see Haefliger et al., 2000; Begandt et al., 2017). The smooth muscle cells surrounding the endothelium express $\mathrm{Cx} 37, \mathrm{Cx} 43$, and $\mathrm{Cx} 45$; $\mathrm{Cx} 40$ expression is low or not present.

In heart muscle, five different connexins are represented: $\mathrm{Cx} 31.9, \mathrm{Cx} 37, \mathrm{Cx} 40, \mathrm{Cx} 43$, and $\mathrm{Cx} 45 . \mathrm{Cx} 43$ is present primarily in working myocytes of the atrium and ventricle (Davis et al., 1994,1995); it is the predominant connexin in ventricular myocardium (Severs et al., 2008). It is also present in the human conduction system but less so in mice and rat (Teunissen and Bierhuizen, 2004). Cx43 also abounds in fibroblasts, which provide the structural skeleton of the myocardium that strongly expands in heart disease (McArthur et al., 2015); GJs may form between cardiomyocytes and fibroblasts, thereby increasing the arrhythmia risk. $\mathrm{Cx} 43$ is also found in stem cells used for cardiotherapeutic purposes (Lu et al., 2012b); as such, they may aid in electrically reconnecting cardiomyocytes and promote conduction (Hofshi et al., 2011). Cx40 is present in the atrium and conducting system (except in rats where $\mathrm{Cx} 43$ is the main atrial connexin) (Davis et al., 1994; Saffitz et al., 1994; Simon et al., 1998; van Rijen et al., 2001). Cx40 is prominently expressed in the ventricle early in development, but then declines to near absence in the adult (Van Kempen et al., 1996). Levels of $\mathrm{Cx} 40$ and $\mathrm{Cx} 43$ are very similar in atrial myocytes (Lin et al., 2010). Cx45 is found in many 
cardiac regions (Davis et al., 1994), but it may not be abundant; it may be important in defining the developing conduction system (Coppen et al., 1999). Cx31.9 (Cx30.2 in rodents) is a connexin that was discovered relatively recently (Belluardo et al., 2001; Nielsen et al., 2002), which may contribute to atrioventricular nodal impulse conduction (Kreuzberg et al., 2005, 2006) (human Cx31.9 gene is on chromosome 17).

Last, but not least, several types of blood cells also express connexins. Red blood cells do not express connexins but they do express Panx1 (reviewed in Begandt et al., 2017). Platelets express Cx37 and $\mathrm{Cx} 40$, with low levels of $\mathrm{Cx} 32$ and $\mathrm{Cx} 43$ and Panx1 (reviewed in Vaiyapuri et al., 2015; Molica et al., 2017). $\mathrm{Cx} 43$ and $\mathrm{Cx} 37$ are present in monocytes, and $\mathrm{Cx} 43$ is present in neutrophils and T- and B-lymphocytes (reviewed in Pfenniger et al., 2013; Glass et al., 2015).

Vascular alterations are invariably involved in diseases of the central nervous system, including stroke, traumatic injuries, and neurodegenerative disorders. The specific parenchymal connexins in neurons and glial cells are introduced in section VIII handling on the role of connexins in cerebrovascular and retinovascular disease.

Transcriptional control of $\mathrm{Cx} 40$ and $\mathrm{Cx} 43$ genes is known to be mediated by several transcription factors, including Sp1 and Sp3 for $\mathrm{Cx} 40$ and Sp1/Sp3 and AP1 for $\mathrm{Cx} 43$ (Teunissen and Bierhuizen, 2004). Cx43 transcription is promoted by tetradecanoyl phorbol acetatevia activation of c-jun and c-fos that dimerize to form AP1. Many other signals affect $\mathrm{Cx} 43$ expression including thyroid and parathyroid hormones, estrogens (see section V.B.1), prostaglandin E2, and signaling via Ras, Wnt1, and cAMP pathways. In tumor cells, connexins like $\mathrm{Cx} 43$ and others are often downregulated by hypermethylation of the gene promoters (reviewed in Vinken, 2016). Histone acetylation has the opposite effect and stimulates the expression of $\mathrm{Cx} 43$ and $\mathrm{Cx} 45$, as induced by chemical inhibitors of histone deacetylase (HDAC) enzymes such as sodium dibutyrate (Hattori et al., 2007). Other HDAC inhibitors like suberoylanilide hydroxaminic acid upregulate $\mathrm{Cx} 37$ but downregulate $\mathrm{Cx} 40$ in cardiodystrophic mice (Colussi et al., 2010). The nonspecific connexin channel inhibitor 18- $\alpha$-glycyrrhetinic acid and carbenoxolone have been reported to act via a decreased transcription (Guo et al., 1999; Herrero-González et al., 2009; Wang et al., 2009) (see section III.A).

A major point of posttranscriptional control is mediated by microRNAs (miRNAs), which may suppress translation or cleave mRNAs and thereby affect mRNA stability. For the cardiovascular connexin family, most evidence is available for $\mathrm{Cx} 43$ and $\mathrm{Cx} 40 . \mathrm{Cx} 43$ targeting miRNAs bind to the $3^{\prime}$-UTR region in the mRNA (reviewed in Vinken, 2016) and a prominent example is miR-1. When overexpressed in rat heart, miR-1 exacerbates arrhythmogenesis by reducing $\mathrm{Cx} 43$ expression as well as expression of Kir2.1 $\mathrm{K}^{+}$channel subunits (Yang et al., 2007). Interestingly, miR-1 is overexpressed in individuals with coronary artery disease, whereas it is downregulated in patients with tetralogy of Fallot (Wu et al., 2014). Other miRNAs that downregulate $\mathrm{Cx} 43$ and lead to arrhythmogenesis are miR-17-92 and miR-130a (Danielson et al., 2013; Osbourne et al., 2014) (see also section V). Some miRNAs promote connexin expression, such as miR208a that stimulates cardiac $\mathrm{Cx} 40$ expression (Callis et al., 2009). Adenylate/uridine-rich elements and RNAbinding proteins also affect mRNA stability, but they have been less characterized in a cardiovascular context (reviewed in Salat-Canela et al., 2015).

\section{B. The Life Cycle from Connexins to Channels and Back}

The connexin life cycle encompasses various steps, including 1) connexin trafficking and formation of $\mathrm{HCs}$, 2) GJ assembly, 3) formation of GJ plaques, and 4) disassembly of GJs by internalization and degradation. These various steps are introduced below; their modulation is discussed further in section II.F (for reviews, see Laird, 2006; Salameh, 2006; Thevenin et al., 2013).

1. Connexin Trafficking and Formation of Hemichannels. After biosynthesis in the ER, connexins are transported by forward trafficking to the plasma membrane via the secretory pathway involving the Golgi apparatus. Most connexins follow this pathway but trafficking for Cx26 (a connexin found in liver, cochlea, Schwann cells, and oligodendrocytes) has been reported to be Golgi dependent (Thomas et al., 2005) as well as independent (Martin et al., 2001). Hemichannels are hexameric connexin assemblies that are formed by oligomerization that starts in the endoplasmic reticulum, continues in the Golgi apparatus, and stabilizes in the trans-Golgi network (Laird, 2006). Some connexins like $\mathrm{Cx} 43$, the predominant cardiovascular connexin, and $\mathrm{Cx} 46$ (an eye lens connexin) appear monomeric in the ER/Golgi and only hexamerize in the trans-Golgi network (Musil and Goodenough, 1993; Koval et al., 1997). In Cx26, the smallest and best characterized connexin in terms of atomic structure (3.5 ̊ resolution based on X-ray crystallographic analysis), the subunit interactions that lead to oligomerization involve the outer half of transmembrane helices TM2 (a pore lining domain) and TM4 (a membrane facing domain) as well as the ELs (Maeda et al., 2009). Additionally, amino acids in the CL, close to the transition into TM3 are also involved (Smith et al., 2012). Arg-184 in Cx26 (EL2) is a crucial amino acid that, when mutated, leads to disturbed oligomerization as do mutants of Arg-75 (EL1, just before transitioning into TM2) (Maeda and Tsukihara, 2011); both Arg are well conserved in other connexins.

Vesicles containing HCs, at least those composed of $\mathrm{Cx} 43$, are transported along microtubules and actin 
filaments to the plasma membrane, and tethering of the microtubule plus ends at the adherens junction proteins promotes the plasma membrane delivery process (Shaw et al., 2007). Connexins undergo various posttranslational modifications, including extensive regulation via phosphorylation (see section II.F). Two kinases are involved in connexin trafficking, PKA and PKB (Akt kinase). Increased cyclic AMP and subsequent PKA activation with $\mathrm{Cx} 43$ phosphorylation at Ser-364 and Ser-365 promotes trafficking and hence GJ formation (Paulson et al., 2000; Shah et al., 2002); PKB/Akt phosphorylation at Ser-373 promotes trafficking through involvement of 14-3-3 proteins (Park et al., 2007) (a more detailed discussion is given in section II.F).

Once in the plasma membrane, $\mathrm{HCs}$ are kept in a closed state by two mechanisms that relate to the membrane potential and the $\mathrm{Ca}^{2+}$ concentration inside and outside the cell. HCs are typically closed at negative membrane potential $\left(\mathrm{V}_{\mathrm{m}}\right)$ as well as by the 1-2 $\mathrm{mM}$ extracellular $\mathrm{Ca}^{2+}$ concentration $\left(\left[\mathrm{Ca}^{2+}\right]_{\mathrm{e}}\right)$ (Trexler et al., 1996; Contreras et al., 2003; Verselis et al., 2009). Also in the ER, the millimolar $\left[\mathrm{Ca}^{2+}\right]$ inside keeps the HCs closed to avoid $\mathrm{ER} \mathrm{Ca}^{2+}$ store leakage. Interestingly, GJ formation discussed next is also dependent on $\left[\mathrm{Ca}^{2+}\right]_{\mathrm{e}}$ and involves E-cadherin (Jongen et al., 1991).

2. Gap Junction Channel Assembly. The next step in the connexin lifetime consists of the head-to-head docking of two HCs from adjacent cells to assemble as a GJ channel. It is currently not known whether and to what extent a separate $\mathrm{HC}$ pool exists that is not destined to become incorporated into GJs. Hemichannels on their way to GJs have been proposed to move on lipid rafts until they meet another HC to dock with Schubert et al. (2002). HC docking involves complex interactions between the ELs of the apposed proteins, resulting in the formation of a sealed conduit between the HC heads, located in the $40-\AA$-wide gap area that separates the two plasma membranes. At this occasion, the two interacting HCs open by the opening of a loop gate (Bukauskas et al., 1995; further discussed under section II.E). Based on 3.5 A resolution X-ray crystallographic data from $\mathrm{Cx} 26$ and work from other authors referred to below, the molecular details of EL interactions can be summarized as follows. First, the ELs within a HC form an anti-parallel $\beta$ sheet stabilized by three disulfide bridges between Cys residues conserved in all connexins, which connect EL1 and EL2 from each single subunit by intramolecular interactions, thereby maintaining a rigid tertiary structure that favors docking (see Fig. 9 in Foote et al., 1998; reviewed in Sosinsky and Nicholson, 2005; Yeager and Harris, 2007; Maeda and Tsukihara, 2011). Second, based on the Cx26 crystal structure, the interaction between the ELs of opposed HCs is thought to occur such that the EL protrusions from an $\mathrm{HC}$ fit into the valleys between ELs of an $\mathrm{HC}$ at the opposite side, i.e., each connexin subunit can interact with two subunits of the opposed connexon, forming a $\beta$ barrel that seals the inside of the channel from the extracellular space (Foote et al., 1998; Yeager, 1998; Yeager and Harris, 2007; Maeda et al., 2009). The inner wall of this junctional structure is formed by the interaction of opposed EL1 domains of each connexin subunit, whereas the outer wall is formed by interacting EL2 domains of each connexin (illustrated in Fig. 1 in Riquelme et al., 2013). Based on a rotation of $30^{\circ}$ between the ELs of opposed $\mathrm{HCs}$, an alternative scenario whereby EL1 interacts with an opposed EL2, and EL2 with an opposed EL1, has also been suggested (illustrated in Fig. 4 in Perkins et al., 1998). The EL sequences are highly conserved between different connexins, and data from $\mathrm{Cx} 26$ indicate that intercellular EL1-EL1 interactions involve hydrogen bond formation between a highly conserved Asn-54 at one side with Leu-56 at the opposite side, as well as between opposed Gln-57 residues. EL2-EL2 interactions involve hydrogen bonds and salt bridges formed between Lys-168, Asp-179, and Thr-177 from one side and with Asn-176 from the opposite side (Maeda et al., 2009) (Fig. 1).

3. Formation of Gap Junction Plaques. GJ channels organize in so called plaques that have a size that ranges from just visible by confocal microscopy up to several micrometers in length and contain hundreds to thousands of GJ channels (Klaunig and Shi, 2009) separated by a center-to-center distance of $\sim 10 \mathrm{~nm}$ (Goodenough and Revel, 1970; McNutt and Weinstein, 1970). Very little is known on how exactly GJ channels interact with each other to be arranged in a densely packed well-organized array of a GJ plaque (atomic force microscopy images of gap junction plaques can be found in Müller et al., 2002; Yu et al., 2007). An illdefined minimum number of channels or channels per plaque is necessary to obtain electrical or dye coupling (Palacios-Prado et al., 2009). Surprisingly, only a limited number of channels in the order of $1 \%$ are proposed to be open in a plaque [Cx36 (Marandykina et al., 2013); Cx57 (Palacios-Prado et al., 2009)]. The inclusion of newly assembled GJs in a plaque is an organized event that involves interactions with the zonula occludens 1 (ZO-1) scaffolding protein, which links $\mathrm{Cx} 43$ to F-actin. ZO-1 has three PDZ domains and $\mathrm{Cx} 43$, as most other connexins, interacts with the PDZ2 (see Thevenin et al., 2013 for more details). Exceptions are Cx36 (a connexin found in neurons and the endocrine pancreas), which interacts with the PDZ1 domain (Li et al., 2004c), Cx45 (a heart and skin connexin) that interacts with all three PDZ domains (Kausalya et al., 2001), and Cx32 (a liver connexin also found in Schwann cells), which interacts with ZO-1 domains other than PDZ or indirectly via intermediate partners (Kojima et al., 2001; Li et al., 2004b). Cx43 interacts with PDZ2 via a DLEI sequence located at the C-terminal end (see Fig. 1), with the last four amino acids being crucial; removal of the last amino acid (Ile-382) prevents the 
interaction (Jin et al., 2004). Cx43 HCs are linked to ZO-1 proteins at the periphery of a GJ plaque in a zone called the perinexus that is distinct from the centrally located "nexus" structure that contains the GJs (Rhett et al., 2011; Rhett and Gourdie, 2012). The dynamics of plaque formation is incompletely understood; it involves accretion of newly formed GJs at the outer edge of the plaque, whereas old GJs are removed from the center (Gaietta et al., 2002; Lauf et al., 2002), probably by endocytosis of small, or even large, circular double membrane vesicles called "annular junctions" or "connexosomes" (Archard and Denys, 1979; Jordan et al., 2001; Falk et al., 2009). Outer rim accretion is a twostep process whereby $\mathrm{HCs}$ assemble as GJs and GJs incorporate into plaques; the connexin-ZO-1 interaction is crucial in this process. When $\mathrm{Cx} 43$ is tagged with GFP at its CT end, a domain involved in interaction with ZO-1, GJ plaques grow significantly larger (Hunter et al., 2003). Additionally, adding a peptide composed of the last nine amino acids of the Cx43 CT (Arg-374-Ile382 further called CT9, see Fig. 1) and linked to an antennapedia internalization sequence to make it membrane permeable (called $\alpha \mathrm{CT} 1$ ) has the same plaque increasing effect (Hunter et al., 2005). This and other work from the Gourdie group, making use of various approaches, including duolink (proximity ligation) protein-protein interaction studies, demonstrates that $\mathrm{HCs}$ are linked to $\mathrm{ZO}-1$ and that disruption of this linkage is crucial in the process of HC incorporation and GJ assembly into the plaque (reviewed in Palatinus et al., 2012; Rhett et al., 2013). Given the multitude of scaffolding and cytoskeletal links to the $\mathrm{CT}$, other proteins and structures such as microtubules (Toyofuku et al., 1998; Giepmans et al., 2001) and myosin VI (Waxse et al., 2017) may also be involved. At least two kinases are involved in GJ assembly and incorporation in GJ plaques: $\mathrm{PKB} / \mathrm{Akt}$ and casein kinase (CK)1. PKB/Akt phosphorylation of Ser-372 or -373 helps in loosening the binding of $\mathrm{Cx} 43$ to ZO-1 (Chen et al., 2008); CK1 phosphorylation of Ser-325, Ser-328, and Ser-330 promotes GJ/plaque assembly (Cooper and Lampe, 2002) and promotes dye coupling and GJ channel opening (Lampe et al., 2006). Of note, ZO-1 interactions with the $\mathrm{Cx} 43 \mathrm{CT}$ are not necessary for HC/GJ formation (Fishman et al., 1991; Dunham et al., 1992). ZO-1-CT interactions are modulated by various influences: 1) c-Src interacts with the Src homology domain 3 (SH3) domain on the Cx43 CT [Pro-274-Pro-284 (Kanemitsu et al., 1997), see Fig. 1], and this displaces the ZO-1-CT interaction that is located $\sim 100$ amino acids away from the SH3 domain (Sorgen et al., 2004); 2) ZO-1-CT interactions decrease upon acidification, and this favors c-Src binding to CT (Duffy et al., 2004); and 3) ZO-1-CT interactions increase upon isolating cardiomyocytes from their native environment and are thus involved in remodeling (Barker et al., 2002).
4. Gap Junction Disassembly: Internalization and Degradation. Several possible scenarios exist for GJ removal. Early work suggested dispersal of junctional particles in the plasma membrane, away from the junction (Lane and Swales, 1980). It has also been proposed that GJs may split into reseparated HCs according to an unzippering scenario. GJ unzippering has been suggested as a mechanism for peptide inhibitors of GJ channels like Gap26 or Gap27 (see section III) (Berthoud et al., 2000), but there is currently no hard evidence to support such a mechanism. Possibly, HCs are also internalized: making use of a fluorescently labeled peptide identical to a Cx43 EL1 sequence and CFP-tagged Cx43, Dermietzel et al. (2003) demonstrated uptake of double-labeled structures, indicating inward trafficking of HCs (Dermietzel et al., 2003). However, the currently best characterized uptake scenario consists of the uptake of an entire GJ plaque (or parts of it), which then appears in one of the cells as an annular junction (Jordan et al., 2001; Piehl et al., 2007; Falk et al., 2009). The uptake process can occur by clathrin (Gumpert et al., 2008)- or caveolin-dependent (Schubert et al., 2002; Lin et al., 2003b) endocytosis (reviewed in Thevenin et al., 2013). Clathrin-dependent uptake involves several clathrin adapter proteins such as adaptor protein complex-2 that interact with Tyr-based sorting signals located on the CT tail (in particular $\mathrm{Y}^{265} \mathrm{AYF}, \mathrm{Y}^{286} \mathrm{KLV}$, and others for $\mathrm{Cx} 43$; see Fig. 1) and disabled2. Dynamin2, a GTPase, facilitates the inward pinching-off of plasma membrane vesicles, whereas myosin-VI provides vectorial inwardly directed transport. Further breakdown can occur via lysosomal or proteasomal pathways (Laing and Beyer, 1995; Laing et al., 1997,1998; Qin et al., 2003, reviewed in Salameh, 2006). Ubiquitination is central for proteasomal breakdown, but proteasomal breakdown of $\mathrm{Cx} 43$ can occur in the absence of ubiquitination (Su et al., 2010; Su and Lau, 2012); ubiquitination can furthermore be involved in endosomal/lysosomal breakdown. Ubiquitination occurs at Lys residues, of which $\mathrm{Cx} 43$ has 23; 20 of them are located on the CL and CT, which contain the more likely ubiquitination sites. The large GJ structures are not typical targets for proteasomal breakdown, but Girão et al. (2009) reported that a $\mathrm{Cx} 43$-ubiquitin fusion protein present in HCs and GJ plaques was internalized by a process that involves the ubiquitin ligase Nedd4 and the endocytic adapter protein Eps15. Nedd4 interaction with $\mathrm{Cx} 43$ occurred at a Pro-rich motif in the CT that overlaps with the Tyr-286 based sorting signal ( $\mathrm{Y}^{286} \mathrm{KLV}$ ) (Fig. 1) (Leykauf et al., 2006; Girão et al., 2009). Mutation of Tyr-286 and of downstream Val-289 increases the $\mathrm{Cx} 43$ half-life and stability of the GJs (Thomas et al., 2003; Catarino et al., 2011). Ubiquitination may also play a role in the endosomal/lysosomal pathway where it may target endosomes for lysosomal breakdown via the Tsg101 sorting protein that also binds to $\mathrm{Cx} 43$ (Auth et al., 2009; Leithe et al., 2009). In 
addition to proteasomal and lysosomal degradation, growing evidence indicates involvement of autophagic degradation of connexins as well (Lichtenstein et al., 2011; Bejarano et al., 2012; Falk et al., 2012; Fong et al., 2012; Iyyathurai et al., 2016). These various remova1/internalization pathways offer interesting, yet unexplored, handles to modulate GJ size (Beyer and Berthoud, 2002).

\section{Non-Channel Functions of Connexins}

Connexins are best known for their function as channels, GJs and HCs. However, connexin proteins are also endowed with channel-independent functions. Many, but not all, of the below described non-channel effects link to the CT tail of $\mathrm{Cx} 43$, which is a hub for interactions with other scaffolding or signaling proteins (Giepmans, 2004; Hervé et al., 2012) and also the site by preference for modulatory phosphorylation events. The list of non-channel functions includes connexin interactions with cytoplasmic signaling molecules, connexin-linked sequestering of transcription factors, CT-migration to the nucleus, and adhesion aspects linked to the mechanical cell-cell connection provided by GJs.

In terms of interaction with cytoplasmic signaling molecules, there is evidence that connexins interact with apoptotic factors thereby influencing cell death. $\mathrm{Cx} 26$ and $\mathrm{Cx} 43$ colocalize with the Bcl-2 proteins Bak, Bcl-xL, and Bax in the cytoplasm of human breast and colorectal cancer cells (Kanczuga-Koda et al., 2005a,b). Recent evidence demonstrates a more direct interaction of $\mathrm{Cx} 43$ with apoptosis signal-regulating kinase 1 (Giardina et al., 2007). Another example comes from the glioma brain tumor field. In these tumors, the levels of $\mathrm{Cx} 43$ decrease with increasing malignancy (Sin et al., 2012). The inverse relation between tumor cell proliferation and $\mathrm{Cx} 43$ expression in part links to the fact that $\mathrm{Cx} 43$, in particular the CT tail, exerts a brake on cell proliferation (Zhang et al., 2003). Work from the Tabernero group recently demonstrated that $\mathrm{Cx} 43$ inhibits proliferation by interacting with the protooncogene Tyr protein kinase c-Src via a CT-located SH3 domain (see Fig. 1), which involves corecruitment of the phosphatase and tensin homolog and CT Src kinase (González-Sánchez et al., 2016; reviewed in (Tabernero et al., 2016). Interestingly, a peptide composed of Ala-266 to Pro-283 [PEP-2 (Gangoso et al., 2014); see Fig. 1] was able to mimic the effect of the full $\mathrm{Cx} 43$ protein in limiting the proliferation glioma stem cells (González-Sánchez et al., 2016); more recent work demonstrated that PEP-2 was able to reduce migration, invasion, and survival of primary glioma stem cells isolated from human glioma tumor samples (JaraizRodriguez et al., 2017).

In terms of signaling to the nucleus, there are two possibilities: either the CT is involved in sequestering cytoplasmic transcription factors in a direct/indirect manner, or the CT itself translocates to the nucleus. The transcription factor ZONAB (ZO-1-associated nucleic acid binding protein) is an example of the first possibility. ZONAB associates, through its ZO-1 binding partner, with the glial connexins $\mathrm{Cx} 30, \mathrm{Cx} 43$, and $\mathrm{Cx} 47$ (Penes et al., 2005; Li et al., 2008b). ZONAB not linked to ZO-1 migrates to the nucleus where it acts as a transcription factor that binds to promoter sequences containing an inverted CCAAT box (Balda and Matter, 2000) and modulates the cell cycle, mostly resulting in increased proliferation (reviewed in Zahraoui, 2004). The ZONAB link to connexins and cell proliferation is thus indirect and based on evidence from the tight junction field; in any case ZONAB-sequestering by $\mathrm{Cx} 30, \mathrm{Cx} 43$ and $\mathrm{Cx} 47$ may be involved in inhibition of cell proliferation by these connexins. Next to ZO-1 sequestering of $\mathrm{ZONAB}, \mathrm{Cx} 43$ also interacts with $\mathrm{ZO}-2$ (Singh et al., 2005), which sequesters the transcription factors c-jun, c-fos and CCAAT enhancer binding protein (Betanzos et al., 2004), again resulting in decreased cell cycle activity and proliferation (Tapia et al., 2009). Another example of connexin-sequestering comes from the cell-cell adhesion molecule $\beta$-catenin. $\mathrm{Cx} 43$ interacts with $\beta$-catenin (Ai et al., 2000) and in this way influences $\mathrm{Wnt} / \beta$-catenin signaling, which acts to promote cell cycling and proliferation. Thus, $\mathrm{Cx} 43-\beta$ catenin interaction reduces $\beta$-catenin availability, inhibiting its migration to the nucleus and its subsequent interaction with transcription factors, thereby decreasing cell proliferation (MacDonald et al., 2009). Direct migration of the $\mathrm{Cx} 43 \mathrm{CT}$ to the nucleus has also been implicated in the $\mathrm{Cx} 43$-suppressive effect on cell proliferation despite the fact that the CT does not contain a known nuclear target sequence (Dang et al., 2003).

Connexins are also endowed with cell adhesion functions (Lin et al., 2002; reviewed in Prochnow and Dermietzel, 2008). The Kriegstein group demonstrated that the radial migration of excitatory neuronal precursor cells along radial glial cells during cortical development depended on adhesive properties provided by GJs composed of $\mathrm{Cx} 26$ and $\mathrm{Cx} 43$ (Elias et al., 2007). Follow up work showed this was also the case for the more complex migration pathway of inhibitory neuronal precursors, which come from lateral and make a switch in radial direction upon interacting with radial glia (Elias et al., 2010; reviewed in Elias and Kriegstein, 2008). Work from the Naus group demonstrated that adhesion-linked connexin properties also play a role in the migration and invasion of glioma cells (Sin et al., 2016; reviewed in Matsuuchi and Naus, 2013; Naus et al., 2016). Cx43-mediated adhesion effects have also been reported for the spreading of B-lymphocyte adhesion when cultured on a substrate (Machtaler et al., 2011). Forced expression of $\mathrm{Cx} 43$ in prostate cancer cells was furthermore found to promote metastasis to the bone, which strongly expresses $\mathrm{Cx} 43$ (Lamiche et al., 
2012). This suggests that the docking of two apposed $\mathrm{HCs}$ and/or the function of GJs may play a role in tumor metastasis to specific organs. Breast tumor carcinoma cells express $\mathrm{Cx} 43$ and frequently metastasize to the brain; recent work has provided evidence that breast metastatic carcinoma cells take advantage of GJ communication with $\mathrm{Cx} 43$-expressing astrocytes, demonstrating that the role of connexins in metastasis definitely involves channel functions (Chen et al., 2016).

Last but not least, alterations in all aspects of the connexin life cycle (see section II.B) may in some way be linked to non-channel effects. For example, changes in connexin expression or the presence of mutant connexins may affect the trafficking of other proteins that follow the secretory pathway and thereby influence intracellular vesicle trafficking and the release of exosomes, which also contain $\mathrm{Cx} 43$ (Soares et al., 2015). A striking example comes from the heart, where the loss of $\mathrm{Cx} 43$ expression leads to reduced $\mathrm{Na}^{+}$ currents in ventricular (Danik et al., 2008; Jansen et al., 2012) and atrial cardiomyocytes (Desplantez et al., 2012a). Similar findings of a reduced $\mathrm{Na}^{+}$ current, further complicated by ventricular fibrillation, were observed in a cardiac knock-in model of $\mathrm{Cx} 43$ that lacked the last five amino acids in the CT [Cx43D378stop mutation (Lübkemeier et al., 2013)]. Work of the Delmar group in this model demonstrated that, although GJ coupling is normal, $\mathrm{Na}_{\mathrm{V}} 1.5$ delivery to the plasma membrane was impaired in a microtubuledependent manner (Agullo-Pascual et al., 2014b). Omission of the five last Cx43 CT amino acids was found to limit the capture of the microtubule plus end tracking protein end-binding- 1 at the ID, resulting in impaired cargo delivery to this location (reviewed in Leo-Macias et al., 2016).

Further reviews on non-channel functions of connexins can be found in Giepmans (2004), Jiang and Gu (2005), Dbouk et al. (2009); Hervé et al. (2012), Vinken et al. (2012), Zhou and Jiang (2014); Table 1 of Zhou and Jiang (2014) gives an overview of channel-independent effects.

\section{Permeability}

The structure of HCs and GJ channels composed of Cx26 has been studied in much detail, making use of three-dimensional X-ray crystallographic analysis at $3.5 \AA$ resolution (Maeda et al., 2009; reviewed in Maeda and Tsukihara, 2011). These studies suggested that the smallest pore diameter of the $\mathrm{Cx} 26$ channel is $\sim 14 \AA$. As a consequence, $\mathrm{Na}^{+}, \mathrm{K}^{+}$, and $\mathrm{Cl}^{-}$can pass through in the presence of their hydration shell [diameters 3.58, 3.31, and $3.32 \AA$, respectively (Volkov et al., 1997)]. The lowpass cut-off for a 14- $\AA$-wide pore is in the order of $\sim 1.2 \mathrm{kDa} \mathrm{MM}$ based on an empirically derived relation between diameter and molecular weight for globular proteins (Erickson, 2009). Based on size exclusion studies with Alexa dyes, the pore diameter of $\mathrm{Cx} 43$ channels was estimated to be in the order of $14.8 \AA$ (Weber et al., 2004), giving a calculated $\sim 1.4 \mathrm{kDa} \mathrm{MM}$ cut-off. For Cx37, size cut-off based on polyethylene glycols was in the 6.8-8 A range (Gong and Nicholson, 2001). In addition to size, permeation also depends on charge. Although the narrowest diameter of the pore is much wider than hydrated $\mathrm{Na}^{+}$or $\mathrm{Cl}^{-}$, the permeability for these ions can be different, indicating charge selectivity, even for atomic ions (Veenstra et al., 1995). For larger charged molecules, which are more likely to interact with the channel wall, charge selectivity is even more pronounced and sometimes very different between different connexins. For example, negatively charged molecules like carboxyfluorescein $(8.2 \AA$, charge -2$)$ or Lucifer yellow (9.9 A, charge -2) permeate better through GJ channels composed of $\mathrm{Cx} 43$ than those composed of $\mathrm{Cx} 40$. By contrast, positively charged molecules like ethidium bromide $(10.3 \AA,+1)$ permeate better through Cx26-based channels (Kanaporis et al., 2011). Despite the importance of size and charge, there are no obvious rules to explain the permeation of metabolic or signaling molecules, suggesting that still unknown interactions (electrostatic, van der Waals, or covalent in nature) occur within the channel pore (Weber et al., 2004; reviewed in Harris, 2007). As a result, channel permeation does not occur along a free diffusion scenario but based on selectivity properties that are still poorly understood (Ek-Vitorin and Burt, 2013). For example, Cx26 channels are more or less equally permeable to cAMP (MM 329) and $\mathrm{IP}_{3}(\mathrm{MM} 420)$ despite the large difference in charge (neutral and -6 , respectively) (Hernandez et al., 2007). ATP (MM 507, charge -4 ) permeates a 300 -fold better through $\mathrm{Cx} 43$ channels than through Cx32 channels (Goldberg et al., 2002), a huge difference probably difficult to explain on pore size only. For cAMP, the permeation sequence is $\mathrm{Cx} 43>\mathrm{Cx} 26>\mathrm{Cx} 45=\mathrm{Cx} 32$ (Wang and Veenstra, 1997; Bedner et al., 2003, 2006). Additionally, the permeability for fluorescent dye molecules does not always go hand in hand with the channel electrical conductance properties (fully open state versus substrates; see section II.E) (Brink et al., 2006; Eckert, 2006) and high conductance channels like those formed by $\mathrm{Cx} 37$ display poor permeation of fluorescent dyes (Veenstra et al., 1994, 1995). Clearly, connexin channels display connexin-specific permeability profiles, and more studies are needed to have a better understanding of both the exact pore size (based on structural and functional approaches) and the nature of the interactions of the permeant with the channel. This is especially relevant for the larger permeating substances that are more likely to interact with the channel pore-lining TM1 and TM2 domains compared with atomic ions that have more space available and therefore are able to diffuse more freely with less interactions with the channel walllining residues; when the diameter of the permeant comes in the range of the pore diameter, interactions 
with the channel wall will rather determine the permeability profile.

Connexin channels can also be formed by different connexins, resulting in heteromeric or heterotypic channels. HCs can be homomeric or heteromeric (composed of different connexins). Two homomeric HCs make a homotypic GJ channel. A heterotypic GJ channel can be formed by two HCs each composed of a different connexin or by two heteromeric HCs. Two heteromeric HCs may also form a homotypic GJ channel when each subunit pairs with a subunit composed of the same connexin isoform (reviewed in Koval et al., 2014; the various configurations are illustrated in Fig. 1 of Mese et al., 2007). The most studied heteromeric/ heterotypic GJs are formed by the cardiovascular connexins $\mathrm{Cx} 37, \mathrm{Cx} 40, \mathrm{Cx} 43$, and $\mathrm{Cx} 45$, which can form heteromeric (He et al., 1999; Beyer et al., 2013) and heterotypic GJs (Lin et al., 2014). The compatibility of different connexins to form heteromeric/heterotypic channels is determined by motifs located at the CL-TM3 transition and left half of EL2. Importantly, heteromericity largely influences GJ permeability for metabolites or signaling molecules, thereby allowing the fine-tuning of the repertoire of substances exchanged between cells (Harris, 2007). Interestingly, heterotypic channels display electrical rectification behavior that may facilitate transport and electrical conduction in a preferential direction (Rackauskas et al., 2007; reviewed in Harris, 2002).

Connexin channels are often called aqueous pores, meaning they allow ions in aqueous solution to pass through the pore surrounded by their hydration shell, as mentioned earlier. In principle, water is able to pass through the channel but only little information is currently available demonstrating direct water flow through the channels. In the lens, GJs composed of $\mathrm{Cx} 46$ and $\mathrm{Cx} 50$ have been reported to be involved in water flow (Gao et al., 2011). Work on HCs seemed to confirm this, based on cell swelling observed in cell expression systems [Cx43 (Quist et al., 2000); $\mathrm{Cx} 46$ (Paul et al., 1991)]. However, more detailed analysis performed on $\mathrm{HCs}$ composed of $\mathrm{Cx} 30$ or $\mathrm{Cx} 43$ has demonstrated they are not water permeable (Hansen et al., 2014). Taken together, connexin channels may be involved in water fluxes, but this is probably the result of ionic flow through GJs or HCs with subsequent osmotic water flow through other pathways, e.g., via aquaporins.

\section{E. Gating}

Connexin channels are modulated by numerous influences that link to the cell state or internal/external influences, which most commonly link to voltage, $\mathrm{pH}$, $\mathrm{Ca}^{2+}$ concentration, phosphorylation state, and redox state. Several conditions, especially inflammatory conditions, strongly affect connexin channels. Connexin channel permeability expresses the degree of permeation of noncharged permeants, whereas conductance expresses the degree of permeation of charged permeants, i.e., the ease of current passage. Macroscopic conductance is the conductance of an array of channels; modulatory influences alter the macroscopic conductance, which can be caused by changes in the number of channels present, the conductance of single channels, or their gating, i.e., opening and closing activities. Single channel conductance $(\gamma)$ and gating properties are determined in voltage-clamp experiments, dual cell voltage-clamp for GJ channels and single cell patch-clamp experiments for HCs. These experiments further bring up important information on other biophysical properties like the rise time for opening/closing transitions and the reversal potential, i.e., the potential at which the current reverses direction. Because connexin channels have no selectivity for atomic ions like $\mathrm{Na}^{+}, \mathrm{K}^{+}, \mathrm{Ca}^{2+}$, and $\mathrm{Cl}^{-}$, the reversal potential is in most cases $\sim 0 \mathrm{mV}$. The single channel conductance is a fundamental property that differs among the different members of the connexin protein family; hence it can be used to determine what connexin is recorded from (an overview of single channel conductances of HCs and GJs is given in Table 3 of Sáez et al., 2005). Below follows a brief overview on the gating of GJs and HCs by voltage, $\mathrm{Ca}^{2+}$, and $\mathrm{pH}$. Connexin channel gating is a very complex field, in part because the gating, as well as other biophysical properties, differ substantially between different connexins. For that reason, observations in channels composed of $\mathrm{Cx} 26, \mathrm{Cx} 32, \mathrm{Cx} 45$, or $\mathrm{Cx} 46$ to name some, are not necessarily true for $\mathrm{Cx} 43$, which we keep as the prototypic example case for this review. Excellent entries into the connexin gating subject can be found in Bukauskas and Verselis (2004), Sáez et al. (2005), González et al. (2007), Moreno and Lau (2007), Ek-Vitorin and Burt (2013), Fasciani et al. (2013), Oshima (2014), and Oh and Bargiello (2015). What follows below is an overview of the most salient aspects of gating, highlighting some proposed structural correlates.

1. Slow Loop Gating and Fast Gating. HCs are normally closed but open upon docking with an apposed HC by a process of loop gating (Bukauskas et al., 1995; Trexler et al., 1996; Contreras et al., 2003), which involves movements of residues at the border of TM1/EL1 [in particular at position $43-50$ for Cx50 (Verselis et al., 2009)] and is characterized by slow gating (transition time $\geq 10 \mathrm{~ms}$ ) (reviewed in Bukauskas and Verselis, 2004). Loop gating involves large conformational changes, in particular in the first half of EL1 (Bargiello et al., 2012). Loop gating is associated with various aspects, including 1 ) the docking process of two $\mathrm{HCs}$ per se, 2) the change in voltage sensed by the two HCs upon assembling into a GJ, and 3) the change in $\left[\mathrm{Ca}^{2+}\right]$ at the external side of the HC upon GJ assembly. The first aspect links to the 
interaction of the ELs during docking, which can be seen as ligand-induced gating (Trexler et al., 1996). The second aspect links to the fact that the negative $V_{m}$ sensed by the HCs, which keeps them closed, disappears upon formation of a GJ channel between two cells with equal (or approximately equal) $\mathrm{V}_{\mathrm{m}}$. The third aspect links to the lowering of $\left[\mathrm{Ca}^{2+}\right]$ sensed by the outer half of the HC upon docking and sealing-off the channel interior from the extracellular space where $\left[\mathrm{Ca}^{2+}\right]$ is in the millimolar range (see more in section II.E.4). GJs start to close (with slow kinetics taking hundreds of milliseconds to seconds) when the junctional potential difference $\left(V_{j}\right)$, i.e., the difference in $V_{m}$ between the cells connected by the GJ, starts deviating from $0 \mathrm{mV}$ (reviewed in Bukauskas and Verselis, 2004; PalaciosPrado and Bukauskas, 2012). As a result, the relation between $V_{j}$ (abscissa) and GJ macroscopic current (ordinate) is a convex-up bell-shaped curve centered around the ordinate, with channel closing at strongly negative or positive voltages (see Fig. 2 in Bukauskas and Verselis, 2004). Single channel analysis has demonstrated that $\mathrm{V}_{\mathrm{j}}$ closure of GJ channels is, in contrast to slow loop gating, mediated by fast gating (transition time $<2 \mathrm{~ms}$ ) by a gate that is different from the loop gate (Bukauskas and Verselis, 2004). Slow gating is also involved in the closing process, in particular at the top of the bell-shaped GJ voltage dependence curve (around $\mathrm{V}_{\mathrm{j}}=0 \mathrm{mV}$, where gating starts closing fully open GJ channels) as well as at edges where full closure of the channels is at stake (see Fig. 2 in Bukauskas and Verselis, 2004). As alluded to above, HCs not incorporated into GJs can be opened by depolarizing or positive membrane potentials (Sáez et al., 2005); HC gating also displays slow and fast transitions just like GJs (Contreras et al., 2003). The majority of the gating state transitions of GJs/HCs is slow and only transitions to a residual (substrate) conductance, characterized by a $\gamma$ that is lower than the fully open channel $(\sim 60 \mathrm{pS}$ for Cx43 HCs), are fast. The slow gate always opens with depolarizing or positive voltages $\left(\mathrm{V}_{\mathrm{m}}\right)$; the fast gate opens with positive $\mathrm{V}_{\mathrm{m}}$ for $\mathrm{Cx} 32$ but closes with positive $\mathrm{V}_{\mathrm{m}}$ for $\mathrm{Cx} 26$. As a result, Cx32 HCs increase their open probability with increasing positive voltages; in contrast, $\mathrm{Cx} 26 \mathrm{HCs}$ open with positive voltages but start to close again at increasing positivity, displaying a typical bipolar voltage-dependence (González et al., 2007; Fasciani et al., 2013). Cx43 HCs reportedly also display a bipolar voltage-dependence; however, although slow gate opening clearly increases with above threshold positive membrane potentials, the behavior of the fast gate is more difficult to study because of the paucity of closing events to the residual state.

At the electrical level, GJs behave, at least in a first approximation, as a linear assembly of two apposed $\mathrm{HCs}$; their conduction is half the conduction of the corresponding $\mathrm{HC}$ and the gates appear to retain their fast and slow gating polarities in GJs as in $\mathrm{HCs}$
(González et al., 2007). A factor that complicates things is the fact that the electrical field sensed by each of the two $\mathrm{HCs}$ in a GJ depends on the closed/open state of these HCs (Paulauskas et al., 2012). Of note, GJs do not always behave as a superposition of two HCs, and the responses of both channel types to chemical signals can sometimes be opposite, as discussed below in the context of $\left[\mathrm{Ca}^{2+}\right]_{i}$ influences on channel gating. The subconductance state is considered the ground state for electrical gating mediated by fast gating (Valiunas et al., 1997; Ek-Vitorin and Burt, 2013). The ground state for "chemical gating" is the fully closed state and transitions to the fully open state are characterized by slow gating (Ek-Vitorin and Burt, 2013). Two of the most studied examples of chemical gating concern the influence of $\mathrm{pH}$ and $\mathrm{Ca}^{2+}$ on GJs/HCs, discussed below.

2. Intracellular $p H$ Effects on Gating: The Role of C-Terminal Tail-Cytoplasmic Loop Interaction. GJs close with intracellular acidification ( $\mathrm{pH}$ 5-6), as occurs in the context of ischemia. Interestingly, when the CT of $\mathrm{Cx} 43$ is truncated at Ala-257 (last 125 amino acids, including Ala-257), GJ closure upon acidification disappears (Liu et al., 1993). Moreover, re-expression of the truncated CT part rescues $\mathrm{pH}_{\mathrm{i}}$ sensitivity, indicating involvement of the truncated CT part in channel closure (Morley et al., 1996). Based on these observations, a model of Cx43 CT-CL interaction was proposed whereby the CT binds to a receptor structure, the L2 domain located in the second (CT directed) half of the CL (Asp-119 to Lys-144; Fig. 1). This intramolecular interaction was proposed to close the channel according to a ball-and-chain scenario, analogous to the ball-andchain mechanism for the closure of $\mathrm{Na}^{+}$and $\mathrm{K}^{+}$channels. The binding of the CT to the L2 domain is facilitated under low $\mathrm{pH}$ conditions, as a result of a higher $\alpha$-helical order in the L2 domain (Duffy et al., 2002); thus, CT-CL interaction and its consequences are strengthened by acidosis. Interestingly, CT truncation at residue 257 removed the residual state of the channel, suggesting that the ball-and-chain CT-CL interaction may act as a fast gate (Moreno et al., 2002). Even more interesting were the observations that addition of the L2 domain as an exogenous peptide decreased the transitions from the fully open state to the residual state and increased the open time of the GJ channels (Seki et al., 2004). Mutation of His-142, located in the L2 domain, to Glu-142 has a similar effect (Shibayama et al., 2006a). Thus, intramolecular CT-CL interactions close GJs upon acidification and addition of the L2 peptide prevents this closure. Substantial data are available in terms of the location of the CT domains that interact with the L2 domain. Morley et al. (1997) reported (based on deletion studies) that the regions Cys260-Asn-300 and Arg-374-Ile-382 from the CT domain were crucial (Morley et al., 1997) (Arg-374-Ile-382 corresponds to CT9, see Fig. 1). Follow up work demonstrated that a peptide composed of Cys-271-Lys-287 
(called C271-K287 peptide in Fig. 1) could prevent acidification-induced uncoupling (Calero et al., 1998). Duffy et al. (2002) showed that three CT peptides with sequences Cys-271-Lys-287, Asp-336-Gly-350, and Lys346-Asp-360 (mouse sequences) all interacted with L2 in surface plasmon resonance experiments (Duffy et al., 2002). The first two of these peptides, respectively, cover the SH3 domain (Pro-274-Pro-284) and an $\alpha$-helical domain (Asp-340-Ala-348); the third one is situated between the $\alpha$-helical domain and the CT9 sequence (see Fig. 1 for the location of these domains). Of note, the $\mathrm{Cx} 43 \mathrm{CT}$ contains two $\alpha$-helical domains, Ala-315Thr-326 and Asp-340-Ala-348 (illustrated as two yellow colored domains in Fig. 1), with the rest of the CT being an intrinsically disordered structure (Sorgen et al., 2004). Combined surface plasmon resonance and NMR evidence has indicated that CL interaction with the CT involves the second $\alpha$-helical domain and a region of the last 19 amino acids of the CT that includes the CT9 domain (Hirst-Jensen et al., 2007). In line with this, RXP-E peptide, discovered through a phage display search for high affinity $\mathrm{Cx} 43 \mathrm{CT}$ binders, was reported to interact with residues $343-346$ (within the second $\alpha$-helical CT domain) and 376-379 (within the CT9 domain; see Fig. 1); this peptide partially prevented acidification- and octanol-induced GJ closure (Shibayama et al., 2006b).

Evidence for involvement of CT-CL interaction in connexin channel gating is also available for $\mathrm{Cx} 46$, $\mathrm{Cx} 40$, and $\mathrm{Cx} 26$. Recent evidence indicates that the G143R mutation in the second CT-directed half of the Cx46 CL acts to strengthen CT-CL interaction (Ren et al., 2013); this results in closure of GJs and opening of $\mathrm{HCs}$, in line with the findings of CT-CL effects on GJs and HCs composed of $\mathrm{Cx} 43$ (for details on CT-CL effects on HCs see section II.E.5). For Cx40, CT truncation at residue 248 resulted in the disappearance of the residual conductance (Anumonwo et al., 2001), an effect that is similar to what is found in CT-257 truncated $\mathrm{Cx} 43$. Additionally, the $\mathrm{Cx} 43-\mathrm{CT}$ was able to substitute for the $\mathrm{Cx} 40-\mathrm{CT}$, indicating that heterodomain interactions between $\mathrm{Cx} 40$ and $\mathrm{Cx} 43$ (two prominent vascular connexins) are also possible (see also Bouvier et al., 2009). Experiments with CT-257 truncated Cx43 further suggest that CT-CL interaction is also involved in GJ inhibition by insulin and insulin-like growth factor (Homma et al., 1998). Additional evidence is available for GJ inhibition by v-Src, where CT truncation at residue 245 was most potent in removing GJ inhibition (Zhou et al., 1999). CT-CL interaction is also involved in $\mathrm{HC}$ regulation by $\left[\mathrm{Ca}^{2+}\right]_{\mathrm{i}}$ (see section II.E.5).

For Cx26, which has a very short CT (10 vs. 150 amino acids for $\mathrm{Cx} 43$ ), things are different. Locke et al. (2011) reported CT-Cl interactions at normal $\mathrm{pH}$; upon acidification, protonated aminosulfonates like taurine interact with the CL (second CT-directed half) and thereby disrupt the CT-CL interaction (Bennett, 2011;
Locke et al., 2011). Thus, opposite to $\mathrm{Cx} 46, \mathrm{Cx} 43$, and $\mathrm{Cx} 40$, for $\mathrm{Cx} 26$ it is rather a disruption of CT-CL interaction that acts to close the channels, both GJs and $\mathrm{HCs}$.

Some final remarks to conclude this part on CT-CL interaction and $\mathrm{pH}$ effects. As mentioned earlier, CT-CL interactions link to fast gating transitions to the subconductance state, which stands in contrast to the fact that chemical gating is proposed to be mediated by slow gating. It needs to be added that issues concerning the identity and functions of slow and fast gates are not unequivocally set. Additionally, GJ closure with acidification is not the prerogative of CT-CL interactions; direct effects mediated by His residues have also been reported [e.g., His-95 in TM2 of Cx43 (Ek et al., 1994)]. Another possibility for GJ closure relates to the fact that protons can displace $\mathrm{Ca}^{2+}$ from common binding sites, thereby indirectly closing the GJs by an increase of $\left[\mathrm{Ca}^{2+}\right]_{\mathrm{i}}$ (see Peracchia, 2004 and section II.E.5). Of note, a low $\mathrm{pH}_{\mathrm{i}}$ inhibits both GJs and HCs (Spray et al., 1981; Trexler et al., 1999) and there is also evidence that high $\mathrm{pH}_{\mathrm{e}}$ promotes $\mathrm{HC}$ opening (Schalper et al., 2010). As always, exceptions exist and acidosis due to moderate acid load was recently reported to increase rather than suppress GJ coupling (Swietach et al., 2007).

\section{Voltage-Dependent N-Terminal Tail-Linked} Gating. Just as the CT plays an important role in connexin channel gating, the NT domain also plays a forefront role. It has been known from 20 years of work from the Bargiello group that the NT, which is very short ( $\sim 20$ amino acids for $\mathrm{Cx} 26, \sim 22$ for $\mathrm{Cx} 32$, and $\sim 13$ for $\mathrm{Cx} 43$ ), is involved in $\mathrm{V}_{\mathrm{j}}$ voltage sensing (Verselis et al., 1994; Oh et al., 2000; Purnick et al., 2000). Especially position 2 as well as amino acids at the TM1/EL1 border appear to be crucial for voltage sensing in both $\mathrm{Cx} 26$ and $\mathrm{Cx} 32$ (for $\mathrm{Cx} 26$ crucial amino acids are Asp-2 and Lys-41 at TM1/EL1). Work from these authors demonstrated that NT-linked gating involved switching between open and a subconductance state and suggested that the NT moves toward the cytoplasm upon HC closure (Verselis et al., 1994). Moreover, it was concluded that NT movement of a single subunit was sufficient to switch to the subconductance state $(\mathrm{Oh}$ et al., 2000), which is in contrast to the cooperative tilting model whereby all subunits move in a concerted manner to open or close the channel (Unwin and Ennis, 1984). Later work by Maeda et al. (2009) based on $3.5 \AA$ crystal structure of $\mathrm{Cx} 26$ demonstrated that the NT makes a bend into the cytoplasmic side of the $\mathrm{HC}$ and is kept there in a stable position by an interaction that involves Met-34 in TM1 and others in TM1, first half of EL1, and TM2 (Maeda et al., 2009; Harris and Contreras, 2014). This particular "attached" configuration of the NT is hypothesized to determine the $14 \AA$ pore diameter of the open channel. When the voltage-sensing amino acids (especially Asp-2 and Lys-41 for Cx26) are exposed to a large junctional voltage difference, the 
NT-attachment in the pore wall is destabilized, causing NT movement toward the cytoplasm and narrowing down of the pore to a subconductance state.

Both CT-CL and NT-linked gating has been proposed to be involved in gating but from different contexts that link to chemical ( $\mathrm{pH})$ and voltage gating, respectively. It is currently unestablished whether NT- and CT-linked gating have something in common; in that regard, the CL has been proposed as a candidate interaction target for both CT and NT (see e.g., Fig. 14 in Harris, 2001). Another question is how the voltage-sensing amino acids in NT/TM1/EL1 CT could influence CT-CL interaction. Of note, the voltage-sensing domain of the slow gate is currently unidentified. Clearly, connexin channels do not have a defined and unique voltagesensor domain like the $\mathrm{S} 4$ domain of $\mathrm{Na}^{+}$channels, where four or more positively charged amino acids (Arg or Lys) are each separated by two non-charged amino acids (see Fig. 2 in Moreau et al., 2014). Likewise, the gates of connexin channels are not separate units clearly distinguishable from the voltage sensor (Harris and Contreras, 2014), and it looks like connexins combine their voltage sensing, gating, and perhaps also permeation conduit functions in multipurpose domains.

4. $\mathrm{Ca}^{2+}$-Based Gating-Effects of Extracellular $\mathrm{Ca}^{2+}$. Plasma membrane HCs not incorporated into GJs are kept in a closed state by several mechanisms that include absence of loop interactions with opposed HCs, the negative $\mathrm{V}_{\mathrm{m}}$, and the $1-2 \mathrm{mM}$ extracellular $\mathrm{Ca}^{2+}$ concentration $\left(\left[\mathrm{Ca}^{2+}\right]_{\mathrm{e}}\right.$ ) (reviewed in Fasciani et al., 2013; Harris and Contreras, 2014). When $\left[\mathrm{Ca}^{2+}\right]_{\mathrm{e}}$ falls, HCs will open as demonstrated in cardiomyocytes (Kondo et al., 2000), blood vessel endothelial cells (De Bock et al., 2012), blood cells (Eltzschig et al., 2006), and glial cells in brain slices (Torres et al., 2012). Almost all connexins display $\mathrm{HC}$ opening at low $\left[\mathrm{Ca}^{2+}\right]_{\mathrm{e}}$, including Cx26 and Cx30 (Lopez et al., 2013, 2016), Cx32 (GómezHernández et al., 2003), Cx37 (Puljung et al., 2004), Cx40 (Allen et al., 2011), Cx43 (Bruzzone et al., 2001; Contreras et al., 2003; Ye et al., 2003; Thimm et al., 2005), Cx46 (Paul et al., 1991; Ebihara et al., 2003), and Cx50 (Zampighi et al., 1999; Beahm and Hall, 2002). In general, $\mathrm{HCs}$ start to open when $\left[\mathrm{Ca}^{2+}\right]_{\mathrm{e}}$ decreases below $0.5 \mathrm{mM}$, but there are substantial differences between different connexins. In addition to $\mathrm{Ca}^{2+}, \mathrm{Mg}^{2+}$ also plays a role but $\mathrm{Ca}^{2+}$ exerts a dominant effect (Ebihara et al., 2003). Work from the Lal group with atomic force microscopy elegantly demonstrated that lowering $\left[\mathrm{Ca}^{2+}\right]_{\mathrm{e}}$ increases the $\mathrm{Cx} 43 \mathrm{HC}$ outer pore size (Thimm et al., 2005). In Cx32 (a liver, oligodendrocyte, and Schwann cell connexin), the high $\left[\mathrm{Ca}^{2+}\right]_{\mathrm{e}}$ closure has been traced down to Asp-169 and Asp-178 in the first half of EL2 that together form a ring of 12 Asp residues in the external vestibule of the HC (GómezHernández et al., 2003); this structure is conserved in $\mathrm{Cx} 30$ (an astrocyte and skin connexin), $\mathrm{Cx} 46$, and $\mathrm{Cx} 43$. For $\mathrm{Cx} 46$, the gate that closes the channel with millimolar $\left[\mathrm{Ca}^{2+}\right]_{\mathrm{e}}$ has been reported to be situated in extracellular direction relative to the position of Leu-35 in TM1 (Pfahnl and Dahl, 1999). Recent work from the Contreras group, based on computational molecular dynamics simulations of $\mathrm{Cx} 26$, has demonstrated that $\left[\mathrm{Ca}^{2+}\right]_{\mathrm{e}}$ closes the HCs by disrupting intersubunit salt bridges between Asp-50 and Lys-61 located in EL1 (Lopez et al., 2016). The authors proposed that $\mathrm{Ca}^{2+}$ binds to Asp-50 and Glu-47 in the same region, which would form a ring of 12 negatively charged residues as suggested for Cx32 (Gómez-Hernández et al., 2003). The Lopez et al. (2016) study also suggested that the actual gate closing the channel is presumably located deeper into the channel. The Barrio group brought up evidence that, for $\mathrm{Cx} 32, \mathrm{HC}$ opening triggered by lowering $\left[\mathrm{Ca}^{2+}\right]_{\mathrm{e}}$ involves transitions to a subconductance state $(\sim 18 \mathrm{pS})$ and from there to a main conductance state $(\sim 90 \mathrm{pS})$, with an inverse sequence occurring upon restoration of normal $\left[\mathrm{Ca}^{2+}\right]_{\mathrm{e}}$ (reviewed in Fasciani et al., 2013). Of note, $\left[\mathrm{Ca}^{2+}\right]_{\mathrm{e}}$ may also affect voltage gating as demonstrated for Cx46 (Ebihara and Steiner, 1993; Ebihara et al., 2003; Verselis and Srinivas, 2008). In particular, lowering $\left[\mathrm{Ca}^{2+}\right]_{\mathrm{e}}$ results in the unshielding of negative charges associated with the glycocalyx outside the cell, which attracts positive charge at the inside of the membrane capacitor and acts as a depolarizing stimulus on the voltage sensor. As a result, the voltage threshold for activation is lowered, resulting in a left shift of the voltage activation curve. Moreover, De Vuyst et al. (2006), reported that hemichannel-mediated ATP release triggered by exposure of $\mathrm{Cx} 32$ expressing cells to divalent-free solutions $\left(\mathrm{Ca}^{2 \pm}\right.$ - and $\mathrm{Mg}^{2+}$-free) was dependent on $\left[\mathrm{Ca}^{2+}\right]_{\mathrm{i}}$ changes, indicating that lowering $\left[\mathrm{Ca}^{2+}\right]_{\mathrm{e}}$ also influences HC opening by provoking $\left[\mathrm{Ca}^{2+}\right]_{\mathrm{i}}$ dynamics (De Vuyst et al., 2006).

5. $\mathrm{Ca}^{2+}$-Based Gating-Effects of Intracellular $\mathrm{Ca}^{2+}$. In contrast to $\left[\mathrm{Ca}^{2+}\right]_{\mathrm{e}}$ that only influences $\mathrm{HCs}$, alterations of $\left[\mathrm{Ca}^{2+}\right]_{i}$ affect both GJs and HCs. We first discuss effects on GJs and subsequently discuss more recently found effects of $\left[\mathrm{Ca}^{2+}\right]_{i}$ on $\mathrm{HCs}$.

a. Intracellular $\mathrm{Ca}^{2+}$ concentration effects on gap junctions. GJs close in response to large $\mathrm{V}_{\mathrm{j}}$ differences and acidification, but also in response to $\left[\mathrm{Ca}^{2+}\right]_{\mathrm{i}}$ elevation. Rose and Loewenstein (1975) demonstrated more than 40 years ago that injection of $\mathrm{Ca}^{2+}$ in one cell of a coupled cell-pair inhibits electrical coupling between cells (Rose and Loewenstein, 1975). The closing of GJs is considered to be protective, because it will isolate the cell displaying a pathologic process, thereby avoiding GJ-mediated bystander effects on healthy neighbors. The $\left[\mathrm{Ca}^{2+}\right]_{i}$ at which GJs close is rather variable between different studies and ranges from $300 \mathrm{nM}$ (Lazrak and Peracchia, 1993; Crow et al., 1994; Lurtz and Louis, 2007) to several micrometers (Rose and Loewenstein, 1975; Spray et al., 1982). This may result from differences between the involved connexins or differences between cell types; it may also be linked to 
the fact that global rather than microdomain $\left[\mathrm{Ca}^{2+}\right]_{\mathrm{i}}$ was measured. It furthermore appears that $\mathrm{Ca}^{2+}$ entry is more effective in closing GJs compared with $\mathrm{Ca}^{2+}$ store release (Lazrak et al., 1994). Dakin et al. (2005), making use of a photoactivated fluorescent probe, elegantly demonstrated that capacitative $\mathrm{Ca}^{2+}$ entry following store release was particularly potent in inhibiting junctional coupling (Dakin et al., 2005). Although $\mathrm{Ca}^{2+}$-inhibition of GJs has been reported to be fast (Lazrak et al., 1994), others have reported slower kinetics sometimes in the order of minutes (Churchill et al., 2001). Gap junction closure by $\left[\mathrm{Ca}^{2+}\right]_{\mathrm{i}}$ elevation is thought to be mediated by $\mathrm{Ca}^{2+}$-calmodulin signaling (reviewed in Peracchia, 2004; Lurtz and Louis, 2007). Cx32 has (at least) two calmodulin interaction sites located on the NT and CT (Torok et al., 1997), whereas Cx43 has one on its CL (Zhou et al., 2007) (Fig. 1). $\mathrm{Ca}^{2+}$ triggered GJ closure can be prevented by the calmodulin inhibitor W7 (Peracchia, 1987) and by a peptide that mimics the calmodulin interaction site on the CL of Cx43 (Zhou et al., 2007) (Lys-136-Ser-158 indicated as the "CaM" domain in Fig. 1). Peracchia and coworkers (Peracchia et al., 2000; Peracchia, 2004) suggested that $\mathrm{Ca}^{2+}$-calmodulin closure of $\mathrm{Cx} 32$ channels is mediated by a cork-plug model that involves channel pore obstruction by one of the calmodulin spherical lobes. Calmodulin may additionally act indirectly via calmodulin-dependent kinases below in section II.E.5.b). Finally, as remarked for $\mathrm{pH}$ effects, there are exceptional findings as well, in that $\left[\mathrm{Ca}^{2+}\right]_{\mathrm{i}}$ elevation or $\mathrm{Ca}^{2+}$-linked signaling has been reported to increase GJ coupling in heart as well as brain (Alev et al., 2008; De Pina-Benabou et al., 2001; Delage and Délèze, 1998; Siu et al., 2016). Clearly, $\left[\mathrm{Ca}^{2+}\right]_{\mathrm{i}}$ modulation of GJs may occur via multiple intermediate signaling steps, including various kinases; as a consequence, the kinetics can be slow and the outcome on channel function diverse.

b. Intracellular $\mathrm{Ca}^{2+}$ concentration effects on hemichannels. Although $\left[\mathrm{Ca}^{2+}\right]_{\mathrm{i}}$ elevation mostly closes GJs, its effects on HCs link to activation of channel opening. For Cx32, De Vuyst et al. (2006) reported, based on $\left[\mathrm{Ca}^{2+}\right]_{i}$ measurements and dye uptake or ATP release studies, that $\left[\mathrm{Ca}^{2+}\right]_{i}$ elevation triggers HC opening (De Vuyst et al., 2006); similar findings were reported in a follow up study performed on $\mathrm{Cx} 43$ (De Vuyst et al., 2009). In these studies, $\left[\mathrm{Ca}^{2+}\right]_{\mathrm{i}}$ was increased by a $\mathrm{Ca}^{2+}$ ionophore, triggering $\mathrm{Ca}^{2+}$ entry, or by photoactivation of caged (inactive) $\mathrm{IP}_{3}$ that triggers $\mathrm{ER} \mathrm{Ca}^{2+}$ release. The $\left[\mathrm{Ca}^{2+}\right]_{\mathrm{i}}$ response curve was peculiar in that elevations up to $500 \mathrm{nM}$ promoted $\mathrm{HC}$ opening, whereas $\left[\mathrm{Ca}^{2+}\right]_{\mathrm{i}}$ elevation above $500 \mathrm{nM}$ resulted in a gradual disappearance of the promotive effect, giving a convex-up bell-shaped response curve with a peak at $500 \mathrm{nM}$ and closure at $1 \mu \mathrm{M}\left[\mathrm{Ca}^{2+}\right]_{\mathrm{i}}(\mathrm{Fig}$. $2 \mathrm{~B})$. The closure at $1 \mu \mathrm{M}\left[\mathrm{Ca}^{2+}\right]_{\mathrm{i}}$ possibly acts as a brake to prevent excessive HC opening; a similar brakealbeit mediated by ATP-exists for Panx1 channels where ATP released via the channel inhibits the channel (Qiu and Dahl, 2009). The bell shape of HC responses to $\left[\mathrm{Ca}^{2+}\right]_{\mathrm{i}}$ elevation was also observed at the single channel level in HeLa cells overexpressing $\mathrm{Cx} 43$ (Wang et al., 2012a; Bol et al., 2016). In these cells $\left[\mathrm{Ca}^{2+}\right]_{\mathrm{i}}$ modulated the $\mathrm{HC}$ opening activity triggered by voltage steps to positive voltages ( $+40 \mathrm{mV}$ and higher) but it did not trigger $\mathrm{HC}$ opening by itself; imposing $\left[\mathrm{Ca}^{2+}\right]_{\mathrm{i}}$ elevations in the absence of the electrical trigger were ineffective in opening $\mathrm{HCs} .\left[\mathrm{Ca}^{2+}\right]_{\mathrm{i}}$ however had another interesting effect in that it lowered the voltage activation threshold for $\mathrm{HC}$ opening; even small $\left[\mathrm{Ca}^{2+}\right]_{\mathrm{i}}$ elevations from 50 to $200 \mathrm{nM}$ lowered the voltage threshold by $\sim 15 \mathrm{mV}$ (Wang et al., 2012a). Note that $\left[\mathrm{Ca}^{2+}\right]_{\mathrm{e}}$ also affects the voltage activation of $\mathrm{HC}$ opening but here the activation threshold is lowered by a decrease in $\left[\mathrm{Ca}^{2+}\right]_{\mathrm{e}}$ (Contreras et al., 2003). The HC enhancing effect of moderate $(\leq 500 \mathrm{nM})\left[\mathrm{Ca}^{2+}\right]_{\mathrm{i}}$ elevation has not only been observed in $\mathrm{Cx} 43$ expressing HeLa cells but has also been reported for acutely isolated ventricular cardiomyocytes (Wang et al., 2012a). Recent evidence obtained in mouse astrocytes furthermore demonstrates $\mathrm{HC}$ opening in response to $\left[\mathrm{Ca}^{2+}\right]_{\mathrm{i}}$ elevation with the membrane potential clamped at $-70 \mathrm{mV}$, i.e., without associated electrical stimulation (Meunier et al., 2017); similar observations are available for mouse and pig ventricular cardiomyocytes (unpublished data). The mechanism of the $\left[\mathrm{Ca}^{2+}\right]_{i}$ enhancing effect on $\mathrm{HC}$ opening activity has been linked to calmodulin, calmodulin-dependent kinase II, and several other factors; calmodulin-activation was particularly robust and was also effective when it was activated in $\mathrm{Ca}^{2+}$-independent manner by a $\mathrm{Ca}^{2+}$-like peptide called CALP (De Vuyst et al., 2009). The disappearance of enhanced $\mathrm{HC}$ function at higher, above $500 \mathrm{nM}\left[\mathrm{Ca}^{2+}\right]_{\mathrm{i}}$ elevation (Fig. 2B) was shown to depend on actomyosin contractility that acts to disrupt intramolecular/intersubunit CT-CL interaction (Ponsaerts et al., 2010; reviewed in Ponsaerts et al., 2012). In line with this, CT-CL disruption by high $(1 \mu \mathrm{M})\left[\mathrm{Ca}^{2+}\right]_{\mathrm{i}}$ was largely prevented by the myosin II ATPase inhibitor blebbistatin (Ponsaerts et al., 2008). The location of the actomyosin interaction site on the $\mathrm{Cx} 43 \mathrm{CT}$ is not known but the ZO-1 site has been excluded; involvement of the Drebrin site (see Fig. 1; Ambrosi et al., 2016), which like ZO-1 links to F-actin, still needs to be verified. Collectively, this work combined with the observations of Wang et al. (2013c), demonstrated that $\mathrm{CT}$ interaction with the $\mathrm{CL}$ is necessary for $\mathrm{Cx} 43 \mathrm{HCs}$ to become available for opening when a trigger is present; in the absence of CT-CL interaction, $\mathrm{HCs}$ are unresponsive to triggers and remain in the closed state (Fig. 2A) (Wang et al., 2013a,c). CT-CL interaction involves the CT9 domain (Fig. 1) that interacts with the CL-located L2 domain, whereby the negatively charged Asp-378 and Asp-379 residues on the CT play a crucial role (D'Hondt et al., 2013). 
Interestingly, supplying CT9 peptide prevented the HC closure at high $(1 \mu \mathrm{M})\left[\mathrm{Ca}^{2+}\right]_{\mathrm{i}}$ and thus removes the $\mathrm{HC}$ brake, most likely by directly binding to the L2 domain on the CL thereby acting as a CT substitute (Bol et al., 2016). Thus, in HCs, at least those composed of $\mathrm{Cx} 43$, CT-CL interaction is necessary for $\mathrm{HCs}$ to open, whereas the very same interaction closes the GJs, possibly by a particle-receptor scenario as explained previously (Fig. 2A). Data of Schalper et al. (2008) with fibroblast growth factor (FGF)-1 stimulation are in line with these observations; these authors reported that FGF-1 triggered $\mathrm{Cx} 43 \mathrm{HC}$ opening was dependent on $\left[\mathrm{Ca}^{2+}\right]_{\mathrm{i}}$ and lost upon $\mathrm{CT}$ truncation at position 257 (Schalper et al., 2008). It needs to be added that CT-truncated $\mathrm{Cx} 43$ (at Lys-258) still displays HC opening with low $\left[\mathrm{Ca}^{2+}\right]_{\mathrm{e}}$ stimulation (Kozoriz et al., 2010), indicating that the CT-CL interaction hypothesis might only be valid for triggers that come from the intracellular side such as $\mathrm{V}_{\mathrm{m}}$ steps to positive voltages or increases in $\left[\mathrm{Ca}^{2+}\right]_{\mathrm{i}}$. Of note, exposure of cells to low $\left[\mathrm{Ca}^{2+}\right]_{\mathrm{e}}$ may provoke $\left[\mathrm{Ca}^{2+}\right]_{\mathrm{i}}$ changes that act as the actual trigger of $\mathrm{HC}$ opening (De Vuyst et al., 2006). Besides effects of $\left[\mathrm{Ca}^{2+}\right]_{\mathrm{i}}$ elevation on $\mathrm{HC}$ gating, $\mathrm{Ca}^{2+}$ may influence connexin trafficking as well: $\mathrm{ER} \mathrm{Ca}^{2+}$ release, triggered by oxidative stress, promoted $\mathrm{Cx} 43$ $\mathrm{HC}$ opening by increasing the cell surface $\mathrm{HC}$ pool as determined from biotinylation experiments (Riquelme and Jiang, 2013).

Modulation of HCs and GJs in opposite directions, as observed in the context of CT-CL interaction (Fig. 2A), are not uncommon and have been reported for several conditions and signals. Also $\left[\mathrm{Ca}^{2+}\right]_{\mathrm{i}}$ distinctly influences the two channel types, because it promotes $\mathrm{HC}$ opening (at least when $\left[\mathrm{Ca}^{2+}\right]_{\mathrm{i}}$ is below $500 \mathrm{nM}$; see Fig. 2B) while it is well documented to inhibit GJs (exceptions exist here as well, as referred to earlier). Several other modulatory factors have opposite effects, most notably proinflammatory cytokines like tumor necrosis factor- $\alpha$ (TNF- $\alpha$ ) and interleukin 1- $\beta$ (IL1- $\beta$ ), or bacterial lipopolysaccharide (LPS), which all promote HC opening but close GJs (De Vuyst et al., 2007; Retamal et al., 2007a; Orellana et al., 2009); metabolic inhibition has a similar effect (Contreras et al., 2002). Along the same line, arachidonic acid inhibits GJs but promotes HC opening (Contreras et al., 2002; De Vuyst et al., 2007, 2009). By contrast, acidification inhibits both GJs and HCs (Spray et al., 1981; Trexler et al., 1999), a remarkable observation because low $\mathrm{pH}_{\mathrm{i}}$ closure of GJs is supposed to be mediated by CT-CL interaction while this very same interaction is expected to promote HC opening. However, Wang et al. (2012a) reported that $\mathrm{Cx} 43 \mathrm{HC}$ closure at low $\mathrm{pH}_{\mathrm{i}}$ disappears under conditions of strong cytoplasmic $\mathrm{Ca}^{2+}$ buffering with 1,2-bis(o-aminophenoxy)ethane- $\mathrm{N}, \mathrm{N}, \mathrm{N}^{\prime}, \mathrm{N}^{\prime}$-tetraacetic acid (commonly known as BAPTA), indicating that low $\mathrm{pH}_{\mathrm{i}}$ closure depends on $\left[\mathrm{Ca}^{2+}\right]_{\mathrm{i}}$ elevation (Wang et al., 2012a). Another possibility is that acidification has more direct effects on $\mathrm{Cx} 43$ that do not depend on CT-CL interaction.

6. Other Gating Effects. Several other HC opening triggers have been reported, but not all of them have been characterized at the electrophysiological level. Retamal et al. (2007b) reported that $\mathrm{Cx} 43 \mathrm{HCs}$ open in response to intracellularly applied reducing conditions making use of glutathione or dithiothreitol (Retamal et al., 2007b), an effect that was exercised at the level of channel gating. The latter experiments were done under normoxic conditions; under ischemic conditions (mimicked by metabolic inhibitors) astrocytic HCs also opened, but this was linked to an increase of the plasma membrane $\mathrm{HC}$ pool. The resulting increase of $\mathrm{HC}$ function was mediated by $S$-nitrosylation, possibly by NO acting at CT-located Cys residues, and inhibited by reducing agents (Retamal et al., 2006; reviewed in Sáez et al., 2005). Interestingly, $\mathrm{NO}$ is an $\mathrm{HC}$ permeant that may also activate HC opening (Retamal et al., 2006; Figueroa et al., 2013), as is the case for $\mathrm{Ca}^{2+}\left[\mathrm{Ca}^{2+}\right.$ permeates as well as activates channel opening by elevating $\left[\mathrm{Ca}^{2+}\right]_{i}$; NO can freely diffuse over the plasma membrane but when open HCs are available, they may facilitate NO diffusion (Figueroa et al., 2013)]. Work from the Lal group demonstrated Cx32 and $\mathrm{Cx} 43 \mathrm{HC}$ opening in response to $\mathrm{H}_{2} \mathrm{O}_{2}$-induced generation of reactive oxygen species (ROS) that appeared to involve $\mathrm{V}_{\mathrm{m}}$ changes (Ramachandran et al., 2007). Carbon monoxide (CO) inhibits $\mathrm{Cx} 43$ - and $\mathrm{Cx} 46$-based $\mathrm{HCs}$ independently of CT Cys residues, and the effect disappears by application of extracellularly acting reducing agents (León-Paravic et al., 2014). Effects of phosphorylation on gating of GJs/HCs are addressed section II.F.

\section{F. Connexin Posttranslational Modifications}

Many different connexin posttranslational modifications have been reported, including phosphorylation, ubiquitination, sumoylation, $S$-nitrosylation, palmitoylation, hydroxylation, acetylation, methylation, and $\gamma$-carboxyglutamation. However, the evidence supporting their prevalence and biologic significance varies considerably. Evidence that can support the existence and importance of posttranslation modifications includes detection of the modification using multiple analytical techniques, finding the modification in cells and tissues and observation of a biologic effect if that posttranslational modification is prevented. For example, detection of a posttranslational modification via direct incorporation of a radioactive element that is part of the posttranslational modification, detection via mass spectrometry, and/or detection via an antibody to the modification in connexin purified from cells would be convincing evidence that a posttranslational modification can occur. However, detection via a single method can be potentially misleading for several reasons. For example, amino acid modifications can be 
introduced during protein purification steps and then they are easily detected via high sensitivity mass spectrometers currently in use today. In vitro phosphorylation of a connexin, often just the $\mathrm{CT}$ region combined with a purified kinase, can indicate a phosphorylation event is possible, but without evidence that it happens in cells, the result by itself is not particularly important. Artificial introduction of a modification can also occur via overexpression of the substrate or the enzyme that catalyzes the modification in cells. Because all chemical reactions even within cells depend on the concentrations of the reactants, they may be possible under artificial conditions but not occur significantly in vivo. Therefore, multiple lines of evidence supporting the modification including its presence in vivo gives one more confidence that a modification was not artificially introduced. Furthermore, if modulation of the modification in vivo changes the biologic properties of GJ communication or other connexin characteristics (e.g., changes in interacting proteins or connexin localization) that would be good evidence of biologic relevance. For example, good evidence that a posttranslational modification is biologically meaningful might be derived from detection of the posttranslational modification by multiple methods in vivo (e.g., immunoprecipitation and detection via mass spectrometry or isotope incorporation and detection in cells via an antibody to the modification). Biologic relevance could be shown via modulation of the extent of modification through application of a targeted stimulus that elicits a physiologic change in connexin properties and elimination of the modulatory effect of the phosphorylation event when the specific connexin amino acid is mutated so it cannot be modified.

1. Phosphorylation. The best documented connexin posttranslational modification is phosphorylation. Connexin phosphorylation has been reported to affect connexin half-life, protein trafficking, incorporation into a GJ, GJ size, channel gating, and protein turnover (for recent reviews, see Pogoda et al., 2016; Solan and Lampe, 2016). Furthermore, wounding, ischemia, and other tissue insults have been shown to change connexin phosphorylation. Knock-in mice where three Ser to Ala or Ser to Glu point mutations were introduced in the gene for $\mathrm{Cx} 43$ at sites phosphorylated by casein kinase 1 (CK1) were highly susceptible or resistant to inducible arrhythmias, respectively (Remo et al., 2011). Knock-in mice expressing Cx43 with mitogen-activated protein kinase (MAPK) phosphorylation sites mutated to Ala showed reduced proliferation during arteriole injury and reduced neointima formation (Johnstone et al., 2012). Knock-in mice with $\mathrm{Cx} 43$ containing a Ser to Ala mutation at a protein kinase C (PKC) site were unresponsive to sphingosine-1-phosphate cardioprotective effects upon ischemia reperfusion injury (Morel et al., 2016). These results combined with those showing that these residues are phosphorylated in vivo essentially prove that connexin phosphorylation plays key roles in the physiologic response to injury.

Many connexins isolated from cellular preparations have been shown to incorporate radioactive phosphorous; demonstrate phosphatase-dependent shifts in mobility in SDS-PAGE; exhibit charge/mass ratios via mass spectrometry that are consistent with phosphorylation; and/or yield phospho-Ser, -Thr, and/or -Tyr in amino acid and peptide analyses. However, as for other posttranslational modifications, the extent of the evidence varies by connexin. There is at least one report of phosphorylation (discussed below) of most of the connexin $\alpha$ group members, including Cx37 (Morel et al., 2010), $\mathrm{Cx} 40, \mathrm{Cx} 43, \mathrm{Cx} 46$, and $\mathrm{Cx} 50$ and for $\mathrm{Cx} 36, \mathrm{Cx} 45$, and $\mathrm{Cx} 47$ (May et al., 2013), that belong to the $\gamma$ group ( $\alpha, \beta$ or $\gamma$ group assignations; see Cruciani and Mikalsen, 2006). Phosphorylation of other connexin group members, including Cx29 (Wiśniewski et al., 2010), Cx31 (Diestel et al., 2004), Cx31.9 (Nielsen and Kumar, 2003), and $\mathrm{Cx} 32$ (discussed below), has also been reported, but little biologic data supporting their biologic relevance exist. At least two connexin proteins (Cx26 and Cx33) have been reported to be not phosphorylated. In the next several paragraphs, we discuss the connexins for which significant data exist.

a. Cx43 phosphorylation. The most well-studied and characterized phosphoconnexin is $\mathrm{Cx} 43$. At least 19 of the 26 Ser and 4 of the 6 Tyr in the CT region of Cx43 have been identified as kinase substrates, and there has been some progress in the characterization of the network of kinases that phosphorylate $\mathrm{Cx} 43$ (phosphorylation sites are illustrated in Fig. 1 and major effects are summarized in Table 1). Of course, the level of evidence supporting these sites and the kinases involved varies considerably. Sites with high confidence include those phosphorylated by MAPK family members, PKC (especially $\delta$ and $\varepsilon$ ), Src, Akt, and CK1. MAPK3/MAPK1 (ERK1/2) are known to phosphorylate Ser-255, Ser-279, and Ser-282 in vitro (Warn-Cramer et al., 1996); these sites are phosphorylated in cells when MAPK is activated and GJ channel properties are modulated in a manner that is abrogated when the sites are mutated to Ala (Warn-Cramer et al., 1998). MAPK7 can also phosphorylate Ser-255 (Cameron et al., 2003), and Ser-262 has been identified as a substrate for CDK1 (Kanemitsu et al., 1998). Phosphospecific antibodies have been created for all of these sites and they react with $\mathrm{Cx} 43$ in Western blots and via immunofluorescence in the expected manner when MAPK is activated or inhibited. Although the exact sites and stoichiometry of the phosphorylation events are not clear, in vitro phosphorylation of $\mathrm{Cx} 43$ with purified CK1 yields phosphorylation at Ser-325, Ser-328, and Ser-330, and CK1 activation increases their phosphorylation, whereas inhibition decreases phosphorylation (Table 1). Assaying with a phosphospecific antibody for these sites reports decreased signal during hypoxia, and 
TABLE 1

Residues, kinases, and effects of $\mathrm{Cx} 43$ phosphorylation

\begin{tabular}{|c|c|c|c|}
\hline Assembly/Disassembly Residues & Kinases & Direction of Effect & References \\
\hline Ser-325,-328,-330 & CK1 & GJs $\uparrow$ & Cooper and Lampe (2002) \\
\hline Ser-364,-365 & & GJs $\uparrow$ & $\begin{array}{l}\text { TenBroek et al. (2001), Shah et al. (2002), Yogo et al. } \\
\text { (2002), Solan et al. (2007) }\end{array}$ \\
\hline \multirow[t]{2}{*}{ Ser-369, -373} & Akt/PKB & GJs $\uparrow$ & $\begin{array}{l}\text { Shah et al. (2002), Yogo et al. (2002), Park et al. (2007), } \\
\text { Dunn and Lampe, (2014) }\end{array}$ \\
\hline & & $\mathrm{HCs} \uparrow$ & Salas et al. (2015) \\
\hline Ser-372 & & GJs $\uparrow$ & $\begin{array}{l}\text { Sáez et al. (1997), Yogo et al. (2002), Park et al. (2007), } \\
\text { Dunn and Lampe (2014) }\end{array}$ \\
\hline Ser-368 & PKC & GJs $\downarrow, \uparrow$ & $\begin{array}{l}\text { Sáez et al. (1997), Axelsen et al. (2006), Procida et al. } \\
\text { (2009) }\end{array}$ \\
\hline Tyr-247, $-265,-313$ & Src, Tyk2 & GJs $\downarrow$ & $\begin{array}{l}\text { Lin et al. (2001), Ballif et al. (2008), Bonnette et al. } \\
\text { (2010), Solan and Lampe (2014), Li et al. (2016) }\end{array}$ \\
\hline \multicolumn{4}{|r|}{ 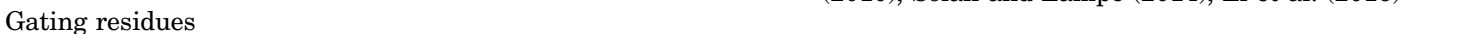 } \\
\hline Ser-255, -279, -282 & MAPK & GJs $\downarrow$ & Warn-Cramer et al. (1996) \\
\hline Ser-262 & p34cdc2, MAPK & GJs $\downarrow$ & $\begin{array}{l}\text { Kanemitsu et al. (1998), Lampe et al. (1998), Doble et al. } \\
\text { (2004) }\end{array}$ \\
\hline Ser-296, 297, 306 & & GJs $\uparrow$ & Axelsen et al. (2006), Procida et al. (2009) \\
\hline \multirow[t]{2}{*}{ Ser-368 } & $\mathrm{PKC}$ & GJs $\downarrow$ & $\begin{array}{l}\text { Sáez et al. (1997), Axelsen et al. (2006), Procida et al. } \\
\text { (2009) }\end{array}$ \\
\hline & & HCs $\downarrow$ & $\begin{array}{l}\text { Bao et al. (2004, 2007), De Vuyst et al. (2007), Hawat } \\
\text { and Baroudi (2008) }\end{array}$ \\
\hline
\end{tabular}

mutation of these sites affect the development of cardiac arrhythmia (Remo et al., 2011). Tyr-247 and Tyr-265 of Cx43 are known Src substrates and Tyr-301 and Tyr313 were identified as phosphorylated in general MS/MS-based screens for phosphotyrosine-containing proteins (Ballif et al., 2008; Bonnette et al., 2010). A recent report indicates that Tyk2 can phosphorylate the same Tyr residues as $\mathrm{Src}(\mathrm{Li}$ et al., 2016). The last 19 amino acids of $\mathrm{Cx} 43$ contain three sets of double Ser (Ser-364/Ser-365, Ser-368/Ser-369, Ser-372/Ser-373) that all have been reported to be phosphoacceptors (e.g., Yogo et al., 2002). Ser-368 is a very welldocumented substrate for classic PKCs, and early creation of a phosphospecific antibody for this site has led to dozens of reports of Ser-368 modulation in response to various cellular treatments and conditions. Akt phosphorylates Cx43 primarily at Ser-373 and secondarily at Ser-369 in vitro (Park et al., 2007), and the binding of a phosphospecific antibody for phosphorylated Ser-373 is decreased when Akt is inhibited in cells (Dunn and Lampe, 2014). Ser-364 (TenBroek et al., 2001) and Ser-365 (Solan et al., 2007) are also known to be phosphorylated particularly under conditions where PKA is activated, but the kinase(s) that actually phosphorylate these residues is less clear as it is a poor substrate for direct phosphorylation by PKA (TenBroek et al., 2001; Shah et al., 2002).

There are other documented $\mathrm{Cx} 43$ phosphorylation sites for which the kinase responsible is less clear. Ser-262 has been reported to be phosphorylated in response to PKC activation (Doble et al., 2004; Srisakuldee et al., 2014), but it was not a major substrate for purified classic PKCs (Sáez et al., 1997; Lampe et al., 2000), raising the question of whether PKC activation could cause activation of other kinases, potentially proline-directed kinases, because there is a Pro C terminus to Ser-262 fitting the consensus sequence and it is a known substrate for a prolinedirected kinase (Kanemitsu et al., 1998). A similar issue was observed for Src activation, which subsequently leads to MAPK and PKC activation and phosphorylation at Ser-255, Ser-262, Ser-279, Ser-282, Ser-368, Tyr247, and Tyr-265 (Solan and Lampe, 2008), potentially leading to confusion as to which sites and kinases actually are responsible for specific effects (Lin et al., 2001; Mitra et al., 2012). There are other reported sites for which we have less information, like Ser-296, Ser297, Ser-306, and Ser-314 (Procida et al., 2009), which need to be further investigated. Nonetheless, we know much more about $\mathrm{Cx} 43$ regulation via phosphorylation compared with the other connexins.

b. Cx32 phosphorylation. Cx32 was shown to be phosphorylated by metabolic labeling of hepatocytes, and phosphorylation levels were shown to increase with 8-bromo-cAMP (Sáez et al., 1986), forskolin, or phorbol ester treatment (Sáez et al., 1990). Ser-233 appeared to be a substrate for both PKA and PKC, and its phosphorylation level increased when the kinases were activated in cultured cells (Sáez et al., 1990). Epidermal growth factor receptor tyrosine kinase may also be involved in phosphorylation of $\mathrm{Cx} 32$ on tyrosine residues (Díez et al., 1998). However, the physiologic effects of all of the Cx32 phosphorylation events need to be studied further to better understand their biologic implications.

c. Cx36 phosphorylation. There has been a great deal of interest in the regulation of $\mathrm{Cx} 36$, because it has been found to be expressed in neurons as well as in dendrites of AII amacrine cells of the retina. Cx36 was shown to be a phosphoprotein by metabolic labeling and mass spectrometry (Urschel et al., 2006). Phosphospecific antibodies to PKA substrates Ser-110 and Ser-276 in Bass (Ser-293 in mammals) showed that large GJs in the inner plexiform layer showed higher levels of 
phosphorylation in dark-adapted and reduced levels in light-adapted retina (Kothmann et al., 2007). Dopamine-driven, PKA-dependent uncoupling of the AII amacrine cell network occurs via PKA activation of protein phosphatase $2 \mathrm{~A}$ and subsequent dephosphorylation of Cx36 (Kothmann et al., 2009).

d. Cx40 phosphorylation. $\mathrm{Cx} 40$ was shown to be phosphorylated via metabolic labeling (Traub et al., 1994). 8-Br-cAMP addition to Cx40-transfected cells resulted in a SDS-PAGE mobility shift in $\mathrm{Cx} 40$ and increased macroscopic GJ conductance between cell pairs (van Rijen et al., 2000). LPS or hypoxia and reoxygenation treatment of microvascular endothelial cells led to a reduction in $\mathrm{Cx} 40$ phosphorylation and electrical coupling between cells that was dependent on PKA (Bolon et al., 2008).

e. Cx45 phosphorylation. $\mathrm{Cx} 45$ has been convincingly shown to be phosphorylated via metabolic incorporation and phosphatase-dependent SDS-PAGE mobility shifts at primarily serine residues (Butterweck et al., 1994; Darrow et al., 1995), with less phosphotyrosine and phosphothreonine also being reported (Hertlein et al., 1998). Serines present within residues 374-393 were responsible for $89 \%$ of the phosphorylation and, in particular, Ser-381, -382, -384, and/or -385 were found to be important in regulating $\mathrm{Cx} 45$ stability, because mutation of different combinations of these reduced the half-life of the mutant version to $50 \%$ of wild-type Cx45 (Hertlein et al., 1998). Treatment of cells expressing $\mathrm{Cx} 45$ with 8-Br-cAMP or pervanadate increased $\mathrm{Cx} 45$ phosphorylation levels and decreased GJ conduction (van Veen et al., 2000).

f. Cx46 and Cx50 phosphorylation. These connexins are usually coexpressed in tissues, and several reports examined the phosphorylation status of both proteins so they are covered here together. Both migration shifts (Pelletier et al., 2015) and immunoprecipitation followed by mass spectrometry (Lin et al., 2004) have shown Cx46 and Cx50 to be phosphorylated at 9 and 18 serine residues, respectively (Wang and Schey, 2009). PKA phosphorylated Ser-395 of Cx50 and mutation of this residue to alanine attenuated increases in dye coupling and uptake caused by PLA activation (Liu et al., 2011). Phosphorylation of $\mathrm{Cx} 50$ can accelerate turnover (Yin et al., 2000) and susceptibility to caspase3-like protease cleavage (Yin et al., 2001).

2. Connexin Ubiquitination. Protein ubiquitination controls many aspects of cellular function by targeting substrates for degradation via the proteasome and through ubiquitin-dependent protein-protein interactions. There has been at least one report that $\mathrm{Cx} 26$ (Xiao et al., 2014), Cx32 (Kelly et al., 2007), and Cx40 (Gemel et al., 2014b) can be ubiquitinated, and there are many reports for $\mathrm{Cx} 43$. Furthermore, inhibition of the proteasome dramatically increases the levels of $\mathrm{Cx} 40$ (Gemel et al., 2014b) and Cx43 present within GJs (VanSlyke and Musil, 2005; Dunn and Lampe, 2014).
However, ubiquitin signaling is complex and there are many inconsistencies in the literature, potentially due to the variety of cellular systems under study that complicate our understanding of its role in connexin biology. For example, different reports indicate that Cx43 is mono-ubiquitinated (Leithe and Rivedal, 2004; Girão et al., 2009) or it is poly-ubiquitinated (Laing and Beyer, 1995; Laing et al., 1997; Ribeiro-Rodrigues et al., 2014; Martins-Marques et al., 2015b) and that it can preferentially be degraded by the proteasome or the lysosome. Given that the biologic consequences of monoversus poly-ubiquitination are potentially dramatically different (i.e., resulting in putative proteasomal degradation or activity regulation), these inconsistencies need some resolution. Most of these studies used cell lines where the ubiquitin is epitope or $6 \mathrm{xHis}$ tagged and overexpressed. Also, most used a $\mathrm{Cx} 43$ antibody to immunoprecipitate $\mathrm{Cx} 43$ and then cut the blot in two and probed the top half with antiubiquitin to show a ladder or smear of signal and the lower half with anti$\mathrm{Cx} 43$. The problem with this approach is that the levels and exposures of the two halves are not comparable and any protein that coprecipitates with $\mathrm{Cx} 43$ might be the actual ubiquitin acceptor rather than $\mathrm{Cx} 43$. In studies where estimates of the level of the putative ubiquitinated $\mathrm{Cx} 43$ isoform were made, it appeared to be much less than $1 \%$ of the total $\mathrm{Cx} 43$ comigrated with what might be an ubiquitinated isoform under conditions that should favor its accumulation (Girão et al., 2009; Chen et al., 2012b). Furthermore, conversion of all Lys in $\mathrm{Cx} 43$ to Arg to eliminate ubiquitination did not affect the accumulation of $\mathrm{Cx} 43$ in GJs in response to proteasomal inhibitors (Dunn et al., 2012) or during ER-localized degradation (Su et al., 2010). In fact, two other $\mathrm{Cx} 43$-interacting proteins that are ubiquitin substrates or ubiquitin associated, Akt (Dunn and Lampe, 2014) and CIP75 (Su et al., 2010), were found to regulate these processes in ubiquitin-dependent processes. Therefore, caution should be exercised when ubiquitin-related data are interpreted. On the other hand, a fusion protein of $\mathrm{Cx} 43$ and ubiquitin had a shorter half-life than wild-type $\mathrm{Cx} 43$ (Catarino et al., 2011). Clearly the ubiquitin system regulates connexin distribution. However, given its low levels and lack of a role in GJ size regulation and ER-associated degradation, further research needs to be performed to define the biologic function of $\mathrm{Cx} 43$ and connexin ubiquitination in general. One possible explanation consistent with at least some of these results is that ubiquitination of $\mathrm{Cx} 43$ does occur but only under specific situations such as autophagy (further discussed section II.F.4 below).

3. Other Potential Connexin Posttranslational Modifications. The isoelectric point of $\mathrm{Cx} 43$ is lower than predicted from its sequence in a manner thought to be independent of phosphorylation (Stockert et al., 1999), suggesting other modifications might be possible. 
A handful of reports indicate that connexins (e.g., $\mathrm{Cx} 26$ and $\mathrm{Cx} 43$ ) are also posttranslationally modified by $S$-nitrosylation (Retamal et al., 2006), SUMOylation (Kjenseth et al., 2012), acetylation (Colussi et al., 2011), palmitoylation, hydroxylation, methylation, and $\gamma$-carboxyglutamination. Methylation, acetylation, hydroxylation, palmitoylation, and $\gamma$-carboxyglutamination of $\mathrm{Cx} 26$ were reported as possible posttranslational modifications in an MS-based study of purified Cx26 (Locke et al., 2009). Although intriguing, as pointed out previously (Chen et al., 2013), this MS study used a limited search strategy based on accurate masses expected for $\mathrm{Cx} 26$ fragments rather than MS/MS analysis using broad search strategies, and this could have easily generated false discovery. Furthermore, the highly sensitive mass spectrometers in use today can generate false-positive posttranslational modification detection at many steps from protein purification (e.g., silver staining of gels) to MS data peak assignment (Larsen et al., 2006; Kim et al., 2016a). Therefore, confirmation via other techniques and studies will be necessary for all of these modifications, and if confirmed, understanding their biologic effect will become important.

4. Regulation of the Connexin Life Cycle via Posttranslational Modifications.

a. Connexin protein half-life. Although GJs appear as complex structures and can be difficult to disrupt during biochemical isolation, most connexin proteins have been shown to have short half-lives (1-5 hours), both in vivo and in cell culture (Crow et al., 1990; Musil et al., 1990; Laird et al., 1991; Lampe, 1994; Darrow et al., 1995; Beardslee et al., 1998; Hertlein et al., 1998; Lauf et al., 2002; Laird, 2006, 2010). However, the halflife of Cx30 (Kelly et al., 2015) and some isoforms of Cx50 (Jiang and Goodenough, 1998; Berthoud et al., 1999) appear to be much longer and more similar to typical integral membrane proteins (Chu and Doyle, 1985; Hare and Taylor, 1991). Why a cell would turnover connexin proteins so rapidly is not obvious. A short half-life with extensive regulation could allow the cell to control exquisitely processes dependent on connexin-binding protein interactions, $\mathrm{HC}$ function, or GJ communication. At least one region of $\mathrm{Cx} 43$ can control turnover, because it contains two putative Tyrbased sorting signals [Yxx $\phi$; where $\phi=$ hydrophobic (Fong et al., 2013)], including the key sequence $\mathrm{Y}^{286} \mathrm{KLV}$ (see Fig. 1), which upon Val to Asp mutation displays a threefold increased protein half-life (Thomas et al., 2003). As integral membrane proteins, connexins are synthesized in the ER but they lack a canonical membrane signal sequence and at least $\mathrm{Cx} 43$ does not oligomerize into a hexameric $\mathrm{HC}$ until reaching the trans-Golgi network (Musil and Goodenough, 1993). This delay in multimerization is hypothesized to provide a quality control step, because GJ assembly can be downregulated through endoplasmic reticulum-associated degradation during conditions of cellular stress (VanSlyke and Musil, 2002; Su et al., 2010).

b. Regulation of connexin export to the plasma membrane and gap junction assembly. Live cell and other imaging techniques show multiple regulatory aspects of $\mathrm{Cx} 26, \mathrm{Cx} 32$, and $\mathrm{Cx} 43$ transport to the plasma membrane, including microtubule-based vesicle transport (Martin et al., 2000; Johnson et al., 2002; Lauf et al., 2002; Shaw et al., 2007). Imaging studies have also shown that $\mathrm{Cx} 43$ can move from the plasma membrane into the periphery of a larger plaque; thus plaques grow by adding channels to the outside, and the oldest proteins are found in the center of the plaque where they get selectively turned over (Gaietta et al., 2002). Live cell imaging techniques also show $\mathrm{Cx} 43$ is highly motile with dynamic interactions with the cytoskeleton and events at GJs (e.g., Jordan et al., 1999, 2001; Martin et al., 2001; Lauf et al., 2002; Murray et al., 2004; Fiorini et al., 2008; Solan and Lampe, 2016). Phosphorylation of $\mathrm{Cx} 43$ can occur within 15 minutes of synthesis (Crow et al., 1990), and several kinases have been reported to regulate the assembly of GJs. When examined via Western immunoblot, many connexins demonstrate a phosphorylation-dependent reduction in SDS-PAGE mobility. $\mathrm{Cx} 43$ can often show three prominent bands sometimes labeled as P0, P1, and P2. Antibodies to Cx43 phosphorylated at Ser-365 show the P1 and P2 phosphoisoforms, which occur during the transition from the cytoplasm to the plasma membrane (Solan et al., 2007; Sosinsky et al., 2007). Furthermore, Ser-365 phosphorylation plays a "gatekeeper" role by preventing downregulation of GJ communication by subsequent Cx43 phosphorylation at Ser-368 (Solan et al., 2007). Several studies show that activation of cAMPdependent protein kinase (PKA) can stimulate $\mathrm{Cx} 43$ trafficking to the plasma membrane (Atkinson et al., 1995; Burghardt et al., 1995), resulting in enhanced GJ assembly (see Table 1) and increased phosphorylation at many of the last six serine residues in $\mathrm{Cx} 43$ (TenBroek et al., 2001; Yogo et al., 2002,2006; Solan et al., 2007). CK1 (Table 1) phosphorylates Cx43 on some combination of residues Ser-325, Ser-328, and/or Ser-330 during the transition of $\mathrm{Cx} 43$ from the plasma membrane into the GJ (Cooper and Lampe, 2002), and a phosphospecific antibody to this region recognizes only $\mathrm{Cx} 43$ present in the GJ and the $\mathrm{P} 2$ form of isoform $\mathrm{Cx} 43$ (Cooper and Lampe, 2002). Akt can phosphorylate Cx43 primarily at Ser-373 (see Table 1) and this step allows GJs to grow in size via a reduced interaction with $\mathrm{ZO}-1$ (Dunn and Lampe, 2014), which eliminates the welldocumented ability of ZO-1 to restrict GJ size (Hunter et al., 2005; Rhett et al., 2011). Although Cx43 GJ assembly is clearly regulated by a series of kinases that can fine tune GJ communication, our understanding of how GJ assembly is regulated for the other connexins is much less well understood. 
c. Regulation of gap junction turnover. Although we understand many aspects of GJ assembly, particularly for $\mathrm{Cx} 43$, and connexin protein turnover can easily be examined using pulse-chase methods, several aspects of GJ turnover remain unclear. For example, live cell imaging studies show that a single GJ can be relatively stable for hours but a neighboring one can be rapidly dispersed (e.g., Solan and Lampe, 2016). Proteasomal inhibitors can stabilize Cx43-containing GJs and make them larger, but they usually only have a mild effect on the total level of Cx43 (Lampe et al., 1998; Qin et al., 2003). However, lysosomal inhibitors can increase $\mathrm{Cx} 43$ protein levels, but the protein appears to build up in cytoplasmic membranes (Qin et al., 2003; Berthoud et al., 2004). Internalized annular junctions (connexosomes) (Severs et al., 1989; Jordan et al., 2001; Laird, 2006; Leithe et al., 2006; Piehl et al., 2007; Fong et al., 2012; Johnson et al., 2013; Nickel et al., 2013) are apparent in some cell types and in cells undergoing autophagy, where they colocalize with the clathrinadapter proteins disabled2 (Piehl et al., 2007), Atg14 and 9 (Bejarano et al., 2014), and the autophagosome membrane protein LC3 (Hesketh et al., 2010; Fong et al., 2012). Autophagy and degradation of multiple connexin isoforms have been linked (Iyyathurai et al., 2016). However, it is unclear whether this mechanism might account for most/all of GJ turnover or whether it occurs only in specialized circumstances such as nutrient deprivation. Part of the issue is that different treatments that affect clathrin-mediated and other internalization processes have only partial effects, making firm conclusions more difficult. Clearly more research is needed to understand whether multiple mechanisms for GJ turnover exist in vivo.

Although our knowledge of the mechanisms involved in GJ turnover is incomplete, we know that epidermal growth factor, 12- $O$-tetradecanoylphorbol acetate, Src activation, wounding, and extracellular ATP (Kanemitsu and Lau, 1993; Ruch et al., 2001; Schwiebert and Zsembery, 2003; Rivedal and Leithe, 2005; Chang et al., 2008; Dunn and Lampe, 2014) lead to $\mathrm{Cx} 43$ phosphorylation and loss of GJs. PKC (Lampe, 1994; Solan et al., 2003; Richards et al., 2004), MAPK (Johnstone et al., 2012), Src (Solan and Lampe, 2008), and Akt (Dunn et al., 2012) probably play at least some role in the regulation of $\mathrm{Cx} 43$ turnover. A kinase program that spatiotemporally activates $\mathrm{Cx} 43$ GJ turnover via sequential phosphorylation by Akt, MAPK, Src, and PKC in response to growth factors, wounding, and other stimuli has been proposed (Solan and Lampe, 2016). In this model, an Akt-mediated transient increase in GJ size depletes the nonjunctional $\mathrm{Cx} 43$ by rapid incorporation into a GJ, and the resulting larger GJ potentially reduces the energetics of annular junction formation by enabling membrane curvature during internalization. Src can clearly play a role, because its inhibition via PP2 treatment blocks growth factor-induced GJ turnover (Spinella et al., 2003; Gilleron et al., 2008). For many years it has been known that v-Src activity can downregulate GJ communication (Atkinson et al., 1981; Azarnia et al., 1988; Menko and Boettiger, 1988) coincident with an increase in tyrosine phosphorylation on $\mathrm{Cx} 43$ (Crow et al., 1990; Swenson et al., 1990). In LA25 cells that express active v-Src, Cx43 residues Tyr-247 (Src), Tyr-265 (Src), Ser-255 (MAPK), Ser-262 (MAPK), Ser-279/282 (MAPK), and Ser-368 (PKC) are all phosphorylated indicating co-activation of MAPK and PKC upon Src activation (Solan and Lampe, 2008). Phosphorylated Tyr-247 appeared to be preferentially present in larger GJ plaques (Solan and Lampe, 2014). Whether this distinct phosphoTyr-247 staining could potentially mark a portion of the GJ to facilitate interaction with components of the endocytic system is unknown. Src phosphorylation of $\mathrm{Cx} 43$ may trigger GJ endocytosis like it does with the NMDA receptor GluN3A (Chowdhury et al., 2013). There may also be three-way crosstalk regulation involved, because both $\mathrm{ZO}-1$ and Src can bind to the CT region of $\mathrm{Cx} 43$ and they can bind to each other as well (Sorgen et al., 2004; Gilleron et al., 2008; Kieken et al., 2009), whereas Akt phosphorylation at Ser-373 inhibits ZO-1 binding to $\mathrm{Cx} 43$ (Dunn and Lampe, 2014). Furthermore, the GJ blocker glycyrrhetinic acid causes GJs to adopt a looser packing arrangement (Goldberg et al., 1996) in a process that involves Src binding (Chung et al., 2007) and that leads to disruption of Cx43-ZO-1 interaction (Gilleron et al., 2008).

d. Phosphorylation effects on connexin channel gating. Phosphorylation effects on connexin channel function have been amply documented based on dye transfer studies making use of various channelpermeant fluorescent molecules. The data obtained with such approaches can be interpreted in several ways, because changes in dye transfer can be caused by alterations in the number of channels available, in the permeability properties of the channels, or in the channel gating characteristics. More fundamental insights into how phosphorylation alters GJ/HC function necessitates electrophysiological analysis at the single channel level, allowing the resolution of effects on open probability and single channel conductance, including main and residual conductance states. Only a few reports, reviewed in Moreno and Lau (2007), are available that have documented phosphorylation effects at a detailed unitary channel activity level. Kwak et al. (1995b) showed that cGMP-dependent Cx43 phosphorylation at Ser-259 (present in rat but not in human) reduced the GJ macroscopic conductance by decreasing the single channel main open state to a state with a lower conduction (Kwak et al., 1995b). Follow up work demonstrated distinct effects of phosphorylation by PKA, PKC, and PKG on different connexins (Cx26, $\mathrm{Cx} 43$, and $\mathrm{Cx} 45$ ) based on their biophysical single channel signature (Kwak et al., 1995a). Lampe et al. 
(2000) demonstrated that PKC phosphorylation at Ser-368 inhibits Cx43 GJs by shifting the unitary event activity in conductance histograms from $100 \mathrm{pS}$ centered events, corresponding to the main open state, to 50-60 pS events that correspond to a residual subconductance state; this work was based on comparing channel event activities in $\mathrm{Cx} 43$ wild type with those in a Cx43-S368A phosphodead mutant (Lampe et al., 2000). However, PKC has also been reported to increase the open probability of Cx43 GJs (Kwak et al., 1995c; Kwak and Jongsma, 1996), making the net effect on electrical current less clear. Bao et al. (2007) demonstrated that PKC phosphorylation, in addition to the effects on gating, also alters the size selectivity as suggested by sucrose permeation experiments (Bao et al., 2007).

For v-Src, Cottrell et al. (2003) reported that GJ inhibition was not mediated by alterations in the unitary conductances; this work was based on comparing event activities in $\mathrm{Cx} 43$ wild type with those in cells expressing $\mathrm{Cx} 43-\mathrm{Y} 247 \mathrm{~F}, \mathrm{Y} 265 \mathrm{~F}$ double mutant versions (Cottrell et al., 2003). Based on the fact that v-Src did not change the size of GJ plaques, suggesting that the number of GJ channels was not affected (Atkinson et al., 1986; Lin et al., 2001), the most probable effect of v-Src appeared to reside at the level of GJ channel open probability (Moreno and Lau, 2007). Further dye transfer studies by Cottrell et al. (2003) demonstrated that GJ channel permeability for Lucifer yellow and [2-(4-nitro2,1,3-benzoxadiazol-7-yl)aminoethyl]trimethylammonium was also affected by v-Src phosphorylation at Tyr-247 and Tyr-265; the effects were stronger at the level of dye transfer than at the level of current inhibition, which looks similar to the PKC effects. Interestingly, CT truncation at residue 245 , just two positions in NT direction relative to Tyr-247, removes GJ inhibition by v-Src (Zhou et al., 1999), making it possible that v-Src inhibition of GJs is the consequence of CT-CL particlereceptor (ball and chain) interaction (see section II.E.2); others have also linked v-Src to CT-CL interaction (Lau, 2005). Zhou et al. (1999) suggested that v-Src effects are indirect and mediated by MAPK effects at Ser-255, Ser-279, and Ser-282 (Warn-Cramer et al., 1998). In line with this, Cottrell et al. (2003) found that epidermal growth factor-activation of MAPK resulted in GJ inhibition that was not associated with alterations in the conductance states, as observed for v-Src; these authors furthermore demonstrated that MAPK-inhibition of GJs was absent in Cx43-S255A,279A,282A mutants. Of note, MAPK effects are fast (Kim et al., 1999) and have also been linked to CT-CL interaction (Harris, 2001).

Little is known about phosphorylation effects on the gating of channels composed of connexins other than Cx43. Sáez et al. (1986) reported that cAMP-stimulated phosphorylation of $\mathrm{Cx} 32$ induced a higher conductance state (Sáez et al., 1986). Similarly, van Rijen et al. (2000) reported that PKA activation, a well-known activator of GJ coupling downstream of cAMP, promoted $\mathrm{Cx} 40$ coupling by favoring a higher conductance state (van Rijen et al., 2000). Van Veen et al. (2000) demonstrated that pervanadate, which activates Tyr kinases, inhibited Cx45-based GJs by an effect that was concluded, based on exclusion of other possibilities, to decrease open probability (van Veen et al., 2000).

The same paucity of data applies to effects of phosphorylation at the level of gating of HCs. Several papers have suggested that MAPK family members may promote Cx43 HC opening (Schalper et al., 2008; De Vuyst et al., 2009; Avendano et al., 2015), but others have reported opposite effects (Kim et al., 1999; Riquelme et al., 2015). These results were based on indirect measures of HC function, mostly dye uptake or ATP release assays. Dye uptake studies also demonstrated that Akt/PKB, activated by metabolic inhibition, increased $\mathrm{Cx} 43 \mathrm{HC}$ function by increasing the $\mathrm{HC}$ pool in the plasma membrane as judged from biotinylation experiments (Salas et al., 2015). For PKC, all available data seem to point to inhibition of $\mathrm{HC}$ activity (Bao et al., 2004, 2007; De Vuyst et al., 2007); Hawat and Baroudi (2008) confirmed this at the electrophysiological macroscopic conductance level and further demonstrated that $\mathrm{PKC}_{\varepsilon}$ had more potent effects than $\mathrm{PKC}_{\beta}$ or $\mathrm{PKC}_{\delta}$ (Hawat and Baroudi, 2008). Clearly, more work is necessary here to resolve $\mathrm{HC}$ modulation by various kinases at the unitary current level and to determine how it affects gating and whether this relates to CT- or NT-linked conformational changes. Table 1 summarizes the effects of various kinases and target residues.

\section{Pharmacological Modulation of Connexin Channels}

The pharmacology of connexin channels is complex. First of all, specific inhibitors like those available for $\mathrm{Na}^{+}, \mathrm{Ca}^{2+}$, and $\mathrm{K}^{+}$channels are not available. Second, most substances inhibit both GJs and HCs, except for some substances that are discussed below. Third, almost all inhibitors act in the micromolar range, not in the nanomolar range. Pharmacological modulation of GJs/HCs can, in principle, be achieved in various ways acting at distinct levels, including 1) connexin biosynthesis/expression, 2) connexin trafficking and $\mathrm{HC}$ assembly, 3) docking of HCs and formation of GJs, 4) formation of GJ plaques, 5) GJ disassembly by internalization and degradation, 6) the number of channels (by mechanisms related to 1 to 5), 7) channel gating, and 8) pore block or alterations in channel permeability when pore block is incomplete. Second messengers, posttranslational modifications, ions like $\mathrm{Ca}^{2+}, \mathrm{H}^{+}$, and membrane potential all act at least at one of these various levels, as described in the preceding sections. Several pharmacological agents may act at these different levels, either directly or indirectly by influencing intracellular signaling cascades (Table 2; 
reviewed in Dhein, 2004; Salameh and Dhein, 2005). For example, the fatty acid oleic acid inhibits GJ channels via PKC phosphorylation of Ser-368 (Huang et al., 2004). Lindane, a carcinogenic insecticide, inhibits GJs by MAPK activation that promotes the displacement of membrane-located $\mathrm{Cx} 43$ to the intracellular compartment (Mograbi et al., 2003). Tedisamil, a class III antiarrhythmic agent, promotes GJ conductance by acting on PKA (De Mello and Thormahlen, 1999).

\section{A. Connexin Channel Inhibitors}

Several chemical classes of connexin channel inhibiting substances exist (reviewed in Salameh and Dhein, 2005; Bodendiek and Raman, 2010; Verselis and Srinivas, 2013). The best known and widely used substance in experimental work is carbenoxolone, a derivative of the licorice-derived glycyrrhetinic acid, which has better water solubility compared with $18-\alpha$-glycyrrhetinic acid and 18- $\beta$-glycyrrhetinic acid; carbenoxolone inhibits GJs with an $\mathrm{IC}_{50}$ in the range of $\sim 50 \mu \mathrm{M}$ (see Table 1 in Spray et al., 2006). The mechanism of action of this family of licorice-based molecules on connexin channels is poorly defined. 18- $\alpha$ Glycyrrhetinic acid has been reported to inhibit connexin synthesis (by decreasing transcription) and/or to promote connexin turnover at concentrations above $20 \mu \mathrm{M}$ (Guo et al., 1999), whereas $18-\beta$-glycyrrhetinic acid has been demonstrated to dephosphorylate $\mathrm{Cx} 43$ by type 1 or type 2 A protein phosphatases (Guan et al., 1996). For carbenoxolone, several authors reported reduced Cx43 expression after prolonged (6-24 hours) carbenoxolone exposure (Herrero-González et al., 2009; Wang et al., 2009; Yulyana et al., 2013; Kim et al., 2017). Goldberg et al. (1996) reported no changes in connexin expression or location but alterations of $\mathrm{HC}$ packing in GJ plaques (Goldberg et al., 1996). Carbenoxolone is not a specific connexin channel blocker; for example, it is a commonly used drug in the treatment of gastrointestinal ulceration in Asian countries. Because of its steroid hormone backbone structure, it has intrinsic mineralocorticoid effects. Additionally, it has several other side effects (reviewed in Connors, 2012): it inhibits 11- $\beta$ hydroxysteroid dehydrogenase and thereby influences glucocorticoid synthesis (Monder et al., 1989; Sandeep et al., 2004), inhibits voltage-gated $\mathrm{Ca}^{2+}$ currents $\left[\mathrm{IC}_{50} \sim 50 \mu \mathrm{M}\right.$ (Vessey et al., 2004)], has direct effects on GABA receptors (Connors, 2012), inhibits synaptic currents and action potentials (Rekling et al., 2000; Rouach et al., 2003; Tovar et al., 2009; Beaumont and Maccaferri, 2011), and acts as an anti-inflammatory agent (Amagaya et al., 1984; Inoue et al., 1989), which may all contribute to the observed effects beyond the effect on connexin channels as illustrated in, e.g., cardiac ischemia models (Haleagrahara et al., 2011). Carbenoxolone also inhibits pannexin channels with an $\mathrm{IC}_{50} \sim 5 \mu \mathrm{M}$ (Bruzzone et al., 2005), whereas higher concentrations $(\sim 50 \mu \mathrm{M}$ or higher) are necessary to inhibit GJs (see Table 1 in Spray et al., 2006); of note, connexin HCs are also inhibited by concentrations in the $\mathrm{IC}_{50}$ range of $5 \mu \mathrm{M}$. Also $\mathrm{P}_{2} \mathrm{X}_{7}$ receptors are inhibited by carbenoxolone with an $\mathrm{IC}_{50}$ in the range of $175 \mathrm{nM}$ (Suadicani et al., 2006).

Other nonspecific connexin channel inhibitors include long-chain alcohols such as heptanol and octanol, which incorporate in the plasma membrane and act by altering membrane fluidity that somehow affects the channel (Bastiaanse et al., 1993). Another lipophilic agent is halothane, an inhalational general anesthetic that inhibits GJs at supra-anesthetic concentrations (Wentlandt et al., 2006)) in the order of $2 \mathrm{mM}$ (Burt and Spray, 1989); it inhibits GJs by decreasing the open probability (He and Burt, 2000). Fatty acids like oleic acid, a mono-unsaturated fatty acid present in olive oil with various biologic effects, inhibits GJs indirectly via PKC phosphorylation, as mentioned above. Fatty acid amides like anandamide, an endogenous cannabinoid receptor agonist, also blocks GJs (Venance et al., 1995). The fatty acid arachidonic acid, a poly-unsaturated fatty acid in plasma membrane phospholipids involved in inflammatory signaling, takes a special place, because it inhibits GJs but promotes $\mathrm{HC}$ opening (Contreras et al., 2002; De Vuyst et al., 2007,2009). Its $K_{\mathrm{D}}$ for GJ inhibition is $\sim 4 \mu \mathrm{M}$, and inhibition has been linked to decreased open probability (Schmilinsky-Fluri et al., 1997).

Fenamates like flufenamic acid, niflumic acid, and meclofenamic acid are nonsteroidal anti-inflammatory molecules that inhibit cyclo-oxygenase. They inhibit GJs with an $\mathrm{IC}_{50}$ in the order of $25-40 \mu \mathrm{M}$ (Harks et al., 2001; Srinivas and Spray, 2003) and have been also demonstrated to block HCs (Gomes et al., 2005). Flufenamic acid acts by decreasing the open probability (Srinivas and Spray, 2003). Quinine and mefloquine are antimalarial drugs that display some specificity for inhibiting $\mathrm{Cx} 36$ and $\mathrm{Cx} 50$; mefloquine displays an $\mathrm{IC}_{50}$ of $300 \mathrm{nM}$ and $\sim 1 \mu \mathrm{M}$ for $\mathrm{Cx} 36$ and $\mathrm{Cx} 50$ (Cruikshank et al., 2004), i.e., very low concentrations compared with other connexin channel inhibitors, making it the most potent GJ inhibitor currently known. However, the downside is that the substance also has multiple side effects (see Verselis and Srinivas, 2013). Quinine has been shown to promote HC opening (Stout et al., 2002), similar to arachidonic acid. Derivatives of the triarylmethane cotrimoxazole, an antibiotic, also has connexin specificity targeted to $\mathrm{Cx} 50$; it acts with an $\mathrm{IC}_{50}$ of 1-2 $\mu \mathrm{M}$ and has a 10 -fold higher specificity for Cx50 compared with other connexins as well as $\mathrm{Na}^{+}$and $\mathrm{K}^{+}$channels (Bodendiek et al., 2012; reviewed in Verselis and Srinivas, 2013).

Several other connexin channel-inhibiting compounds are best known for their other actions, including 2-amino ethoxydiphenyl borate, a blocker of $\mathrm{IP}_{3}$ receptors and store-operated $\mathrm{Ca}^{2+}$ entry; polyamines like 


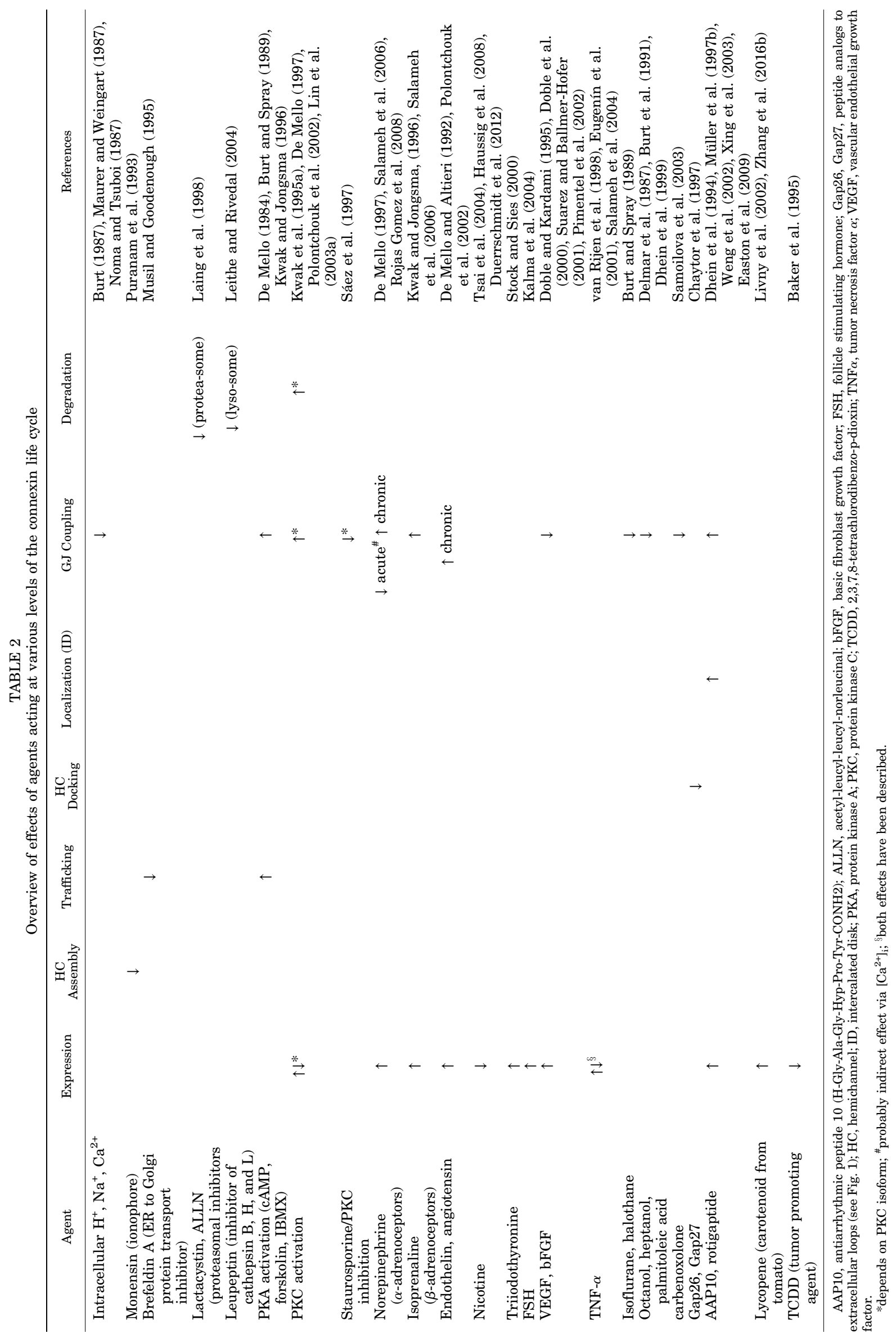


spermine and spermidine acting on NMDA channels; and several other targets, 5-nitro-2-(3-phenylpropylamino)benzoic acid, a chloride channel blocker; disodium 4,4'-diisothio cyanatostilbene-2,2'-disulfonate, an anion transport blocker; and certain triphenylmethanes, triphenylethanes, triarylmethanes, and cyclodextrins (Bodendiek and Raman, 2010).

\section{B. Peptide Modulators of Connexin Channels}

Improved specificity has been sought by making use of peptides that interact, directly or indirectly, with the connexin protein and thereby modulate, inhibit, or promote its channel function. Most of the peptides used are identical to a sequence on the connexin protein, e.g., Gap26 and Gap27, whereas others like AAP10 are unrelated to the connexin protein.

1. Mimetic Peptides of the Extracellular Loops.

a. GAP26 and GAP27 peptides. Warner et al. (1995) were the first to report that peptides identical to sequences on the connexin EL domains (called "connexin mimetic peptides") inhibited the synchrony of chick myoball contractions used as an indirect assay of GJ coupling (Warner et al., 1995). The starting point for this work was the hypothesis that supplying exogenous peptides identical to crucial domains on the ELs would interact with the ELs and thereby interfere with or prevent the docking of opposed HCs. By using their myoball approach, they identified several conserved motifs in the ELs that inhibited GJs when applied as synthetic peptides. For EL1, the crucial conserved connexin motifs were QPG and SHVR, whereas the conserved SRPTEK motif was crucial for EL2 (Warner et al., 1995). Peptides containing these motifs were, respectively, dubbed Gap26 (EL1) and Gap27 (EL2). For Gap26, the crucial motifs rather look like VCYD and FPISH for various connexins (e.g., Cx26, Cx32, Cx37, $\mathrm{Cx} 40$, and $\mathrm{Cx} 43$ and others) instead of QPG and SHVR (VCYD and FPISH motifs are illustrated in Fig. 1). The ability of SRPTEK-containing peptides to inhibit GJ coupling was enhanced by adding several amino acids from the putative membrane-spanning region. For example, Gap27 (Cx43 sequence SRPTEKTIFII in human and mouse) contains three amino acids that dig into the plasma membrane at TM4 (Fig. 1). Inhibition of GJs by Gap26/Gap27 is characterized by an $\mathrm{IC}_{50}$ of $20-30 \mu \mathrm{M}$ (Chaytor et al., 1997) and the peptides need to be applied at $200-300 \mu \mathrm{M}$ to obtain maximal effects. However, these data were not obtained directly by measuring GJ coupling but by indirect measurements consisting of phenylephrine-induced rhythmic activity of endothelium-denuded rabbit superior mesenteric artery rings. GJ block by Gap26/27 is never complete [ $\sim 95 \%$ complete for Gap26 (Desplantez et al., 2012b)]. Although Gap26 and Gap27 contain the conserved sequences VCYDXXFPISH and SRPTEK, respectively, they also contain amino acids that are less conserved. For example, the Gap27 sequence for $\mathrm{Cx} 43$ is
SRPTEKTIFII (see Fig. 1; further referred to as ${ }^{43} \mathrm{Gap} 27$ ), which is the same sequence as for $\mathrm{Cx} 37$ (hence its denomination as ${ }^{37,43} \mathrm{Gap} 27$ ) but slightly different for Cx40: SRPTEKNVFIV $\left({ }^{40} \mathrm{Gap} 27\right)$. By contrast, the Gap26 sequence for $\mathrm{Cx} 37$ and $\mathrm{Cx} 40$ is the same: VCYDQAFPISHIR $\left({ }^{37,40} \mathrm{Gap} 26\right)$ but different for Cx43 (VCYDKSFPISHVR, Fig. 1). Experimental work with these peptides (or close derivatives) on vascular cells has demonstrated distinctive effects on $\mathrm{Cx} 37$, Cx40, and Cx43 (Kwak and Jongsma, 1999; Ujiie et al., 2003; Martin et al., 2005; Young et al., 2008; Billaud et al., 2011), suggesting they display some isoform, specificity.

The exact way of how Gap26 and Gap27 inhibit GJs is currently not known but several hypotheses exist (Evans and Boitano, 2001). First, the peptides may interact with the ELs of HCs, thereby preventing the interaction and docking of two complementary HCs to form a new GJ channel (Leybaert et al., 2003). As a result, fewer HCs will be incorporated into GJs (see section II.B). Because the connexin half-life is $1-5$ hours, GJ inhibition would in this case be expected to occur on a similar time scale as hindered docking would decrease the number of GJ channels as a result of the ongoing GJ disassembly process. The kinetics of Gap26/Gap27 inhibition of Cx43-based GJs has an estimated half-time of $\sim 13$ minutes based on electrophysiological measurements (Desplantez et al., 2012b). Indirect measurements based on $\mathrm{Ca}^{2+}$ waves indicate a half-time in the order of 15-30 minutes for Gap26 and 30-60 minutes for Gap27 (Boitano and Evans, 2000). By contrast, exposure times as long as 6 hours have been reported to have no effect on FRAP-based dye coupling (Decrock et al., 2009a) and 24 hours is necessary to see an effect on coupling (De Bock et al., 2011). At the other extreme are observations based on capacitance measurements that indicate GJ inhibition within 5 minutes (Matchkov et al., 2006). Possibly, capacitance measurements may be influenced by effects on $\mathrm{HCs}$, because those channels are typically blocked on a time scale of a few minutes (Desplantez et al., 2012b; Wang et al., 2012a) (HC effects are discussed below). It is currently not known how Gap26/27 interact with the ELs; there are no reports on direct molecular interactions based on surface plasmon resonance or NMR studies. Work with atomic force microscopy whereby Gap26 was covalently linked to the scanning tip has demonstrated interaction with the Cx43 ELs based on the unbinding force experienced by retracting the scanning tip after interaction was established (Liu et al., 2006). As pointed out earlier (see section II.B.2), HC docking involves EL1-EL1 and EL2-EL2 interactions between opposed connexins, but EL1-EL2 and EL2-EL1 interactions are also possible. Recently, the Green group performed competition experiments with Peptide5, a connexin HC-inhibiting peptide based on the EL2 SRPTEK (“Gap27”) motif (Fig. 1), with various neighboring 
EL2-based sequences (Kim et al., 2017). They found that Peptide5 inhibition was counteracted by equimolar addition of an EL2 peptide with a sequence N-terminally adjacent to the Peptide5 sequence, indicating that Peptide5 interacts with EL2. Most interestingly, a peptide composed of the entire EL1 sequence acted synergistically with Peptide5, giving significantly increased inhibition. Thus, EL2-derived peptides appear to interact with EL2 and not with EL1; it remains to be determined whether EL1-based peptides interact with EL1. Additionally, combination of EL1- and EL2-based peptides may provide stronger inhibition.

A second possibility for Gap26/27 effects may relate to direct interactions of these peptides with existing GJs, resulting either in the separation of $\mathrm{HCs}$ from existing GJs or in effects on GJ gating. Separation of docked HCs is in principle possible, and continuation of this process would result in unzippering of the GJ followed by internalization and breakdown. Solan and Lampe (2014) have suggested that the switch between unzippering and uptake of the complete GJ as an annular junction (see section II.B.4) is determined by distinct Cx43 phosphorylation patterns (Solan and Lampe, 2014). Although it is conceivable that Gap peptides may have access to the intercellular space and HC docking region via the edge of the junctional plaque, there is currently very little evidence for the unzippering scenario given the tenacity of adhesion of docked HCs. The other possibility is that Gap26/27 interaction with GJs results in effects on GJ channel gating properties. Such effect has been described for an EL2 peptide called P180-195 composed of amino acids Ser-180-Gln-195 of Cx43 (Fig. 1), which appeared to decrease the dwelling in the subconductance (residual) state without pronounced alterations to the main conductance state (Kwak and Jongsma, 1999). However, peptide exposure times were very long (overnight) in this study. In the absence of any additional evidence, the possibility of direct actions of the Gap peptides on the gating or unzippering of GJs remains open for further investigations.

Thus far, most evidence points to interaction of Gap26/27 peptides with HCs not incorporated in GJs (see Fig. 3 in Leybaert et al., 2003). Most notably, Gap26/27 peptides inhibit HCs faster than GJs: macroscopic current measurements have suggested HC inhibition within 5 minutes (Desplantez et al., 2012b) and analysis at the unitary current level has demonstrated a half-time of $\sim 100$ seconds (time constant $\tau$ of 148 seconds) and $\sim 150$ seconds ( $\tau=223$ seconds) for Gap26 and Gap27, respectively (Wang et al., 2012a). Removal of inhibition occurs within a time frame of 2.6 minutes (Desplantez et al., 2012b). As discussed earlier, GJ inhibition takes tens of minutes to hours, indicating that the peptides first interact with $\mathrm{HCs}$ and subsequently prevent the docking process, thereby inhibiting GJs. Although HC block occurs within minutes, this is still slow in terms of action dynamics on a channel protein. One reason could be that the interaction site on the ELs is not immediately accessible and needs some molecular rearrangements or unfolding. Another possibility is that there are other interactions with connexin domains that are buried deeper into the channel pore or that are only accessible when the channel is open; the latter option still needs to be tested by evaluating the use dependence of block. In fact, the time-dependence curve of $\mathrm{HC}$ block in Wang et al. (2012a) may contain some use-dependent effect, because the protocol of this experiment involved repetitive application of $\mathrm{HC}$ opening voltage steps. In terms of mechanisms of $\mathrm{HC}$ inhibition, the classic possibilities are decreased number of channels, pore block, decreased unitary conductance, or effects on gating (reviewed in Wang et al., 2013a). Effects on the number of channels and single channel conductance have been excluded (Wang et al., 2012a), at least for an exposure time of 30 minutes. Pore block occurs at high concentrations, in the order of $1 \mathrm{mM}$, in which case control peptides (e.g., with a scrambled sequence) as well as active peptide inhibit HCs (Wang et al., 2012a), demonstrating that block becomes unspecific at these high concentrations. The most probable effect of Gap26/27 resides at the level of channel gating: Wang et al. (2012a) reported that Gap26/27 decreases the dwelling in the main conductance state without affecting the subconductance state (Wang et al., 2013a); these findings are opposite of the effects reported by Kwak and Jongsma (1999) with the P180-195 EL2 peptide (Fig. 1), which demonstrated decreased dwelling in the subconductance state without changes for the main conductance state. As transitions from fully closed to the main open state involve the slow loop gate, it can be concluded that Gap26/27 inhibition results from closure of the loop gate. Gap26/27 also have other effects on gating, in that they increase the $V_{m}$ threshold for voltage activation. Although substantial mechanistic insights have been obtained on mechanisms of Gap26/27 inhibition of electrically triggered $\mathrm{HC}$ opening, little experimental evidence is available on their effect on gating mechanisms involved in chemically triggered HC opening. However, as the slow loop gate plays a central role in chemical gating, it looks obvious to suppose that Gap26/27 would also inhibit loop gate opening triggered by, e.g., $\left[\mathrm{Ca}^{2+}\right]_{\mathrm{i}}$ elevation or exposure to proinflammatory or ischemic conditions. Just like is the case for GJs, Gap26/27 block of HCs is incomplete. Gap26 HC block appears to be less complete ( $\sim 65 \%$ inhibition) than observed with Gap27 ( $~ 84 \%$ inhibition; at supramaximal concentrations). On the other hand, the $\mathrm{IC}_{50}$ of Gap26 is lower $(\sim 80 \mu \mathrm{M})$ than for Gap27 $(\sim 160 \mu \mathrm{M})$ (Wang et al., 2012a). Note that the $\mathrm{IC}_{50}$ of these peptides for HC inhibition are higher than those reported by Chaytor et al. (1997) for inhibition of GJs; however, as 
mentioned earlier, the assay in the Chaytor et al. (1997) study was a very indirect one based on rhythmicity of blood vessel rings exposed to phenylephrine, whereas the HC assays of Wang et al. (2012a) were based on measurements of unitary $\mathrm{HC}$ opening activities. Although Gap26/27 peptides have some specificity for different connexin isoforms, their selectivity has been challenged, because they were found also to inhibit Panx1 channels (Dahl, 2007; Wang et al., 2007a). Limited effects of Gap26/27 peptides on other proteins like Panx 1 channels are not unexpected, given the high concentration of $200 \mu \mathrm{M}$ or higher needed to block connexin channels; however, the effect of Gap26/27 on connexin channels is much stronger than on Panx1 channels. It is therefore advised to use concentrations less than $200 \mu \mathrm{M}$, because nonspecific effects will progressively appear; at $1 \mathrm{mM}$, nonspecific block is maximal with no difference between active and scrambled peptide sequences (Wang et al., 2012a).

b. Peptide5. Peptide5 is a peptide based on the SRPTEK domain with sequence VDCFLSRPTEKT (Fig. 1) first reported by O'Carroll et al. (2008). This peptide came out as most potent after a screen of several peptides mimicking sequences on the $\mathrm{Cx} 43 \mathrm{ELs}$ (including some containing TM portions) for their potential to inhibit cell swelling, astrogliosis, and neuronal cell death in an in vitro model of spinal cord injury. They found that Peptide5 inhibited HCs at concentrations of $5 \mu \mathrm{M}$, whereas hundreds of micromolars were necessary to inhibit GJs. Follow up work demonstrated significant neuroprotective and inflammation dampening effects of this peptide in various models including brain ischemia in fetal sheep (Davidson et al., 2012b, 2014), retinal ischemia (Danesh-Meyer et al., 2012), and spinal cord injury (O'Carroll et al., 2013) and subsequently extensively tested for its neuroprotective potential (see section VIII). Recent work from Kim et al. (2017) demonstrated that HC block is very sensitive to alterations of the Peptide5 sequence (either single amino acid substitutions for Ala or truncations at the NT or CT side) and that the SRPTEKT sequence was not sufficient on its own to block HCs (Kim et al., 2017). By contrast, GJ block was not significantly altered by these modifications and SRPTEKT acted equally well as Peptide5. This suggests distinct interactions sites for Peptide5 inhibition of HCs and GJs. Most interestingly, combining Peptide5 with EL1 peptide (entire loop sequence) increased the potency of HC inhibition. Given the fact that HC inhibition (but not GJ inhibition) is very sensitive to sequence alterations of Peptide5, including single amino acid substitutions outside the SRPTEKT sequence, suggests a possible sequence specificity for HC inhibition, an option that needs to be further tested. Peptide5 inhibition of GJs appeared to be mediated by altered distribution of $\mathrm{Cx} 43$ without influencing the expression level (Kim et al., 2017). Currently there are no data available on Peptide5 effects at single channel resolution to judge its effect on unitary conductance and gating.

\section{Mimetic Peptides of Intracellular Connexin} Sequences.

a. GAP19 and L2-specific hemichannel blocking peptides. Although Peptide5 inhibits HCs only at low concentrations and gives combined GJ/HC inhibition at high concentrations, Gap19 and L2 peptides inhibit HCs while they prevent the closure of GJs, i.e., they have opposite effects on GJs and HCs (Fig. 2A). Gap19 is a peptide mimicking a nine amino acid sequence on the CL of $\mathrm{Cx} 43$ located within the L2 sequence (Fig. 1). Combined work of the Bultynck and Leybaert groups has demonstrated that L2 peptide, which was known to prevent GJ closure upon acidification (see section II.E.2) unexpectedly inhibited Cx43 HCs (Ponsaerts et al., 2010; reviewed in Iyyathurai et al., 2013). They further identified Gap19 peptide as a sequence within the L2 domain that is flanked at both sides by $\alpha$-helices at $\mathrm{pH}$ 5.8 (Duffy et al., 2002). In previous work with Gap26/27, mimetic peptides of the CL like Gap19 were used as inactive control peptides for GJ studies. In line with this, Gap19 had no acute effect on $\mathrm{Cx} 43$ GJs while it slightly promoted GJ coupling when applied for 24 hours or more (Wang et al., 2013c). Similar findings have been reported for L2 peptide, which decreases the frequency of transitions from the main to the residual state of GJ channels (Seki et al., 2004). Surprisingly, it was found that Gap19, like L2 peptide, inhibited Cx43 HCs (Wang et al., 2013c). They further demonstrated by surface plasmon resonance experiments that Gap19 interacted with the CT and that CT9 peptide inhibited Gap19 HC inhibition in a dose-dependent manner, indicating that Gap19 prevents CL interaction with the CT9 region. As explained previously, CT-CL interaction is necessary for $\mathrm{Cx} 43 \mathrm{HCs}$ to become available for opening (see section II.E.5). As a result, Gap19 prevention of CT-CL interaction will bring $\mathrm{Cx} 43 \mathrm{HCs}$ into a state where they are unavailable for opening. Interaction of Gap19 with the CT target is characterized by a $K_{\mathrm{d}}$ of $\sim 2.5 \mu \mathrm{M}$. Although the CT9 domain is a target of Gap19, other CT sites may also be involved, as was observed with L2 peptide in the context of GJ coupling (see section II.E.2). Gap19 has some intrinsic membrane permeability but coupling of Gap19 to the TAT translocation sequence strongly increases its membrane permeability; experiments with TAT-Gap19 indicated an $\mathrm{IC}_{50}$ of $\sim 7 \mu \mathrm{M}$, i.e., close to the $K_{\mathrm{d}}$ for its interaction with the CT. Based on loss of channel function with the Cx43 I130T mutant (Shibayama et al., 2005) (Fig. 1), a mutation associated with ODDD (see section VII), an I130A mutant Gap19 version was tested and found to be inactive and thus useful as a control peptide (Wang et al., 2013c). Gap19 was tested on $\mathrm{Cx} 40 \mathrm{HCs}$ and Panx1 channels, on which it had no effects (Wang et al., 2013c). Of note, Gap19 contains several charged residues (4 Lys and $1 \mathrm{Glu}$ ) on a total of nine amino acids and its interaction with the CT 
may thus involve substantial electrostatic interactions. The interaction spectrum of the much longer L2 peptide (26 amino acids) will certainly be more elaborate and may ascertain specificity. L2 does not enter the cells spontaneously and needs to be coupled to a translocation motif such as TAT; mutant H126K/I130N L2 is the corresponding inactive control peptide (Ponsaerts et al., 2010). Interestingly, Gap19 has been used to investigate the role of $\mathrm{Cx} 43 \mathrm{HCs}$ in isoproterenol-induced cardiac arrhythmias, demonstrating a drastic improvement of isoproterenol-challenged animal mortality (González et al., 2015). Work with this peptide in mice has furthermore demonstrated it modestly reduces infarct size after myocardial ischemia/reperfusion (Wang et al., 2013c). TAT-linked Gap19 was shown to impair spatial short-term memory when injected into mice brain ventricles (Walrave et al., 2016). TAT-linked L2 peptide potently inhibited norepinephrine-induced vasoconstriction of rat small mesenteric arteries (Bol et al., 2016) and was demonstrated to inhibit fear memory consolidation when injected into rat basolateral amygdala (Stehberg et al., 2012).

Just like $\left[\mathrm{Ca}^{2+}\right]_{i}$, proinflammatory cytokines and metabolic inhibition distinctly influence HCs and GJs, this also appears to be the case for Gap19 and L2 peptides. Taking into account that both peptides interfere with CT-CL interaction and are identical to the sequence of domains crucial for this interaction, this suggests that the process of docking and GJ formation induces conformational changes that switch the outcome of CT-CL interaction from necessary for HC opening to inhibition of GJ.

Interestingly, the L2 peptide has been further explored in terms of its pharmacophore for interacting with the $\mathrm{Cx} 43 \mathrm{CT}$, with the RXP-E motif appearing as an interesting starting point from which other peptides and petidomimetics have been developed (see section II. E.2) (Shibayama et al., 2006b; Lewandowski et al., 2008; Verma et al., 2009). In particular, the linear peptide RRNYRRNY, the cyclic peptide CyRP-71, and the peptidomimetic molecule ZP2519 were demonstrated to target the $\mathrm{Cx} 43 \mathrm{CT}$ and to prevent $\mathrm{Cx} 43$ based GJ closure under low $\mathrm{pH}$ conditions (Verma et al., 2009,2010). These substances are of potential translational value for preventing postischemic GJ closure. Moreover, these molecules are potential HC blockers and may thus have two-sided actions directed at preventing GJ closure as well as inhibiting HC opening. Interestingly, RRNYRRNY was recently demonstrated to act as a $\mathrm{Cx} 43 \mathrm{HC}$ blocker, not only of plasma membrane HCs but also of HCs in subsarcolemmal mitochondria (Gadicherla et al., 2017). As a result, RRNYRRNY displays three levels of action on $\mathrm{Cx} 43$ based channels: prevention of GJ closure, inhibition of $\mathrm{HCs}$ in the plasma membrane, and inhibition of mitochondrial HCs (mitochondrial connexin channels are further discussed in section V.A).

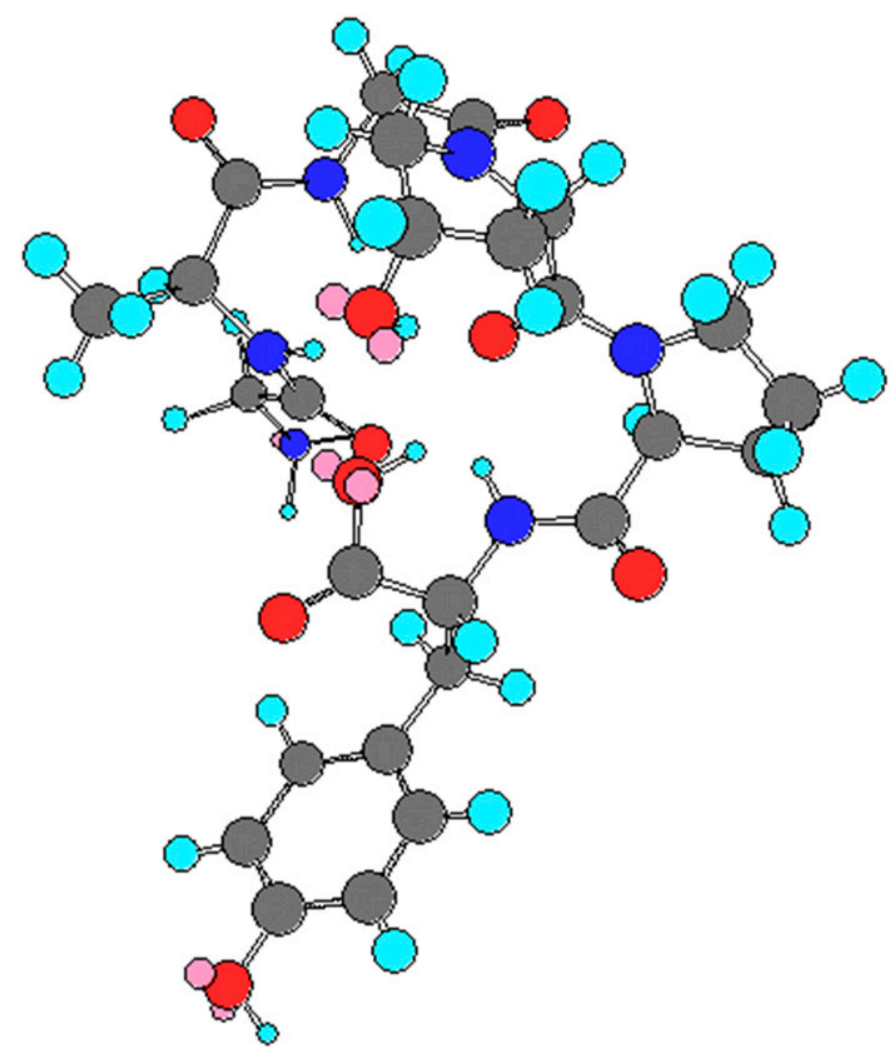

Fig. 3. Chemical structure of AAP10.

2. JM2 Peptide. Recently, the Gourdie group reported that JM2 peptide, composed of 15 amino acids in the juxtamembrane region of the $\mathrm{Cx} 43 \mathrm{CT}$ (see Fig. 1), inhibited ATP release triggered by low extracellular $\mathrm{Ca}^{2+}$ in human microvascular endothelial cells (Calder et al., 2015). The peptide overlaps with the crucial juxtamembrane (JM) domain (amino acids 234-243, indicated in Fig. 1) that functions as a microtubule binding site, which is part of a larger domain stretching from amino acid 228 to 263 (Giepmans et al., 2001). JM2 peptide was tested on hemichannel ATP release, but its effects on gap junctions were not investigated.

3. CT9 Peptide. As discussed earlier, CT9 peptide removes the high $\left[\mathrm{Ca}^{2+}\right]_{\mathrm{i}}$ brake on $\mathrm{Cx} 43 \mathrm{HC}$ opening (see section II.E.5). As a result, CT9 acts as a promoter of $\left[\mathrm{Ca}^{2+}\right]_{\mathrm{i}}$-linked HC opening (Fig. 2B), as illustrated recently in vascular smooth muscle cells (Bol et al., 2016). CT10, which contains an additional Ser (Ser-373), has the same effect (Ponsaerts et al., 2010), but CT9 is the smallest active sequence (the Ser was added as a linker to the TAT translocation motif). Moreover, CT9 $\Delta$ I peptide that lacks the last CT-located Ile (Ile-382 in the Cx43 protein) and therefore does not compete with $\mathrm{Cx} 43-\mathrm{ZO}-1$ interaction (see section II.B.3) still displays its HC activity-enhancing effect (De Bock et al., 2012). Similar to Gap19, L2, and RRNYRRNY, CT9 has its target inside the cell (L2/Gap19 sequences on the $\mathrm{CL}$ ) and needs to be fused to a translocation sequence to improve its membrane permeation (TAT-CT9). 
$\alpha \mathrm{CT} 1$ is another form of CT9 peptide, which is linked to the antennapedia membrane translocation sequence instead of the TAT sequence. Interestingly, $\alpha \mathrm{CT} 1$ promotes $\mathrm{HC}$ incorporation into GJs and increases plaque size by facilitating $\mathrm{HC}$ disconnection from ZO-1-linked cytoskeletal elements as a result of competition between the peptide and the endogenous $\mathrm{Cx} 43$ CT9 motif (Hunter et al., 2005; Palatinus et al., 2012; Rhett et al., 2013) (see section II.B.3). $\alpha \mathrm{CT} 1$ reduces inducible arrhythmias after cryoinjury applied to the left ventricle in the heart and prevents $\mathrm{Cx} 43$ lateralization/remodeling; it also increases $\mathrm{Cx} 43$ phosphorylation at Ser-368 in a PKC $\varepsilon$-dependent manner (O'Quinn et al., 2011). These effects are exerted not within seconds per minute characteristic for CT9 effects on $\mathrm{HC}$ gating but on a time scale of hours that is characteristic for the cardiac $\mathrm{Cx} 43$ life cycle. As a result CT9, fused to either TAT or the antennapedia sequence, has two effects: within minutes it promotes $\left[\mathrm{Ca}^{2+}\right]_{\mathrm{i}^{-}}$ linked HC opening, whereas within hours it promotes $\mathrm{HC}$ incorporation into GJs, thereby reducing the $\mathrm{HC}$ pool in the plasma membrane. If CT9 is used with the purpose of promoting $\mathrm{HC}$ incorporation into GJs (longterm effect to improve GJ coupling), it is advisable to consider combining its administration together with an $\mathrm{HC}$ blocker to prevent the potentially inappropriate acute HC-opening effect of CT9-based peptides.

4. Note on Hemichannel-Targeting Strategies. Currently Gap19, L2, and RRNYRRNY peptide (Fig. 1) are the only known molecules that inhibit $\mathrm{Cx} 43 \mathrm{HCs}$ without inhibiting GJs. Peptide5 is an HC blocker at low (5 $\mu \mathrm{M})$ concentrations that blocks GJs at higher concentrations; Gap26/27 inhibit HCs within minutes, with delayed effects on GJs (hours). Two other (nonpeptide) compounds namely boldine (Hernandez-Salinas et al., 2013) and D4 (Cea et al., 2016b) have been reported to act as connexin HC blockers. Boldine is an alkaloid extracted from the boldo tree but the chemical identity of D4 was not revealed. Recently, Yi et al. (2017) demonstrated that boldine inhibited HCs but not GJs in astrocytes at concentrations of 0.1 and $0.5 \mathrm{mM}$ in cultures and acute brain slices. These authors furthermore showed that long-term in vivo boldine administration (12 weeks, administered in the drinking water) inhibited HCs but not GJs; in APP/PS1 mice such treatment reduced $\left[\mathrm{Ca}^{2+}\right]_{\mathrm{i}}$ elevation in astrocytes, tempered gliotransmitter release and mitochondrial superoxide ion load, and reduced dystrophic neurite counts (Yi et al., 2017). Amyloid load was not affected and behavior/cognition were not investigated.

Certain glycyrrhetinic acid-based molecules have been claimed to act in an HC-specific manner but there is little convincing evidence to support this claimed selectivity (Takeuchi et al., 2011). Lanthanum ions $\left(\mathrm{La}^{3+}\right)$ block HCs and indeed do not inhibit GJs (Anselmi et al., 2008); however, this ion has manifold other effects, including the inhibition of $\mathrm{Ca}^{2+}$ channels (Mlinar and Enyeart, 1993; Young et al., 2002).

Besides peptides, two ODDD $\mathrm{Cx} 43$ mutants have been characterized to display increased $\mathrm{HC}$ function combined with decreased gap junctional function. These mutants are G138R and G60S (see Fig. 1) (Dobrowolski et al., 2007, 2008; Kozoriz et al., 2013). As such, these mutants are interesting tools to distinguish $\mathrm{HC}$ function from GJ function. The G138R mutant has been used to investigate the consequences of increased $\mathrm{HC}$ function in brain slice experiments (Torres et al., 2012), whereas the G60S mutant was demonstrated to increase infarct size in animal stroke models (Kozoriz et al., 2013). Another interesting mutant not linked to ODDD is R76W, which has impaired GJ function but preserved $\mathrm{HC}$ function $(\mathrm{Xu}$ et al., 2015) and G8V that has preserved GJ function but increased HC opening (Wang et al., 2015a) (Fig. 1).

3. Peptides not Mimicking Connexin Sequences: The Case of AAP10. The most striking and best characterized peptide with pronounced effects on GJ channels, which is not based on a connexin protein sequence, is AAP10 peptide and analogs. AAP10 stands for antiarrhythmic peptide 10 and is derived from a naturally occurring antiarrhythmic peptide isolated from bovine atria, which enhanced the synchronization of cultivated clusters of spontaneously beating embryonic chicken cardiomyocytes (Aonuma et al., 1980). Starting from this original antiarrhythmic peptide, a number of synthetic derivatives was developed, among which AAP10 (GAG-4Hyp-PY) (illustrated in Fig. 3) was most active and chosen as lead substance (Grover and Dhein, 2001). The hypothesis that "enhanced synchronization of cardiomyocyte clusters" may represent an increase in intercellular coupling could be verified: simulated ischemia (hypoxia combined with glucose deficit) leads to a reduction in conduction velocity in guinea pig papillary muscles that could be prevented by $1 \mu \mathrm{M}$ AAP10 added to the bath solution (Müller et al., 1997a). Furthermore, it was found that in dual whole cell patch clamp experiments, concentrations of 10 or $50 \mathrm{nM}$ AAP10 lead to enhanced electrical coupling between pairs of cardiomyocytes without affecting the sodium, calcium, potassium, or other transmembrane ionic currents (Müller et al., 1997a,b; Weng et al., 2002; Hagen et al., 2009). Similarly, enhanced coupling was also observed with rotigaptide, a peptide that is further discussed below (Jørgensen et al., 2005; Clarke et al., 2006). Further experiments showed that the positive effect of AAP10 on electrical coupling was increased if cells were partially uncoupled: cells exposed to low $\mathrm{pH}$ (6.5 via $\mathrm{CO}_{2}$ ) exhibit reduced GJ conductance that was prevented by pretreatment with $50 \mathrm{nM}$ AAP10 and, moreover, could also be reversed by treatment with AAP10 (Hagen et al., 2009). Besides electrical coupling, AAP10 also increased metabolic GJ coupling as evident from increased dye transfer (Hagen et al., 2009). The effects of AAP10 on GJs could be demonstrated in rat 
(Hagen et al., 2009), guinea pig (Müller et al., 1997a,b; Weng et al., 2002), rabbit (Dhein et al., 1994; Jozwiak and Dhein, 2008), and human (Hagen et al., 2009) cardiomyocytes. Experiments using HeLa cells stably transfected with either $\mathrm{Cx} 43, \mathrm{Cx} 40$, or $\mathrm{Cx} 45$, i.e., the typical cardiac connexins, showed that AAP10 acts on GJs via effects on $\mathrm{Cx} 43$ or $\mathrm{Cx} 45$ but not via $\mathrm{Cx} 40$ (Easton et al., 2009; Hagen et al., 2009).

Because peptides often are biologically unstable, further research was focused on the structural chemistry of these peptides and on development of a radioligand binding assay to allow the development of nonpeptide drugs. Structure-activity relations together with molecular modeling, and two-dimensional NMR spectroscopy revealed that AAP10 has a semicyclic structure like a horseshoe, which is due to the two Pro residues (Dhein et al., 2010; Grover and Dhein, 1998, 2001). Moreover, it could be shown that $\left[{ }^{14} \mathrm{C}\right] \mathrm{AAP} 10$ binds to a membrane protein of rabbit cardiomyocyte membranes (Dhein et al., 2001) with a $K_{\mathrm{d}}$ in the order of $0.3-0.9 \mathrm{nM}$ and maximum binding $B_{\max }$ in the order of $\sim 42.5 \mathrm{pmol} / \mathrm{mg}$ (Jozwiak et al., 2012). The finding of the semicyclic structure and the identification of the essential chemical moieties (Grover and Dhein, 1998, 2001) led to the idea of replacing some of the amino acids by D-amino acid versions. To maintain certain groups at their positions, the order of these amino acids had to be reversed, which, using a retro-all-D amino acid design of the AAP10 template, led to ZP123 (YP-4Hyp-GAG) also named rotigaptide (Kjølbye et al., 2003; Xing et al., 2003). Due to the D-amino acids, its in vivo half-life is longer than that of AAP10. As AAP10, the D-amino acid analog rotigaptide enhanced GJ communication but did not bind to other transmembrane ion channels (Haugan et al., 2005). Further reduction to the pharmacophore allowed synthesis of the dipeptide danegaptide also called ZP1609 (Skyschally et al., 2013) and of the nonpeptide drug Gap134 [(2S,4R)-1-(2-aminoacetyl)-4benzamido-pyrrolidine-2-carboxylic acid hydrochloride] (Butera et al., 2009).

Until this point, we have only considered the effects of these drugs on GJs, but the question arises whether antiarrhythmic peptides really exert antiarrhythmic effects and against which type of arrhythmia they may be effective. Early considerations using computer simulations about the relationship of intercellular coupling and arrhythmia started with the idea that reduced coupling might unmask local inhomogeneities in action potential duration (APD), resulting in a dispersion of APDs (Lesh et al., 1989; Müller and Dhein, 1993). Together with slowed conduction these local differences in refractoriness could lead to reentrant arrhythmia (for more detail see section VI). Based on this hypothesis, 256 electrode mapping was performed using isolated rabbit hearts, which demonstrated, in good accordance to the computer simulations, that under control conditions AAP10 significantly reduced dispersion in APD
(Dhein et al., 1994) and that regional ischemia results in alterations of the spread of activation that were reduced by AAP10 (Dhein et al., 1994). In addition, ischemiainduced slowing of conduction in the ischemic border zone was antagonized by AAP10 (Jozwiak and Dhein, 2008). Accordingly, it was also shown that AAP10 could significantly decrease the incidence of ventricular tachycardia and ventricular fibrillation in acute ischemia (coronary ligation) (Ni et al., 2015; Sun et al., 2015a). Similarly, rotigaptide (ZP123) also reduced the incidence of ventricular fibrillation and ventricular tachycardia in acute coronary ischemia in various animal models (Hennan et al., 2006; Kjølbye et al., 2008; $\mathrm{Su}$ et al., 2015), prevented ischemia-induced conduction velocity slowing (Shiroshita-Takeshita et al., 2007) and suppressed arrhythmia in volumepressure overload heart failure (Liu et al., 2014). Spiral wave reentry circuits became destabilized by enhancement of GJ coupling (Takemoto et al., 2012). As AAP10, rotigaptide also reduced APD dispersion (Dhein et al., 2003). Interestingly, AAP10 additionally reduced the inducibility of ventricular fibrillation in healed myocardial infarction (Ren et al., 2006). Of note, rotigaptide also reduced infarct size (Haugan et al., 2006; Hennan et al., 2006), although others did not observe this effect (Xing et al., 2003). Infarct size reduction has also been found with danegaptide (ZP1609) (Skyschally et al., 2013). Recent work demonstrated ZP1609 prevents cardiomyocyte hypercontracture after ischemiareperfusion by acting on mitochondria (Boengler et al., 2017); this particular effect was, however, not linked to $\mathrm{Cx} 43$. AAP10 has furthermore been demonstrated to prevent drug-induced torsade de pointes arrhythmia and early afterdepolarizations (Quan et al., 2007, 2009; Ruan et al., 2014). In cardiac preparations uncoupled with lysophosphatidic acid, AAP10 antagonized ventricular tachycardia induced by programmed S1S2 stimulation (Zhou et al., 2011). In vivo, the inducibility of ventricular fibrillation by the plant toxin aconitine was significantly attenuated by AAP10 (Dhein et al., 2001).

In contrast, AAP10 did not affect burst stimulationinduced atrial fibrillation (Haugan et al., 2004). Similarly, rotigaptide did not prevent atrial tachyarrhythmia development in a chronic volume overload rabbit model (Haugan et al., 2006), whereas in a canine mitral regurgitation model, rotigaptide was effective in reducing atrial fibrillation inducibility (Guerra et al., 2006) as well as against atrial conduction slowing induced by metabolic stress (Haugan et al., 2006) or atrial stretch (Ueda et al., 2014). This was also found for Gap134 (Rossman et al., 2009). AAP10 could not reverse diabetes mellitus-induced conduction slowing in Zucker diabetic fatty rats (Olsen et al., 2013); this may be linked to the fact that this type of conduction slowing is probably due to enhanced fibrosis rather than resulting from functional electrophysiological changes 
and may therefore be more difficult to reverse. Taken together, most researchers found evidence for antiarrhythmic effects of AAP10 and related peptides against ischemia-induced ventricular tachyarrhythmias, while efficacy in atrial fibrillation seems to depend on the model used and the type of atrial fibrillation. The efficacy of antiarrhythmic peptides seems to be highest in partially uncoupled tissue if uncoupling is induced by hypoxia, ischemia, or acidosis.

This leads to the question of the biochemical mechanisms of action of antiarrhythmic peptides. First of all, it has been shown that AAP10 and rotigaptide binds to a membrane protein with a $K_{\mathrm{d}}$ in the range of $0.1-0.9 \mathrm{nM}$ $\left[\left[{ }^{14} \mathrm{C}\right] \mathrm{AAP} 10\right.$ (Dhein et al., 2001; Jozwiak et al., 2012); $\left[{ }^{125} \mathrm{I}\right]$ di-I-AAP10 (Jørgensen et al., 2005)]. A 200-kDa membrane protein could be isolated from cardiac tissue by affinity chromatography and cross-linking techniques (Weng et al., 2002), but its identity was not determined. Regarding the subsequent signal transduction cascade, the antagonization of the AAP10 effects by GDP- $\beta$ S is in favor of the idea that G-proteins are involved in the signaling process (Weng et al., 2002). Furthermore, the peptides activate protein kinase C $\alpha$ [AAP10 (Dhein et al., 2001; Weng et al., 2002); rotigaptide (Dhein et al., 2003)]. The effect of AAP10 on GJIC and on Cx43 phosphorylation could be completely inhibited by blocking PKC (Weng et al., 2002; Easton et al., 2009), indicating that PKC is critically involved in the signal transduction process. Consistently, several groups found enhanced phosphorylation of $\mathrm{Cx} 43$ after incubation of the cells/tissue with antiarrhythmic peptides [AAP10 (Dhein et al., 2001; Weng et al., 2002; Quan et al., 2007, 2009; Wang et al., 2007b; Easton et al., 2009; Sun et al., 2015a); rotigaptide (Kjølbye et al., 2008; Su et al., 2015)]. In contrast, others did not observe enhanced Cx43 phosphorylation with rotigaptide (Clarke et al., 2006). However, within the first 30 minutes of ischemia, Ser-306, Ser-297, and Ser-368 of Cx43 are dephosphorylated, whereas Ser-330 is phosphorylated. The dephosphorylation of Ser-297 and Ser-368 can be prevented by rotigaptide (Axelsen et al., 2006). Cx43 synthesis was not affected by rotigaptide (Liu et al., 2014). In contrast, AAP10 led to an increase in Cx43 mRNA expression (Easton et al., 2009).

More detailed analysis revealed that the effects of AAPs on Cx43 phosphorylation were enhanced in ischemic tissue and attained a maximum in the ischemic center while less pronounced in nonischemic tissue (Jozwiak and Dhein, 2008), which may explain the controversial findings cited above. Accordingly, others found that the binding site density for AAP10 was enhanced during metabolic stress (Jørgensen et al., 2005). Thus, one might conclude from these finding that AAPs prevent from dephosphorylation rather than induce active phosphorylation.
Phosphorylation of $\mathrm{Cx} 43 \mathrm{CT}$ can alter the single channel conductance (Takens-Kwak and Jongsma, 1992; Moreno et al., 1994; Kwak et al., 1995a; Kwak and Jongsma, 1996), but also is critically involved in controlling the transfer and insertion of $\mathrm{Cx} 43$ into the membrane (involving the tubulin apparatus) and in the removal of $\mathrm{Cx} 43$ from the membrane (Lampe and Lau, 2000; Solan and Lampe, 2007; Saidi Brikci-Nigassa et al., 2012). Accordingly, two groups found that $\mathrm{Cx} 43$ density in the membranes is reduced in ischemia and can be preserved by AAPs (Jozwiak and Dhein, 2008; Sun et al., 2015a). Moreover, it was shown by these authors that the reduction of $\mathrm{Cx} 43$ and its inhibition by AAP10 was highest at the cell poles. Interestingly, AAP10 led to a higher $\mathrm{Cx} 43$ density at points of cellcell contact (Easton et al., 2009), which would be in line with the assumption that the incorporation into the membrane might be enhanced. The mechanism of action of the AAPs is summarized in Fig. 4.

Regarding clinical safety, rotigaptide was evaluated in a phase I study in 200 healthy subjects and found to be safe (Kjølbye et al., 2007). Taken together, AAPs have been demonstrated to possess a pharmacological potential for the treatment of ischemia- or hypoxia-linked arrhythmias related to cellular uncoupling via $\mathrm{Cx} 43$ or $\mathrm{Cx} 45$. On the background of the observations that GJs also control growth and differentiation of cells, this approach may also be worth investigating in the area of cancer (Salameh and Dhein, 2005).

\section{Connexins in Vascular Disease}

Atherosclerosis, a progressive inflammatory disease of large and medium-sized arteries, is the number one killer worldwide. The main complications of atherosclerosis, namely ischemic heart disease and stroke, are the world's first and third leading causes of death, representing $28.5 \%$ of all-cause mortality (GBD 2013 Mortality and Causes of Death, 2015). The disease involves the formation of plaques in the intima of arteries that are characterized by a dysfunctional endothelium, leukocyte and lipid accumulation, cell death, and fibrosis (Fig. 5). Atherosclerotic plaques develop predominantly at arterial locations where regular (high) laminar blood flow is disturbed, i.e., arterial bifurcations and branch points (Kwak et al., 2014). The most severe clinical events follow the rupture of a plaque (Fig. 6), which exposes prothrombotic material inside the plaque to the blood and causes sudden thrombotic occlusion of the artery at the site of disruption (Hansson et al., 2015). Various connexins have been shown to be involved in the initiation and progression of atherosclerosis. Furthermore, these proteins may also influence thrombus formation and stabilization.

Treatment of acute coronary atherothrombosis consists of procedures that allow the rapid return of blood flow to the ischemic zone of the myocardium to rescue 


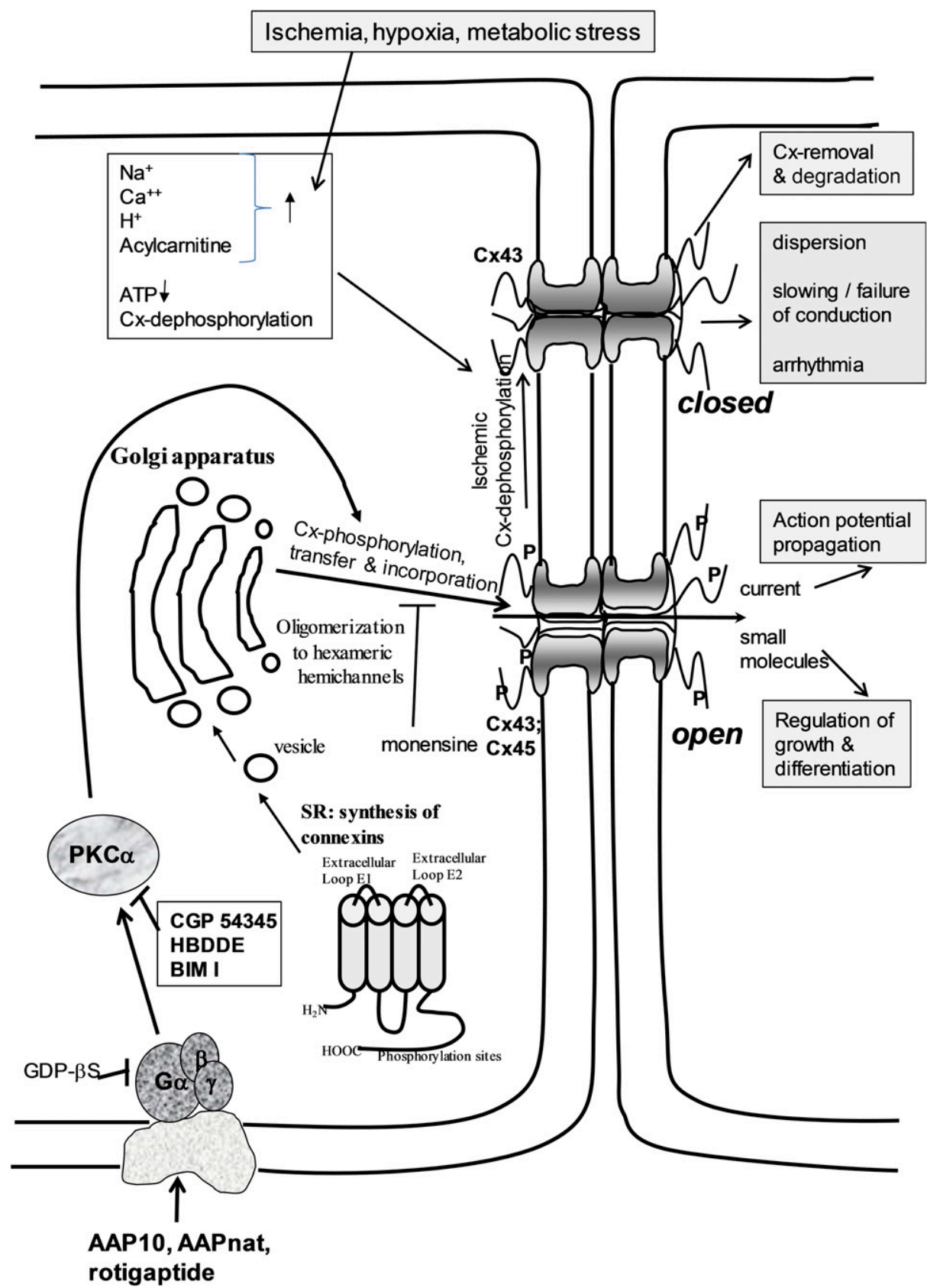

Fig. 4. Synthesis, transport, and membrane incorporation of cardiac connexins and mechanisms of ischemic damage leading to GJ closure, connexin dephosphorylation, and connexin-removal from the membrane. Molecular mechanisms and effects of antiarrhythmic peptides on GJ channels formed by $\mathrm{Cx} 43$ or $\mathrm{Cx} 45$ are also illustrated. GDP- $\beta \mathrm{S}$, guanosine-5'-[ $\beta$-thio]diphosphate trilithium salt (nonhydrolyzable GDP analog); PKC $\alpha$, protein kinase C $\alpha$; CGP 54345, PKC inhibitor (inhibits only PKC $\alpha$ ); HBDDE 2,2',3,3',4,4-hexahydroxy-1'-biphenyl-1-6,6'-dimethanoldimethylether, inhibitor of PKC $\alpha$ and PKC $\gamma$; BIM I, bisindolyl-maleimide I, inhibitor of PKC $\alpha ; \beta \mathrm{I}, \beta \mathrm{II}, \gamma, \delta$, and $\varepsilon$; SR, sarcoplasmic reticulum; AAPnat, natural antiarrhythmic peptide (H-Gly-Pro-Hyp-Gly-Ala-Gly). Modified from Dhein et al. (2010).

heart muscle. However, reperfusion may paradoxically lead to further complications involving acceleration of cardiac cell death, diminished contractile function, and arrhythmias. The involvement of cardiomyocyte connexins in reperfusion injury will be described in section IV.C; here we will summarize recent evidence indicating an involvement of connexins in the inappropriate inflammatory response in the microcirculation that may be at the basis of ischemia-reperfusion injury (Bulluck et al., 2016).
Restenosis is the pathophysiological process involving inflammatory activation and intimal smooth muscle cell accumulation that occurs in about $10 \%$ of patients submitted to revascularization procedures of coronary, carotid, and peripheral arteries. It is in fact an excessive healing reaction of the vascular wall subjected to balloon angioplasty and endovascular stent implantation (Chaabane et al., 2013). Drug-eluting stents and the more recent drug-eluting balloons have significantly reduced but not eliminated the incidence of restenosis. 
A

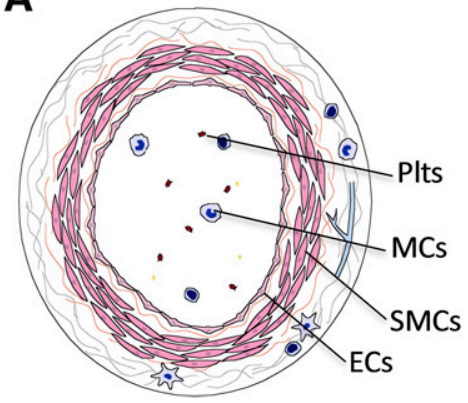

Healthy artery

ECs: Cx37, Cx40

SMCs: $\mathrm{Cx} 43$

MCs: Cx37

Plts: Cx37 (Cx40, Cx43)
B

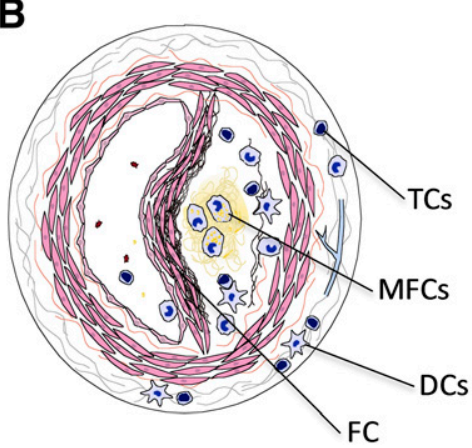

Stable atherosclerotic lesion

ECs (covering lesion): $\mathrm{Cx} 43$

SMCs (intimal): Cx43

SMCs (media): Cx43 (Cx37)

MFCs: Cx37, Cx43

TCs: $\mathrm{Cx} 43$

DCs: $\mathrm{Cx} 43$

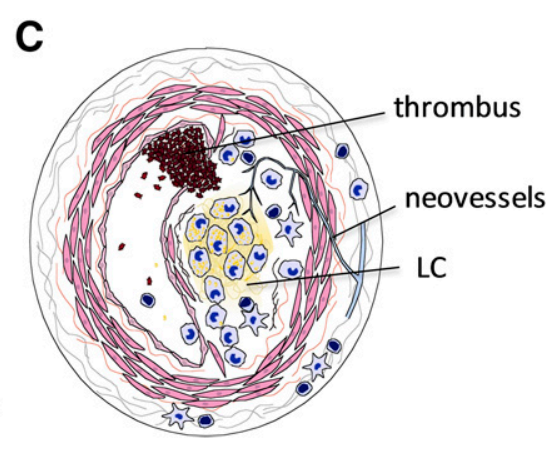

Vulnerable atherosclerotic lesion

ECs (covering lesion): Cx43
SMCs (intimal): Cx43
SMCs (media): Cx43 (Cx37)
MFCs: Cx37, Cx43
TCs: Cx43
DCs: $C \times 43$

ECs (covering lesion): $\mathrm{Cx} 43$

SMCs (intimal): $\mathrm{Cx} 43$

SMCs (media): Cx43 (Cx37)

TCs: $\mathrm{C} \times 43$

DCs: $\mathrm{Cx} 43$

Fig. 5. Connexin expression in healthy arteries (A), stable atherosclerotic plaques with a thick fibrous cap (B), and after rupture of vulnerable lesions. Connexin expression is represented according to cell type. DCs, dendritic cells; ECs, endothelial cells; FC, fibrous cap; LC, lipid core MCs, monocytes; MFCs, macrophage foam cells; Plts, platelets; SMCs, smooth muscle cells; TC, T cells.

The role of connexins in this clinically still relevant problem will be discussed.

\section{A. Connexins in Atherosclerosis}

There is growing evidence that connexins participate in the development of atherosclerotic disease. Early support for this hypothesis came from studies analyzing atherosclerotic lesions at different disease stages in specimens of human, rabbit, or mouse origin. First, $\mathrm{Cx} 43$ is generally absent in the endothelium of large arteries, but it has been found in endothelial cells at the shoulder region of advanced atherosclerotic plaques (Fig. 5) (Kwak et al., 2002). Although endothelial Cx43 expression may be induced by cytokines like TGF- $\beta$ that are present in excess in an atherosclerotic environment (Larson et al., 2001), Cx43 expression may already have been upregulated at this specific arterial location before the atherosclerotic lesion formed. Support for the latter premise comes from the fact that abundant $\mathrm{Cx} 43$ expression has been observed in rat aortic endothelial cells localized at the downstream edge of the ostia of branching vessels and at flow dividers, regions known to experience disturbed blood flow (Gabriels and Paul, 1998). Subsequently, a causal relation between the induction of endothelial $\mathrm{Cx} 43$ and disturbed (or oscillatory) flow has been established in various in vitro studies (DePaola et al., 1999; Kwak et al., 2005; Feaver et al., 2008). Secondly, a high level of $\mathrm{Cx} 43$ expression has been found in intimal macrophages and smooth muscle cells of young atheroma, whereas $\mathrm{Cx} 43$ expression is downregulated in intimal smooth muscle cells of more advanced lesions (Fig. 5) (Polacek et al., 1993, 1997; Blackburn et al., 1995; Kwak et al., 2002).
Interestingly, the oxidized phospholipid derivative POVPC reduces $\mathrm{Cx} 43$ levels of vascular smooth muscle cells, enhances its phosphorylation at Ser-279/282, and increases smooth muscle cell proliferation both in vitro and in an atherosclerotic mouse model in vivo (Johnstone et al., 2009). Finally, Cx37 and Cx40 levels are also modified during the course of atherosclerotic disease in human and mice (Kwak et al., 2002). Thus, Cx40 and Cx37 disappear from endothelial cells covering advanced atherosclerotic lesions, and Cx37 expression is enhanced in macrophage foam cells (Fig. 5). Moreover, long-term hyperlipidemia (a well-known atherogenic condition) reduces endothelial $\mathrm{Cx} 37$ and Cx40 expression in mouse aorta, an effect that could be reversed only for $\mathrm{Cx} 37$ by 1 week of treatment with simvastatin, a cholesterol-lowering drug (Yeh et al., 2003). In addition, endothelial Cx37 is downregulated in response to disturbed flow (Pfenniger et al., 2012b). Altogether, these studies brought the idea that connexin expression or posttranslational modifications in connexins might evolve in atherosclerotic plaques over time, depending on the stage of the lesion and might thus affect atherogenesis.

As germ-line loss of $\mathrm{Cx} 43$ is lethal (Reaume et al., 1995), the role of $\mathrm{Cx} 43$ in atherosclerosis was first studied in $C x 43^{+/-}$mice crossed with atherosclerosissusceptible low-density lipoprotein (LDL) receptordeficient $\left(L d l r^{-/-}\right)$mice fed a high-cholesterol diet. Initial studies on $C x 43^{+/-} L d l r^{-/-}$mice revealed that $\mathrm{Cx} 43$ has an overall atherogenic effect, and that reducing $\mathrm{Cx} 43$ might be beneficial by both reducing plaque burden as well as stabilizing the lesions (Kwak et al., 2003). However, the exact scenario by which 

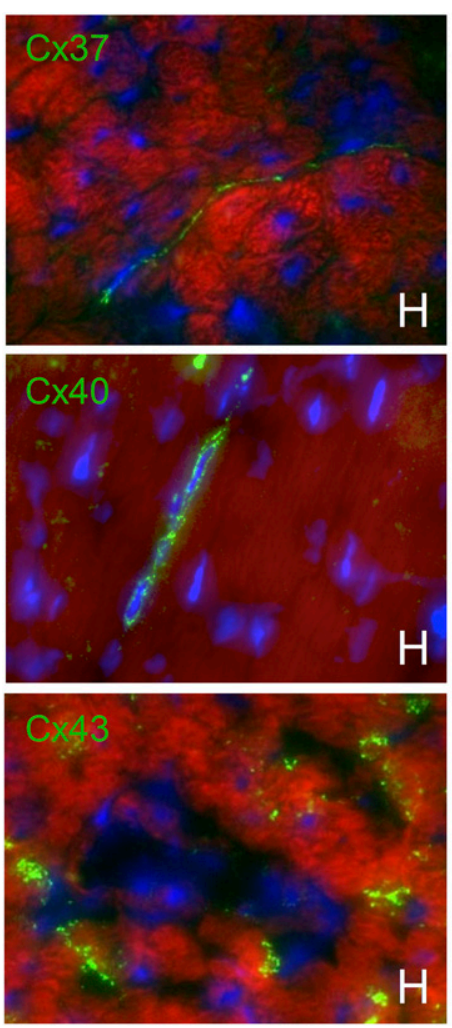
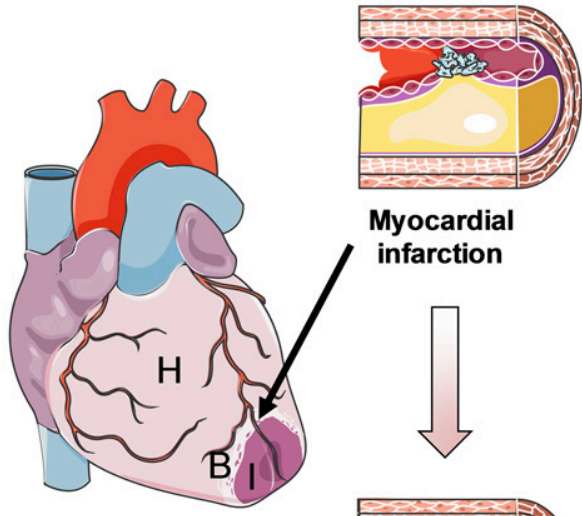

Myocardial

infarction
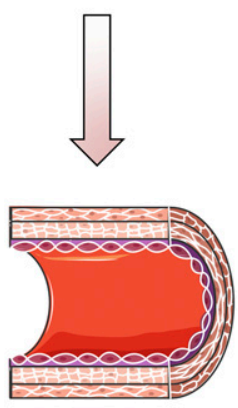

Reperfusion
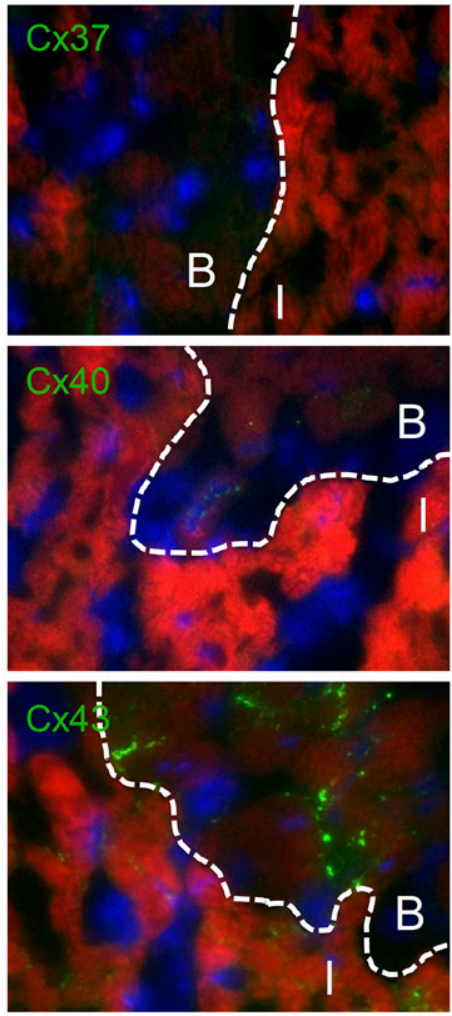

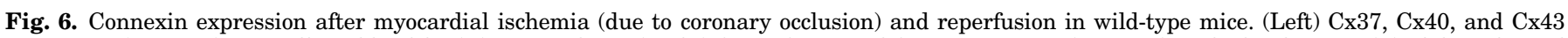

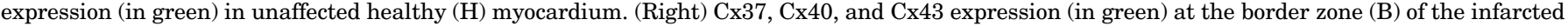
area (I). Tissue is counterstained with Evans Blue (in red), and nuclei are stained with DAPI (in blue).

ubiquitous reduction in $\mathrm{Cx} 43$ leads to this dual benefit remained uncertain due to $\mathrm{Cx} 43$ expression in multiple atheroma-associated cell types (Wong et al., 2003). To examine specifically the role of $\mathrm{Cx} 43$ in immune cells, $\mathrm{Ldlr}^{-1-}$ mice were lethally irradiated and reconstituted with $\mathrm{Cx} 43^{+/+}, \mathrm{Cx} 43^{+/-}$, or $\mathrm{Cx} 43^{-/-}$hematopoietic fetal liver cells (Morel et al., 2014b). Intriguingly, the progression of atherosclerosis was lower in $\mathrm{Cx} 43^{+/-}$chimeras compared with $\mathrm{Cx} 43^{+/+}$and $\mathrm{Cx} 43^{-/-}$chimeras, and their plaques contained fewer neutrophils. It turned out that chemoattraction of neutrophils, which themselves do not express $\mathrm{Cx} 43$, was reduced in response to supernatant secreted by $\mathrm{Cx} 43^{+/-}$macrophages in comparison with the ones of $\mathrm{Cx} 43^{+/+}$and $\mathrm{Cx} 43^{-/-}$macrophages. Thus, titration of $\mathrm{Cx} 43$ levels in macrophages might regulate their chemoattractant secretion, leading to a reduction in atherosclerosis (Morel et al., 2014b).

In contrast to $\mathrm{Cx} 43, \mathrm{Cx} 40$ protects against atherosclerosis in mice by synchronizing endothelial CD73dependent anti-inflammatory signaling, thus inhibiting leukocyte recruitment to the atherosclerotic lesion (Chadjichristos et al., 2010). Furthermore, loss of Cx37 promotes the development of atherosclerosis in apolipoprotein E-deficient $\left(\right.$ Apoe $\left.^{-/-}\right)$mice. Mechanistically, it appeared that ATP release through Cx37 HCs in monocytes control the initiation of atherosclerotic plaque development by regulating their adhesion
(Wong et al., 2006). Recent in vitro studies show a downregulation of Cx37 expression, inhibition of ATP release, and augmented adhesion of the human monocytic cell line THP-1 by oxidized LDL (Liu et al., 2016). These harmful effects of oxidized LDL could be in part prevented by rutaecarpine, an active component of a Chinese herbal medication (Liu et al., 2016). Finally, Cx37 deletion in apolipoprotein $\mathrm{E}^{-/-}\left(\mathrm{Apoe}^{-/-}\right)$mice not only controls the initiation of atherosclerotic plaque formation but also increases the size of advanced lesions and abrogates the development of a stable plaque phenotype in regions exposed to oscillatory shear stress (Pfenniger et al., 2015), suggesting that local hemodynamic factors may modify the risk for Cx37-related adverse disease outcomes. A recent study examining the effects of lentiviral Cx37 interference on established abdominal aortic plaques in pigs by intravascular ultrasound revealed a reduction in plaque volume in the 8 months after lentiviral transduction, illustrating that not only local hemodynamic factors but also the disease stage itself may influence the outcome of reducing $\mathrm{Cx} 37$ on atherosclerosis burden (Guo et al., 2015). Although these animal studies have revealed important and diverse contribution of vascular connexins to atherogenesis, more work is needed to uncover the roles of these proteins in human disease.

During the past 15 years, a single nucleotide polymorphism (SNP) in the human Cx37 gene (GJA4) has 
been associated in a variety of populations with increased risk for various clinical manifestations of atherosclerosis, such as coronary artery disease, myocardial infarction, and ischemic stroke; however, these studies remained controversial as to which allele carried the risk (see for a review Meens et al., 2012). Consequently, data of multiple studies were extracted by two independent reviewers and a total of 3498 myocardial infarction cases and 3986 controls, as well as 1808 coronary artery disease cases and 1197 controls have been enrolled in a recent meta-analysis (Wen et al., 2014). This meta-analysis demonstrated that the GJA4-1019T allele is a risk factor for myocardial infarction and a protective factor for coronary artery disease. The GJA4 $1019 C>T$ SNP results in a nonconservative Pro to Ser substitution in the CT of Cx37, which has a significant impact on channel function under basal and phosphorylating conditions (Derouette et al., 2009; Morel et al., 2010; Pfenniger et al., 2010). In accordance with the above described studies using Cx37-deficient monocytes, monocytic cells expressing Cx37-319P, encoded by GJA4-1019C, were markedly less adhesive than cells expressing Cx37-319S, encoded by GJA4-1019T (Wong et al., 2006), thus suggesting that $\mathrm{Cx} 37-319 \mathrm{P}$ polymorphic $\mathrm{HCs}$ may function as a protective genetic variant for plaque rupture, leading to myocardial infarction by specifically retarding recruitment of monocytes to human atherosclerotic lesions. In contrast to GJA4 1019C $>$, SNPs located in the promoter region $(-1930 C>T)$ or $3^{\prime}$-untranslated region $(1297 I>D)$ of GJA4, which are presumed to affect Cx37 transcription level or mRNA stability, were not associated with altered risk for coronary artery disease (Han et al., 2008). Moreover, two SNPs in the promoter region of the GJA5 gene, $-44 G>A$ and $+71 A>G$, that were found to significantly reduce $\mathrm{Cx} 40$ transcription (Firouzi et al., 2006) could also not be associated with an altered risk for coronary artery disease or acute myocardial infarction (Pfenniger et al., 2012a; Seifi et al., 2013). Although the latter studies remain to be confirmed in large cohorts or by meta-analyses, they stir up the idea that SNPs affecting connexin channel function may be of greater importance for cardiovascular disease than SNPs affecting connexin expression levels.

\section{B. Connexins in Thrombosis}

Cx37 was the first connexin found in platelets in 2011 (Angelillo-Scherrer et al., 2011), and the additional expression of a number of other connexins [Cx43, Cx40, and Cx32 (Vaiyapuri et al., 2012)] and Panx1 (Taylor et al., 2014; Molica et al., 2015) have been reported since. Transmission electron microscopy has convincingly revealed GJ-like structures between platelets (Vaiyapuri et al., 2012)). Moreover, platelets were shown to display functional Cx37 GJ channels during the aggregation response (Angelillo-Scherrer et al.,
2011). Deletion of Cx37 in mice reduced tail bleeding time, shortened the time to occlusive arterial thrombosis, accelerated mortality in a model of thromboembolism, and enhanced platelet aggregation in response to modest concentrations of the agonists ADP, thrombin, and collagen (Angelillo-Scherrer et al., 2011). Furthermore, promotion of platelet aggregation in vitro was also observed with $\mathrm{Cx} 37$ mimetic blocking peptides. Given the biophysical properties of Cx37 GJ channels, it was hypothesized that these channels synchronize responses in platelets brought in contact during activation by transmitting cAMP to neighboring platelets, thereby functionally inhibiting freshly recruited platelets and limiting further thrombus growth (AngelilloScherrer et al., 2011). Interestingly, other studies report a loss of function in $C x 37^{-1-}$ as well as in $C x 40^{-1-}$ platelets, i.e., fibrinogen binding and $\alpha$-granule secretion were decreased, even under conditions when direct platelet-platelet contacts were excluded (Vaiyapuri et al., 2012, 2013). This implies that the contribution of connexins to platelet aggregation may not be limited to the phase during which platelets come into stable contact with each other but might also occur when only HCs are present. The mechanism by which $\mathrm{Cx} 37 / \mathrm{Cx} 40$ HCs open in the presence of relative high extracellular $\mathrm{Ca}^{2+}$ in blood (which normally keep HCs closed) still remains to be determined. Connexin HCs may allow for the release of ATP from activated platelets, which then might act in an autocrine/paracrine manner on P2X1, inducing a further increase in platelet activation state. The recent discovery of Panx 1 in human platelets is in this respect of particular interest (Taylor et al., 2014; Molica et al., 2015).

\section{Endothelial Connexins in Ischemia- Reperfusion Injury}

It is increasingly recognized that deleterious consequences of ischemia-reperfusion are influenced by the dysfunction of the endothelium and the extent of neutrophil infiltration. Although the crucial role of endothelial connexins in diseases of large arteries is now well established, surprising little attention has been given to the role of endothelial connexins, i.e., $\mathrm{Cx} 40$ and $\mathrm{Cx} 37$, in the response of the microcirculation to ischemia-reperfusion injury (Fig. 6). Interestingly, spontaneous recovery of tissue perfusion after severe unilateral hindlimb ischemia was reduced, and the survival of distal limb tissue was compromised in $C x 40^{-1-}$ mice (Fang et al., 2012). The poor recovery from the ischemic insult in $C x 40^{-1-}$ mice appeared to be due to compromised regulation of tissue perfusion, vascular remodeling, and a prolonged inflammatory response (Fang et al., 2013). Thirty minutes of ischemia followed by 24 -hour reperfusion resulted in increased myocardial infarct size in mice with endothelial-specific deletion of $\mathrm{Cx} 40$; however, no deleterious effects were found in mice with Cx37 deletion (Morel et al., 2014a). 
Mechanistically, endothelial Cx40-dependent cardioprotection appeared to involve CD73 activation that, in turn, limited neutrophil infiltration after cardiac reperfusion (Morel et al., 2014a). The Cx40-CD73 axis may thus represent a novel pharmacological target for controlling the damage associated with reperfusion in coronary disease.

The antiarrhythmic peptide rotigaptide or its analog danegaptide (see section III.B.3) reduce myocardial ischemia-reperfusion injury in procedures on large animals such as open-chest dogs (Hennan et al., 2006) and when given at the moment of reperfusion in pigs (Skyschally et al., 2013; Pedersen et al., 2016). Although the cardioprotective effects of these peptides are typically ascribed to the maintenance of $\mathrm{Cx} 43$-mediated GJ intercellular communication between cardiomyocytes, a recent translational study suggested additional effects of these peptides on the human endothelium. By measuring forearm arterial blood flow in humans, endothelium-dependent vasodilation in response to acetylcholine was attenuated after ischemia-reperfusion in the presence of placebo but not in the presence of intra-arterial rotigaptide (Pedersen et al., 2016).

\section{Connexins in Restenosis}

Acute vascular injury induced by balloon angioplasty is associated with increased $\mathrm{Cx} 43$ expression in neointimal smooth muscle cells and macrophages in various animal models (Polacek et al., 1997; Yeh et al., 1997; Plenz et al., 2004; Wang et al., 2005; Chadjichristos et al., 2006; Li et al., 2012b). Although treatment with the cholesterol-lowering lovastatin or the angiotensinconverting enzyme inhibitor ramipril was shown to decrease both $\mathrm{Cx} 43$ expression and neointima formation after balloon injury, a causal link remained to be proven (Wang et al., 2005; Li et al., 2012b). Thus, hypercholesterolemic $C x 43^{+/-} \mathrm{Ldlr}^{-/-}$mice and $C \times 43^{+/+} \mathrm{Ldlr}^{-/-}$controls were subjected to carotid balloon distension injury in vivo, which induced marked endothelial denudation and activation of medial smooth muscle cells (Chadjichristos et al., 2006). This genetic reduction in $\mathrm{Cx} 43$ expression was found to limit neointima formation after the acute vascular injury by decreasing the inflammatory response as well as reducing smooth muscle cell migration and proliferation. Moreover, endothelial repair was enhanced in mice with reduced Cx43 (Chadjichristos et al., 2006). This suggests that $\mathrm{Cx} 43$ might be an attractive target for local delivery strategies aimed at reducing restenosis. Interestingly, effective knock-down of $\mathrm{Cx} 43$ was recently achieved with a lentiviral vector expressing $\mathrm{Cx} 43-$ targeting shRNA and also resulted in an attenuation of neointima formation after balloon injury in rats (Han et al., 2015). Surprisingly, smooth muscle-targeted knockout of $\mathrm{Cx} 43$ enhanced neointima formation in response to carotid wire or occlusion injury (Liao et al., 2007). Thus, the level to which $\mathrm{Cx} 43$ is reduced as well as the progression of the disease may be of crucial importance for the final outcome. Additional research will be needed before moving on to a translational setting of targeting $\mathrm{Cx} 43$ for the reduction of restenosis.

\section{Connexins in Cardiac Disease}

The heart mainly expresses the connexin isoforms $\mathrm{Cx} 31.9, \mathrm{Cx} 37, \mathrm{Cx} 40, \mathrm{Cx} 43$, and $\mathrm{Cx} 45$, whereby $\mathrm{Cx} 43$ is the most abundant isoform and is mainly found in ventricular cardiomyocytes (Severs et al., 2008) but is also expressed in fibroblasts (McArthur et al., 2015) and stem cells ( $\mathrm{Lu}$ et al., 2012b). Other isoforms like $\mathrm{Cx} 40$ are expressed in the atria of the heart; $\mathrm{Cx} 31$ and $\mathrm{Cx} 45$ in the conductance system; and $\mathrm{Cx} 37, \mathrm{Cx} 40$, and $\mathrm{Cx} 43$ in the coronary circulation. The expression of $\mathrm{Cx} 43$ in cardiomyocytes is affected by many factors, including factors released by activation of the neurohumoral system (Salameh et al., 2013) or immune system (Zhang et al., 2016a) as well as by microRNAs (miRNA; further discussed in section V.B.1). As introduced earlier, connexins have a fast protein turnover with a half-life in the order of 1-2 hours in cardiomyocytes, necessitating specific requirements in the organization of the connexin turnover cycle (see section II.B). As a result, the high level of forward connexin trafficking to the sarcolemma needs to be balanced by a wellorganized degradation machinery that encompasses multiple proteolytic (Beardslee et al., 1998) and lysosomal (Laing et al., 1997) pathways. Forward trafficking of connexins to the sarcolemma is a crucial component of the connexin life cycle in cardiomyocytes, and in the human heart it has been demonstrated that four truncated connexin isoforms with a molecular weight of $\sim 20 \mathrm{kDa}$ play a central role because their ablation arrests trafficking of full-length connexin (Smyth and Shaw, 2013). At the intercalated discs, Cx43 interacts with various scaffolding proteins including ZO-1 and others (see section II.B.3). Transmembrane protein 65 (Tmem65) is another scaffolding protein, which functionally regulates $\mathrm{Cx} 43$ and thereby cellular coupling (Sharma et al., 2015). The end-station of forward trafficking is the perinexus, a zone at the periphery of the nexus that contains the GJ plaques at the intercalated disks (IDs) (Rhett et al., 2011; Rhett and Gourdie, 2012). In the perinexus, $\mathrm{Cx} 43$ arrives as $\mathrm{HCs}$, which then come loose of their ZO-1 binding to become incorporated into the GJs. The perinexus as a defined zone of $\mathrm{HC}$ residency is likely to be the site where $\mathrm{HC}$ opening may occur. $\mathrm{HC}$ opening can be triggered by lowering $\left[\mathrm{Ca}^{2+}\right]_{\mathrm{e}}$, increasing $\left[\mathrm{Ca}^{2+}\right]_{\mathrm{i}}$, connexin dephosphorylation, metabolic inhibition, or hyperosmolar conditions, as demonstrated in isolated ventricular cardiomyocytes by dye uptake and electrophysiological methods (Kondo et al., 2000; John et al., 
2003; Wang et al., 2012a). Within cardiomyocytes, Cx43 is located also in subsarcolemmal mitochondria (see below) and the CT of $\mathrm{Cx} 43$ translocates into the nucleus and inhibits cell proliferation (Dang et al., 2003; Zhao et al., 2015). Cardiac connexins also have extensive nonchannel functions that in many cases link to interactions of the $\mathrm{Cx} 43 \mathrm{CT}$ with various scaffolding and signaling proteins, forming a connexin interactome network or "connexome." Cx43 interacts with proteins related to various biologic processes such as cell cycle, metabolism, signaling, and trafficking (see also section II.C). Importantly, the interactome of $\mathrm{Cx} 43$ is differentially modulated in diseased hearts (for review, see Martins-Marques et al., 2015a). Work of the Delmar group has demonstrated that the $\mathrm{Cx} 43 \mathrm{CT}$ is involved in interactions that influence capture of the microtubule plus end at the ID and thereby facilitate sarcolemmal delivery of $\mathrm{Na}^{+}$channels at the cell end (Agullo-Pascual et al., 2014b; reviewed in Leo-Macias et al., 2016). Thus, alterations in connexin trafficking are likely to influence cell-cell coupling as well as electrical excitability, thereby leading to arrhythmia. Along this line, the $\mathrm{Cx} 43$ interactome partner plakophilin-2, which associates with $\mathrm{Cx} 43$ at the ID has been demonstrated to be involved in arrhythmias associated with the Brugada syndrome in genetically predisposed patients (Cerrone et al., 2014; Agullo-Pascual et al., 2014a). Figure 7 summarizes some of the proposed roles of GJs and HCs in cardiac ischemia-reperfusion injury.

\section{A. Cx43 in Mitochondria}

$\mathrm{Cx} 43$ is detected in cardiomyocyte mitochondria from mouse, rat, porcine, and human origin using antibody-dependent and -independent techniques (Boengler et al., 2005; Miro-Casas et al., 2009; Jovic et al., 2012). The analysis of subsarcolemmal and interfibrillar mitochondria for the presence of $\mathrm{Cx} 43$ shows that $\mathrm{Cx} 43$ is almost exclusively localized in subsarcolemmal mitochondria (Boengler et al., 2009; Sun et al., 2015b). Cx43 is encoded in the nuclear genome and imported into the mitochondria in a heat shock protein 90 , translocase of the outer membrane 20-dependent fashion. $\mathrm{Cx} 43$ is located at the inner membrane of subsarcolemmal mitochondria, with its carboxy terminus oriented toward the intermembrane space (Rodríguez-Sinovas et al., 2006), but may also be present at the outer mitochondrial membrane regulating the release of some intermembrane space proteins (Goubaeva et al., 2007).

In the inner membrane of subsarcolemmal mitochondria, $\mathrm{Cx} 43$ interacts with the apoptosis-inducing factor, the $\beta$-subunit of the electron-transfer protein (Denuc et al., 2016) and the ATP-sensitive potassium channel subunit Kir6.1 (Waza et al., 2014). The amount of mitochondrial $\mathrm{Cx} 43$ declines in cardiomyocytes after the activation of the $N$-methyl-D-aspartate receptor 1 by the enhanced mitochondrial translocation of the matrixmetalloproteinase 9 (Tyagi et al., 2010). In isolated mitochondria of $\mathrm{Cx} 43$-deficient mice hearts, the reduced mitochondrial $\mathrm{Cx} 43$ content is associated with a switch of the mitochondrial NOS isoform and the decrease in mitochondrial nitric oxide formation (Kirca et al., 2015).

In isolated subsarcolemmal mitochondria, chemical cross-linking generates $\mathrm{Cx} 43$-dependent proteincomplexes at a molecular weight corresponding to that of GJ-enriched membranes, possibly representing $\mathrm{Cx} 43$

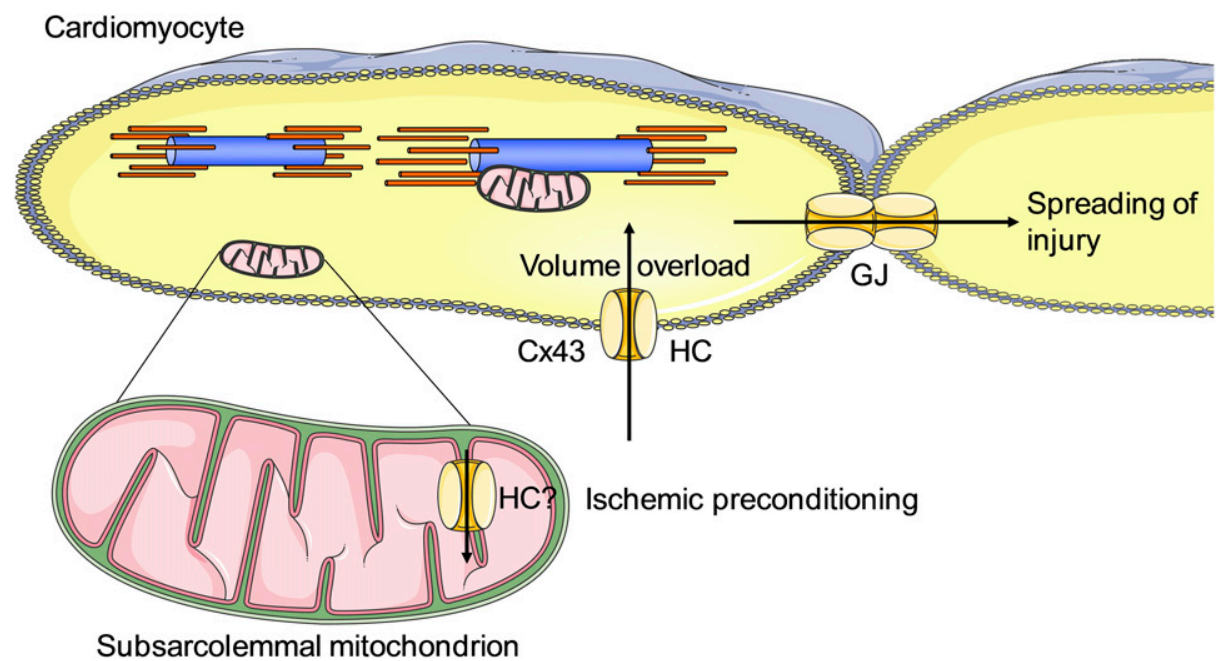

Fig. 7. Roles of $\mathrm{Cx} 43$ in cardiac ischemia-reperfusion. Gap junctions close under ischemic conditions ("healing over" caused by the low $\mathrm{pH}$ and elevated $\left[\mathrm{Ca}^{2+}\right]_{\mathrm{i}}$ ) but substantial coupling may persist after ischemia-reperfusion (Ruiz-Meana et al., 2001). Open GJs may act beneficially by supplying essential metabolites to neighboring cells but may also spread injury signals, causing cell death propagation (reviewed in Decrock et al., 2009b; Michela et al., 2015). GJs have been implicated in the spreading of hypercontracture necrosis in a process mediated by $\mathrm{Na}^{+}$flux through GJs (reviewed in García-Dorado et al., 2004). Uncontrolled hemichannel (HC) opening facilitates ionic fluxes that may lead to cell swelling (Wang et al., 2013c). Cx43 is also present in mitochondria where they are involved in the signaling cascade of ischemic preconditioning, conferring cardioprotective effects (reviewed in Schulz et al., 2007; Miura et al., 2010; Schulz et al., 2015). Mitochondrial Cx43 has been demonstrated to form hexameric structures involved in inner mitochondrial membrane $\mathrm{K}^{+}$fluxes, pointing to functional HCs (Miro-Casas et al., 2009). 
hexamers. Mitochondrial uptake of the Cx43-formed channel permeable dye Lucifer yellow, which is inhibited by the chemically unrelated Cx43-formed channel blockers carbenoxolone and heptanol, suggests a functional $\mathrm{Cx} 43$-formed channel within the inner membrane of subsarcolemmal mitochondria (MiroCasas et al., 2009; Soetkamp et al., 2014). The finding that the administration of the $\mathrm{Cx} 43 \mathrm{HC}$-specific peptide Gap19 as well as the genetic ablation of $\mathrm{Cx} 43$ reduce the potassium influx into the mitochondrial matrix further strengthens the hypothesis that $\mathrm{Cx} 43$-formed channels are present in the inner membrane of subsarcolemmal mitochondria (Miro-Casas et al., 2009; Soetkamp et al., 2014). Subsarcolemmal mitochondria are more responsive than interfibrillar mitochondria to fibroblast growth factor-2-triggered protection from calciuminduced permeability transition pore opening by a mitochondrial $\mathrm{Cx} 43$ channel-mediated pathway (Srisakuldee et al., 2014).

Apart from ion fluxes, both a pharmacological inhibition and a genetic ablation of $\mathrm{Cx} 43$ reduce mitochondrial complex 1-mediated oxygen consumption and ATP production, whereas complex 2-mediated respiration is not affected. Possibly, Cx43 interacts with proteins of complex 1 of the electron transport chain (Boengler et al., 2012; Denuc et al., 2016). Mitochondria are central to ROS formation and a relationship between $\mathrm{Cx} 43$ expression and ROS formation has been established (Matsuyama and Kawahara, 2011; Denuc et al., 2016), whereby $\mathrm{Cx} 43$-formed channel inhibition or Cx43 downregulation decrease the amounts of ROS formation.

It is not yet known whether all $\mathrm{Cx} 43$ phosphorylation sites described in section II.F are also phosphorylated in mitochondrial $\mathrm{Cx} 43$. However, phosphorylation of mitochondrial Cx43 is found at Ser-262 in rat subsarcolemmal mitochondria (Srisakuldee et al., 2014), whereas $\mathrm{Cx} 43$ phosphorylation at Ser-368 is detected in mouse, rat, and porcine subsarcolemmal mitochondria (Boengler et al., 2011; Srisakuldee et al., 2014; Shan et al., 2015). Phosphorylation of $\mathrm{Cx} 43$ is not necessarily achieved outside the mitochondria, since $\mathrm{PKC}$ is present in mitochondria and the stimulation of such mitochondrial PKC leads to $\mathrm{Cx} 43$ phosphorylation at Ser-262 and Ser-368 in rat subsarcolemmal mitochondria (Srisakuldee et al., 2014). Activation of mitochondrial $\mathrm{PKC}$ renders mitochondria more tolerant toward $\mathrm{Ca}^{2+}$ overload (Srisakuldee et al., 2014).

In addition to the well documented protective role of Cx43 in cardiac subsarcolemmal mitochondria, recent evidence indicates that mitochondrial $\mathrm{Cx} 43$ may also contribute to cardiac injury and cell death. Gadicherla et al. (2017) demonstrated that $\mathrm{Cx} 43$ forms functional hemichannels in cardiac subsarcolemmal mitochondria, which contribute to $\mathrm{Ca}^{2+}$ entry and trigger permeability transition and cell injury/cell death (Gadicherla et al., 2017). Compared to Gap26 and Gap19, RRNYRRNY peptide appeared to most active in inhibiting mitochondrial Cx43 hemichannel activity. The RRNYRRNY peptide also strongly reduced the infarct size in ex vivo cardiac ischemia-reperfusion studies (Gadicherla et al., 2017). Thus, Cx43 in cardiac subsarcolemmal mitochondria may protect in the context of ischemic preconditioning, but in the absence of preconditioning, it contributes to cardiac injury provoked by ischemia.

\section{B. Cx43 and Risk Factors of Cardiovascular Diseases}

1. Age and Sex. In the promotor region of the $\mathrm{Cx} 43$ gene, a series of half-palindromic estrogen response elements is present. In HeLa cells transfected with a luciferase-connexin43 promoter fusion construct, an upregulation of luciferase expression by estrogen occurs (Yu et al., 1994). Although Cx43 mRNA levels in ventricular tissue from neonatal male and female littermates are similar, the $\mathrm{Cx} 43 \mathrm{mRNA}$ levels are higher in adult female compared with age-matched male hearts (Rosenkranz-Weiss et al., 1994). Interestingly, phenylephrine treatment increases $\mathrm{Cx} 43$ expression only in female cardiomyocytes (Stauffer et al., 2011). Also on the protein level, expression of $\mathrm{Cx} 43$ is markedly lower in males of both normotensive and hypertensive rats compared with female rats (Knezl et al., 2008). The difference in $\mathrm{Cx} 43$ expression between males and females is predominantly seen at the level of GJs (Thomas et al., 2011). Multiple reports have documented that $\mathrm{Cx} 43$ protein expression declines with age in the hearts of hamsters (Chen and Jones, 2000), guinea pigs (Jones et al., 2004; Jones and Lancaster, 2015), mouse (Bonda et al., 2016), rats (Watanabe et al., 2004; Lancaster et al., 2011), and rabbits (Yan et al., 2013). Such reduced expression with age is associated with enhanced lateralization of the protein as observed in rat and rabbit hearts (Dhein and Hammerath, 2001; Fannin et al., 2014). Interestingly, $\mathrm{Cx} 43$ expression remains higher in aged (16 months) female compared with aged male rat hearts (Tribulova et al., 2005). Cx43 expression is reduced in the myocardium of postmenopausal or ovariectomized rats and such decline is attenuated by estrogen supplementation (Wang et al., $2015 b)$ or activation of protein kinase C (Lancaster et al., 2011). While in animal experiments $\mathrm{Cx} 43$ expression appears to be higher in female compared with male hearts, the $\mathrm{Cx} 43$ expression is higher in male than in female and higher in epicardial than in endocardial tissue samples from nondiseased human transplant donor hearts (Gaborit et al., 2010). Enhanced lateralization of $\mathrm{Cx} 43$, however, is detected in human tissue of aged patients (with atrial fibrillation), and this correlates with an increased incidence of atrial fibrillation (Kostin et al., 2002); similarly the age-dependent occurrence of arrhythmias in rat hearts is associated with lateralization of $\mathrm{Cx} 43$ (Fannin et al., 2014).

Recently, microRNAs (miR) have been found to regulate $\mathrm{Cx} 43$ expression within cardiomyocytes, 
including miR-1 (Xu et al., 2012) [which is higher expressed in males than in females, possibly explaining the sex difference in $\mathrm{Cx} 43$ expression (Stauffer et al., 2011)], miR-19a/b (Danielson et al., 2013), miR-23a (Wang et al., 2015b) (regulated via estrogen), and miR-130a (Osbourne et al., 2014) (also found in fibroblasts).

Finally, in aged mouse hearts, not only the amount of gap junctional $\mathrm{Cx} 43$ but also that of mitochondrial $\mathrm{Cx} 43$ is reduced (Boengler et al., 2007).

2. Hypertension and Hypertrophy. In spontaneously hypertensive rats, $\mathrm{Cx} 43$ protein expression either declines (Bačová et al., 2012; Benova et al., 2013; Zhang et al., 2014) or increases (Chen et al., 2007; Zhao et al., 2008; Radosinska et al., 2013). The differences between the aforementioned studies may be due to the degree of left ventricular hypertrophy associated with hypertension, because in porcine and human myocardium $\mathrm{Cx} 43$ expression increases in the compensated phase but gradually decreases with the progression/decompensation of hypertrophy (Formigli et al., 2003; Kostin et al., 2004).

In mice, pressure overload by transverse aortic constriction reduces the expression of $\mathrm{Cx} 43$ due to increased ubiquitination; this effect is blunted by knockdown of AMP kinase (Alesutan et al., 2015). When the ubiquitinase Wwp1 is globally overexpressed in mice, the highest induction of Wwp1 expression is observed in the heart associated with a $90 \%$ reduction in cardiac $\mathrm{Cx} 43$ protein expression, left ventricular hypertrophy, and the development of lethal ventricular arrhythmias (Basheer et al., 2015). Cardiomyocytespecific overexpression of calcineurin induces cardiomyocyte hypertrophy and downregulation of $\mathrm{Cx} 43$ expression and phosphorylation (Sun et al., 2015c). Myocardial Cx43 expression is also significantly decreased and redistributed after hypertrophy induced by prolonged isoproterenol treatment in rats in vivo. The decrease and redistribution of $\mathrm{Cx} 43$ is reduced after treatment with the ATP-dependent $\mathrm{K}^{+}$channel agonist nicorandil (Sun et al., 2015c). In contrast, rats with left ventricular hypertrophy secondary to transverse aortic constriction showed a decrease in miR-1 and an increase of $\mathrm{Cx} 43$ expression and phosphorylation at epitopes displacing $\mathrm{Cx} 43$ from the GJs (Curcio et al., 2013).

Interventions known to reduce hypertension and/or hypertrophy such as blockade or deletion of angiotensin II receptor I (Zhao et al., 2008; Yasuno et al., 2013), aldosterone antagonists (Qu et al., 2009), or renin inhibition (Zhang et al., 2014) prevent the changes in Cx43 expression, phosphorylation, or lateralization seen with hypertension and/or hypertrophy. Interestingly, melatonin also attenuates abnormal myocardial Cx43 distribution, upregulates $\mathrm{Cx} 43 \mathrm{mRNA}$ and protein expression, and maintains $\mathrm{Cx} 43$ phosphorylation forms in spontaneously hypertensive rats (Benova et al., 2013). With hypertension secondary to abdominal aortic constriction, $\mathrm{Cx} 43$ expression is reduced and $\mathrm{Cx} 43$ is displaced from the intercalated discs (Emdad et al., 2001), the effect being reversed by application of a H2S donors (Huang et al., 2012b).

The expression of mitochondrial $\mathrm{Cx} 43$ in hypertension and hypertrophy has not been studied up to now.

3. Diabetes and Hypercholesterolemia. In a prediabetes model in rats, $\mathrm{Cx} 43$ expression is unaltered and the fraction of $\mathrm{Cx} 43$ localized at the intercalated discs is increased (Axelsen et al., 2015). Cx43 mRNA and protein levels are either reduced (Lin et al., 2006a,b; Veeranki et al., 2016), unchanged (Nygren et al., 2007; Stables et al., 2014), or elevated (Howarth et al., 2008; Radosinska et al., 2013; Anna et al., 2014; Joshi et al., 2015) with diabetes. Enhanced lateralization of $\mathrm{Cx} 43$ is observed with diabetes in rat hearts with unaltered (Nygren et al., 2007) or increased expression of $\mathrm{Cx} 43$ (Radosinska et al., 2013; Anna et al., 2014; Joshi et al., 2015). The decreased amount of $\mathrm{Cx} 43$ in obese-diabetic mice is restored by moderate exercise (Veeranki et al., 2016) and the lateralization of $\mathrm{Cx} 43$ is normalized by omega-3-polyunsaturated fatty acid administration (Anna et al., 2014). With diabetes, overall (Radosinska et al., 2013) and tyrosine (Joshi et al., 2015) Cx43 phosphorylation is decreased, but increased $\mathrm{Cx} 43$ phosphorylation has also been described (Lin et al., 2006b; Anna et al., 2014; Palatinus and Gourdie, 2016).

In rabbits fed a high-cholesterol diet, the expression of $\mathrm{Cx} 43$ is increased in left atria (Lee et al., 2012), but is decreased in left ventricular tissue, where also the lateralization of $\mathrm{Cx} 43$ is enhanced (Lin et al., 2005). Such disturbed distribution of $\mathrm{Cx} 43$ is also found in rats fed a high-fat diet; however, in contrast to rabbits, the left ventricular expression of $\mathrm{Cx} 43$ remains unchanged, whereas the mitochondrial amount of $\mathrm{Cx} 43$ is decreased (Görbe et al., 2011).

4. Postmyocardial Infarction. Overall $\mathrm{Cx} 43$ expression decreases and gap junctional localization of $\mathrm{Cx} 43$ is disturbed, i.e., lateralized, postmyocardial infarction in the border and infarct zones of mouse (Lindsey et al., 2006; Jackson et al., 2008), rat (Matsushita et al., 1999; Savi et al., 2016), and dog (Peters et al., 1997) hearts. Wall stretch can influence $\mathrm{Cx} 43$ expression and localization either directly or indirectly via angiotensin II (Zhuang et al., 2000; Saffitz and Kleber, 2004; Hussain et al., 2010; Salameh et al., 2010). The Cx43 expression after myocardial infarction is preserved in matrixmetalloproteinase 7-deficient mice, indicating that Cx43 represents a matrix-metalloproteinase 7 substrate (Lindsey et al., 2006). The lateralization of $\mathrm{Cx} 43$ is limited when mice receive omega- 3 fatty acids before myocardial infarction (Baum et al., 2012). The transplantation of cardiac stem cells elevates $\mathrm{Cx} 43$ expression after myocardial infarction in rat hearts (Hou et al., 2015), and also the combined administration of hepatocyte growth factor and insulin-like growth factor improves $\mathrm{Cx} 43$ expression and intercellular coupling (Savi et al., 2016). The role of mitochondrial $\mathrm{Cx} 43$ expression 
postmyocardial infarction has not been studied so far. Further details on postmyocardial infarction $\mathrm{Cx} 43$ alterations in the context of their arrhythmogenic consequences is given in section VI.

5. Heart Failure. The $\mathrm{Cx} 43$ expression is reduced in a pacing-induced heart failure model in rabbits (Ai and Pogwizd, 2005). Here, the overexpression of $\mathrm{Cx} 43$ in cardiomyocytes from failing hearts to protein levels comparable to that of control cells improves cell coupling (Ai et al., 2010). Additionally, total $\mathrm{Cx} 43$ protein expression decreases in failing mice (Danielson et al., 2013), rat (Wang et al., 2012b; dos Santos et al., 2016), and dog (Akar et al., 2004) hearts. Moreover, a heterogeneous distribution of $\mathrm{Cx} 43$ at the plasma membrane is observed in patients with ischemic and/or dilated endstage heart failure (Dupont et al., 2001; Kostin et al., 2003) resulting in a proarrhythmic potential (Glukhov et al., 2012) (for further discussion see section VI). The analysis of the phosphorylation status of $\mathrm{Cx} 43$ shows either dephosphorylation (Akar et al., 2004; Ai and Pogwizd, 2005) or phosphorylation at Ser-255 (Sato et al., 2008). Dephosphorylation of $\mathrm{Cx} 43$ in pacinginduced heart failure in rabbits is associated with increased activation of $\mathrm{p} 21$-activated kinase 1 and subsequently protein phosphatase $2 \mathrm{~A}$. In isolated cardiomyocytes, $\mathrm{Cx} 43$ dephosphorylation is abolished by inhibition of protein phosphatase 2A (Ai et al., 2011). The induction of mitophagy and matrix metalloproteinase activity is suggested to contribute to the decreased expression of $\mathrm{Cx} 43$ in heart failure (Givvimani et al., 2014), and the inhibition of the proteasome increases Cx43 amounts in adriamycin-induced heart failure in rats (Chen et al., 2015a).

Data on the role of mitochondrial $\mathrm{Cx} 43$ in heart failure are limited. One study addressed the function of mitochondrial $\mathrm{Cx} 43$ in a model of doxorubicin cardiotoxicity in rat hearts and $\mathrm{H} 9 \mathrm{C} 2$ cells (Pecoraro et al., 2015). Doxorubicin induces mitochondrial translocation of $\mathrm{Cx} 43$ in a heat shock protein 90 -dependent manner, and blocking the $\mathrm{Cx} 43$ mitochondrial import increases ROS formation and mitochondrial calcium content and enhances depolarization of the mitochondrial membrane compared with doxorubin treatment alone. Thus, mitochondrial $\mathrm{Cx} 43$ appears to be important for the protection against doxorubin-induced cardiotoxicity. In a second study, the induction of dilated cardiomyopathy by furazolidone decreases the amount of $\mathrm{Cx} 43$ in both the myocardium and isolated mitochondria (Shan et al., 2015) and increased mitochondrial $\mathrm{Cx} 43$ is dephosphorylated at Ser-368. The functional importance of these mitochondrial changes remains unknown.

\section{Cx43 in Ischemia-Reperfusion Injury}

Cx43-formed HCs open upon metabolic inhibition (Kondo et al., 2000; Shintani-Ishida et al., 2007) or ischemia (Johansen et al., 2011), leading to release of $\operatorname{ATP}\left(\right.$ Clarke et al., 2009), changes in $\left[\mathrm{Ca}^{2+}\right]_{\mathrm{i}}$ and $\left[\mathrm{Na}^{+}\right]_{\mathrm{i}}$
(Li et al., 2001), and finally cell swelling and rupture of the sarcolemma (for review, see Garcia-Dorado et al., 2012). Accordingly, HC inhibition by $18-\alpha \mathrm{GA}$ or the connexin mimetic peptide Gap26 is protective in neonatal cardiomyocytes after simulated ischemia (Shintani-Ishida et al., 2007; Clarke et al., 2009). The use of the specific HC inhibitor Gap19 protects cardiomyocytes from volume overload, increases cell viability, and reduces infarct size after ischemia- reperfusion (Wang et al., 2013c).

The use of less HC-specific inhibitors such as Gap26/Gap27 also reduces myocardial infarction after ischemia-reperfusion (Hawat et al., 2010, 2012), and mice in which $\mathrm{Cx} 43$ was replaced by $\mathrm{Cx} 32$ have smaller infarcts than wild-type mice (Rodríguez-Sinovas et al., 2010). Although in $\mathrm{Cx} 43^{+/-}$mice myocardial infarction after ischemia-reperfusion is either unchanged (Schwanke et al., 2002; Heinzel et al., 2005; Sanchez et al., 2013) or reduced (Kanno et al., 2003), any further reduction of the $\mathrm{Cx} 43$ expression in conditional knockout mice results in smaller infarcts after ischemiareperfusion (Sanchez et al., 2013).

$\mathrm{Cx} 43$ phosphorylation and dephosphorylation during ischemia-reperfusion is closely associated with cellular ATP levels (Turner et al., 2004). In particular, ischemia or hypoxia induce dephosphorylation of $\mathrm{Cx} 43$ at Ser-365 (Solan et al., 2007) or at Ser-325/328/330 (Lampe et al., 2006), but also Cx43 phosphorylation at Ser-262, Ser-368, or Ser-373 (Ek-Vitorin et al., 2006; Dunn and Lampe, 2014; Smyth et al., 2014). Ser-365 is suggested to function as a "gatekeeper," since Ser-365 phosphorylation prevents Ser-368 phosphorylation (Solan et al., 2007) (see section II.F.4.b). The interaction between $\mathrm{Cx} 43$ and ZO-1 is regulated by phosphorylation at the Akt target site Ser-373, whereby the phosphorylation of $\mathrm{Cx} 43$ at Ser-373 disturbs the interaction between the two proteins, resulting in increased size of GJs and enhanced cell-cell communication (Dunn and Lampe, 2014). The mutation of Ser-373 to phosphorylationinsensitive Ala reduces the interaction between $\mathrm{Cx} 43$ and 14-3-3- proteins, stabilizing $\mathrm{Cx} 43$ at the plasma membrane and thereby avoiding $\mathrm{Cx} 43$ degradation (Smyth et al., 2014). During ischemia, a redistribution of $\mathrm{Cx} 43$ occurs from the intercalated disks to the lateral sides of the cardiomyocytes (Beardslee et al., 2000). Such lateralized $\mathrm{Cx} 43$ is mainly dephosphorylated, whereas phosphorylated $\mathrm{Cx} 43$ remains at the GJs (Severs et al., 2008). Recent data show that ischemia induces $\mathrm{Cx} 43$ ubiquitination at the intercalated disks and subsequent autophagic degradation of the protein requiring both AMP kinase (during ischemia) and Beclin 1 (during reperfusion) (Martins-Marques et al., 2015b,c). Ubiquitination of $\mathrm{Cx} 43$ is decreased by the mutation of the phosphorylation site Ser-373 to Ala (Smyth et al., 2014). Also matrix-metalloproteinase 9 appears to be involved in the hypoxia-induced degradation of $\mathrm{Cx} 43$ in H9c2 cells (Wu et al., 2013). 
In $\mathrm{H} 9 \mathrm{C} 2$ cells subjected to 12 -hour hypoxia, the phosphorylation of mitochondrial $\mathrm{Cx} 43$ is increased; however, no specific phosphorylation sites have been tested (Waza et al., 2014). After a shorter phase of ischemia (30-minute ischemia, 5-minute reperfusion), an overall dephosphorylation of $\mathrm{Cx} 43$ is observed in rat myocardial mitochondria (Görbe et al., 2011). Also, in porcine myocardial mitochondria, ischemia induces a dephosphorylation at the PKC-target site of $\mathrm{Cx} 43$, namely Ser-368 (Totzeck et al., 2008).

Several pharmacological interventions are known to reduce infarct size after ischemia-reperfusion and some of them impact on the phosphorylation status of $\mathrm{Cx} 43$. Metabolic inhibition significantly reduces dye coupling of cardiomyocytes that is prevented by pretreatment with estradiol; the beneficial effect of estradiol is abolished by tamoxifen, a potent estrogen receptor antagonist. Double immunofluorescence microscopy shows that metabolic inhibition induces the accumulation of nonphosphorylated $\mathrm{Cx} 43$ at GJs and that this is prevented by estradiol pretreatment. Inhibition of protein kinase $\mathrm{C}$ with chelerythrine blocks the estrogeninduced increase of phosphorylated $\mathrm{Cx} 43$ (Chung et al., 2004).

In canine hearts, administration of $17 \beta$-estradiol decreases myocardial infarction, and this effect is associated with a decrease in the overall phosphorylation of Cx43 (Lee et al., 2004). Also, inhibition of p38 MAPK induces phosphorylation of $\mathrm{Cx} 43$ at Ser-368 (Surinkaew et al., 2013). The sphingosine-1 phosphate (S1P) constituent of high-density lipoproteins limits cell death by ischemia-reperfusion, and this is accompanied with an increased Cx43 phosphorylation at Ser-368. A causal role for this phosphorylation site in cardioprotection by sphingosine-1 phosphate is shown in mice, in which Ser-368 of $\mathrm{Cx} 43$ is mutated to Ala; here, the infarct size reduction by sphingosine-1 phosphate is lost (Morel et al., 2016). Enhanced phosphorylation of $\mathrm{Cx} 43$ (total and mitochondrial) at Ser-262 and Ser-368 is achieved in isolated rat hearts by diazoxide or fibroblast growth factor 2 (FGF-2) (Srisakuldee et al., 2009, 2014). In neonatal cardiomyocytes, the overexpression of Cx43, in which Ser-262 is mutated to alanine, results in augmented cell death after simulated ischemia (Srisakuldee et al., 2009).

\section{Cx43 and Cardioprotection}

The phenomena of endogenous conditioning describe the infarct size reduction after sustained ischemiareperfusion by brief nonlethal periods of ischemiareperfusion, which are performed either before (pre-), during (per-), or after (post-) the sustained phase of ischemia followed by reperfusion. The protection by such endogenous conditioning is often attenuated or lost in the presence of cardiovascular risk factors or diseases (Ferdinandy et al., 2014), where total and mitochondrial $\mathrm{Cx} 43$ expression, localization, and/or phosphorylation is altered. Ischemic preconditioning (IPC) suppresses chemical coupling of cardiomyocytes via GJs during ischemia. By using a protocol of simulated ischemiareperfusion in vitro, only wild-type cardiomyocytes are protected by IPC, whereas cardiomyocytes isolated from $\mathrm{Cx} 43^{+/-}$animals show no preservation of cell viability (Li et al., 2004a). In $\mathrm{Cx} 43^{+/-}$animals, the infarct size reduction by IPC is abolished (Schwanke et al., 2002), whereas noninduced conditional $\mathrm{Cx} 43$ knockout mice (also having a $50 \%$ reduction of $\mathrm{Cx} 43$ ) demonstrate cardioprotection by IPC (Sanchez et al., 2013). The further reduction of the $\mathrm{Cx} 43$ content in these mice; however, abolishes IPC-induced cardioprotection (Sanchez et al., 2013). In addition, in mice, in which $\mathrm{Cx} 43$ is replaced by $\mathrm{Cx} 32$, IPC is no longer effective (RodríguezSinovas et al., 2010). When Cx43-formed channels are inhibited by heptanol during the preconditioning cycles of ischemia and reperfusion infarct size reduction by IPC is lost ( $\mathrm{Li}$ et al., 2002).

The analysis of the phosphorylation status of $\mathrm{Cx} 43$ reveals that IPC prevents the ischemia-induced dephosphorylation of $\mathrm{Cx} 43$ in rat hearts in vitro (Jain et al., 2003) and in vivo (Hatanaka et al., 2004) or in pig hearts in vivo (Ahlquist et al., 1989; Totzeck et al., 2008); in one study, however, IPC had no impact on Cx43 phosphorylation (Mühlfeld et al., 2010). Preserved Cx43 phosphorylation is in part due to the increased activity of PKC, since knockout of PKC not only abrogates the protection but also the maintained $\mathrm{Cx} 43$ phosphorylation by IPC (Hund et al., 2007).

IPC not only affects gap junctional, but also mitochondrial $\mathrm{Cx} 43$. The amount of the protein within mitochondria is increased upon IPC in isolated mouse (Sun et al., 2015b) and rat hearts (Boengler et al., 2005; Soetkamp et al., 2014) and pig hearts in vivo (Boengler et al., 2005). In bone marrow stem cells, the overexpression of specifically mitochondrial $\mathrm{Cx} 43$ is sufficient to confer cytoprotection (Lu et al., 2010), pointing to the important role of mitochondrial $\mathrm{Cx} 43$ in cell survival.

Pharmacological preconditioning with diazoxide augments mitochondrial $\mathrm{Cx} 43$ in a model of hypothermia (Yang et al., 2011). When the mitochondrial import of Cx43 is blocked by the use of the Hsp90-inhibitor geldanamycin, the infarct size reduction by diazoxide is lost. Since in this model the gap junctional content of Cx43 remains unaffected, the loss of cardioprotection by diazoxide is associated with the reduced content of mitochondrial $\mathrm{Cx} 43$. In addition to $\mathrm{Cx} 43$, geldanamycin also inhibits the mitochondrial import of $\mathrm{PKC}_{\varepsilon}$, which may phosphorylate mitochondrial $\mathrm{Cx} 43$ (Budas et al., 2010). The analysis of the mechanisms by which mitochondrial $\mathrm{Cx} 43$ contributes to diazoxide-induced cardioprotection demonstrates that the generation of reactive oxygen species, which, if present in low amounts, function as trigger molecules of preconditioning, is reduced in cardiomyocytes isolated from 
Cx43-deficient mice (Heinzel et al., 2005). Furthermore, IPC induces $S$-nitrosation, an important posttranslational modification in cardioprotection (Lin et al., 2009), predominantly of subsarcolemmal mitochondrial proteins (Sun et al., 2015b). One protein with enhanced amounts of $S$-nitrosation after IPC is mitochondrial $\mathrm{Cx} 43$, and the $S$-nitrosation of mitochondrial $\mathrm{Cx} 43$ enhances the permeability of subsarcolemmal mitochondria toward potassium ions and increases reactive oxygen species formation (Soetkamp et al., 2014). Subsarcolemmal mitochondria isolated from wild-type mice show increased respiration when the isolated mitochondria undergo IPC; however, in mice in which $\mathrm{Cx} 43$ is replaced by Cx32, such preserved oxygen consumption is lost (Ruiz-Meana et al., 2014). In addition, mitochondrial Cx43 may contribute to cardioprotection via its effect on permeability transition pore opening (see above), because the inhibition of transition pore opening reduces myocardial infarction (Ong et al., 2015).

In remote ischemic preconditioning, where the short periods of ischemia-reperfusion occur in organs other than the heart, sustained $\mathrm{Cx} 43$ expression and phosphorylation is observed in hearts after ischemiareperfusion (Brandenburger et al., 2014).

Ischemic postconditioning induces $\mathrm{Cx} 43$ phosphorylation in rat hearts in vivo (Wu et al., 2012; He et al., 2015), but also in mitochondria isolated from mice hearts that were subjected to a postconditioning protocol (Boengler et al., 2011). However, one study demonstrated reduced phosphorylation of mitochondrial Cx43 after postconditioning compared with ischemiareperfusion alone (Penna et al., 2009). Nevertheless, altered $\mathrm{Cx} 43$ phosphorylation is not important for the cardioprotection by postconditioning, since $\mathrm{Cx} 43^{+/-}$ mice still have smaller infarcts than wild-type mice upon a postconditioning stimulus (Heusch et al., 2006).

Pharmacological cardioprotection by induction of HO-1 protects the rat heart against postischemic dysfunction and arrhythmias by a $\mathrm{Cx} 43$-dependent mechanism (Lakkisto et al., 2009).

Taken together, therapeutic activation of $\mathrm{Cx} 43$ expression/function may provide a novel tool for cardioprotection in ischemic heart disease.

\section{Connexins and Arrhythmias}

The heart is a rhythmically beating organ in which synchronized contraction is achieved by the organized propagation of action potentials between cells. The normal heart rhythm requires a pacemaker region, the sinus node, from which the electrical activity is transferred to the atrium, then the atrioventricular node, the bundle of His, the Tawara branches, and (via the Purkinje fibers) the working ventricular myocardium. Within the ventricular conduction system, the action potential propagates with a velocity of about
$0.8-1.0 \mathrm{~m} / \mathrm{s}$, whereas the conduction velocity within the working myocardium is about $0.4 \mathrm{~m} / \mathrm{s}$ along the fibers and $0.2 \mathrm{~m} / \mathrm{s}$ transverse to the fiber axis. The regional and directional differences in conduction velocity, termed anisotropy, are basic characteristics of cardiac tissue and can be either uniform or nonuniform (Spach and Dolber, 1990). Successful and sustained coupling requires $>38$ GJ channels per cell, whereas conduction block occurs if less than 13 are present. In addition to supporting the role of GJs in cardiac cell coupling, the classic experiments of Weingart and Maurer (1988) excluded the possibility that action potentials can be transferred via ephaptic coupling (electrical field effects) or local ionic interactions at the level of closely appositioned membranes. However, under certain pathophysiological conditions, such phenomena may contribute to conduction between cardiomyocytes (Veeraraghavan et al., 2015; George et al., 2016; George and Poelzing, 2016).

The different anisotropic and conductive characteristics of different cardiac tissues derive, in part, from differences in the connexin isoforms expressed and the distributions of GJs (Saffitz et al., 1992, 1994; Davis et al., 1994; Saffitz et al., 1994).

Targeted ablation of several connexins in mice has demonstrated their importance for electrical coupling in various cardiovascular tissues and has shown that their absence can lead to various arrhythmias or sudden death (Kirchhoff et al., 1998; Simon et al., 1998; Gutstein et al., 2001a,b; Kreuzberg et al., 2006). Such studies also revealed that a reduction in $\mathrm{Cx} 43$ expression of about $50 \%$ is required before conduction velocity is reduced (Guerrero et al., 1997; Thomas et al., 1998; Morley et al., 1999; Morley et al., 2000). However, the degree of conduction slowing appears to be variable, with some studies demonstrating no slowing and others reporting $23 \%-44 \%$ reduced conduction velocity (reviewed in Tse and Yeo, 2015). This apparent disparity has been linked to the ionic composition of the extracellular fluid and ephaptic coupling (George and Poelzing, 2016). In line with the findings from $\mathrm{Cx} 43$ knockdown studies, slowing of the conduction velocity from 0.23 to $0.16 \mathrm{~m} / \mathrm{s}$ by means of a low concentration of heptanol $(0.05 \mathrm{mM})$ was found to be sufficient to increase the inducibility of ventricular tachycardia in isolated mouse hearts. This occurred without affecting the effective refractory period, so that cardiac excitation wavelength (the product of conduction velocity and effective refractory period) was shortened (Tse et al., 2016b).

For propagation along the bundle of His, the Tawara branches, and the Purkinje fibers, it is important that the conduction bundle does not communicate with the surrounding cardiac tissue, because otherwise the activation would start at the valvular level. This isolation is achieved by differential connexin expression: the conduction system communicates mainly via 
Cx40-containing GJ channels, whereas the working myocardium communicates via $\mathrm{Cx} 43$ channels. A specific biophysical problem occurs at the interface between these systems at the endings of the Purkinje fibers where the small Purkinje fiber has to activate a large surrounding tissue (known as the "current source/ sink problem"). Elicitation of an action potential requires a depolarizing current from an adjacent, already activated cell. The activated cell represents a current source and the quiescent cell a current sink. The voltage difference between these two cells is the driving force for this current that flows via GJ channels (and, to some extent, via the extracellular space). The quantity of current transferred is determined by geometric properties of the tissue. Thus, if a tiny cluster of activated cardiomyocytes (i.e., a small source) needs to activate a large surrounding region (a large sink), current flows radially from the activated cluster to many nonactivated sites, so that the current is distributed to a large number of cells. As a consequence, the total charge transferred to each of these neighboring cells may be too low to activate these cells, thereby causing conduction failure (Rohr et al., 1997; Rohr, 2004, 2012; Lee and Pogwizd, 2006). If GJ conductance is reduced, it may prevent conduction block, since less current is lost to the adjacent cells. Thus, a small source can activate only a very limited number of neighboring cells. A similar situation is present in the sinus node, where the weak conduction between a tiny current source (sinus node) and a large sink (atrium) is achieved by a limited expression of GJs localized in in interdigitating finger-like zones extending from the sinus node into the atrium (Joyner and van Capelle, 1986; Boyett et al., 2006). In hypokalemia, shortening of ventricular effective refractory period without changes in conduction velocity has been observed. Interestingly $0.1 \mathrm{mM}$ heptanol restored the effective refractory period and acted anti-arrhythmically, which may support the hypothesis that also GJ reduction may prevent from arrhythmia in certain situations (Tse et al., 2016a).

A general mathematical formulation describes the ratio of charge produced/charge consumed as the safety factor, which equals $Q_{c}+Q_{\text {out }} / Q_{\text {in }}$ (Shaw and Rudy, 1997a,b). If coupling is reduced gradually, the safety factor first is enhanced as a result of the smaller space constant. However, if very low levels of coupling are reached, SF decreases until $\mathrm{SF}<1$ and conduction failure occurs (Shaw and Rudy, 1997a,b). A high curvature of the propagation wave front typically causes such source-sink-problems. Moreover, typical situations with source-sink problems are the endings of Purkinje fibers, areas in which the activation front passes a narrow isthmus, encircles obstacles, or at the border between ischemic and nonischemic tissue, where the ischemic nonexcitable tissue acts as a large current sink. The relationship between cell surface $\left(A_{m}\right.$, which is linearly related to cell capacitance) and GJ conductance $\left(\mathrm{g}_{\mathrm{GJ}}\right)$ is also important. An increased cell diameter may accelerate longitudinal propagation velocity $\theta_{\mathrm{L}}$, if $\mathrm{g}_{\mathrm{GJ}}$ is enhanced proportionally to the cellular membrane surface $A_{m}$. In contrast, in the case of constant $\mathrm{g}_{\mathrm{GJ}}$, increased diameter leads to reduced $\theta_{\mathrm{L}}$. Consequently, the $\mathrm{g}_{\mathrm{GJ}} / \mathrm{A}_{\mathrm{m}}$ ratio controls whether alterations in cell size will enhance or reduce $\theta_{\mathrm{L}}$ (Seidel et al., 2010).

Taken together, altered arrhythmogenicity in relation to GJs may be explained by alterations in conduction velocity, in particular conduction failure either due to complete block of conduction or to a source-sink mismatch, by alterations in conduction pathways, by changes in refractory periods, e.g., due to enhanced or reduced current flow via GJs to adjacent cells. A comprehensive overview of the biophysical basis of arrhythmia and normal conduction is presented in Dhein et al. (2014). Below we further discuss the role of GJs in ventricular and atrial fibrillation.

\section{A. Gap Junctions and Ventricular Fibrillation}

Ventricular fibrillation (VF) is among the most dangerous arrhythmias. It often occurs after acute or chronic myocardial infarction or in patients with heart failure. Typically VF is driven by a spiral wave rotator that encircles a nonexcitable obstacle like a scar (Krinsky, 1978) or a permanently depolarized area (Karagueuzian et al., 1998), forming an abnormal circuit like the "leading circle" identified in the atrium (Allessie et al., 1977). According to these theories, ventricular fibrillation is driven either by a single rapidly drifting rotor or by a stationary rotor (Samie and Jalife, 2001). Such spiral waves are promoted by reduced excitability or reduced conduction velocity (Mandapati et al., 1998). The diameter of a rotator may be very small (hundreds of micrometers). In infarcted hearts, the infarct zone often is not a homogenous zone, but contains patchy fibrosis; the likelihood of VF induction is positively related to the patchiness of this area (Karagueuzian et al., 1986).

Structurally, VF is favored by nonuniform anisotropy, e.g., small islets of connective tissue, fibroblasts, or scars. These structures lead to 1) a delay in conduction (Rohr et al., 1997) and 2) inhomogeneity in action potential duration (APD) (Lesh et al., 1989; Müller and Dhein, 1993) resulting in shortened APD at sites where a cell encounters a large nonactivated area (current sink) and where conduction delay may prolong the time during which activated cells lose current to adjacent sites (Müller and Dhein, 1993; Spach and Josephson, 1994; Gottwald et al., 1998). The interaction of cardiomyocytes and nonexcitable fibroblasts in such areas is a topic of substantial ongoing investigation. Both of these cell types can be coupled via GJs typically consisting of $\mathrm{Cx} 45$ or $\mathrm{Cx} 43$ as determined from immunostainings or from their single channel conductances (Rook et al., 1989; Camelliti et al., 2004), although the 
fibroblast is electrically inactive. Such heterocellular communication may explain the conduction of action potentials for up to $300 \mu \mathrm{m}$ through connective tissue/ fibroblast areas (Rohr, 2004; Brown et al., 2015), but with significantly slowed conduction velocity (Nguyen et al., 2014). In aged hearts, the degree of fibrosis is enhanced, and as a consequence, the activation pattern shows more irregularities (Dhein and Hammerath, 2001). Similarly, enhanced microfibrosis is also found in failing hearts (Glukhov et al., 2012). Stem cells seem to be able to establish GJ-mediated intercellular communication with cardiomyocytes and may similarly generate a risk for arrhythmia (Smit and Coronel, 2014). Most recently it was reported that even macrophages can form Cx43-based GJs with cardiomyocytes, thereby assisting in AV nodal conduction but also affecting repolarization (Hulsmans et al., 2017).

What is the role of GJs in conditions leading to ventricular fibrillation? In acute ischemia (see Fig. 4), intracellular $\mathrm{pH}$ drops and ATP is lost; consequently, the activity of membrane pumps like the $\mathrm{Na}^{+} / \mathrm{K}^{+}$pump and the $\mathrm{Na}^{+} / \mathrm{Ca}^{2+}$ exchanger are reduced, resulting in increased intracellular $\mathrm{Na}^{+}$and $\mathrm{Ca}^{2+}$ concentrations and membrane depolarization. ATP loss and accumulation of $\mathrm{Na}^{+}, \mathrm{H}^{+}$, and $\mathrm{Ca}^{2+}$ inside the cells reduce GJ conductance. Intracellular acidosis activates the $\mathrm{Na}^{+} / \mathrm{H}^{+}$exchanger and the $\mathrm{HCO}_{3}^{-} / \mathrm{Na}^{+}$symporter, thereby enhancing the intracellular rise in $\mathrm{Na}^{+}$. In consequence of reduced GJ conductance and $\mathrm{Na}^{+}$current availability, conduction is delayed or even blocked. In addition, lysophosphoglycerides, long chain acylcarnitines, and arachidonic acid metabolites accumulate and further reduce GJ intercellular communication $(\mathrm{Wu}$ et al., 1993). More recent investigations also found that myocardial $\mathrm{Cx} 43$ becomes dephosphorylated after 30 minutes of regional ischemia and is removed from the cell membrane accompanied by conduction slowing (Jozwiak and Dhein, 2008). Conduction slowing may allow the activation wave to circle around a nonexcitable zone, reaching its starting point when repolarization has just finished. This condition is a typical condition to establish for reentry circuits.

In acute ischemia, there are two peaks of $\mathrm{VF}$ incidence (Smith et al., 1995): the first (type Ia) occurs within 5 minutes after onset of ischemia, the second (type Ib) occurs after about 20-30 minutes. The early peak is often related to factors such as catecholamine release, depolarization, and mechanical factors together with shortened action potentials. The second peak (Ib) has been linked to GJ uncoupling, since increased tissue impedance and conduction slowing were observed after 12-15 minute of ischemia (Dekker et al., 1996; Müller et al., 1997b; de Groot et al., 2001). This uncoupling is caused by ATP depletion, intracellular acidosis, accumulation of acylcarnitines, dephosphorylation of $\mathrm{Cx} 43$ (especially at Ser-368) (Lampe et al., 2006; Jozwiak and Dhein, 2008), intracellular
$\mathrm{Na}^{+}$and $\mathrm{Ca}^{2+}$ overload due failure of the $\mathrm{Na}^{+} / \mathrm{K}^{+}-$ ATPase, and $\mathrm{Na}^{+} / \mathrm{Ca}^{2+}$ exchanger (Burt, 1987; Fluri et al., 1990; Massey et al., 1992; Morley et al., 1996; Hofgaard et al., 2008). Such ischemic uncoupling can be antagonized by antiarrhythmic peptides like AAP10 (Müller et al., 1997b; Dhein et al., 1994; Jozwiak and Dhein, 2008) or its derivative rotigaptide (Kjølbye et al., 2003; Xing et al., 2003). AAP10 has been shown to maintain the degree of phosphorylation of $\mathrm{Cx} 43$ in a PKC-dependent manner and to preserve the localization of $\mathrm{Cx} 43$ at the cell poles (Jozwiak and Dhein, 2008). In contrast, ischemia leads to a reduction in membraneassociated $\mathrm{Cx} 43$ and to a loss of polar $\mathrm{Cx} 43$. The inducibility of ventricular fibrillation may be regulated by a domain within the $\mathrm{Cx} 43 \mathrm{CT}$ region (Maass et al., 2009).

Ischemic uncoupling is typically accompanied by depolarization. Reduced I(Na) availability enhances dispersion of the action potential duration (APD), since local differences in APD causing voltage gradients can no longer be neutralized by GJ currents. Dispersion and conduction slowing (that occur in some cases) and nonexcitable areas (e.g., depolarized areas) are factors known to initiate reentrant arrhythmias (Janse, 1986; Janse et al., 1986; Coronel et al., 1989; Opthof et al., 1991; Wit and Janse, 1992; Spach and Josephson, 1994; el-Sherif et al., 1996). The voltage gradients between ischemic and nonischemic areas may also generate a flow of injury current that may be strong enough to depolarize surviving Purkinje strands (Janse et al., 1980).

Chronic infarction is characterized by necrosis of cardiomyocytes and replacement by connective tissue; it may produce electrically silent zones that interdigitate among normal cardiac tissue. It produces local inhomogeneity and conduction, slowing, or even conduction failure. The loss of contractile force reduces ejection fraction, causing left ventricular dilatation and increased wall tension with mechanical stretch.

As introduced in section $V, \mathrm{Cx} 43$ expression is reduced in chronic infarction (Peters, 1995; Chen et al., 2010), resulting in reduced GJ communication and consequent conduction slowing (Yao et al., 2003). Some investigators have also observed increased abundance of $\mathrm{Cx} 43$ at the lateral side of the cardiomyocytes (Kieken et al., 2009), which (if functional) may result in enhanced transverse conduction and reduced anisotropy. The increased $\mathrm{Cx} 43$ expression may be regulated by angiotensin II, endothelin, or enhanced mechanical stretch (especially if cardiac function is compromised and cells become hypertrophic). Interactions between cardiomyocytes and fibroblasts may also be important in chronic infarction. Cocultures of both cell types (separated by a semipermeable membrane) revealed that fibroblasts release a soluble factor that inhibits $\beta$-adrenoreceptordependent increases in $\mathrm{Cx} 43$ expression (Salameh et al., 2013). If the infarcted area exceeds a certain size, heart 
failure occurs (perhaps associated with ischemic cardiomyopathy, see below).

Alterations in $\mathrm{Cx} 43$ expression and GJs are also found in heart failure, potentially contributing to associated arrhythmias. Heart failure may be characterized as compensated heart failure, hypertrophy, and decompensated heart failure and classified by four levels (NYHA I-IV), with the most severe level (IV) corresponding to patients who suffer from dyspnea even when lying in bed. The typical therapeutic option for NYHA IV patients is cardiac transplantation. "Heart failure specimens" for many studies derive from the explanted hearts of these patients; studies of gene and protein expression and regulation may be confounded by the many drugs that are given to these patients. Heart failure may also be distinguished according to pathophysiological considerations including 1) ischemic cardiomyopathy (ICM), 2) dilated cardiomyopathy (DCM), and 3) hypertrophic cardiomyopathy. Salameh et al. (2009) showed that polar and lateral $\mathrm{Cx} 43$ are increased in hypertrophic cardiomyopathy, but the polar fraction is reduced and the lateral fraction is nearly unchanged in cardiac biopsies from patients with dilated cardiomyopathy (NYHA II-III) (Salameh et al., 2009). These authors observed increased $\mathrm{Cx} 43 \mathrm{mRNA}$ and protein expression in hypertrophic cardiomyopathy patients and decreased $\mathrm{Cx} 43 \mathrm{mRNA}$ and $\mathrm{Cx} 43$ protein in DCM patients. The diminished $\mathrm{Cx} 43$ levels observed in DCM patients are accompanied by downregulated ZO-1 protein (Kostin, 2007). ZO-1 is a scaffolding protein for $\mathrm{Cx} 43$, and reductions of $\mathrm{Cx} 43$ may be result from its degradation after displacement from ZO-1 after phosphorylation by c-Src (Sovari et al., 2011; Rutledge et al., 2014). The regulators of altered $\mathrm{Cx} 43$ expression in heart failure include angiotensin II, endothelin, bFGF, catecholamines, and TNF- $\alpha$ (reviewed by Dhein, 2004).

In many cases, the pathogenic alterations of GJs may not be uniform across the ventricle. Reduced $\mathrm{Cx} 43$ expression was observed in samples obtained from human patients suffering from ICM, DCM, or inflammatory cardiomyopathies; but, interestingly, especially in the ICM patient samples, both areas with clearly reduced $\mathrm{Cx} 43$ expression and areas with nearly normal Cx43 expression and distribution were identified (Kostin et al., 2003). Thus, Kostin et al. (2003) concluded that "focal disorganization of GJ distribution and downregulation of $\mathrm{Cx} 43$ are typical features of myocardial remodeling that may play an important role in the development of an arrhythmogenic substrate in human cardiomyopathies."

It is apparent that local disorganizations of ventricular GJs may be more important for cardiac pathophysiology than overall changes in expression (Kitamura et al., 2002). Such a focal disorganization in the communicating GJ network together with fibrosis and changes in the cellular geometry (due to hypertrophic processes) may cause effects on the current source-sink relationships that can dramatically affect overall conduction and local propagation patterns (Spach and Dolber, 1990; Rohr et al., 1997; Spach et al., 2000; Rohr, 2004, 2012; Lee and Pogwizd, 2006; Seidel et al., 2010). Thus, the network consisting of cardiomyocytes with their specific shape, irregularities in $\mathrm{Cx} 43$ expression, changes in subcellular $\mathrm{Cx} 43$ distribution, microfibrosis, nonexcitable cells (like fibroblasts) can form the arrhythmogenic substrate.

Two factors affecting conduction are consistently observed in chronic heart disease: 1) the degree of fibrosis is enhanced, which increases nonuniformity, and 2) the expression/distribution of GJ proteins is altered. Often the abundance of connexins at the lateral side of the cardiomyocytes is increased relative to that at the ends. Increased coupling by these lateral junctions could increase transverse conduction (Seidel et al., 2010). Hypertrophy associated changes in cell size and geometry will also alter anisotropy. Altered ratios between longitudinal and transverse conduction have been described in ventricular preparations, atrial tissue, and computer simulations (Spach et al., 2000; Koura et al., 2002; Hubbard et al., 2007) (Dhein and Hammerath, 2001; Seidel et al., 2010; Dhein et al., 2011). These effects may be modulated by alterations of the extracellular space (Cabo and Boyden, 2009).

\section{B. Gap Junctions and Atrial Fibrillation}

Atrial fibrillation (AF) is the most common cardiac arrhythmia. It is characterized by a rapid and irregular electrical activation and the loss of atrial muscle contractility. The pathogenesis of AF involves initiating triggers (often rapidly firing ectopic foci located inside the pulmonary veins) and an abnormal atrial tissue substrate that maintains the arrhythmia (Allessie et al., 2001; Iwasaki et al., 2011). The tissue substrate is determined in large part by the abundance and distribution of intercellular channels contained within atrial GJs (Spach and Starmer, 1995).

Atrial GJs are comprised of two different subunit proteins, $\mathrm{Cx} 40$ and $\mathrm{Cx} 43$. They are each abundantly expressed by atrial myocytes with similar abundances and highly overlapping distributions (Lin et al., 2010; Gemel et al., 2014a). Together, $\mathrm{Cx} 40$ and $\mathrm{Cx} 43$ determine the properties of intercellular conduction within this tissue (Saffitz et al., 1994; Lin et al., 2010). A number of studies have investigated the possible role for GJs and connexins in atrial fibrillation in animal models or in human patients (reviewed by Dhein, 2006b; Duffy and Wit, 2008). Likely reflecting the heterogeneity of this disorder (idiopathic versus secondary and paroxysymal versus persistent versus permanent), a wide diversity of different results have been obtained including increases, decreases, or lack of changes. Various alterations of both $\mathrm{Cx} 40$ and $\mathrm{Cx} 43$ have been observed in animals and human patients with AF (reviewed in Kato et al., 2012). Below, we 
further discuss alterations of these connexins at the level of messenger RNA, protein expression, posttranslational modifications, distribution, and genetic abnormalities or mutations.

1. Connexin mRNAs. Taken together, there is little consistent evidence to suggest significant changes in Cx43 expression or levels. Cx40 mRNA levels are reduced in some mice with cardiac targeted defects in the heart (Sawaya et al., 2006), but other animal studies provide little evidence for alterations of $\mathrm{Cx} 40 \mathrm{mRNA}$ (van der Velden et al., 2000). Cx40 mRNA may be reduced in some patients with valvular heart disease or heart failure associated with $\mathrm{AF}$ (Nao et al., 2003; Gaborit et al., 2005), but other studies have shown no changes. Moreover, examination of the expression profile data available in the GEO data sets (http://www. ncbi.nlm.nih.gov/projects/geo/gds) shows no consistent difference in $\mathrm{Cx} 40 \mathrm{mRNA}$ (or that of other connexins) between $\mathrm{AF}$ and control patients.

2. Connexin Protein Levels. Most reports have observed no major changes in $\mathrm{Cx} 43$ abundance detectable by immunoblotting (Polontchouk et al., 2001). Perhaps because good anti-Cx40 reagents had not been widely available until recently, there is little published data regarding $\mathrm{Cx} 40$ levels detected by immunoblotting. Immunohistochemistry has been used in a number of studies to estimate levels of immunoreactive $\mathrm{Cx} 40$ or $\mathrm{Cx} 43$. Some studies have found decreases in $\mathrm{Cx} 40$ and/or Cx43 (Kostin et al., 2002), but the most consistent finding is a decrease in the $\mathrm{Cx} 40 / \mathrm{Cx} 43$ ratio (implying a greater reduction of $\mathrm{Cx} 40$ than $\mathrm{Cx} 43$ ) (van der Velden et al., 2000; Kanagaratnam et al., 2004; Wilhelm et al., 2006). Since mediators like angiotensin II (which can be released in AF or upon stretch) can upregulate $\mathrm{Cx} 43$ expression and other factors like ischemia can reduce $\mathrm{Cx} 43$ expression, the degree and the type of alteration of connexin expression (increase or decrease) is known to be affected by the comorbidity of the patient (see e.g., Wetzel et al., 2005). Thus it is necessary to differentiate at least between lone $\mathrm{AF}$, mitral valve disease related $\mathrm{AF}$, and $\mathrm{AF}$ in coronary heart disease.

3. Posttranslational Modifications of Connexins. As noted above, the major cardiac connexins are all phosphoproteins, and different phosphorylation events can regulate many aspects of GJ assembly, function, and degradation. c-Jun N-terminal kinase (JNK) activation is implicated in cardiovascular diseases and aging, which are linked to enhanced propensity to atrial fibrillation (AF). In a rabbit model, Yan et al. (2013) showed that JNK activation contributed to $\mathrm{Cx} 43$ reductions that promote development of AF (Yan et al., 2013).

4. Connexin Distribution. The other frequent finding in both animal models and human AF is heterogeneity of connexin staining (i.e., some areas show intense GJ immunostaining, whereas others show weak or no staining). When both connexins have been studied in the same samples, the heterogeneity is often more severe for Cx40 (van der Velden et al., 1998; Kanagaratnam et al., 2004).

5. Genetic Abnormalities of Cx40 Linked to Atrial Fibrillation. Two groups of studies suggest AF-associated genetic abnormalities of $\mathrm{Cx} 40$. The Cx40 (GJA5) gene contains two closely linked polymorphisms in the promoter region, $-44(\mathrm{G} \rightarrow \mathrm{A})$ and +71 $(\mathrm{A} \rightarrow \mathrm{G})$. One haplotype $(-44 \mathrm{~A} / \mathrm{+}+\mathrm{G})$ is relatively rare, and it has been associated with familial atrial standstill (Groenewegen et al., 2003) and atrial fibrillation (Hauer et al., 2006). This polymorphic variant has reduced promoter activity in in vitro reporter gene experiments (Firouzi et al., 2006), suggesting that it might lead to reduced $\mathrm{Cx} 40$ expression in people who carry this polymorphism. Coding region variants of $\mathrm{Cx} 40$ may also sometimes contribute to AF. Gollob et al. (2006) examined the sequence of the $\mathrm{Cx} 40$ coding region in atrial tissue obtained from 15 patients with lone $\mathrm{AF}$ (Gollob et al., 2006). They identified four patients with mutations: three patients apparently had somatic mutations and the fourth patient had a germline mutation. Subsequent reports have identified individuals or families with additional germline mutations (Yang et al., 2010). Expression studies have shown that some of these mutants have defects like impaired GJ formation, accelerated degradation, or abnormal GJ or $\mathrm{HC}$ function that might contribute to AF pathophysiology (Gollob et al., 2006; Sun et al., 2013; Patel et al., 2014; Gemel et al., 2014b).

6. Cx43 Mutation Linked to Atrial Fibrillation. Cx43 mutations may also be a genetic cause of AF. A single nucleotide deletion (c.932.delC) of the $\mathrm{Cx} 43$ encoding gene (GJA1) was identified by Thibodeau et al. (2010) in DNA prepared from the atrial tissue of a patient with AF (Thibodeau et al., 2010). The mutation resulted in a frame shift, premature truncation, and a $\mathrm{Cx} 43$ polypeptide with impaired cellular trafficking that acted as a dominant negative inhibitor of coexpressed wild-type $\mathrm{Cx} 43$ or $\mathrm{Cx} 40$. Absence of the mutation in patient leukocyte DNA suggested genetic mosaicism.

In most cases, $\mathrm{AF}$ is a secondary event resulting from other cardiac abnormalities like chronic heart failure or valvular disease. In such patients, secondary alterations of $\mathrm{Cx} 40 / \mathrm{Cx} 43$ levels and distributions may contribute to the pathophysiology of AF. About $15 \%$ of AF patients have "lone AF," which develops in apparently normal hearts in the absence of structural abnormalities. It appears that mutations of $\mathrm{Cx} 40$ only rarely cause AF in these patients since they have not been identified very often (Tchou et al., 2012; Gemel et al., 2014a). In contrast, a study of atrial tissue obtained from a group of patients who underwent surgical ablation of their lone AF by Gemel et al. (2014) showed that that reduced $\mathrm{Cx} 40$ levels and heterogeneity of its 
distribution (relative to $\mathrm{Cx} 43$ ) were common in $\mathrm{AF}$ (Gemel et al., 2014a).

\section{Further Genetic Considerations Regarding Gap Junctions and Arrhythmia}

Studies in genetically manipulated mice implicated $\mathrm{Cx} 40$ in various arrhythmias. The initial descriptions of $\mathrm{Cx} 40$-deficient mice showed that they had cardiac conduction abnormalities including atrioventricular and bundle branch block (Simon et al., 1998) or reduced cardiac conduction velocity and predisposition to atrial arrhythmias (Kirchhoff et al., 1998). Subsequent studies confirmed roles for $\mathrm{Cx} 40$ in the specialized conduction system, since $\mathrm{Cx} 40^{-1-}$ mouse hearts exhibit right bundle-branch block, delayed atrioventricular nodal and proximal His bundle conduction, and impaired left bundle conduction (Tamaddon et al., 2000; van Rijen et al., 2001) and in the atrium based on findings of long sinus node recovery time, sinus entry block, slow atrial conduction, and atrial tachyarrhythmias (Kirchhoff et al., 1998; Hagendorff et al., 1999; Verheule et al., 1999). After atrial burst pacing, $\mathrm{Cx} 40^{-/-}$mice develop atrial tachyarrhythmias (Bagwe et al., 2005).

Genetically manipulated mice have also provided convincing evidence for the importance of $\mathrm{Cx} 43$ in ventricular arrhythmias. Gutstein et al. (2001a) generated mice with cardiac-specific loss of $\mathrm{Cx} 43$ (homozygous $\mathrm{Cx} 43$ conditional $\mathrm{KO}$ mice with mosaicism, giving 86\%-95\% decreased expression at 4 weeks age) (Gutstein et al., 2001a). Despite normal heart structure and contractile function, these mice had reduced ventricular conduction velocities and uniformly developed sudden cardiac death from spontaneous ventricular arrhythmias.

However, besides the AF-associated connexin mutations discussed above, there are only a few additional reports linking cardiac connexin mutations to arrhythmias. Makita et al. (2012) identified a Cx40 (GJA5) mutation (encoding the substitution Q58L) in affected members of a family with Progressive Familial Heart Block Type I, a dominant inherited disorder of the HisPurkinje system causing heart block (Makita et al., 2012). Van Norstrand et al. (2012a) identified two missense mutations of $\mathrm{Cx} 43$ (E42K and S272P) among a series of cases of Sudden Infant Death Syndrome (Van Norstrand et al., 2012a).

In contrast, a large number of people carry $\mathrm{Cx} 43$ mutations without any apparent cardiac electrical phenotype. $\mathrm{Cx} 43$ mutations have been identified as the cause of ODDD, an autosomal dominant syndrome that is primarily characterized by craniofacial and limb abnormalities (Paznekas et al., 2003, 2009). Although a large number of different $\mathrm{Cx} 43$ mutations have been identified in ODDD patients (78 to date), cardiac arrhythmias are strikingly absent except for the I130T mutant that has been linked to ventricular tachyarrhythmia (see section VII).
Connexin-related arrhythmias may result from other genetic diseases. Patients with arrhythmogenic cardiomyopathy have an inherited disease characterized by fibro-fatty replacement of the myocardium (especially in the right ventricle) and severe ventricular arrhythmias. The familial form of the disease is linked to mutations of desmosomal proteins (Saffitz, 2011). The arrhythmias may result from loss of GJ plaques as observed in the ventricular tissue of these patients (Kaplan et al., 2004), which, in turn, may result from absent interactions of Cx43 with desmosomal components (Oxford et al., 2007).

We conclude that myocardial GJs and their component connexins (especially $\mathrm{Cx} 40$ and $\mathrm{Cx} 43$ ) are critical for normal cardiac conduction. Their perturbations can contribute to arrhythmias. Rarely, there may be a specific genetic abnormality of the connexin, but, most commonly, the pathogenic changes of the connexins are local and heterogeneous alterations of distribution and abundance that are secondary to other cardiac pathologies.

\section{Cx43 in Oculodentodigital Dysplasia}

Many GJ studies from the eighties were focused on the isolation of the protein that was responsible for junctional intercellular communication in the heart. By using a clever detergent-resistant approach, microgram quantities of pure GJs were obtained from dozens of pooled rat hearts (Manjunath and Page, 1985; Manjunath et al., 1987). Upon examination of the protein content of these purified GJs, a protein of $43 \mathrm{kDa}$ in molecular mass was discovered that appeared as multiple bands that were later defined as phosphorylation species of the cardiac GJ protein (Yancey et al., 1989; Crow et al., 1990; Laird and Revel, 1990; Musil et al., 1990; Laird et al., 1991). In 1987, Beyer et al. (1987) would go on to clone the major cardiac GJ protein and define it as $\mathrm{Cx} 43$. Given the abundance of $\mathrm{Cx} 43$ in the ventricles, $\mathrm{Cx} 43$ was projected to be absolutely critical for the synchronous beating of the ventricular cardiomyocytes and that any attenuation of its expression or function would quickly lead to death. In 1995, when the Kidder and Rossant laboratories reported the first $\mathrm{Cx} 43$ knockout mouse, it was not too surprising that these mice died shortly after birth due to swelling and blockage of the right ventricle outflow tract that caused a failure in pulmonary gas exchange (Reaume et al., 1995). What was probably more surprising was that mice heterozygous for Gja1 gene ablation had no overt cardiac malformation or functional limitations and would go on to live a relatively normal life span (Reaume et al., 1995). Thus, mice could function well on half the normal $\mathrm{Cx} 43$ content in the heart as mentioned earlier (see section VI). These findings began to challenge the paradigm that had emerged over the previous years that an exquisite and tight regulation of $\mathrm{Cx} 43$ in 
the mouse heart was necessary to prevent developmental disorders and cardiac disease. At this time in history, the GJ community was only beginning to become aware of the fact that germline mutations in connexin encoding genes could cause disease. Upon the publication of GJB1 gene mutations linked to X-linked CharcotMarie-Tooth disease (Cochrane et al., 1994), the search was on to see whether humans with GJA1 gene mutations existed in society as many predicted that such mutations would be embryonically lethal and no fetuses would survive through to birth.

\section{A. The Discovery of the Genetic Basis of Oculodentodigital Dysplasia}

In a seminal paper from Ethylin Jabs's laboratory in 2003, they reported the first 17 families that harbored GJA1 gene mutations found in a patient group that was defined as suffering from a rare developmental disorder known as ODDD (Paznekas et al., 2003). Clinically, this disease had been well documented for over several decades, but the genomic cause of this condition had remained elusive (Gorlin et al., 1963; Eidelman et al.,
1967). ODDD is a complex disease as no two patients seem to present with identical disease (Paznekas et al., 2009). Many patients exhibit small eyes, a loss of tooth enamel, fusion of the digits, and craniofacial defects linked to both bone and cartilage (Paznekas et al., 2009). However, at the individual level, patients may be clinically quite diverse and suffer from upwards of a dozen other conditions that include incontinence, skin diseases, thermosensitive skin, glaucoma, and many other syndromes (Paznekas et al., 2009). Importantly, comorbidities that often accompany more classic characteristics of ODDD, like decreased mobility, increase with age and appear to be linked to deteriorations in the central nervous system (De Bock et al., 2013a). Given the ubiquitous distribution of $\mathrm{Cx} 43$ in most human organs, it was equally intriguing to discover what organs were unaffected by GJA1 mutations (Laird, 2014). To that end, the development of the heart and cardiac function is seldom casually affected by a patient harboring a GJA1 gene mutation (Paznekas et al., 2009; Laird, 2014). Over 135 papers have now been published that present the breadth of clinical presentations found

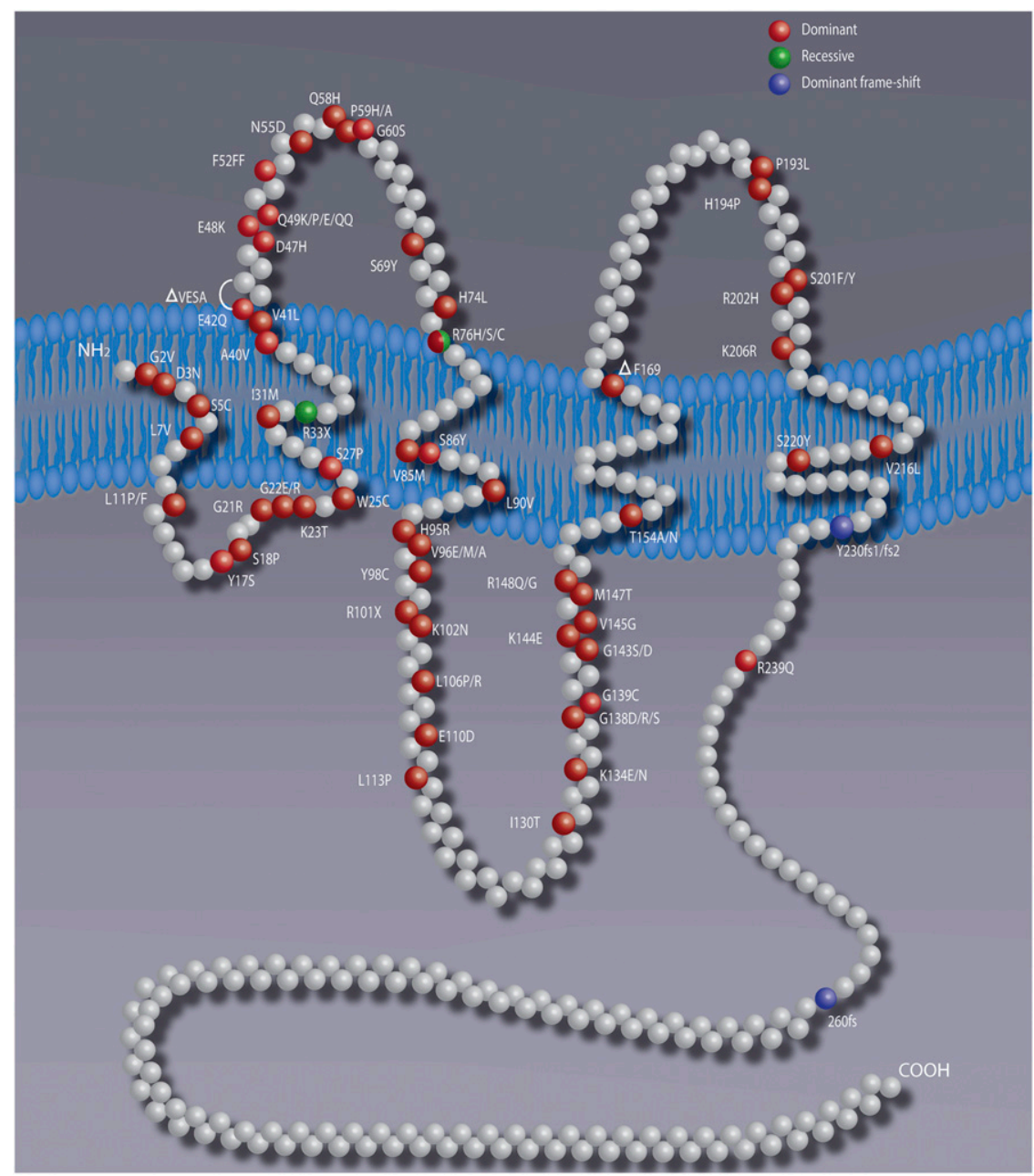

Fig. 8. Composite map of the Cx43 polypeptide depicting all the known GJA1 gene mutations linked to ODDD. 
in patients, the GJA1 mutations that cause ODDD, and the molecular mechanisms that are at the root of the disease.

\section{B. GJA1 Gene Mutations Linked to Oculodentodigital Dysplasia and Beyond}

At last count there are 78 mutations in the GJA1 gene linked to ODDD (Fig. 8) (Laird, 2014). Over 90\% of these mutations are autosomal dominant that mostly result in single amino acid substitutions (Fig. 8). On the first level of interrogation, one might predict that these amino acid substitutions would be substantial changing the charge, hydrophobicity, hydrophilicity, or secondary structure of the motif or domain where they reside. Although that is the case in some instances, it is equally probable that the substituted amino acid is conservative in nature, which is predicted to have minimal effects on the secondary or tertiary structure of $\mathrm{Cx} 43$. The second most intriguing observation when surveying the motifs and domains that harbor $\mathrm{Cx} 43$ mutations is the fact that they are almost entirely located within the first two-thirds of the Cx43 polypeptide sequence (Fig. 8). This observation indirectly suggests that mutations in the GJA1 gene that encode the C-terminal domain of $\mathrm{Cx} 43$ are probably embryonically lethal. This notion is not entirely true, because three autosomal dominant frame-shift mutations have been identified at residues 230 (two different mutations) and 260 , suggesting that human survival is indeed possible if the $\mathrm{C}$ terminus is ablated from one allele, although very few patients worldwide have been identified with this class of mutations (Laird, 2014). Two autosomal recessive mutations have also been identified and linked to ODDD, one mutation truncates $\mathrm{Cx} 43$ at residue 33 and a second mutation causes an amino acid substitution at residue 76 (Paznekas et al., 2009; Laird, 2014). In the case of the homozygous mutation that leads to a severe truncation of $\mathrm{Cx} 43$ at residue 33 , which is the equivalent to a $\mathrm{Cx} 43$ knockout in humans, these young children were severely ill and exhibited multisystem functional disorders (Paznekas et al., 2009).

Although $100 \%$ of ODDD patients genotyped harbor GJA1 gene mutations, there are a few cases of GJA1 gene mutations that do not cause ODDD. For example, the autosomal recessively inherited R239Q mutant has been shown to cause a related syndrome to ODDD called craniometaphyseal dysplasia ( $\mathrm{Hu}$ et al., 2013). Two other mutants (E42K and S272P) have been linked to sudden infant death by mechanisms that remain unclear, inasmuch as the E42K mutant has perturbed $\mathrm{Cx} 43$ properties while the $\mathrm{S} 272 \mathrm{P}$ appears to function normally (Van Norstrand et al., 2012b; Lübkemeier et al., 2015). Still other mutations as found in patients homozygous for the $\mathrm{R} 76 \mathrm{H}$ amino acid substitution exhibit ODDD but have also been classified to present with Hallermann-Streiff syndrome characterized by a small stature, congenital cataracts, hypotrichosis, beaked nose, skeletal anomalies, and teeth defects (Pizzuti et al., 2004). Thus, some caution must be exercised in concluding that all mutations in the GJA1 gene will result in patients presenting with ODDD or ODDD only.

\section{Molecular Mechanisms Linking GJA1 Gene Mutations to Oculodentodigital Dysplasia}

Over two dozen ODDD-linked $\mathrm{Cx} 43$ mutants have been engineered and interrogated when expressed in reference cells or tissue-relevant cellular environments (De Bock et al., 2013a; Laird, 2014). In some cases, these host cells coexpressed, or were engineered to express, $\mathrm{Cx} 43$ in an attempt to mimic the autosomal dominant mode of disease inheritance, or the host cells expressed other members of the large connexin family of 21 genes (De Bock et al., 2013a; Laird, 2014). In all ectopic mutant-expressing situations, it is challenging to create physiological relevant ratios of $\mathrm{Cx} 43$ to disease-linked Cx43 mutants and also to have appropriate levels of Cx43 mutants coexpressed with native levels of other connexin family members. Nevertheless, these strategies were successfully used to determine that the mechanisms responsible for $\mathrm{Cx} 43$ mutants causing disease are highly variable and can be first classified into either loss-of-function or gain-of-function mutants (Shibayama et al., 2005; Laird, 2006, 2008, 2014; De Bock et al., 2013a). Loss-of-function mutations are found throughout much of the polypeptide sequence and include many amino acid substitutions within the amino terminal of $\mathrm{Cx} 43$ where the resulting mutants could traffic to the cell surface and assemble into GJ-like structures, but these channels were not functional (e.g., G21R) (Roscoe et al., 2005; Gong et al., 2007; Shao et al., 2012). Other loss-of-function mutants included frame-shift mutations that caused the $\mathrm{C}$ terminus of $\mathrm{Cx} 43$ to be lost, and these mutants were retained within the endoplasmic reticulum or Golgi apparatus because they failed the quality control assessment to be able to traffic to the cell surface (e.g., fs230, fs260) (Gong et al., 2006; Churko et al., 2010). Still other loss-offunction mutants assembled into channels, but the resulting channels, both GJ and $\mathrm{HCs}$, were not fully functional, suggesting changes in channel pore or gating mechanisms (e.g., Y17S, G21R, A40V, L90V, and I130T illustrated in Fig. 1) (Lai et al., 2006; Kalcheva et al., 2007; Churko et al., 2010; Lorentz et al., 2012; De Bock et al., 2013a; Stewart et al., 2013; Huang et al., 2014). Other loss-of-function ODDDlinked mutants cannot be assigned to one category and exhibited combinatory defects in trafficking, assembly, and/or function.

Somewhat less expected, ODDD-linked mutants acquired new or enhanced properties that can be best described as gain-of-function mutants. For example, several ODDD-linked mutants (I31M, G60S, G138R, and G143S illustrated in Fig. 1) exhibited a tendency to 
acquire an open $\mathrm{HC}$ state or form leaky HCs, suggesting that the tight regulation of maintaining HCs closed at the cell surface was dysfunctional; interestingly this gain-of-function of $\mathrm{HCs}$ was associated with decreased function of GJs (Dobrowolski et al., 2007; Dobrowolski et al., 2008; Kozoriz et al., 2013). Still other ODDD mutants may acquire the ability to interact with other coexpressed connexin family members. Concrete examples of this are difficult to pinpoint in ODDD but are more commonly found in Cx26-linked skin diseases where Cx26 mutants can surprisingly acquire the ability to impair the function of $\mathrm{Cx} 43$, a connexin not typically able to bind Cx26 (Rouan et al., 2001; Thomas et al., 2004). Since Cx43 is thought to interact with $\mathrm{Cx} 40$ and $\mathrm{Cx} 45$ (Laing et al., 2001; Bouvier et al., 2009), when considering the heart, one might propose that $\mathrm{Cx} 43$ mutants may lose the ability to bind $\mathrm{Cx} 40$ or $\mathrm{Cx} 45$, thus allowing these cardiac connexins to rescue the heart from disease in ODDD patients. Other gain-of-function mechanisms where mutants acquire the ability to bind novel proteins have been proposed, but definitive examples of this mechanisms are not well established.

\section{Insights from Mouse Models}

Given that ODDD is typically an autosomal dominant disease, the need emerged to generate genetically modified mice that were heterozygous for the $\mathrm{Cx} 43$ gene mutation and thus mimic the human condition. Through the use of an $N$-ethyl- $N$-nitrosurea mutagenesis screen, in 2005 Janet Rossant and team identified a Cx43 G60S mutant mouse that mimicked human ODDD (Flenniken et al., 2005). Although this particular mutant has never been found in the human population, the mutant mouse mirrored the human disease as revealed by fusion of the digits, loss of tooth enamel, small eyes, and craniofacial bone defects (Flenniken et al., 2005). This mouse has been used as a surrogate of human ODDD in nearly two dozen publications that have served to uncover how a loss-of-function $\mathrm{Cx} 43$ mutant causes disease or defects in bone, teeth, reproductive organs, bladder, eyes, and the CNS while sparing other Cx43-rich tissues like the heart and vessels (Toth et al., 2010; Tsui et al., 2011; Lorentz et al., 2012; Kozoriz et al., 2013; Stewart et al., 2013; Winterhager et al., 2013; Zappitelli et al., 2015).

In addition to ODDD patients presenting with multisystem syndromes, it was noted that a few $\mathrm{Cx} 43$ gene mutations appeared possibly to predispose patients to potential cardiac defects (Paznekas et al., 2003, 2009). One of these mutations was I130T, which was engineered into a mouse model of ODDD to investigate if this particular $\mathrm{Cx} 43$ mutant would lead to notable cardiac dysfunctions including arrhythmias (Kalcheva et al., 2007). Here the authors found that these mice did have significantly less $\mathrm{Cx} 43$ in the functional myocardium that corresponded with less functional coupling and slowing of conduction velocity (Kalcheva et al.,
2007). Furthermore, these mice did exhibit increased susceptibility to ventricular tachyarrhythmias (Kalcheva et al., 2007). Unfortunately, there is not a sufficient number of patients that harbor the I130T mutation to determine if these clinical presentations are consistently present in humans that harbor this mutation. However, these studies open up the possibility that one or more ODDD-linked mutants may in fact cause a cardiac phenotype.

A third mouse that mimics ODDD was generated by conditionally knocking in the $\mathrm{Cx} 43$ G138R mutant, which allows for both unaffected and affected organs to be examined in a tissue-specific manner (Dobrowolski et al., 2008). By using this strategy, mice that selectively expressed the mutant in cardiomyocytes developed spontaneous arrhythmias that were found to correlate with a reduction in $\mathrm{Cx} 43$ GJ function (Dobrowolski et al., 2008). In this case, the authors concluded that changes in rhythmogenesis were due to increases in ATP release through $\mathrm{Cx} 43 \mathrm{HCs}$ that had become leaky due to the mutation. Here, the proposed gain-offunction induced by the G138R mutant appeared to be responsible for the ODDD phenotype (Dobrowolski et al., 2007). In another study, mice conditionally expressing the G138R mutant in the limb bud developed syndactylies due to impaired apoptosis, a condition linked to reduced expression of sonic hedgehog and bone morphogenic protein 2 (Dobrowolski et al., 2009). These later studies point to a possible second mechanism of how the G138R mutant can lead to tissue changes that present in ODDD-linked phenotypes. In still another study, when the G138R mutant was conditionally expressed in cells of the chondroosteogenic lineage using the Dermo1 promoter, mutant mice were found to suffer from osteopenia (Watkins et al., 2011). Mechanistically, osteopenia was found to be due to high bone turnover, increased osteoclastmedicate bone resorption as a consequence of increased osteoclastogenesis, and defective osteoblast differentiation (Watkins et al., 2011). Thus, $\mathrm{Cx} 43$ mutants may cause impairments in multiple pathways involved in organogenesis. Collectively, mutant mouse models of ODDD have led to key discoveries that not only link Cx43 to diseases but address the need for a full complement of $\mathrm{Cx} 43$ function in several key developmental processes.

\section{E. Next-Generation Human Models of Oculodentodigital Dysplasia}

Further advancements in our understanding of the genetic and mechanistic basis of ODDD have now benefited from new models that may better represent this multiorgan-affected syndrome. To this end, ODDD patients worldwide have generously donated skin biopsies for the purpose of establishing patient-based dermal fibroblasts. Given that these patient-derived dermal fibroblast have one normal and one mutant 
allele that each express $\mathrm{Cx} 43$ from its native promoter, it is expected that both gene transcription and translation would be as found in the patient. Importantly, it is also possible to obtain dermal fibroblasts from unaffected relatives of the patients in an attempt to control for genetic diversity found within the human population. By using this approach it was discovered that patients encoding the D3N and V216L Cx43 mutants have distinct changes in $\mathrm{Cx} 43$ phosphorylation and expression levels and these changes may lead to subclinical defects in wound repair (Churko et al., 2011). Other studies reported that dermal fibroblasts harboring D3N or V216L Cx43 mutants caused the reprogramming and expression of extracellular matrix genes that may serve key roles in skin wound repair (Esseltine et al., 2015). Further studies using dermal fibroblasts from patients harboring L7V, G138R, and G143S Cx43 mutants revealed that every mutation caused distinct molecular changes in $\mathrm{Cx} 43$ localization, assembly, or function (Kelly et al., 2016). In the case of G143S, it retained a functional state not unlike wildtype $\mathrm{Cx} 43$, raising questions as to the molecular mechanism responsible for this mutation to cause ODDD (Kelly et al., 2016). In summation, consistent with studies from reference cells and mutant mice, studies from patient-derived cells revealed that there appears to be several classes of $\mathrm{Cx} 43$ gene mutations that cause ODDD by distinct mechanisms leading to mutation-specific pathologies. Importantly, depending on how a specific mutation causes disease, they may be amenable for therapeutic interventions as we enter the dawn of new gene editing technologies.

Although ODDD remains a rare disease, understanding the etiology of this disease is critically important, because it involves mutations in the most commonly found connexin gene in humans, GJA1. Thus, understanding how mutations in this $\mathrm{Cx} 43$ encoding gene cause disease also provides critical insights into the role of $\mathrm{Cx} 43$ in any of the over 50 distinct cell types where it is expressed. Surprisingly, some of these $\mathrm{Cx} 43$-rich cells and tissues remain unaffected by loss- or gain-of-Cx43 function, further elucidating the redundancies and compensatory mechanisms that protect the human body from developmental abnormalities and disease. Looking forward, one wonders if we can effectively treat this genetic disease in patients where the disease appears to increase in severity during aging for reasons that remain poorly understood. Cx43-regulating therapeutic drugs remain sparse as they have begun to enter stage 2 and 3 clinical trials but have yet to become a mainstay in any disease or injury treatment (Kelly et al., 2016). Perhaps the future rests in gene editing, where CRISPR-Cas9 could be used to repair ODDDlinked GJA1 gene mutations during development or in affected organs. To this end, we have now reprogrammed ODDD patient-derived fibroblasts into inducible pluripotent stem cells and are assessing the role of a full complement of functional $\mathrm{Cx} 43$ in osteogenesis, a process often affected in ODDD patients (Esseltine et al., 2017). Complementary approaches are also being developed to repair the GJA1 gene in these patient-derived stem cells as a proof-ofprincipal that ODDD is a fixable syndrome. While it is still early days for therapeutic applications of gene repair, there is new hope that patients with defined GJA1 mutations can be effectively treated in the decades to come.

\section{Connexins in Cerebrovascular and Retinovascular Disease}

The central nervous system (CNS) comprises multiple cell types arrayed in complex interconnections which require sustained and efficient vascular supply of oxygen, glucose and numerous nutrients and metabolites. One way these various cell types interact is through GJs and HCs in specific ways related to their functional roles (Decrock et al., 2015). Within the mammalian brain, the various cell types express over ten different connexins, providing a network of diverse and complex intercellular communication. These channels have distinct functions within the different cell types, and their expression can change dramatically during neurodevelopment (Cina et al., 2007) and injury (Freitas-Andrade and Naus, 2016).

The vascular cells express connexins (Cx37, Cx40, Cx43) (De Bock et al., 2011, 2013b) that contribute to interendothelial coupling. These play an important role in blood-brain barrier function, as demonstrated using $\mathrm{Cx} 30^{-/-}$mice crossed with $\mathrm{Cx} 43^{\mathrm{fl} / \mathrm{fl}} / \mathrm{hGFAP}-\mathrm{Cre}$ (global $\mathrm{Cx} 30 \mathrm{KO}$ and astrocyte-specific $\mathrm{Cx} 43 \mathrm{KO}$ ) (Ezan et al., 2012). These mice show compromise of the blood-brain barrier under stress due to increased hydrostatic vascular pressure and shear stress, swelling of astrocytic endfeet due to edema, as well as reduced expression of aquaporin-4, forming water channels, and $\beta$-dystroglycan, involved in anchoring astrocyte endfeet to the perivascular basal lamina. Endothelial functions are closely regulated by junctional interactions with astrocytes; specifically important are the connexins expressed in astrocytic endfeet (Froger et al., 2010; Pannasch et al., 2011). Astrocytes are physically separated from vascular endothelial cells by a continuous basal lamina; hence, the connexins present at this endothelial-astrocyte interface do not form GJs, at least not under normal conditions. Although $\mathrm{Cx} 43$ expression is very low in blood-brain barrier endothelial cells under normal conditions, astrocytic endfeet display highly prominent $\mathrm{Cx} 43$ expression (Simard et al., 2003). Endfeet-located $\mathrm{Cx} 43$ forms GJs that couple adjacent endfeet that facilitate $\mathrm{Ca}^{2+}$ wave propagation along the astrocyte lining of small blood vessels (Mulligan and MacVicar, 2004). Although there is little evidence for endothelial-astrocytic GJs, pericytes also express Cx43, 
which has been proposed to form GJs with astrocytes as well as endothelial cells (Wakui et al., 2006; Winkler et al., 2011). Specifically, pericytes contact endothelial cells via so called peg-and-socket junctions that are extensions from one cell invaginating another cell and that been reported to be immunopositive for connexins (Vigmond et al., 2000; Caruso et al., 2009; see also De Bock et al., 2014b).

The main parenchymal connexins, $\mathrm{Cx} 43$ and $\mathrm{Cx} 30$ (Nagy et al., 1999), are highly expressed in astrocytes, while Cx26 has also been reported (Nagy et al., 2001). GJs form a functional syncytium of coupled astrocytes, contributing to spatial buffering, in dealing with elevated concentrations of extracellular potassium ions $\left(\mathrm{K}^{+}\right)$during increased neuronal activity; GJs assist in dispersal of $\mathrm{K}^{+}$accumulated by astrocytes (Walz and Hertz, 1983). The role of astrocytic $\mathrm{Cx} 43$ has been extensively examined in the context of GJs functioning to spatially buffer potassium and glutamate (Wallraff et al., 2006; Giaume et al., 2010), whereas Cx30 modulates astrocyte glutamate transport, thereby controlling hippocampal excitatory synaptic transmission (Pannasch et al., 2014). It was also shown that Cx30 controls the morphology of astrocytic processes at the synaptic cleft, and this was associated with altered glutamate clearance by astrocytes (Pannasch et al., 2014).

GJs in oligodendrocytes (Cx32, Cx29, Cx47) have been shown to be essential for proper myelination (Menichella et al., 2003), as well as potassium buffering (Menichella et al., 2006). Although microglia have been reported to express $\mathrm{Cx} 43$ and form GJs (Eugenín et al., 2001), others have not observed $\mathrm{Cx} 43$ immunoreactivity in microglia (Mei et al., 2010; Theodoric et al., 2012). Others have demonstrated that microglial Cx43 does not form GJs (Wasseff and Scherer, 2015) but rather HCs (Takeuchi et al., 2006). Expression of other connexins in microglia has also been described, including Cx32 (Dobrenis et al., 2005; Maezawa and Jin, 2010).

Depending upon the maturity of the CNS, neurons can express seven different connexins. These include Cx36, which is most highly expressed in neurons (Condorelli et al., 1998; Belluardo et al., 2000; Venance et al., 2000; Rash et al., 2012), as well as Cx30.2 (Kreuzberg et al., 2008), Cx31.1 (Dere et al., 2008), Cx40 (Personius et al., 2007), Cx45 ( $\mathrm{Li}$ et al., 2008a), Cx50, and $\mathrm{Cx} 57$ in the retina (Puller et al., 2009). This presumably reflects the diversity of neuronal cell types, expressing a range of connexins with varying functions in the developing CNS. However, specific knockout of the prototypic neuronal Cx36 in mice results in near complete loss of neuronal GJC in the mature CNS, indicating that it is the primary neuronal connexin (Deans et al., 2001; Hormuzdi et al., 2001; Long et al., 2002; De Zeeuw et al., 2003).

\section{A. Inflammation and Reduced Vascular Integrity in Cerebrovascular and Retinovascular Disease}

When considering connexins in cerebrovascular and retinovascular disease, two pathogenic aspects appear to be important. The first aspect concerns inflammation and its effect on vascular integrity. The second is the switch from an initial inflammatory response to a self-perpetuating innate immune system, commonly involving the inflammasome pathway and resulting in a chronic inflammatory condition that is typical for chronic cerebrovascular and retinovascular disease. All of these have a connexin HC component, with interventions in a number of animal models improving outcomes, but with the role of direct cell-cell coupling via GJs being more complex. There may, for example, be multiple roles for connexins at the endothelial-glial interface, taking into account GJ coupling, HCs, and roles of connexins in pericytes and in endothelial mitochondria (Trudeau et al., 2012).

Two recent reviews detailed the conflicting evidence for the effect of inflammatory conditions on connexin transcription/protein translation and GJ function (Kim et al., 2016b; Willebrords et al., 2016). Analysis of publications cited in the Willebrords review shows that Cx43 levels (mRNA or protein) were reduced in 15 studies involving infection or the addition of inflammatory cytokines, but increased in 10 studies. Within a study, different cell types may respond differently. This variability in results reflects the diversity of models, cell types, time course, and dosing used and in vitro and in vivo responses may vary and even cells from different regions of a tissue may respond differently (Bennett et al., 2012). In addition, although "bystander death" is considered to be a GJ phenomenon [the term was originally coined to describe tumor cell-induced apoptosis in neighboring cells during coculture (Freeman et al., 1993)], Cx43 gene knockout and consequent loss of GJ coupling exacerbate injury size in the CNS (Siushansian et al., 2001; Nakase et al., 2003), indicating that maintenance of a functional astrocytic syncytium is essential or $\mathrm{Cx} 43$ has other non-channelrelated functions.

On the other hand, connexin HCs, which are tightly controlled under normal conditions with low opening probability, appear to have a more consistent role in CNS inflammatory responses, forming a "pathologic pore" under injury or inflammatory conditions (Decrock et al., 2009b, 2015; Vinken, 2015). HC opening may provoke 1) ion fluxes that challenge osmoregulation; 2) the release of ATP, glutamate, and lactate that may contribute to neuronal loss; 3) breach of the blood-brain barrier or blood-retina barrier; and 4) initiation and perpetuation of inflammatory cycles. Once released, ATP triggers $\mathrm{Ca}^{2+}$ waves and further ATP release (Bennett et al., 2003; Neary et al., 2003; Suadicani et al., 2004; Scemes and Giaume, 2006), mediating 
secondary damage distal to the trauma zone (Rovegno et al., 2015). In terms of inflammation, HC opening can be initiated by bacterial LPS or peptidoglycan (De Vuyst et al., 2007; Robertson et al., 2010), with HC opening considered to be an initiating step in the innate immune system. Release of ATP increases Toll-like receptor 2 and Il-6 production (Robertson et al., 2010) and UDP release may act as a danger signal from stressed or apoptotic cells (Qin et al., 2016). Inflammatory conditions typically promote $\mathrm{HC}$ opening while reducing GJ communication (see section II.E.5.b). Decreased cell-cell coupling may serve to deliberately isolate viral or bacterial infected cells. Increased vascular wall permeability correlates with a rapid immune response [such as neutrophil invasion (Cronin et al., 2008)], but vessel die back also isolates vessels from a possible source of infection, reducing the risk of septicemia. Inflammation is a double-edged sword though, and a hyperactive response exacerbates damage to both inflamed and surrounding tissue (Lehnardt et al., 2003). In the majority of wounds with modern hygiene, in most spinal cord and brain trauma (where the tissue is not exposed), and in virtually all CNS chronic disease conditions, this immune response becomes excessive or worse, perpetuates the inflammatory response, despite the absence of infection. Although pretreatment with the GJ blocker exacerbated infection in a model of peritonitis (Qin et al., 2016), no animal studies involving HC block have to date shown increased signs of infection.

\section{B. Chronic Inflammatory Disease}

Independently of GJ and $\mathrm{HC}$ roles in acute CNS injury, it is becoming apparent that perpetuation of chronic inflammatory conditions may be connexin HC mediated. Many chronic inflammatory diseases are associated with vascular dropout or degeneration. Vascular degeneration is a feature of inflammatory neurodegenerative diseases such as Alzheimer's and Parkinson's, with Alzheimer's the most common neurodegenerative condition seen in aging populations and Parkinson's second. In the eye, age-related macular degeneration affects as many as one in seven over the age of 50 in developed nations and diabetic retinopathy affects over $15 \%$ of Caucasians. Its prevalence is, however, three times higher in some populations such as New Zealand Māori and Pacific peoples. Macular edema and open angle glaucoma are likewise serious eye conditions associated with vascular degeneration. Although neovascularization is typical of the wet form of macular degeneration (about 10\%-15\% of all agerelated macular degeneration) and diabetic retinopathy, inflammation and vessel dropout is increasingly considered to be the underlying cause of the disease and subsequent vision loss in those patients.

Chronic inflammation in these conditions would appear to involve a switch to the innate immune system's inflammasome pathways. ATP and its metabolic derivatives ADP, AMP, and adenosine are key inflammasome activators through P2XR, P1YR, and P1R receptor binding to trigger the NLRP3 inflammasome pathway (Riteau et al., 2012; Baron et al., 2015). Damage-associated molecular patterns (such as proinflammatory cytokines, chemokines, reactive oxygen species, and ATP), pathogen-associated molecular patterns (such as lipopolysaccharides, pathogens, irritants, and peptidiglycans), or TNF- $\alpha$ may activate the inflammasome pathway via the nuclear factor $-\kappa B$ complex. The end result is activation of caspase- 1 and cleavage of the proinflammatory cytokines IL- $1 \beta$ and IL-18 into their mature forms and release from the cell. Increased TNF- $\alpha$ and IL- 6 release also occurs, along with a new round of ATP release (for review, see Kim et al., 2016b; Willebrords et al., 2016; Zhou et al., 2016). There is a wealth of evidence for connexin HC roles in each of the inflammasome pathway stages: initiation, inflammatory cytokine release, and perpetuation. Connexin $\mathrm{HC}$ opening can facilitate the release of several molecules, including ATP, glutamate, aspartate, and lactate; the bidirectional flow of ions; and can lead to the onset of astroglial intercellular $\mathrm{Ca}^{2+}$ waves that contribute to neurodegeneration (Wang et al., 2013a; Castellano and Eugenin, 2014; Lohman and Isakson, 2014; De Bock et al., 2014a; Karagiannis et al., 2016). Furthermore, connexin HC opening probability is increased in response to injury and inflammation, and this has been correlated with the release of inflammatory cytokines such as TNF- $\alpha$, IL-18, and IL- $1 \beta$ and the induction of IL- 6 and TLR2 mRNA expression. If the innate immune response is unsuccessful in clearing a disease stimulus, short-term elevation of proinflammatory mediators can progress to chronic, self-perpetuating inflammation characteristic of the diseases mentioned above. It can be difficult to distinguish between GJ intercellular communication and HC roles in inflammatory disease, but analysis of studies cited in one recent review (Willebrords et al., 2016) showed that with disease, injury, or inflammatory cytokine addition, GJ intercellular coupling was decreased in 18 studies, with only 3 showing a coupling increase. Conversely, where $\mathrm{HC}$ activity was measured, all 12 studies revealed increased activity.

Both connexin HCs and the related pannexin channels may contribute to pathologic ATP release with the contribution that each makes often difficult to dissect (Bennett et al., 2012; Montero and Orellana, 2015). In one study though, pannexin channels were shown to be associated with ATP release during apoptosis but not for inflammasome activation ( $Q u$ et al., 2011). In an in vitro endothelial cell hypoxia model, two-thirds of the ATP release was through connexin $\mathrm{HCs}$ and onethird through pannexin channels, a combination of $\mathrm{HC}$ block [using Peptide 5 (O'Carroll et al., 2008)] and probenecid blocking all hypoxia-induced ATP release (and matching the effect of the nonspecific channel 
blocker carbenoxolone) (Kim and Green, 2016). With reperfusion (return to normal medium), ATP continued to be released through connexin $\mathrm{HCs}$ but there was now no contribution from pannexin channels. In an Alzheimer's disease animal model, inflammation triggered pannexin channel activity but only within a subpopulation of reactive astrocytes in direct contact with amyloid plaques. $\mathrm{Cx} 43 \mathrm{HCs}$ were a wider contributor to ATP (and glutamate) release (Yi et al., 2016). In a series of studies (Cea et al., 2013, 2016a,c), inflammasome activation was demonstrated to be $\mathrm{Cx} 43$ and Cx45 HC mediated, leading to skeletal muscle atrophy in a Duchene muscular dystrophy model or after sustained high dose glucocorticoid treatments.

The immune system has a variety of defenses against pathogen-associated molecular patterns and damageassociated molecular patterns, and connexin expression and function has been documented in the immune and lymphatic system (Oviedo-Orta and Howard Evans, 2004; Neijssen et al., 2007). Cx43 appears to play a role in neutrophil recruitment and $\mathrm{T}$ cell-activation (Glass et al., 2015) and is involved in B cell activation, spreading, and migration (Machtaler et al., 2011). Knockdown of $\mathrm{Cx} 43$ in animal models of skin wound and spinal cord injury, for example, significantly reduces neutrophil counts and subsequent macrophage invasion into wound sites (Qiu et al., 2003; Cronin et al., 2008). Once the inflammasome is activated, maturation and release of proinflammatory cytokines (although too large to pass through the $\mathrm{HC}$ itself) are modulated by connexin $\mathrm{HCs}$, and conversely, inflammatory cytokines increase $\mathrm{HC}$ activity. In a spinal cord contusion model, Peptide5 delivered by osmotic mini-pump to the injury site significantly reduced both TNF- $\alpha$ and IL- $1 \beta$ levels compared with untreated controls when assessed 8 hours after the injury (O'Carroll et al., 2013). There was less lesion spread in treated animal lesions, but IL- $1 \beta$ was at sham control levels. It is difficult to show if this is a direct HC modulatory effect or whether the lowered cytokine levels may result from reduced ATP release in the first place, but activated microglia are known to secrete TNF- $\alpha$ and IL- $1 \beta$, which triggers HC opening in astrocytes (Bennett et al., 2012). The astrocytes then release ATP, causing further inflammasome activation, and increase extracellular glutamate by decreased uptake via $\mathrm{Na}^{+}$-dependent transporters or directly release glutamate, which can kill neurons by excitotoxic signaling. Astrocytes are therefore both instigator and effector and key players in infectious and neurodegenerative diseases of the CNS (Kielian, 2008; Quintanilla et al., 2012). Figure 9 gives an overview of $\mathrm{HC}$ roles in injury spread and the perpetuation of chronic disease.

\section{Central Nervous System Injury Models}

1. Brain and Spinal Cord. Preterm ischemia is a cause of cerebral palsy. Studies in a large animal (sheep) model indicate a strong $\mathrm{HC}$ role in subsequent events. Although there is no evidence for a loss of vascular integrity, the model, with 24 hours-a-day, 7-day continuous recording of multiple parameters has provided insights into $\mathrm{HC}$ roles and treatment regimens for acute CNS injury. The model involves exteriorization of the lamb and the insertion of catheters or electrodes to enable measurement of mean arterial blood pressure and blood flow rate, heart rate, electroencephalographic activity, brain swelling, and body temperature. Inflatable carotid occluder cuffs are placed around the carotid arteries and a catheter is placed into the left lateral ventricle of the brain to allow treatment delivery (for further details, see Davidson et al., 2012b). In a variation of the model, preterm asphyxia was modeled in a similar manner but with an inflatable cuff placed around the umbilical cord instead of the carotid arteries. The carotid artery cuffs were inflated for 30 minutes to create severe ischemia, and then in the asphyxia model the umbilical cord cuff was inflated for 25 minutes.

Peptide5 delivery at high doses $(1250 \mu \mathrm{mol} / \mathrm{kg}$ over 25 hours) was reported to have adverse effects, most likely attributable to GJ uncoupling. In those animals, greater brain swelling was evident, with a trend toward higher lactate concentrations and mortality (Davidson et al., 2012a). However, HC-blocking doses (50 $\mu \mathrm{mol} / \mathrm{kg}$ for the first hour and $50 \mu \mathrm{mol} / \mathrm{kg}$ spread over the subsequent 24 hours) significantly improved outcomes (Davidson et al., 2012b). Electroencephalographic activity improved, seizures were reduced with less status epilepticus, there was earlier return to normal sleep cycling, increased survival of oligodendrocytes in intragyral and periventricular brain matter, and increased brain weight. All of those parameters were statistically significant with an intermediate increase in surviving neurons. After asphyxia in preterm fetuses, HC block with the same regimen gave earlier recovery of electroencephalographic power, was neuroprotective, and improved recovery of oligodendrocyte maturation (Davidson et al., 2014). With preterm ischemia, seizures and brain swelling starts about 6 hours, correlating with increased $\mathrm{Cx} 43$ expression, and in both models the peptide delivery was started 90 minutes after the insult to provide a therapeutic window of opportunity. When the start of treatment is delayed until 3 hours after the insult, HC blockade reduced seizure burden but no longer had effect on electroencephalographic power or histology (Davidson et al., 2015). These studies suggest that for these parameters at least, HC block but not GJ uncoupling may be crucial, and there is a window of opportunity for treatment that may be as short as 3 hours for acute injury [although this does not apply in chronic disease indications where connexin channel modulation treatment, in one report 8 weeks after injury (Ormonde et al., 2012), can be used to break the inflammatory cycle]. 


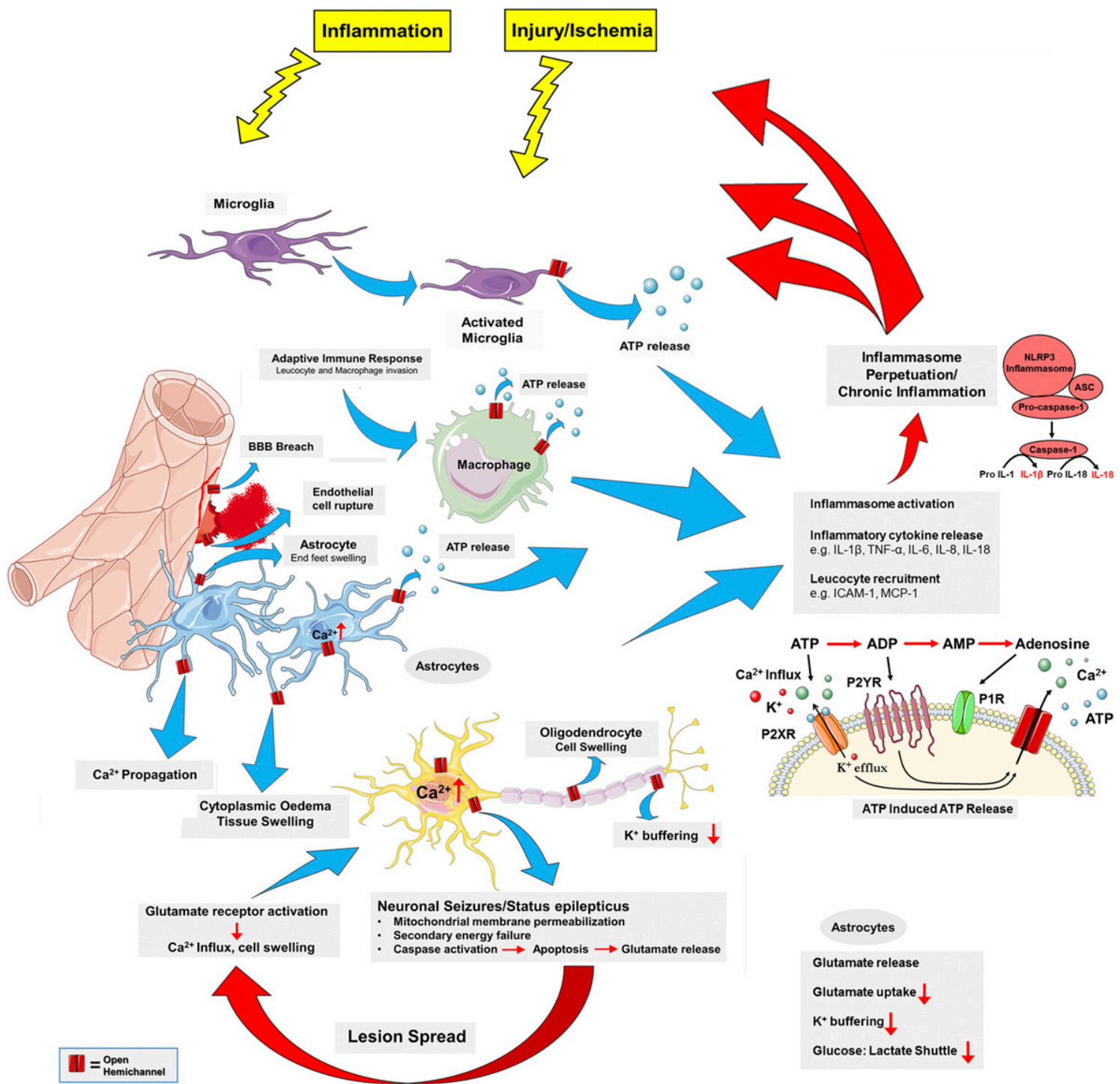

Fig. 9. Hemichannel-linked "pathologic pore" roles in injury spread and the perpetuation of chronic disease. Hemichannel opening plays a key role in cellular edema, lesion spread, initiation, and perpetuation of both the adaptive and innate immune responses and in particular in chronic disease conditions and the initiation and perpetuation of the inflammasome pathway. Multiple pathologic signals have been shown to trigger HC opening, resulting in pathologic levels of extracellular inflammatory stimulators such as ATP (with subsequent ATP-induced ATP release) and glutamate, the onset of calcium waves and seizures, and changes in the cytoplasmic ionic composition that may trigger apoptosis or loss of a cell's ability to osmoregulate. Loss of vascular integrity to breach the blood-brain or blood-retina barrier may be an initiating event, but conversely, inflammation leads to loss of vascular integrity, including rupture of endothelial cells and vascular dropout. The blue arrows to adjacent boxes indicate reported outcomes of $\mathrm{HC}$ opening in different cell types (irrespective of the specific connexin isoform expressed). Not all effects necessarily occur at once, depending upon the type and extent of injury and the specific cell type involved. These pathways have been implicated in a remarkable number of central nervous system cerebrovascular and retinovascular indications, including trauma, such as spinal cord injury; ischemia; stroke; and infectious disease and chronic diseases, including Alzheimer's, Parkinson's, amyotrophic lateral sclerosis, diabetic retinopathy, macular edema, age-related macular degeneration, and chronic pain. In several models, intervention using connexin HC blockers has been exploited to break the cycle, reducing the extent of damage after an acute insult or breaking the inflammatory cycle in chronic disease conditions. Red arrows at the (top) and (bottom) emphasize the HC-mediated feedback loops that contribute to damage spread and perpetuation of the inflammatory response.

Spinal cord injury intervention studies based on $\mathrm{Cx} 43$ knockdown with oligonucleotides have also revealed roles of connexins, as a protein or a channel, in CNS lesion spread, edema and inflammation, and in loss of vascular integrity that further exacerbate the damage. Spinal cord segments placed into organotypic culture swell despite the absence of a patent blood supply, and tissue dies back from the cut edges. Treated with $\mathrm{Cx} 43-$ specific antisense oligodeoxynucleotides to block $\mathrm{Cx} 43$ protein translation, a reduction in segment swelling and improvement in neuron survival was observed, with ex vivo cord survival for up to 5 days (Zhang et al., 2010). This is also apparent with connexin mimetic peptide, with the ex vivo model in conjunction with in vitro cell culture models (dye uptake and dye spread) demonstrating that these effects are $\mathrm{HC}$ mediated (O'Carroll et al., 2008). In vivo, there are three rat spinal cord injury studies demonstrating that connexin channel modulation significantly improves outcomes. In the first, using $\mathrm{Cx} 43$-specific antisense 
oligodeoxynucleotides after a compression injury, behavioral tests showed improved locomotion within 24 hours, with continued improvement for 4 weeks (when the study was terminated). Treated cords had significantly less edema and lesion spread, less inflammation (both neutrophil invasion and astrocytosis), and a dramatic reduction in vascular hemorrhage, which otherwise occurs for more than $4 \mathrm{~mm}$ either side of the injury site (Cronin et al., 2008). After partial spinal cord transection too, the $\mathrm{Cx} 43$ antisense treatment reduced inflammation, including microglial activation in and around the lesion site (Cronin et al., 2008). In a separate study, $\mathrm{Cx} 43$ mimetic peptides (Peptide5) also improved locomotor outcomes up to 5 weeks postcontusion injury. The treatments (delivered via an intrathecal catheter and osmotic minipump starting 1 hour postinjury) decreased levels of TNF- $\alpha$ and IL- $1 \beta$ in the wound bed 8 hours postinjury, with a subsequent reduction in astrocytosis and activated microglia as well as an increase in motoneuron survival (O'Carroll et al., 2013). Clinically this method of peptide delivery is less likely to be acceptable, and a third study, with a contusion injury, instead used systemic delivery (three intraperitoneal injections at 2-hour spacings) of the Cx43 mimetic Peptide5 (Mao et al., 2016). Treated rats showed improved locomotor activity and reduced at the level mechanical allodynia (central pain sensitization). Again lesion size was significantly reduced, astrocytic and activated macrophage and microglial responses were significantly reduced, and there was a significant sparing of neuronal cell numbers.

With respect to the inflammasome pathway specifically, as discussed above, a recent study provided evidence that spinal cord injury stimulates inflammasome activation at the injured site (Zendedel et al., 2016). The HC blocking peptides decrease levels of TNF$\alpha$ and IL- $1 \beta$, which are markers of inflammasome activation, in the wound bed 8 hours post-spinal cord injury (O'Carroll et al., 2013). This may result from a reduction in HC-mediated ATP release that triggers the inflammasome pathway. ATP release was also reduced after a weight drop spinal cord injury in a mouse model with astrocyte-specific deletion of $\mathrm{Cx} 43$ in astrocytes (Huang et al., 2012a). The blocked ATP release paralleled reduced inflammation (less astrogliosis and microglia activation), resulted in smaller lesions, and significantly improved motor recovery. Excessive ATP release from damaged tissue leads to activation of P2X7 receptors and systemic delivery of a P2X7 receptor antagonist (Brilliant Blue G) is similarly able to significantly improve outcomes, by acting downstream of the HC opening event (Wang et al., 2004; Peng et al., 2009). ATP released as result of $\mathrm{Cx} 43 \mathrm{HC}$ opening after spinal cord injury is associated with chronic pain as noted above in the Mao et al. (2016) contusion study. In animals with spinal nerve ligations, $\mathrm{Cx} 43$ siRNA downregulated $\mathrm{Cx} 43$ expression to alleviate mechanical hypersensitivity (Xu et al., 2014) and in weight drop experiments $\mathrm{Cx} 43 / \mathrm{Cx} 30$ deletions (but not $\mathrm{Cx} 30$ deletions alone) prevented heat hyperplasia and mechanical allodynia. The change was associated with reduced astrogliosis, suggesting an important role for astrocytes and specifically astrocyte $\mathrm{HC}$ opening in chronic pain (Chen et al., 2014; for further discussion of $\mathrm{Cx} 43 \mathrm{HC}$ and GJ roles chronic neuropathic pain following spinal cord injury, see also Chen et al., 2012a). In another, very comprehensive study, satellite glial cells in the cervical C6-C8 region specifically exhibited increased GFAP and Cx43 expression associated with pain after a thoracic T3 compression or transection lesion (Lee-Kubli et al., 2016). Carbenoxolone or glycyrrhizic acid blocked the pain response, as did the connexin mimetic peptides Gap27 and Gap26, but not Gap19. These authors concluded that the pain response is GJ mediated, not HC mediated, since Gap19 (a HC specific peptide blocker, see section III.B.2.a) did not reduce paw withdrawal thresholds in pain testing. However, this in is contrast to other recent reports (Chen et al., 2014; Tonkin et al., 2016) where HCs have been implicated and may reflect difficulty in delivery of the cytoplasmic tail-acting Gap19 that needs to penetrate the plasma membrane to be effective; all of the other blockers used close HCs too.

2. Gap Junctions, Hemichannels, and Stroke. Stroke is the second leading cause of death worldwide and also contributes to a large health and economic cost due to stroke-related disability, rehabilitation, and long-term care (Lo et al., 2003; Feigin et al., 2014). Stroke causes neurologic deficits arising from an acute focal injury of the CNS due to a vascular event, leading to cerebral infarction, intracerebral hemorrhage, and subarachnoid hemorrhage (Sacco et al., 2013). Ischemic stroke results after a transient or permanent occlusion of an artery supplying the brain (Sacco et al., 2013). Since ischemic stroke accounts for approximately $80 \%$ of all strokes (Thrift et al., 2001), we will focus on this condition.

Stroke affects neurons, glia, vascular cells, and extracellular matrix components, which together influence mechanisms of tissue injury and repair taking place over an acute and progressive course. Within the ischemic tissue core, low ATP levels, ionic imbalance, and metabolic failure lead to rapid cell death in minutes (Lo et al., 2003). However, outside this core there is a peri-infarct region arising due to compromised blood supply, leading to milder ischemic conditions because of some compensation by collateral perfusion (Lo et al., 2003). Within this peri-infarct region, mechanisms associated with death and repair provide strategic therapeutic targets. Much work has focused directly on prevention of, or subsequent rescue of, neurons. Astrocytes within this region contribute to neuronal survival by maintaining the extracellular ionic environment, secreting growth factors and cytokines, and 
stimulating angiogenesis (Chen and Swanson, 2003). Therefore, astrocytes provide an avenue to influence neuronal survival under ischemic injury.

During a stroke, minutes after ischemia, cellular energy is depleted and neurons and astrocytes in the ischemic core become unable to maintain transmembrane ionic gradients and die, resulting in damaged tissue, i.e., ischemia-induced cell death (Siesjö, 1992; Ginsberg, 1995). This damaged region will gradually expand to include cells in the surrounding penumbral area, which ultimately become irreversibly damaged (Hossmann, 1994). Clinical outcome of stroke and longterm prognosis are directly related to infarct size; thus larger infarcts have the worse outcome. Mechanisms that contribute to cell injury and death after ischemia are activated at the same time as endogenous neuroprotective pathways instrumental to cell survival and tissue repair. One potential contributor to ischemiainduced cell death is glutamate neurotoxicity (Chakravarthy et al., 1998; Vespa et al., 1998). Evidence indicates that ischemia-induced neurotoxicity can be the result of release of high amounts of glutamate (Rothman and Olney, 1986; Pellegrini-Giampietro et al., 1997). Cellular death associated with glutamate excitotoxicity occurs by two possible mechanisms: acute cellular swelling and late degeneration. In the first mechanism, glutamate activates ionotrophic receptors, which depolarizes the cell, resulting in cell swelling and, potentially, necrosis. Late neuronal degeneration is due to overstimulation of glutamate metabotropic receptors, resulting in massive $\mathrm{Ca}^{2+}$ influx and, ultimately, apoptosis.

$\mathrm{Cx} 43$ is the predominant GJ protein in astrocytes, and its expression is increased in reactive astrocytes in ischemic tissue (Hossain et al., 1994; Nakase et al., 2006, 2009). Cx43 membrane channels, both as GJs and $\mathrm{HCs}$, impact numerous astrocytic processes involved in brain homeostasis and tissue repair (reviewed in (Freitas-Andrade and Naus, 2016). While Cx43 expression has been shown to be directly correlated with reactive astrogliosis in brain injury (Theodoric et al., 2012); the respective contributions of GJs versus HCs were not examined. Evidence from several sources supports the importance of the astrocytic environment, particularly astrocytic networks formed by GJs, in playing a critical role in neuroprotection and neurodegeneration (Giaume et al., 2010). In contrast, enhanced $\mathrm{Cx} 43$ expression can also result in increased HCs in astrocytes, which generally is associated with increased cell injury and death (Giaume et al., 2013).

Spatial buffering of the extracellular environment is mediated through GJs between astrocytes. Thus astroglial $\mathrm{Cx} 43$ has been shown to be associated with neuroprotection and astrocyte survival in experimental stroke conditions (Naus et al., 2001; Siushansian et al., 2001; Nakase et al., 2003, 2004; Kozoriz et al., 2010, 2013; Le et al., 2014; Shinotsuka et al., 2014).
Although many studies have focused on the use of in vitro models to mimic stroke conditions, in vivo studies more closely represent the clinical situation. In this regard, transgenic and knockout mice have been extremely useful to explore the role of GJs and HCs in stroke. To specifically address the role of $\mathrm{Cx} 43$, studies were carried out on $\mathrm{Cx} 43$ knockout mice. Unfortunately, these homozygous $\mathrm{Cx} 43$ null mice $\left(\mathrm{Cx} 43^{-1-}\right)$ die shortly after birth due to a cardiac malformation (Reaume et al., 1995). In contrast, heterozygous null mice $\left(\mathrm{Cx} 43^{+/-}\right)$ survive and appear healthy, despite $50 \%$ reduction in Cx43 expression. When these heterozygous null mice were subjected to permanent middle cerebral artery occlusion ( $\mathrm{pMCAO}$ ), they exhibited a significant increase in infarct volume compared with wild-type controls (Siushansian et al., 2001), suggesting that disruption of the astrocytic gap junctional syncytium mediated by $\mathrm{Cx} 43$ is detrimental to neuronal survival in ischemic stroke conditions. However, since $\mathrm{Cx} 43$ is ubiquitously expressed, the effects observed in the heterozygous null mice may be due to other contributing systemic factors that are affected by the deletion. To address this issue, Nakase et al. (2004) investigated specifically the role of astroglial $\mathrm{Cx} 43$ in stroke using a conditional $\mathrm{Cx} 43$ knockout mouse (GFAP-Cre; $\mathrm{Cx} 43^{\mathrm{fl} / \mathrm{fl}}$ ). These $\mathrm{Cx} 43$ conditional knockout mice, lacking $\mathrm{Cx} 43$ in astrocytes, also exhibited a significant increase in stroke volume and enhanced apoptosis compared with wild-type controls when subjected to pMCAO (Nakase et al., 2004). Interestingly, a higher level of inflammation, as measured by reactive microglial cells, was also found in the $\mathrm{Cx} 43$ conditional knockout mice 4 days after pMCAO (Nakase et al., 2004). This finding highlights a possible link between astrocytic $\mathrm{Cx} 43$ expression and inflammation in ischemic conditions (Nakase et al., 2004). Several groups have shown that exposing astrocytes to inflammatory factors can induce Cx43 HC activity and affect neuronal viability in neuron/astrocyte coculture conditions (Retamal et al., 2007a; Froger et al., 2010; Orellana et al., 2011a; Boulay et al., 2016).

Various in vitro stroke models are well suited to address mechanisms regarding the roles of GJs and HCs. For example, hypoxia-reoxygenation along with elevated glucose concentration activates $\mathrm{Cx} 43 \mathrm{HCs}$, resulting in astrocyte cell death (Orellana et al., 2010). In contrast, under the same conditions astrocyte cell death was reduced by the HC blockers, Gap26, Gap27, or $\mathrm{La}^{3+}$, applied at the start of the reoxygenation period. Moreover, the p38 MAPK inhibitor, 4-(4-Fluorophenyl)2-(4-hydroxyphenyl)-5-(4-pyridyl)-1H-imidazole (SB202190), which reduces hypoxia-reoxygenation opening of $\mathrm{Cx} 43$ HCs, was also protective (Orellana et al., 2010). These results suggest ischemic conditions involving both inflammation and hypoxia synergistically promote $\mathrm{Cx} 43$ $\mathrm{HC}$ activity, contributing to both astrocyte and neuronal cell death. 
The deleterious effects of $\mathrm{Cx} 43 \mathrm{HCs}$ in stroke were further investigated in vivo using a mouse ODDD model (Kozoriz et al., 2013) (see section VII). The common external features in this syndrome, which is caused by a number of $\mathrm{Cx} 43$ mutations (Laird, 2014), include ocular, nasal, dental, and digital abnormalities, and can include neurologic deficits (Paznekas et al., 2009). Many of these $\mathrm{Cx} 43$ mutations are associated with reduced GJ formation and increased HC activity (McLachlan et al., 2005; Dobrowolski et al., 2007; Kozoriz et al., 2013). One mouse model of this disorder was created with a missense G60S point mutation (Flenniken et al., 2005); the phenotype of $\mathrm{Cx} 43^{+/ \mathrm{G} 60 \mathrm{~S}}$ mice is similar to human ODDD. Since the G60S mutation reduces the level of $\mathrm{Cx} 43$ and GJ coupling in astrocytes and also leads to increased $\mathrm{HC}$ activity, it is not surprising that these mice show a significant increase in infarct volume pMCAO (Kozoriz et al., 2013). A similar stroke study in mice expressing a C-terminal truncated $\mathrm{Cx} 43$, K258stop (Maass et al., 2004), also demonstrated an increase in stroke injury that was likewise associated with enhanced HC activation (Kozoriz et al., 2010). Taken together, these studies support the role of $\mathrm{Cx} 43$ HCs as a key focal point in stroke.

Although many studies have highlighted the role of GJs in neuroprotection, there are also several reports demonstrating that GJs also play a role in enhancing cellular injury (Cotrina et al., 1998; Lin et al., 1998; Rami et al., 2001; Nodin et al., 2005; Perez Velazquez et al., 2006). Under ischemic in vitro conditions astrocytes show reduction in coupling; however, they do not uncouple during or after a lethal injury (Cotrina et al., 1998). This raises the possibility that excess secondary intracellular messengers, such as $\mathrm{Ca}^{2+}$ and $\mathrm{IP}_{3}$, can migrate freely between dying astrocytes into healthy cells and extend the focal injury (Cotrina et al., 1998). Furthermore, administration of octanol (GJ inhibitor) led to significant reduction of $\mathrm{Cx} 43$ immunoreactivity and reduced neuronal death in the CA1-CA2 regions of ischemic animals (Rami et al., 2001). These studies suggest that reduced GJ communication may inhibit the flow of undesirable metabolites that could potentially cause neuronal damage in the peri-infarct area (Rami et al., 2001). In addition to the possible propagation of intercellular cytotoxic substances, spreading depression-like depolarizations through open GJs may also contribute to neuronal loss (Cotrina et al., 1998; Lin et al., 1998; Perez Velazquez et al., 2006). It is not understood when propagation of harmful or protective molecules takes precedence. Differences in experimental design, brain region-dependent vulnerability to ischemia, and the type of connexins and pannexins under study may explain why these GJ proteins have been observed to have either protective or destructive roles in the CNS.

Others have shown, however, that there are also reactive astrocytes with decreased $\mathrm{Cx} 43$ expression surrounding the infarct (Hossain et al., 1994; Cotrina et al., 1998). Furthermore, Cx43 expression in reactive astrocytes is spatially heterogeneous around cortical lesions induced by kainic acid excitotoxicity (Sawchuk et al., 1995; Koulakoff et al., 2008; Gangoso et al., 2012). This heterogeneity in $\mathrm{Cx} 43$ expression, in reactive astrocytes, may reflect the different astrocytic populations, with distinct molecular signatures, residing within specific brain regions (Wallraff et al., 2004). In addition, damaged ischemic neurons with varying degrees of injury may participate in regulating the surrounding astrocytic GJs. Indeed, others have shown that paracrine-secreted factors can regulate astrocyte GJs (Blomstrand et al., 2004; Koulakoff et al., 2008; Wuestefeld et al., 2012). The interplay between paracrine activation and astrocyte-type specific responses with respect to $\mathrm{Cx} 43$ expression/activity is not fully elucidated.

Various potential therapeutic interventions are currently being examined to reduce neuronal death associated with stroke. Such neuroprotective therapies include glutamate release inhibitors and receptor antagonists, $\mathrm{Ca}^{2+}$ channel blockers, GABA receptor agonists, gangliosides, neurotrophic factors, calpain inhibitors, caspase inhibitors, free radical scavengers, and immuno- and cell metabolism modulators. Very few therapeutic avenues have proven successful to treat stroke [for recent review, see Cook and Tymianski, 2011). One noteworthy success has used of a small peptide that prevents postsynaptic density protein PSD-95 from activating NMDA receptor-mediated neurotoxicity pathways (Cook et al., 2012; Hill, 2012). This establishes the validity of therapies specifically targeting interacting domains of key signaling proteins and holds promise for clinical applications, particularly related to stroke. Small biomolecules (antisense RNA, peptides) targeting GJs are in fact currently in clinical trials for healing chronic wounds and corneal injuries, providing proof of principle for these channels as therapeutic targets (Butera et al., 2009; Ormonde et al., 2012). In this context, $\mathrm{Cx} 43$ provides a substrate for therapeutic strategies targeted to astrocytes rather than neurons, based on the premise that both cell types play critical roles in stroke recovery. One such therapeutic is the peptide Gap19, which has been shown to block HCs but not GJ channels in astrocytes (Wang et al., 2013c; Abudara et al., 2014). It is envisioned that a therapeutic approach targeting both neuronal signaling pathways and astrocyte-mediated events should enhance protection after stroke.

3. Ocular Models and Disease. The link between injury and degenerative disease and loss of vascular integrity is more easily visualized in the eye. Ex vivo optic nerve and in vivo retinal injury models all demonstrate the link between inflammation and vessel leak. Isolated rat optic segments exposed to oxygen glucose deprivation for 2 hours and then placed into a 
normoxic air-liquid interface organotypic culture will swell and show an inflammatory response (increased GFAP expression and microglia activation). In addition, blood vessels become fragmented (Danesh-Meyer et al., 2008). Treatment with $\mathrm{Cx} 43$ antisense reduces edema and inflammation (both GFAP levels and number of activated microglia), and despite the absence of blood flow in the organotypic culture model, blood vessels retain greater structural integrity. In vivo, retinal ischemia is known to cause vascular leakage (Kaur et al., 2008; Abcouwer et al., 2010), with loss of vascular integrity beginning in the first few hours after ischemia but lasting for up to 2 months (Wilson et al., 1995). To establish the mechanism of vessel leak, a high intraocular pressure model of ischemia-reperfusion was used (Danesh-Meyer et al., 2012). A cannula in the anterior chamber of the eye was used to raise intraocular pressure to $120 \mathrm{mmHg}$ for 1 hour and then removed to allow reperfusion. Although the pressure was applied uniformly across the whole retina, and the whole retina is made ischemic, inflammation and $\mathrm{Cx} 43$ levels increased, starting within 2 hours, but only in small patches and adjacent to blood vessels. Evans Blue dye perfused through the animal demonstrated vascular leak in a similar pattern, starting 1-2 hours after reperfusion and indicating that vessel leak may precede inflammation, which is the result, in this case, of bloodretina barrier breaches. Confocal microscopy revealed astrocytic processes crossing the lumen of vessels, indicating that endothelial cells had burst, the authors proposing this being due to $\mathrm{HC}$ opening and an inability to osmoregulate leading to rupture. Labeling of vessels 4 hours after injury indicated the vascular bed was badly disrupted with blind endings and collapsed vessels apparent. In three separate studies, delivery of the peptidomimetic Peptide5 reduced vascular leak (up to $86 \%$ at the peak 4 hours post-reperfusion time point), reduced inflammation, and downstream (followed for up to 4 weeks later) resulted in highly significant neuronal sparing (greater than 50\% sparing in all three studies), which also has relevance for glaucoma (Chen et al., 2015b). The peptide was delivered systemically (Danesh-Meyer et al., 2012) by intravitreal injection into the eye in native form or encapsulated into slowrelease poly(lactic-coglycolic) acid nanoparticles (Chen et al., 2015d) or lipoamino acid-modified to increase stability (Chen et al., 2015c). In all three studies, a single peptide injection at the point of reperfusion was given and at a concentration expected to block HCs with minimal effect on GJ coupling. Connexin isoforms other than $\mathrm{Cx} 43$ can play a role in secondary cell death, which may offer other points of intervention. Under excitotoxic conditions in the retina (exposure to NMDA), genetic deletion of $\mathrm{Cx} 36$ increases neuronal survival by up to $50 \%$, whereas $\mathrm{Cx} 45$ deletion shows no benefit. Conversely, $\mathrm{Cx} 45$ deletion can reduce neuronal loss after an ischemic insult, but ablation of Cx36 is ineffective in this condition (Akopian et al., 2014). These latter studies, however, do not enable differentiation between GJ- or HC-mediated events.

In the retina ischemia-reperfusion studies above (Danesh-Meyer et al., 2012), breach of the blood-retina barrier appeared to be the instigating event. In a different model, a bright light damage model, inflammation appears to be the initiating factor. In this model albino rats are exposed to intense fluorescent light for 24 hours, resulting in retinal degeneration that mimics that seen in age-related macular degeneration, although at a faster rate. Photoreceptors and retinal pigment epithelium are lost, breakages appear in Bruch's membrane, and choroidal damage occurs with oxidative stress in choroidal endothelial cells (the eyes' main blood supply)( Wu et al., 2005; Marc et al., 2008). Cx43 increases after damage in parallel with inflammation, starting in the choroid first and them progressing into the retina (Guo et al., 2014). The levels of $\mathrm{Cx} 43$, remaining high over the first 2 days and only decreasing after 7 days, correlated with oxidative stress and macrophage invasion, suggesting a close relationship between $\mathrm{Cx} 43$ and the inflammatory process. In a follow up study (Guo et al., 2016), Cx43 HCs were blocked with two intravitreal injections of $\mathrm{Cx} 43$ mimetic Peptide5, one 2 hours into the 24-hour bright light period and one at the end of the bright light period. Such treatment resulted in reduced choroidal inflammation and improved functional recovery of both photoreceptors and neurons as judged from electroretinograms (mixed a-wave and mixed b-wave amplitudes, isolated rod PII and PIII amplitudes, and cone PII responses).

Another ocular disease associated with inflammation and vessel hemorrhage is diabetic retinopathy. While there are reports that $\mathrm{Cx} 43$ is downregulated in diabetes that may contribute to vascular degeneration (Tien et al., 2014, 2016), the methods used were indirect. In a recent study, $\mathrm{Cx} 43$ levels in wild-type, Akita, and Akimba mice were directly analyzed. The Akita mouse is diabetic but does not form diabetic retinopathy lesions at the ages studied. In that strain $\mathrm{Cx} 43$ levels appeared lower in the retina. In Akimba mice, however, which do develop signs of diabetic retinopathy, $\mathrm{Cx} 43$ was threefold higher in the ganglion cell layer, mainly associated with astrocytes, with increased GFAP expression in several retinal layers suggesting Müller cell activation. Importantly, there was increased blood vessel density, implying neovascularization with significantly higher $\mathrm{Cx} 43$ levels in vascular endothelial cells (Danesh-Meyer et al., 2016). Obviously, Cx43 labeling is not a marker for $\mathrm{HC}$ opening, and further interventional studies are needed. It is of note that the retinal ischemia-reperfusion model described above (DaneshMeyer et al., 2012) has also been described as a model for diabetic retinopathy, because changes observed mimic those seen in the disease (Zheng et al., 2007; Wang et al., 2011). In human donor tissues, in which 
diabetic retinopathy was confirmed with ex vivo fundus and optical coherence tomography imaging, $\mathrm{Cx} 43$ is highly upregulated compared with normal, agematched donor tissues and especially high in the endothelium of blood vessels in regions of neovascularization, the vessels that hemorrhage as the disease progresses (Danesh-Meyer et al., 2016). All of these studies support the view that connexin HC regulation may have significant treatment potential for retinal diseases such as age-related macular degeneration, diabetic retinopathy, and macular edema. This may either be through the maintenance of vascular integrity or by damping down the inflammatory process, especially the inflammasome pathway, which is known to be a feature of these diseases (Tseng et al., 2013; Qi et al., 2014; Chi et al., 2015; Gao et al., 2015).

4. Neurodegenerative Brain Disease. Although neurodegenerative brain diseases are not strictly vascular diseases, several of them are associated with inflammation and loss of vascular integrity, and we therefore briefly discuss the role of connexins in these diseases. Alzheimer's disease, although noted for the accumulation of amyloid- $\beta$ beta plaques and neurofibrillary tangles, is characterized by chronic brain inflammation and vascular dropout (Farkas and Luiten, 2001). In animal models, controlling leukocyte-endothelial interactions inhibits both amyloid deposition and reduces memory loss in those animals, suggesting that cerebrovascular disease is upstream of amyloid deposition and the formation of neurofibrillary tangles (Zenaro et al., 2016). Indeed vascular dysfunction diminishes brain capillary flow, reducing amyloid- $\beta$ clearance and leading to neuronal dysfunction (Janota et al., 2016), and mutations known to provoke inflammatory reactions are proposed to cause microvasculature damage leading to dementia (Marchesi, 2016). Once formed though, amyloid- $\beta$ may further exacerbate injury by enhancing connexin43 expression (Nagy et al., 1996). In Parkinson's disease, another chronic inflammatory disorder, there is small vessel dropout in brain regions affected by the disease (Guan et al., 2013), and in Lewy body dementia, reduced microvessel density, rather than vasoconstriction, is said to be the cause of reduced occipital blood flow (Miners et al., 2014). Furthermore, because the chronic inflammation cannot be contained, there is a link between diseases, with circulating inflammatory cytokines triggering disease elsewhere (Green and Nicholson, 2008). In a recent 2016 example (Yeh et al., 2016), the Parkinson's disease-free survival rate was significantly lower for patients who had spinal cord injury than for uninjured patients, with almost double the rate of Parkinson's onset in the spinal cord injury group.

The link between connexin channels and neurodegenerative disease is very strong, especially connexin HCs but also pannexin channels in inflammatory responses of glia and neurons (Orellana et al., 2011b,
2012; Bennett et al., 2012; Retamal, 2014). GJ inhibitors, and in particular connexin HC blockers, suppress excessive microglial glutamate release and have improved outcomes in animal models of multiple sclerosis, amyotrophic lateral sclerosis, and Alzheimer's disease (Takeuchi et al., 2011; Takeuchi and Suzumura, 2014; Yi et al., 2016). HC opening in astrocytes and microglia appears to initiate a "vicious cycle" of excessive ATP release underlying cerebral palsy resulting from preterm ischemia (Mallard et al., 2014), and the term HC-mediated "vicious cycle" emerges again in a murine model of Alzheimer's disease (Yi et al., 2016). In this very thorough study, the expression of connexins was shown to be upregulated in both human donor brain tissue and in an APP/PS1 mouse model of Alzheimer's disease. In acute hippocampal slices of tissue containing amyloid plaques, GJ communication was not altered (assessed using fluorescence recovery after photobleaching) but HCs were activated in astrocytes (assessed using EtBr dye uptake). A number of GJ (CBX, Gap26), HC $\left(\mathrm{La}^{3+}\right)$, or pannexin channel $\left({ }^{10}\right.$ Panx 1 , probenecid) blockers were used, as well as astroglial targeted $\mathrm{Cx} 43$ gene knockout. A minor pannexin channel component was observed in astrocytes immediately contacting the amyloid plaques, but connexin HC opening was widespread, leading to the release of ATP and glutamate. This maintained "a high calcium level in astrocytes placing them in the center of a vicious circle." With targeted knockout of $\mathrm{Cx} 43$ in astrocytes, gliotransmitter release was diminished, reducing oxidative stress and damage to hippocampal neurons associated with amyloid plaques. In two other transgenic models, mice carrying mutated human superoxide dismutase 1 as an amyotrophic lateral sclerosis model and double transgenic mice expressing mutated human amyloid precursor protein and presenilin 1 as an Alzheimer's disease model, a modified glycyrrhetinic acid connexin channel blocker inhibited excessive glutamate release from activated microglia (Takeuchi et al., 2011). Those authors too concluded that HC block may be effective in preventing microgliamediated neuronal death in neurodegenerative diseases.

In vitro models have produced significant information on GJ and HC roles in cerebrovascular and retinovascular disease, although results can appear conflicting owing to the diversity of models, cell types used, time course, and dosing. Many of these may not always have relevance to the in vivo situation during an acute injury phase or in chronic disease. Nonetheless, patterns are emerging and increasing numbers of animal models, with improved channel blockers and knowledge of their pharmacodynamics available, are leading to consistency in demonstrating pathologic opening of connexin $\mathrm{HCs}$ and their role in inflammation, lesion spread, edema, loss of vascular integrity, and neuronal degeneration. More importantly, HC opening is 
increasingly associated with perpetuation of inflammation, in particular the inflammasome pathway, in chronic disease. This has already been implicated in a remarkable number of CNS cerebrovascular and retinovascular indications including trauma, such as spinal cord injury or stroke, and chronic diseases including Alzheimer's, Parkinson's, amyotrophic lateral sclerosis, multiple sclerosis, diabetic retinopathy, macular edema, age-related macular degeneration, and chronic pain. While the field could still benefit from more specific reagents, a number of those existing are showing significantly improved outcomes in animal models where the therapeutic target is identified. Hopefully clinical advances can now be made for these indications where there is an unmet need for intervention.

\section{Concluding Remarks}

In recent years we have seen the appearance of a number of interesting peptides such as L2, Gap19, Peptide5, AAP10 and its D-amino acid version rotigaptide (ZP123), and $\alpha \mathrm{CT} 1$ composed of $\mathrm{Cx} 43$ CT9 peptide fused to an antennapedia membrane translocation motif. The mother peptide L2 and the pharmacophorebased peptidomimetic molecule ZP2519 target the $\mathrm{Cx} 43$ $\mathrm{CT}$ and prevent $\mathrm{Cx} 43$ GJ closure at low $\mathrm{pH}$, as occurs in ischemia. Thus, ZP2519 has potential translational value for preventing postischemic GJ closure and arrhythmias. Interestingly, L2 and the L2 subdomain peptide Gap19 are blockers of Cx43-based HCs. L2 thus combines two interesting actions: it prevents acidification-induced GJ closure and inhibits HC opening. It would be interesting to test whether ZP2519 retains the $\mathrm{HC}$-inhibiting properties of the mother peptide L2. AAP10 and ZP123 promote coupling via Cx43- and Cx45-based GJs and are interesting substances for the treatment of ischemia-linked arrhythmias. A phase II clinical trial investigating the safety and tolerability of ZP123 in patients suffering from unstable angina or myocardial infarction has been initiated (Kjølbye et al., 2007), but despite promising results, it was terminated (Zhang and Xiang, 2009). $\alpha \mathrm{CT} 1$ peptide has substantial promise because it promotes GJs not by acting on the channels per se but by promoting the incorporation of $\mathrm{Cx} 43 \mathrm{HCs}$ in $\mathrm{GJ}$ plaques.

In terms of vascular disease, animal studies have revealed important and diverse contributions of the vascular connexins $\mathrm{Cx} 37, \mathrm{Cx} 40$, and $\mathrm{Cx} 43$ to atherogenesis, with $\mathrm{Cx} 43$ having an overall atherogenic effect while $\mathrm{Cx} 40$ protects against atherosclerosis and loss of Cx37 promotes atherosclerosis. Several human Cx37 SNPs have been identified, and those affecting connexin GJ or HC function appear to link to cardiovascular disease risk. Mice cardiac ischemia-reperfusion studies have unveiled a protective role of vascular endothelial $\mathrm{Cx} 40$, which limits neutrophil infiltration and inflammation in the reperfusion phase. In the heart, ischemic preconditioning and the role of mitochondrial $\mathrm{Cx} 43$ therein remains an interesting field to be further explored toward development of peptides and drugs that target this intracellular connexin pool. In addition to GJs and plasma membrane $\mathrm{HCs}$, mitochondrial $\mathrm{Cx} 43$ should be considered as a third level for pharmacotherapeutic intervention. Cardiac fibrosis appears to involve ATP release through $\mathrm{Cx} 43-$ and $\mathrm{Cx} 45$ based HCs in cardiac fibroblasts (Lu et al., 2012a) and fibroblast-cardiomyocyte GJ coupling may influence electrical signaling (Ongstad and Kohl, 2016), putting fibroblast connexins as another potential target in view. In terms of cardiac arrhythmias, alterations in the distribution and the abundance of GJs composed of $\mathrm{Cx} 40$ and $\mathrm{Cx} 43$ resulting from heart failure or other cardiac pathologies may lead to arrhythmogenesis. It remains to be seen whether HCs may contribute as an additional arrhythmogenic channel as recently suggested based on work with Gap19 and other peptides (González et al., 2015).

In cerebrovascular and retinovascular disease, HC-blocking peptides such as Peptide5 come up as interesting tools to reduce inflammation and preserve vascular integrity. Connexins in the vascular endothelium are intrinsically an utmost interesting target, because blood cells and endothelial cells are the first line to welcome a drug after absorption in the gastrointestinal tract. Obviously, we need more and better tools to target specific connexins and specific channels: GJs or HCs. These tools are urgently needed to understand better and distinguish HC functions from those of GJs. However, in terms of therapeutic application, the most specific tools not always make the best drugs. Spinal cord injury, stroke, diabetic retinopathy, macular edema, and age-related macular degeneration come up as diseases where novel connexin-targeting drugs would be very much welcome. There is currently a growing interest in connexin-targeting drugs for applications to skin, wound healing, and ocular disease (Ghatnekar et al., 2009; Grupcheva et al., 2012; Grek et al., 2015; Becker et al., 2016; reviewed in DaneshMeyer et al., 2016; Naus and Giaume, 2016). Additionally, some existing drugs are repurposed to exploit their effects on connexins in a combinatorial approach, as, e.g., in the combination of modafinil with the GJ-inhibiting properties of flecainide to treat narcolepsy (Duchêne et al., 2016). Clearly, these examples just mark the start of a rapidly expanding interest in connexins as a potential pharmacotherapeutic target. At this stage, two important points on the agenda to get the field forward are 1) to better understand why EL interactions upon $\mathrm{HC}$ docking and formation of a GJ opens HCs, whereas applying peptides mimicking crucial EL domains closes unapposed HCs, and 2) why Cx43 loop-tail (CT-CL) interactions have a completely opposite effect on HCs compared with GJs. Increased 
insights in these important questions will be helpful in designing novel molecules that target connexin channels from the outside or inside of the cell.

\section{Conflict of Interest}

\section{Acknowledgments}

We thank Dr. Anna Pfenniger and Dr. Sandrine Morel for help with the art work of Figs. 5 and 6, Layo Alaga for generation of Fig. 8, and Dr. Yeri Kim (University of Auckland) for help in assembling Fig. 9. Figure 1 was constructed based on a backbone generated by the Protter tool (Omasits et al., 2014).

D. W. Laird holds a Canada Research Chair in Gap Junctions and Disease, C. C. Naus holds a Canada Research Chair, and C. R. Green holds the W\&B Hadden Chair in Ophthalmology and Translational Vision Research and acknowledges the generosity of Wendy and Bruce Hadden in funding this position.

We apologize for any references we might have overlooked.

\section{Authorship Contributions}

Wrote or contributed to the writing of the manuscript: Leybaert, Lampe, Dhein, Kwak, Ferdinandy, Beyer, Laird, Naus, Green, Schulz.

\section{References}

Abascal F and Zardoya R (2013) Evolutionary analyses of gap junction protein families. Biochim Biophys Acta 1828:4-14.

Abcouwer SF, Lin CM, Wolpert EB, Shanmugam S, Schaefer EW, Freeman WM, Barber AJ, and Antonetti DA (2010) Effects of ischemic preconditioning and bevacizumab on apoptosis and vascular permeability following retinal ischemiareperfusion injury. Invest Ophthalmol Vis Sci 51:5920-5933.

Abrams CK and Scherer SS (2012) Gap junctions in inherited human disorders of the central nervous system. Biochim Biophys Acta 1818:2030-2047.

Abudara V, Bechberger J, Freitas-Andrade M, De Bock M, Wang N, Bultynck G, Naus CC, Leybaert L, and Giaume C (2014) The connexin43 mimetic peptide Gap19 inhibits hemichannels without altering gap junctional communication in astrocytes. Front Cell Neurosci 8:306.

Agullo-Pascual E, Cerrone M, and Delmar M (2014a) Arrhythmogenic cardiomyopathy and Brugada syndrome: diseases of the connexome. FEBS Lett $\mathbf{5 8 8}$ 1322-1330.

Agullo-Pascual E, Lin X, Leo-Macias A, Zhang M, Liang FX, Li Z, Pfenniger A Lübkemeier I, Keegan S, Fenyö D, et al. (2014b) Super-resolution imaging reveals that loss of the C-terminus of connexin 43 limits microtubule plus-end capture and NaV1.5 localization at the intercalated disc. Cardiovasc Res 104:371-381.

Ahlquist JA, Franklyn JA, Ramsden DB, and Sheppard MC (1989) The influence of dexamethasone on serum thyrotrophin and thyrotrophin synthesis in the rat. Mol Cell Endocrinol 64:55-61.

Ai X and Pogwizd SM (2005) Connexin 43 downregulation and dephosphorylation in nonischemic heart failure is associated with enhanced colocalized protein phosphatase type 2A. Circ Res 96:54-63.

Ai X, Zhao W, and Pogwizd SM (2010) Connexin43 knockdown or overexpression modulates cell coupling in control and failing rabbit left ventricular myocytes. Cardiovasc Res 85:751-762.

Ai X, Jiang A, Ke Y, Solaro RJ, and Pogwizd SM (2011) Enhanced activation of p21activated kinase 1 in heart failure contributes to dephosphorylation of connexin 43. Cardiovasc Res 92:106-114.

Ai Z, Fischer A, Spray DC, Brown AM, and Fishman GI (2000) Wnt-1 regulation of connexin43 in cardiac myocytes. J Clin Invest 105:161-171.

Akar FG, Spragg DD, Tunin RS, Kass DA, and Tomaselli GF (2004) Mechanisms underlying conduction slowing and arrhythmogenesis in nonischemic dilated cardiomyopathy. Circ Res 95:717-725.

Akopian A, Atlasz T, Pan F, Wong S, Zhang Y, Völgyi B, Paul DL, and Bloomfield SA (2014) Gap junction-mediated death of retinal neurons is connexin and insult specific: a potential target for neuroprotection. $J$ Neurosci 34:10582-10591.

Alesutan I, Voelkl J, Stöckigt F, Mia S, Feger M, Primessnig U, Sopjani M, Munoz C, Borst O, Gawaz M, et al. (2015) AMP-activated protein kinase $\alpha 1$ regulates cardiac gap junction protein connexin 43 and electrical remodeling following pressure overload. Cell Physiol Biochem 35:406-418.

Alev C, Urschel S, Sonntag S, Zoidl G, Fort AG, Hoher T, Matsubara M, Willecke K, Spray DC, and Dermietzel R (2008) The neuronal connexin36 interacts with and is phosphorylated by CaMKII in a way similar to CaMKII interaction with glutamate receptors. Proc Natl Acad Sci USA 105:20964-20969.

Alexander DB and Goldberg GS (2003) Transfer of biologically important molecules between cells through gap junction channels. Curr Med Chem 10:2045-2058.

Allen MJ, Gemel J, Beyer EC, and Lal R (2011) Atomic force microscopy of Connexin40 gap junction hemichannels reveals calcium-dependent three-dimensional molecular topography and open-closed conformations of both the extracellular and cytoplasmic faces. J Biol Chem 286:22139-22146.

Allessie MA, Bonke FI, and Schopman FJ (1977) Circus movement in rabbit atrial muscle as a mechanism of tachycardia. III. The "leading circle" concept: a new model of circus movement in cardiac tissue without the involvement of an anatomical obstacle. Circ Res 41:9-18.

Allessie MA, Boyden PA, Camm AJ, Kléber AG, Lab MJ, Legato MJ, Rosen MR Schwartz PJ, Spooner PM, Van Wagoner DR, et al. (2001) Pathophysiology and prevention of atrial fibrillation. Circulation 103:769-777.

Amagaya S, Sugishita E, Ogihara Y, Ogawa S, Okada K, and Aizawa T (1984) Comparative studies of the stereoisomers of glycyrrhetinic acid on antiinflammatory activities. J Pharmacobiodyn 7:923-928.

Ambrosi C, Gassmann O, Pranskevich JN, Boassa D, Smock A, Wang J, Dahl G, Steinem C, and Sosinsky GE (2010) Pannexin1 and Pannexin2 channels show quaternary similarities to connexons and different oligomerization numbers from each other. J Biol Chem 285:24420-24431.

Ambrosi C, Ren C, Spagnol G, Cavin G, Cone A, Grintsevich EE, Sosinsky GE, and Sorgen PL (2016) Connexin43 forms supramolecular complexes through non-overlapping binding sites for drebrin, tubulin, and ZO-1. PLoS One 11: $\mathrm{e} 0157073$.

Anderson MF, Blomstrand F, Blomstrand C, Eriksson PS, and Nilsson M (2003) Astrocytes and stroke: networking for survival? Neurochem Res 28:293-305.

Angelillo-Scherrer A, Fontana P, Burnier L, Roth I, Sugamele R, Brisset A, Morel S, Nolli S, Sutter E, Chassot A, et al. (2011) Connexin 37 limits thrombus propensity by downregulating platelet reactivity. Circulation 124:930-939.

Anna Z, Angela S, Barbara B, Jana R, Tamara B, Csilla V, Victor D, Oleksiy M, and Narcisa T (2014) Heart-protective effect of n-3 PUFA demonstrated in a rat model of diabetic cardiomyopathy. Mol Cell Biochem 389:219-227.

Anselmi F, Hernandez VH, Crispino G, Seydel A, Ortolano S, Roper SD, Kessaris N, Richardson W, Rickheit G, Filippov MA, et al. (2008) ATP release through connexin hemichannels and gap junction transfer of second messengers propagate $\mathrm{Ca}^{2+}$ signals across the inner ear. Proc Natl Acad Sci USA 105:18770-18775.

Anumonwo JM, Taffet SM, Gu H, Chanson M, Moreno AP, and Delmar M (2001) The carboxyl terminal domain regulates the unitary conductance and voltage dependence of connexin40 gap junction channels. Circ Res 88:666-673.

Aonuma S, Kohama Y, Akai K, Komiyama Y, Nakajima S, Wakabayashi M, and Makino T (1980) Studies on heart. XIX. Isolation of an atrial peptide that improves the rhythmicity of cultured myocardial cell clusters. Chem Pharm Bull (Tokyo) 28:3332-3339.

Archard HO and Denys FR (1979) Development of annular gap junctions in guinea pig epithelia. J Oral Pathol 8:187-197.

Atkinson MM, Anderson SK, and Sheridan JD (1986) Modification of gap junctions in cells transformed by a temperature-sensitive mutant of Rous sarcoma virus. $J$ Membr Biol 91:53-64.

Atkinson MM, Menko AS, Johnson RG, Sheppard JR, and Sheridan JD (1981) Rapid and reversible reduction of junctional permeability in cells infected with a temperature-sensitive mutant of avian sarcoma virus. J Cell Biol 91: $573-578$

Atkinson MM, Lampe PD, Lin HH, Kollander R, Li XR, and Kiang DT (1995) Cyclic AMP modifies the cellular distribution of connexin 43 and induces a persistent increase in the junctional permeability of mouse mammary tumor cells. $J$ Cell $S c i$ 108:3079-3090.

Auth T, Schlüter S, Urschel S, Kussmann P, Sonntag S, Höher T, Kreuzberg MM, Dobrowolski R, and Willecke K (2009) The TSG101 protein binds to connexins and is involved in connexin degradation. Exp Cell Res 315:1053-1062.

Avendaño BC, Montero TD, Chávez CE, von Bernhardi R, and Orellana JA (2015) Prenatal exposure to inflammatory conditions increases $\mathrm{Cx} 43$ and Panx1 unopposed channel opening and activation of astrocytes in the offspring effect on neuronal survival. Glia.63:2058-2072.

Axelsen LN, Stahlhut M, Mohammed S, Larsen BD, Nielsen MS, Holstein-Rathlou NH, Andersen S, Jensen ON, Hennan JK, and Kjølbye AL (2006) Identification of ischemia-regulated phosphorylation sites in connexin43: a possible target for the antiarrhythmic peptide analogue rotigaptide (ZP123). J Mol Cell Cardiol 40 $790-798$.

Axelsen LN, Calloe K, Braunstein TH, Riemann M, Hofgaard JP, Liang B, Jensen $\mathrm{CF}$, Olsen KB, Bartels ED, Baandrup U, et al. (2015) Diet-induced pre-diabetes slows cardiac conductance and promotes arrhythmogenesis. Cardiovasc Diabetol 14:87.

Azarnia R, Reddy S, Kmiecik TE, Shalloway D, and Loewenstein WR (1988) The cellular src gene product regulates junctional cell-to-cell communication. Science 239:398-401.

Bačová B, Radošinská J, Viczenczová C, Knezl V, Dosenko V, Beňova T, Navarová J, Gonçalvesová E, van Rooyen J, Weismann P, et al. (2012) Up-regulation of myocardial connexin-43 in spontaneously hypertensive rats fed red palm oil is most likely implicated in its anti-arrhythmic effects. Can $J$ Physiol Pharmacol 90: $1235-1245$

Bagwe S, Berenfeld O, Vaidya D, Morley GE, and Jalife J (2005) Altered right atria excitation and propagation in connexin40 knockout mice. Circulation 112: $2245-2253$

Baker TK, Kwiatkowski AP, Madhukar BV, and Klaunig JE (1995) Inhibition of gap junctional intercellular communication by 2,3,7,8-tetrachlorodibenzo-p-dioxin (TCDD) in rat hepatocytes. Carcinogenesis 16:2321-2326.

Balda MS and Matter K (2000) The tight junction protein ZO-1 and an interacting transcription factor regulate ErbB-2 expression. EMBO J 19:2024-2033.

Ballif BA, Carey GR, Sunyaev SR, and Gygi SP (2008) Large-scale identification and evolution indexing of tyrosine phosphorylation sites from murine brain. J Proteome Res 7:311-318.

Bao X, Reuss L, and Altenberg GA (2004) Regulation of purified and reconstituted connexin 43 hemichannels by protein kinase C-mediated phosphorylation of Serine 368. J Biol Chem 279:20058-20066.

Bao X, Lee SC, Reuss L, and Altenberg GA (2007) Change in permeant size selectivity by phosphorylation of connexin 43 gap-junctional hemichannels by PKC. Proc Natl Acad Sci USA 104:4919-4924.

Bargiello TA, Tang Q, Oh S, and Kwon T (2012) Voltage-dependent conformational changes in connexin channels. Biochim Biophys Acta 1818:1807-1822. 
Bargiotas P, Krenz A, Hormuzdi SG, Ridder DA, Herb A, Barakat W, Penuela S, von Engelhardt J, Monyer H, and Schwaninger M (2011) Pannexins in ischemiainduced neurodegeneration. Proc Natl Acad Sci USA 108:20772-20777.

Barker RJ, Price RL, and Gourdie RG (2002) Increased association of ZO-1 with connexin43 during remodeling of cardiac gap junctions. Circ Res 90:317-324.

Baron L, Gombault A, Fanny M, Villeret B, Savigny F, Guillou N, Panek C, Le Bert M, Lagente V, Rassendren F, et al. (2015) The NLRP3 inflammasome is activated by nanoparticles through ATP, ADP and adenosine. Cell Death Dis 6:e1629.

Basheer WA, Harris BS, Mentrup HL, Abreha M, Thames EL, Lea JB, Swing DA, Copeland NG, Jenkins NA, Price RL, et al. (2015) Cardiomyocyte-specific overexpression of the ubiquitin ligase Wwp1 contributes to reduction in Connexin 43 and arrhythmogenesis. J Mol Cell Cardiol 88:1-13.

Bastiaanse EM, Jongsma HJ, van der Laarse A, and Takens-Kwak BR (1993) Heptanol-induced decrease in cardiac gap junctional conductance is mediated by a decrease in the fluidity of membranous cholesterol-rich domains. J Membr Biol 136:135-145.

Batra N, Riquelme MA, Burra S, and Jiang JX (2014) 14-3-30 facilitates plasma membrane delivery and function of mechanosensitive connexin 43 hemichannels. $J$ Cell Sci 127:137-146.

Baum JR, Dolmatova E, Tan A, and Duffy HS (2012) Omega 3 fatty acid inhibition of inflammatory cytokine-mediated connexin 43 regulation in the heart. Front Physiol 3:272.

Beahm DL and Hall JE (2002) Hemichannel and junctional properties of connexin 50 Biophys J 82:2016-2031.

Beardslee MA, Laing JG, Beyer EC, and Saffitz JE (1998) Rapid turnover of connexin43 in the adult rat heart. Circ Res 83:629-635.

Beardslee MA, Lerner DL, Tadros PN, Laing JG, Beyer EC, Yamada KA, Kléber AG, Schuessler RB, and Saffitz JE (2000) Dephosphorylation and intracellular redistribution of ventricular connexin43 during electrical uncoupling induced by ischemia. Circ Res 87:656-662.

Beaumont M and Maccaferri G (2011) Is connexin36 critical for GABAergic hypersynchronization in the hippocampus? J Physiol 589:1663-1680.

Becker DL, Phillips AR, Duft BJ, Kim Y, and Green CR (2016) Translating connexin biology into therapeutics. Semin Cell Dev Biol 50:49-58.

Bedner P, Niessen H, Odermatt B, Kretz M, Willecke K, and Harz H (2006) Selective permeability of different connexin channels to the second messenger cyclic AMP. $J$ Biol Chem 281:6673-6681.

Bedner P, Niessen H, Odermatt B, Willecke K, and Harz H (2003) A method to determine the relative cAMP permeability of connexin channels. Exp Cell Res 291: $25-35$

Begandt D, Good ME, Keller AS, DeLalio LJ, Rowley C, Isakson BE, and Figueroa XF (2017) Pannexin channel and connexin hemichannel expression in vascular function and inflammation. BMC Cell Biol 18 (Suppl 1):2.

Bejarano E, Girao H, Yuste A, Patel B, Marques C, Spray DC, Pereira P, and Cuervo AM (2012) Autophagy modulates dynamics of connexins at the plasma membrane in a ubiquitin-dependent manner. Mol Biol Cell 23:2156-2169.

Bejarano E, Yuste A, Patel B, Stout RF Jr, Spray DC, and Cuervo AM (2014) Connexins modulate autophagosome biogenesis. Nat Cell Biol 16:401-414.

Belluardo N, Mudò G, Trovato-Salinaro A, Le Gurun S, Charollais A, Serre-Beinier V, Amato G, Haefliger JA, Meda P, and Condorelli DF (2000) Expression of connexin36 in the adult and developing rat brain. Brain Res 865:121-138.

Belluardo N, White TW, Srinivas M, Trovato-Salinaro A, Ripps H, Mudò G, Bruzzone $\mathrm{R}$, and Condorelli DF (2001) Identification and functional expression of $\mathrm{HCx} 31.9$, a novel gap junction gene. Cell Commun Adhes 8:173-178.

Bennett MV (2011) Not what you thought: how $\mathrm{H}^{+}$ions combine with taurine or other aminosulfonates to close Cx26 channels. J Gen Physiol 138:377-380.

Bennett MV, Contreras JE, Bukauskas FF, and Sáez JC (2003) New roles for astrocytes: gap junction hemichannels have something to communicate. Trends Neurosci 26:610-617.

Bennett MV, Garré JM, Orellana JA, Bukauskas FF, Nedergaard M, and Sáez JC (2012) Connexin and pannexin hemichannels in inflammatory responses of glia and neurons. Brain Res 1487:3-15.

Benova T, Viczenczova C, Radosinska J, Bacova B, Knezl V, Dosenko V, Weismann P, Zeman M, Navarova J, and Tribulova N (2013) Melatonin attenuates hypertensionrelated proarrhythmic myocardial maladaptation of connexin-43 and propensity of the heart to lethal arrhythmias. Can J Physiol Pharmacol 91:633-639.

Berthoud VM, Bassnett S, and Beyer EC (1999) Cultured chicken embryo lens cells resemble differentiating fiber cells in vivo and contain two kinetic pools of connexin56. Exp Eye Res 68:475-484.

Berthoud VM, Beyer EC, and Seul KH (2000) Peptide inhibitors of intercellular communication. Am J Physiol Lung Cell Mol Physiol 279:L619-L622.

Berthoud VM, Minogue PJ, Laing JG, and Beyer EC (2004) Pathways for degradation of connexins and gap junctions. Cardiovasc Res 62:256-267.

Betanzos A, Huerta M, Lopez-Bayghen E, Azuara E, Amerena J, and GonzálezMariscal L (2004) The tight junction protein ZO-2 associates with Jun, Fos and C/EBP transcription factors in epithelial cells. Exp Cell Res 292:51-66.

Beyer EC and Berthoud VM (2002) Gap junction synthesis and degradation as therapeutic targets. Curr Drug Targets 3:409-416.

Beyer EC and Berthoud VM (2009) The family of connexin genes, in Connexins: A Guide (Harris A and Locke D eds) pp 3-26, Humana Press, New York.

Beyer EC, Paul DL, and Goodenough DA (1987) Connexin43: a protein from rat heart homologous to a gap junction protein from liver. J Cell Biol 105:2621-2629.

Beyer EC, Paul DL, and Goodenough DA (1990) Connexin family of gap junction proteins. J Membr Biol 116:187-194.

Beyer EC, Lin X, and Veenstra RD (2013) Interfering amino terminal peptides and functional implications for heteromeric gap junction formation. Front Pharmacol 4

Billaud M, Dahan D, Marthan R, Savineau JP, and Guibert C (2011) Role of the gap junctions in the contractile response to agonists in pulmonary artery from two rat models of pulmonary hypertension. Respir Res 12:30.
Billaud M, Sandilos JK, and Isakson BE (2012) Pannexin 1 in the regulation of vascular tone. Trends Cardiovasc Med 22:68-72.

Blackburn JP, Peters NS, Yeh HI, Rothery S, Green CR, and Severs NJ (1995) Upregulation of connexin43 gap junctions during early stages of human coronary atherosclerosis. Arterioscler Thromb Vasc Biol 15:1219-1228.

Blomstrand F, Venance L, Sirén AL, Ezan P, Hanse E, Glowinski J, Ehrenreich H, and Giaume C (2004) Endothelins regulate astrocyte gap junctions in rat hippocampal slices. Eur J Neurosci 19:1005-1015.

Bodendiek SB and Raman G (2010) Connexin modulators and their potential targets under the magnifying glass. Curr Med Chem 17:4191-4230.

Bodendiek SB, Rubinos C, Trelles MP, Coleman N, Jenkins DP, Wulff H, and Srinivas M (2012) Triarylmethanes, a new class of cx50 inhibitors. Front Pharmacol 3:106.

Boengler K, Dodoni G, Rodriguez-Sinovas A, Cabestrero A, Ruiz-Meana M, Gres P, Konietzka I, Lopez-Iglesias C, Garcia-Dorado D, Di Lisa F, et al. (2005) Connexin 43 in cardiomyocyte mitochondria and its increase by ischemic preconditioning. Cardiovasc Res 67:234-244.

Boengler K, Konietzka I, Buechert A, Heinen Y, Garcia-Dorado D, Heusch G, and Schulz R (2007) Loss of ischemic preconditioning's cardioprotection in aged mouse hearts is associated with reduced gap junctional and mitochondrial levels of connexin 43. Am J Physiol Heart Circ Physiol 292:H1764-H1769.

Boengler K, Stahlhofen S, van de Sand A, Gres P, Ruiz-Meana M, Garcia-Dorado D, Heusch G, and Schulz R (2009) Presence of connexin 43 in subsarcolemmal, but not in interfibrillar cardiomyocyte mitochondria. Basic Res Cardiol 104:141-147.

Boengler K, Heusch G, and Schulz R (2011) Nuclear-encoded mitochondrial proteins and their role in cardioprotection. Biochim Biophys Acta 1813:1286-1294

Boengler K, Ruiz-Meana M, Gent S, Ungefug E, Soetkamp D, Miro-Casas E, Cabestrero A, Fernandez-Sanz C, Semenzato M, Di Lisa F, et al. (2012) Mitochondrial connexin 43 impacts on respiratory complex I activity and mitochondrial oxygen consumption. J Cell Mol Med 16:1649-1655

Boengler K, Bulic M, Schreckenberg R, Schluter KD, and Schulz R (2017) The gap junction modifier ZP1609 decreases cardiomyocyte hypercontracture following ischaemia/reperfusion independent from mitochondrial connexin 43. $\mathrm{Br} J$ Pharmacol 174:2060-2073.

Boitano S and Evans WH (2000) Connexin mimetic peptides reversibly inhibit $\mathrm{Ca}^{(2+}$ signaling through gap junctions in airway cells. Am J Physiol Lung Cell Mol Physiol 279:L623-L630.

Bol M, Wang N, De Bock M, Wacquier B, Decrock E, Gadicherla A, Decaluwe K, Vanheel B, van Rijen HV, Krysko DV, et al. (2016) At the cross-point of connexins, calcium and ATP: blocking hemichannels inhibits vasoconstriction of rat small mesenteric arteries. Cardiovasc Res 113:195-206.

Bolon ML, Peng T, Kidder GM, and Tyml K (2008) Lipopolysaccharide plus hypoxia and reoxygenation synergistically reduce electrical coupling between microvascular endothelial cells by dephosphorylating connexin40. J Cell Physiol 217:350-359.

Bonda TA, Szynaka B, Sokołowska M, Dziemidowicz M, Winnicka MM, Chyczewski L, and Kamiński KA (2016) Remodeling of the intercalated disc related to aging in the mouse heart. $J$ Cardiol 68:261-268.

Bonnette PC, Robinson BS, Silva JC, Stokes MP, Brosius AD, Baumann A, and Buckbinder L (2010) Phosphoproteomic characterization of PYK2 signaling pathways involved in osteogenesis. J Proteomics 73:1306-1320.

Boulay AC, Cisternino S, and Cohen-Salmon M (2016) Immunoregulation at the gliovascular unit in the healthy brain: a focus on connexin 43. Brain Behav Immun $\mathbf{5 6}: 1-9$

Bouvier D, Spagnol G, Chenavas S, Kieken F, Vitrac H, Brownell S, Kellezi A, Forge $\mathrm{V}$, and Sorgen PL (2009) Characterization of the structure and intermolecular interactions between the connexin 40 and connexin 43 carboxyl-terminal and cytoplasmic loop domains. J Biol Chem 284:34257-34271.

Boyett MR, Inada S, Yoo S, Li J, Liu J, Tellez J, Greener ID, Honjo H, Billeter R, Lei M, et al. (2006) Connexins in the sinoatrial and atrioventricular nodes. Adv Cardiol 42:175-197.

Brandenburger T, Huhn R, Galas A, Pannen BH, Keitel V, Barthel F, Bauer I, and Heinen A (2014) Remote ischemic preconditioning preserves connexin 43 phosphorylation in the rat heart in vivo. J Transl Med 12:228.

Brightman MW and Reese TS (1969) Junctions between intimately apposed cell membranes in the vertebrate brain. $J$ Cell Biol 40:648-677.

Brink PR, Valiunas V, Wang HZ, Zhao W, Davies K, and Christ GJ (2006) Experimental diabetes alters connexin43 derived gap junction permeability in short-term cultures of rat corporeal vascular smooth muscle cells. J Urol 175:381-386.

Brink PR, Valiunas V, Gordon C, Rosen MR, and Cohen IS (2012) Can gap junctions deliver? Biochim Biophys Acta 1818:2076-2081.

Brown TR, Krogh-Madsen T, and Christini DJ (2015) Computational approaches to understanding the role of fibroblast-myocyte interactions in cardiac arrhythmogenesis. BioMed Res Int 2015:465714.

Bruzzone S, Guida L, Zocchi E, Franco L, and De Flora A (2001) Connexin 43 hem channels mediate $\mathrm{Ca}^{2+}$-regulated transmembrane $\mathrm{NAD}^{+}$fluxes in intact cells. FASEB J 15:10-12.

Bruzzone R, Hormuzdi SG, Barbe MT, Herb A, and Monyer H (2003) Pannexins, a family of gap junction proteins expressed in brain. Proc Natl Acad Sci USA 100: 13644-13649.

Bruzzone R, Barbe MT, Jakob NJ, and Monyer H (2005) Pharmacological properties of homomeric and heteromeric pannexin hemichannels expressed in Xenopus oocytes. J Neurochem 92:1033-1043.

Budas GR, Churchill EN, Disatnik MH, Sun L, and Mochly-Rosen D (2010) Mitochondrial import of PKCepsilon is mediated by HSP90: a role in cardioprotection from ischaemia and reperfusion injury. Cardiovasc Res 88:83-92.

Bukauskas FF and Verselis VK (2004) Gap junction channel gating. Biochim Biophys Acta 1662:42-60.

Bukauskas FF, Elfgang C, Willecke K, and Weingart R (1995) Biophysical properties of gap junction channels formed by mouse connexin40 in induced pairs of transfected human HeLa cells. Biophys J 68:2289-2298. 
Bulluck H, Yellon DM, and Hausenloy DJ (2016) Reducing myocardial infarct size: challenges and future opportunities. Heart 102:341-348.

Burghardt RC, Barhoumi R, Sewall TC, and Bowen JA (1995) Cyclic AMP induces rapid increases in gap junction permeability and changes in the cellular distribution of connexin43. J Membr Biol 148:243-253.

Burra S and Jiang JX (2009) Connexin 43 hemichannel opening associated with Prostaglandin $\mathrm{E}(2)$ release is adaptively regulated by mechanical stimulation. Commun Integr Biol 2:239-240.

Burt JM (1987) Block of intercellular communication: interaction of intracellular $\mathrm{H}^{+}$ and $\mathrm{Ca}^{2+}$. Am J Physiol 253:C607-C612.

Burt JM and Spray DC (1989) Volatile anesthetics block intercellular communication between neonatal rat myocardial cells. Circ Res 65:829-837.

Burt JM, Massey KD, and Minnich BN (1991) Uncoupling of cardiac cells by fatty acids: structure-activity relationships. Am J Physiol 260:C439-C448.

Butera JA, Larsen BD, Hennan JK, Kerns E, Di L, Alimardanov A, Swillo RE Morgan GA, Liu K, Wang Q, et al. (2009) Discovery of (2S,4R)-1-(2-aminoacetyl)-4benzamidopyrrolidine-2-carboxylic acid hydrochloride (GAP-134)13, an orally active small molecule gap-junction modifier for the treatment of atrial fibrillation. $J$ Med Chem 52:908-911.

Butterweck A, Gergs U, Elfgang C, Willecke K, and Traub O (1994) Immunochemical characterization of the gap junction protein connexin 45 in mouse kidney and transfected human HeLa cells. J Membr Biol 141:247-256.

Cabo C and Boyden PA (2009) Extracellular space attenuates the effect of gap junctional remodeling on wave propagation: a computational study. Biophys $J \mathbf{9 6}$ 3092-3101.

Calder BW, Matthew Rhett J, Bainbridge H, Fann SA, Gourdie RG, and Yost MJ (2015) Inhibition of connexin 43 hemichannel-mediated ATP release attenuates early inflammation during the foreign body response. Tissue Eng Part A 21 : $1752-1762$.

Calero G, Kanemitsu M, Taffet SM, Lau AF, and Delmar M (1998) A 17mer peptide interferes with acidification-induced uncoupling of connexin43. Circ Res 82 929-935.

Callis TE, Pandya K, Seok HY, Tang RH, Tatsuguchi M, Huang ZP, Chen JF, Deng Z Gunn B, Shumate J, et al. (2009) MicroRNA-208a is a regulator of cardiac hypertrophy and conduction in mice. J Clin Invest 119:2772-2786.

Camelliti P, Devlin GP, Matthews KG, Kohl P, and Green CR (2004) Spatially and temporally distinct expression of fibroblast connexins after sheep ventricular infarction. Cardiovasc Res 62:415-425.

Cameron SJ, Malik S, Akaike M, Lerner-Marmarosh N, Yan C, Lee JD, Abe J, and Yang J (2003) Regulation of epidermal growth factor-induced connexin 43 gap junction communication by big mitogen-activated protein kinase1/ERK5 but not ERK1/2 kinase activation. J Biol Chem 278:18682-18688.

Caruso RA, Fedele F, Finocchiaro G, Pizzi G, Nunnari M, Gitto G, Fabiano V, Parisi A, and Venuti A (2009) Ultrastructural descriptions of pericyte/endothelium pegsocket interdigitations in the microvasculature of human gastric carcinomas. Anticancer Res 29:449-453.

Castellano P and Eugenin EA (2014) Regulation of gap junction channels by infectious agents and inflammation in the CNS. Front Cell Neurosci 8:122.

Catarino S, Ramalho JS, Marques C, Pereira P, and Girão H (2011) Ubiquitinmediated internalization of connexin43 is independent of the canonical endocytic tyrosine-sorting signal. Biochem J 437:255-267.

Cea LA, Cisterna BA, Puebla C, Frank M, Figueroa XF, Cardozo C, Willecke K Latorre R, and Sáez JC (2013) De novo expression of connexin hemichannels in denervated fast skeletal muscles leads to atrophy. Proc Natl Acad Sci USA 110 16229-16234.

Cea LA, Balboa E, Puebla C, Vargas AA, Cisterna BA, Escamilla R, Regueira T, and Sáez JC (2016a) Dexamethasone-induced muscular atrophy is mediated by functional expression of connexin-based hemichannels. Biochim Biophys Acto 1862:1891-1899.

Cea LA, Bevilacqua JA, Arriagada C, Cárdenas AM, Bigot A, Mouly V, Sáez JC, and Caviedes P (2016b) The absence of dysferlin induces the expression of functional connexin-based hemichannels in human myotubes. BMC Cell Biol 17 (Suppl 1): 15

Cea LA, Puebla C, Cisterna BA, Escamilla R, Vargas AA, Frank M, MartínezMontero P, Prior C, Molano J, Esteban-Rodríguez I, et al. (2016c) Fast skeletal myofibers of mdx mouse, model of Duchenne muscular dystrophy, express connexin hemichannels that lead to apoptosis. Cell Mol Life Sci 73:2583-2599.

Cerrone M, Lin X, Zhang M, Agullo-Pascual E, Pfenniger A, Chkourko Gusky H, Novelli V, Kim C, Tirasawadichai T, Judge DP, et al. (2014) Missense mutations in plakophilin-2 cause sodium current deficit and associate with a Brugada syndrome phenotype. Circulation 129:1092-1103.

Chaabane C, Otsuka F, Virmani R, and Bochaton-Piallat ML (2013) Biological responses in stented arteries. Cardiovasc Res 99:353-363.

Chadjichristos CE, Matter CM, Roth I, Sutter E, Pelli G, Lüscher TF, Chanson M, and Kwak BR (2006) Reduced connexin43 expression limits neointima formation after balloon distension injury in hypercholesterolemic mice. Circulation 113 $2835-2843$

Chadjichristos CE, Scheckenbach KE, van Veen TA, Richani Sarieddine MZ, de Wit C, Yang Z, Roth I, Bacchetta M, Viswambharan H, Foglia B, et al. (2010) Endothelial-specific deletion of connexin 40 promotes atherosclerosis by increasing CD73-dependent leukocyte adhesion. Circulation 121:123-131.

Chakravarthy BR, Wang J, Tremblay R, Atkinson TG, Wang F, Li H, Buchan AM, and Durkin JP (1998) Comparison of the changes in protein kinase C induced by glutamate in primary cortical neurons and by in vivo cerebral ischaemia. Cell Signal 10:291-295.

Chan DK and Chang KW (2014) GJB2-associated hearing loss: systematic review of worldwide prevalence, genotype, and auditory phenotype. Laryngoscope 124: E34-E53.

Chandrasekhar A and Bera AK (2012) Hemichannels: permeants and their effect on development, physiology and death. Cell Biochem Funct 30:89-100.
Chang SJ, Tzeng CR, Lee YH, and Tai CJ (2008) Extracellular ATP activates the $\mathrm{PLC} / \mathrm{PKC} / \mathrm{ERK}$ signaling pathway through the $\mathrm{P} 2 \mathrm{Y} 2$ purinergic receptor leading to the induction of early growth response 1 expression and the inhibition of viability in human endometrial stromal cells. Cell Signal 20:1248-1255.

Chaytor AT, Evans WH, and Griffith TM (1997) Peptides homologous to extracellular loop motifs of connexin 43 reversibly abolish rhythmic contractile activity in rabbit arteries. J Physiol 503:99-110.

Chekeni FB, Elliott MR, Sandilos JK, Walk SF, Kinchen JM, Lazarowski ER, Armstrong AJ, Penuela S, Laird DW, Salvesen GS, et al. (2010) Pannexin 1 channels mediate 'find-me' signal release and membrane permeability during apoptosis. Nature 467:863-867.

Chen CC, Lien HY, Hsu YJ, Lin CC, Shih CM, and Lee TM (2010) Effect of pravastatin on ventricular arrhythmias in infarcted rats: role of connexin43. J Appl Physiol (1985) 109:541-552.

Chen G, Park CK, Xie RG, Berta T, Nedergaard M, and Ji RR (2014) Connexin-43 induces chemokine release from spinal cord astrocytes to maintain late-phase neuropathic pain in mice. Brain 137:2193-2209.

Chen G, Zhao J, Liu C, Zhang Y, Huo Y, and Zhou L (2015a) MG132 proteasome inhibitor upregulates the expression of connexin 43 in rats with adriamycininduced heart failure. Mol Med Rep 12:7595-7602.

Chen HJ, Yao L, Chen TG, Yu M, Wang LH, and Chen JZ (2007) Atorvastatin prevents connexin43 remodeling in hypertrophied left ventricular myocardium of spontaneously hypertensive rats. Chin Med J (Engl) 120:1902-1907.

Chen J, Pan L, Wei Z, Zhao Y, and Zhang M (2008) Domain-swapped dimerization of ZO-1 PDZ2 generates specific and regulatory connexin43-binding sites. EMBO J 27:2113-2123.

Chen M and Jones DL (2000) Age- and myopathy-dependent changes in connexins of normal and cardiomyopathic Syrian hamster ventricular myocardium. Can $J$ Physiol Pharmacol 78:669-678.

Chen MJ, Kress B, Han X, Moll K, Peng W, Ji RR, and Nedergaard M (2012a) Astrocytic CX43 hemichannels and gap junctions play a crucial role in development of chronic neuropathic pain following spinal cord injury. Glia 60:1660-1670.

Chen Q, Boire A, Jin X, Valiente M, Er EE, Lopez-Soto A, Jacob LS, Patwa R, Shah $\mathrm{H}, \mathrm{Xu} \mathrm{K}$, et al. (2016) Carcinoma-astrocyte gap junctions promote brain metastasis by cGAMP transfer. Nature 533:493-498.

Chen VC, Kristensen AR, Foster LJ, and Naus CC (2012b) Association of connexin43 with E3 ubiquitin ligase TRIM21 reveals a mechanism for gap junction phosphodegron control. J Proteome Res 11:6134-6146.

Chen VC, Gouw JW, Naus CC, and Foster LJ (2013) Connexin multi-site phosphorylation: mass spectrometry-based proteomics fills the gap. Biochim Biophys Acta 1828:23-34.

Chen Y and Swanson RA (2003) Astrocytes and brain injury. J Cereb Blood Flow Metab 23:137-149.

Chen YS, Green CR, Danesh-Meyer HV, and Rupenthal ID (2015b) Neuroprotection in the treatment of glaucoma-A focus on connexin 43 gap junction channel blockers. Eur J Pharm Biopharm 95 (Pt B):182-193.

Chen YS, Green CR, Teague R, Perrett J, Danesh-Meyer HV, Toth I, and Rupentha ID (2015c) Intravitreal injection of lipoamino acid-modified connexin43 mimetic peptide enhances neuroprotection after retinal ischemia. Drug Deliv Transl Res 5: 480-488.

Chen YS, Green CR, Wang K, Danesh-Meyer HV, and Rupenthal ID (2015d) Sustained intravitreal delivery of connexin 43 mimetic peptide by poly(D,L-lactide-coglycolide) acid micro- and nanoparticles-closing the gap in retinal ischaemia. Eur $J$ Pharm Biopharm 95 (Pt B):378-386.

Cherian PP, Siller-Jackson AJ, Gu S, Wang X, Bonewald LF, Sprague E, and Jiang JX (2005) Mechanical strain opens connexin 43 hemichannels in osteocytes: a novel mechanism for the release of prostaglandin. Mol Biol Cell 16:3100-3106.

Chi W, Chen H, Li F, Zhu Y, Yin W, and Zhuo Y (2015) HMGB1 promotes the activation of NLRP3 and caspase-8 inflammasomes via NF-kB pathway in acute glaucoma. J Neuroinflammation 12:137.

Chowdhury D, Marco S, Brooks IM, Zandueta A, Rao Y, Haucke V, Wesseling JF, Tavalin SJ, and Pérez-Otaño I (2013) Tyrosine phosphorylation regulates the endocytosis and surface expression of GluN3A-containing NMDA receptors. $J$ Neurosci 33:4151-4164

Chu FF and Doyle D (1985) Turnover of plasma membrane proteins in rat hepatoma cells and primary cultures of rat hepatocytes. J Biol Chem 260:3097-3107.

Chung TH, Wang SM, and Wu JC (2004) 17beta-estradiol reduces the effect of metabolic inhibition on gap junction intercellular communication in rat cardiomyocytes via the estrogen receptor. J Mol Cell Cardiol 37:1013-1022.

Chung TH, Wang SM, Chang YC, Chen YL, and Wu JC (2007) 18beta-glycyrrhetinic acid promotes src interaction with connexin 43 in rat cardiomyocytes. J Cell Biochem 100:653-664.

Churchill GC, Lurtz MM, and Louis CF (2001) $\mathrm{Ca}^{(2+)}$ regulation of gap junctional coupling in lens epithelial cells. Am J Physiol Cell Physiol 281:C972-C981.

Churko JM, Langlois S, Pan X, Shao Q, and Laird DW (2010) The potency of the fs260 connexin43 mutant to impair keratinocyte differentiation is distinct from other disease-linked connexin43 mutants. Biochem J 429:473-483.

Churko JM, Shao Q, Gong XQ, Swoboda KJ, Bai D, Sampson J, and Laird DW (2011) Human dermal fibroblasts derived from oculodentodigital dysplasia patients suggest that patients may have wound-healing defects. Hum Mutat 32: $456-466$

Cina C, Bechberger JF, Ozog MA, and Naus CC (2007) Expression of connexins in embryonic mouse neocortical development. J Comp Neurol 504:298-313.

Clarke TC, Thomas D, Petersen JS, Evans WH, and Martin PE (2006) The antiarrhythmic peptide rotigaptide (ZP123) increases gap junction intercellular communication in cardiac myocytes and HeLa cells expressing connexin $43 . \mathrm{Br} J$ Pharmacol 147:486-495.

Clarke TC, Williams OJ, Martin PE, and Evans WH (2009) ATP release by cardiac myocytes in a simulated ischaemia model: inhibition by a connexin mimetic and enhancement by an antiarrhythmic peptide. Eur J Pharmacol 605:9-14. 
Cochrane S, Bergoffen J, Fairweather ND, Müller E, Mostacciuolo ML, Monaco AP, Fischbeck KH, and Haites NE (1994) X linked Charcot-Marie-Tooth disease (CMTX1): a study of 15 families with 12 highly informative polymorphisms. J Med Genet 31:193-196.

Colussi C, Berni R, Rosati J, Straino S, Vitale S, Spallotta F, Baruffi S, Bocchi L, Delucchi F, Rossi S, et al. (2010) The histone deacetylase inhibitor suberoylanilide hydroxamic acid reduces cardiac arrhythmias in dystrophic mice. Cardiovasc Res 87:73-82.

Colussi C, Rosati J, Straino S, Spallotta F, Berni R, Stilli D, Rossi S, Musso E, Macchi E, Mai A, et al. (2011) Ne-lysine acetylation determines dissociation from GAP junctions and lateralization of connexin 43 in normal and dystrophic heart. Proc Natl Acad Sci USA 108:2795-2800.

Condorelli DF, Parenti R, Spinella F, Trovato Salinaro A, Belluardo N, Cardile V, and Cicirata F (1998) Cloning of a new gap junction gene (Cx36) highly expressed in mammalian brain neurons. Eur J Neurosci 10:1202-1208.

Connors BW (2012) Tales of a dirty drug: carbenoxolone, gap junctions, and seizures. Epilepsy Curr 12:66-68.

Contreras JE, Sánchez HA, Eugenin EA, Speidel D, Theis M, Willecke K, Bukauskas FF, Bennett MV, and Sáez JC (2002) Metabolic inhibition induces opening of unapposed connexin 43 gap junction hemichannels and reduces gap junctional communication in cortical astrocytes in culture. Proc Natl Acad Sci USA 99: 495-500.

Contreras JE, Sáez JC, Bukauskas FF, and Bennett MV (2003) Gating and regulation of connexin $43(\mathrm{Cx} 43)$ hemichannels. Proc Natl Acad Sci USA 100 11388-11393.

Contreras JE, Sánchez HA, Véliz LP, Bukauskas FF, Bennett MV, and Sáez JC (2004) Role of connexin-based gap junction channels and hemichannels in ischemia-induced cell death in nervous tissue. Brain Res Brain Res Rev 47: 290-303.

Cook DJ and Tymianski M (2011) Translating promising preclinical neuroprotective therapies to human stroke trials. Expert Rev Cardiovasc Ther 9: 433-449

Cook DJ, Teves L, and Tymianski M (2012) Treatment of stroke with a PSD-95 inhibitor in the gyrencephalic primate brain. Nature 483:213-217.

Cooper CD and Lampe PD (2002) Casein kinase 1 regulates connexin-43 gap junction assembly. J Biol Chem 277:44962-44968.

Coppen SR, Severs NJ, and Gourdie RG (1999) Connexin45 (alpha 6) expression delineates an extended conduction system in the embryonic and mature rodent heart. Dev Genet 24:82-90.

Coronel R, Fiolet JW, Wilms-Schopman JG, Opthof T, Schaapherder AF, and Janse MJ (1989) Distribution of extracellular potassium and electrophysiologic changes during two-stage coronary ligation in the isolated, perfused canine heart. Circulation 80:165-177.

Cotrina ML, Kang J, Lin JH, Bueno E, Hansen TW, He L, Liu Y, and Nedergaard M (1998) Astrocytic gap junctions remain open during ischemic conditions. $J$ Neurosci 18:2520-2537.

Cottrell GT, Lin R, Warn-Cramer BJ, Lau AF, and Burt JM (2003) Mechanism of $\mathrm{v}$-Src- and mitogen-activated protein kinase-induced reduction of gap junction communication. Am J Physiol Cell Physiol 284:C511-C520.

Cronin M, Anderson PN, Cook JE, Green CR, and Becker DL (2008) Blocking connexin43 expression reduces inflammation and improves functional recovery after spinal cord injury. Mol Cell Neurosci 39:152-160.

Crow DS, Beyer EC, Paul DL, Kobe SS, and Lau AF (1990) Phosphorylation of connexin43 gap junction protein in uninfected and Rous sarcoma virustransformed mammalian fibroblasts. Mol Cell Biol 10:1754-1763.

Crow JM, Atkinson MM, and Johnson RG (1994) Micromolar levels of intracellular calcium reduce gap junctional permeability in lens cultures. Invest Ophthalmol Vis Sci 35:3332-3341.

Cruciani V and Mikalsen SO (2006) The vertebrate connexin family. Cell Mol Life Sci 63:1125-1140

Cruikshank SJ, Hopperstad M, Younger M, Connors BW, Spray DC, and Srinivas M (2004) Potent block of Cx36 and Cx50 gap junction channels by mefloquine. Proc Natl Acad Sci USA 101:12364-12369.

Curcio A, Torella D, Iaconetti C, Pasceri E, Sabatino J, Sorrentino S, Giampà S, Micieli M, Polimeni A, Henning BJ, et al. (2013) MicroRNA-1 downregulation increases connexin 43 displacement and induces ventricular tachyarrhythmias in rodent hypertrophic hearts. PLoS One 8:e70158.

D'hondt C, Iyyathurai J, Wang N, Gourdie RG, Himpens B, Leybaert L, and Bultynck G (2013) Negatively charged residues (Asp378 and Asp379) in the last ten amino acids of the C-terminal tail of $\mathrm{Cx} 43$ hemichannels are essential for loop/tail interactions. Biochem Biophys Res Commun 432:707-712.

Dahl G (2007) Gap junction-mimetic peptides do work, but in unexpected ways. Cell Commun Adhes 14:259-264.

Dahl G, Qiu F, and Wang J (2013) The bizarre pharmacology of the ATP release channel pannexin1. Neuropharmacology 75:583-593.

Dakin K, Zhao Y, and Li WH (2005) LAMP, a new imaging assay of gap junctional communication unveils that $\mathrm{Ca}^{2+}$ influx inhibits cell coupling. Nat Methods 2 55-62.

Danesh-Meyer HV, Huang R, Nicholson LF, and Green CR (2008) Connexin43 antisense oligodeoxynucleotide treatment down-regulates the inflammatory response in an in vitro interphase organotypic culture model of optic nerve ischaemia. J Clin Neurosci 15:1253-1263.

Danesh-Meyer HV, Kerr NM, Zhang J, Eady EK, O'Carroll SJ, Nicholson LF, Johnson CS, and Green CR (2012) Connexin43 mimetic peptide reduces vascular leak and retinal ganglion cell death following retinal ischaemia. Brain 135: 506-520.

Danesh-Meyer HV, Zhang J, Acosta ML, Rupenthal ID, and Green CR (2016) Connexin43 in retinal injury and disease. Prog Retin Eye Res 51:41-68.

Dang X, Doble BW, and Kardami E (2003) The carboxy-tail of connexin-43 localizes to the nucleus and inhibits cell growth. Mol Cell Biochem 242:35-38.
Danielson LS, Park DS, Rotllan N, Chamorro-Jorganes A, Guijarro MV, FernandezHernando C, Fishman GI, Phoon CK, and Hernando E (2013) Cardiovascular dysregulation of miR-17-92 causes a lethal hypertrophic cardiomyopathy and arrhythmogenesis. FASEB J 27:1460-1467.

Danik SB, Rosner G, Lader J, Gutstein DE, Fishman GI, and Morley GE (2008) Electrical remodeling contributes to complex tachyarrhythmias in connexin43deficient mouse hearts. FASEB J 22:1204-1212.

Darrow BJ, Laing JG, Lampe PD, Saffitz JE, and Beyer EC (1995) Expression of multiple connexins in cultured neonatal rat ventricular myocytes. Circ Res $\mathbf{7 6}$ 381-387.

Davidson JO, Green CR, Nicholson LF, Bennet L, and Gunn AJ (2012a) Deleterious effects of high dose connexin 43 mimetic peptide infusion after cerebral ischaemia in near-term fetal sheep. Int $J$ Mol Sci 13:6303-6319.

Davidson JO, Green CR, Nicholson LF, O'Carroll SJ, Fraser M, Bennet L, and Gunn AJ (2012b) Connexin hemichannel blockade improves outcomes in a model of fetal ischemia. Ann Neurol 71:121-132.

Davidson JO, Drury PP, Green CR, Nicholson LF, Bennet L, and Gunn AJ (2014) Connexin hemichannel blockade is neuroprotective after asphyxia in preterm fetal sheep. PLoS One 9:e96558.

Davidson JO, Rout AL, Wassink G, Yuill CA, Zhang FG, Green CR, Bennet L, and Gunn AJ (2015) Non-additive effects of delayed connexin hemichannel blockade and hypothermia after cerebral ischemia in near-term fetal sheep. $J$ Cereb Blood Flow Metab 35:2052-2061.

Davis LM, Kanter HL, Beyer EC, and Saffitz JE (1994) Distinct gap junction protein phenotypes in cardiac tissues with disparate conduction properties. J Am Coll Cardiol 24:1124-1132.

Davis LM, Rodefeld ME, Green K, Beyer EC, and Saffitz JE (1995) Gap junction protein phenotypes of the human heart and conduction system. $J$ Cardiovasc Electrophysiol 6:813-822.

Dbouk HA, Mroue RM, El-Sabban ME, and Talhouk RS (2009) Connexins: a myriad of functions extending beyond assembly of gap junction channels. Cell Commun Signal 7:4.

De Bock M, Culot M, Wang N, Bol M, Decrock E, De Vuyst E, da Costa A, Dauwe I, Vinken M, Simon AM, et al. (2011) Connexin channels provide a target to manipulate brain endothelial calcium dynamics and blood-brain barrier permeability. $J$ Cereb Blood Flow Metab 31:1942-1957.

De Bock M, Wang N, Bol M, Decrock E, Ponsaerts R, Bultynck G, Dupont G, and Leybaert L (2012) Connexin 43 hemichannels contribute to cytoplasmic $\mathrm{Ca}^{2+}$ oscillations by providing a bimodal $\mathrm{Ca}^{2+}$-dependent $\mathrm{Ca}^{2+}$ entry pathway. $J$ Biol Chem 287:12250-12266.

De Bock M, Kerrebrouck M, Wang N, and Leybaert L (2013a) Neurological manifestations of oculodentodigital dysplasia: a $\mathrm{Cx} 43$ channelopathy of the central nervous system? Front Pharmacol 4:120.

De Bock M, Wang N, Decrock E, Bol M, Gadicherla AK, Culot M, Cecchelli R, Bultynck G, and Leybaert L (2013b) Endothelial calcium dynamics, connexin channels and blood-brain barrier function. Prog Neurobiol 108:1-20.

De Bock M, Decrock E, Wang N, Bol M, Vinken M, Bultynck G, and Leybaert L (2014a) The dual face of connexin-based astroglial $\mathrm{Ca}^{(2+)}$ communication: a key player in brain physiology and a prime target in pathology. Biochim Biophys Acta 1843:2211-2232.

De Bock M, Vandenbroucke RE, Decrock E, Culot M, Cecchelli R, and Leybaert L (2014b) A new angle on blood-CNS interfaces: a role for connexins? FEBS Lett 588: $1259-1270$.

de Groot JR, Wilms-Schopman FJ, Opthof T, Remme CA, and Coronel R (2001) Late ventricular arrhythmias during acute regional ischemia in the isolated blood perfused pig heart. Role of electrical cellular coupling. Cardiovasc Res 50:362-372.

De Mello W and Altieri P (1992) The role of the renin-angiotensin system in the control of cell communication in the heart: effects of enalapril and angiotensin II. $J$ Cardiovasc Pharmacol 20:643-651.

De Mello WC (1984) Effect of intracellular injection of cAMP on the electrical coupling of mammalian cardiac cells. Biochem Biophys Res Commun 119:1001-1007.

De Mello WC (1997) Influence of alpha-adrenergic-receptor activation on junctional conductance in heart cells: interaction with beta-adrenergic adrenergic agonists. $J$ Cardiovasc Pharmacol 29:273-277.

De Mello WC and Thormahlen D (1999) Effect of tedisamil on cell communication, impulse propagation, and excitability of the failing heart. Eur $J$ Pharmacol 372: 241-246.

De Pina-Benabou MH, Srinivas M, Spray DC, and Scemes E (2001) Calmodulin kinase pathway mediates the $\mathrm{K}^{+}$-induced increase in Gap junctional communication between mouse spinal cord astrocytes. J Neurosci 21:6635-6643.

De Vuyst E, Decrock E, Cabooter L, Dubyak GR, Naus CC, Evans WH, and Leybaert $\mathrm{L}$ (2006) Intracellular calcium changes trigger connexin 32 hemichannel opening. EMBO J 25:34-44

De Vuyst E, Decrock E, De Bock M, Yamasaki H, Naus CC, Evans WH, and Leybaert L (2007) Connexin hemichannels and gap junction channels are differentially influenced by lipopolysaccharide and basic fibroblast growth factor. Mol Biol Cell 18:34-46.

De Vuyst E, Wang N, Decrock E, De Bock M, Vinken M, Van Moorhem M, Lai C, Culot M, Rogiers V, Cecchelli $\mathrm{R}$, et al. (2009) $\mathrm{Ca}^{(2+)}$ regulation of connexin 43 hemichannels in C6 glioma and glial cells. Cell Calcium 46:176-187.

de Wit C and Griffith TM (2010) Connexins and gap junctions in the EDHF phenomenon and conducted vasomotor responses. Pflugers Arch 459:897-914.

De Zeeuw CI, Chorev E, Devor A, Manor Y, Van Der Giessen RS, De Jeu MT, Hoogenraad CC, Bijman J, Ruigrok TJ, French P, et al. (2003) Deformation of network connectivity in the inferior olive of connexin 36-deficient mice is compensated by morphological and electrophysiological changes at the single neuron level. $J \mathrm{Neu}$ rosci 23:4700-4711.

Deans MR, Gibson JR, Sellitto C, Connors BW, and Paul DL (2001) Synchronous activity of inhibitory networks in neocortex requires electrical synapses containing connexin36. Neuron 31:477-485. 
Decrock E, De Vuyst E, Vinken M, Van Moorhem M, Vranckx K, Wang N, Van Laeken L, De Bock M, D'Herde K, Lai CP, et al. (2009a) Connexin 43 hemichannels contribute to the propagation of apoptotic cell death in a rat $\mathrm{C} 6$ glioma cell model. Cell Death Differ 16:151-163.

Decrock E, Vinken M, De Vuyst E, Krysko DV, D’Herde K, Vanhaecke T, Vandenabeele P, Rogiers V, and Leybaert L (2009b) Connexin-related signaling in cell death: to live or let die? Cell Death Differ 16:524-536.

Decrock E, De Bock M, Wang N, Bultynck G, Giaume C, Naus CC, Green CR, and Leybaert L (2015) Connexin and pannexin signaling pathways, an architectural blueprint for CNS physiology and pathology? Cell Mol Life Sci 72:2823-2851.

Decrock E, Hoorelbeke D, Ramadan R, Delvaeye T, De Bock M, Wang N, Krysko DV Baatout S, Bultynck G, Aerts A, et al. (2017) Calcium, oxidative stress and connexin channels, a harmonious orchestra directing the response to radiotherapy treatment? Biochim Biophys Acta 1864:1099-1120.

Dekker LR, Fiolet JW, VanBavel E, Coronel R, Opthof T, Spaan JA, and Janse MJ (1996) Intracellular $\mathrm{Ca}^{2+}$, intercellular electrical coupling, and mechanical activity in ischemic rabbit papillary muscle. Effects of preconditioning and metabolic blockade. Circ Res 79:237-246.

Delage B and Délèze J (1998) Increase of the gap junction conductance of adult mammalian heart myocytes by intracellular calcium ions, in Gap Junctions (Werner R, ed) IOS Press, Amsterdam, The Netherlands.

Delmar M and Makita N (2012) Cardiac connexins, mutations and arrhythmias. Curr Opin Cardiol 27:236-241.

Delmar M, Michaels DC, Johnson T, and Jalife J (1987) Effects of increasing intercellular resistance on transverse and longitudinal propagation in sheep epicardial muscle. Circ Res 60:780-785.

Denuc A, Núñez E, Calvo E, Loureiro M, Miro-Casas E, Guarás A, Vázquez J, and Garcia-Dorado D (2016) New protein-protein interactions of mitochondrial connexin 43 in mouse heart. J Cell Mol Med 20:794-803.

DePaola N, Davies PF, Pritchard WF Jr, Florez L, Harbeck N, and Polacek DC (1999) Spatial and temporal regulation of gap junction connexin43 in vascular endothelial cells exposed to controlled disturbed flows in vitro. Proc Natl Acad Sci USA 96: $3154-3159$

Dere E, Zheng-Fischhöfer Q, Viggiano D, Gironi Carnevale UA, Ruocco LA, Zlomuzica A, Schnichels M, Willecke K, Huston JP, and Sadile AG (2008) Connexin31.1 deficiency in the mouse impairs object memory and modulates open-field exploration, acetylcholine esterase levels in the striatum, and cAMP response element-binding protein levels in the striatum and piriform cortex. Neuroscience 153:396-405.

Dermietzel R, Meier C, Bukauskas F, and Spray DC (2003) Following tracks of hemichannels. Cell Commun Adhes 10:335-340.

Derouette JP, Desplantez T, Wong CW, Roth I, Kwak BR, and Weingart R (2009) Functional differences between human Cx37 polymorphic hemichannels. $J$ Mol Cell Cardiol 46:499-507.

Desplantez T, McCain ML, Beauchamp P, Rigoli G, Rothen-Rutishauser B, Parker $\mathrm{KK}$, and Kleber AG (2012a) Connexin43 ablation in foetal atrial myocytes decreases electrical coupling, partner connexins, and sodium current. Cardiovasc Res 94:58-65.

Desplantez T, Verma V, Leybaert L, Evans WH, and Weingart R (2012b) Gap26, a connexin mimetic peptide, inhibits currents carried by connexin 43 hemichannels and gap junction channels. Pharmacol Res 65:546-552.

Dhein S (2004) Pharmacology of gap junctions in the cardiovascular system. Cardiovasc Res 62:287-298.

Dhein S (2006a) Cardiac ischemia and uncoupling: gap junctions in ischemia and infarction. Adv Cardiol 42:198-212.

Dhein S (2006b) Role of connexins in atrial fibrillation. Adv Cardiol 42:161-174

Dhein S and Hammerath SB (2001) Aspects of the intercellular communication in aged hearts: effects of the gap junction uncoupler palmitoleic acid. Naunyn Schmiedebergs Arch Pharmacol 364:397-408.

Dhein S, Manicone N, Müller A, Gerwin R, Ziskoven U, Irankhahi A, Minke C, and Klaus W (1994) A new synthetic antiarrhythmic peptide reduces dispersion of epicardial activation recovery interval and diminishes alterations of epicardial activation patterns induced by regional ischemia. A mapping study. Naunyn Schmiedebergs Arch Pharmacol 350:174-184

Dhein S, Krüsemann K, and Schaefer T (1999) Effects of the gap junction uncoupler palmitoleic acid on the activation and repolarization wavefronts in isolated rabbit hearts. Br J Pharmacol 128:1375-1384.

Dhein S, Weng S, Grover R, Tudyka T, Gottwald M, Schaefer T, and Polontchouk L (2001) Protein kinase Calpha mediates the effect of antiarrhythmic peptide on gap junction conductance. Cell Commun Adhes 8:257-264.

Dhein S, Larsen BD, Petersen JS, and Mohr FW (2003) Effects of the new antiarrhythmic peptide ZP123 on epicardial activation and repolarization pattern. Cell Commun Adhes 10:371-378.

Dhein S, Hagen A, Jozwiak J, Dietze A, Garbade J, Barten M, Kostelka M, and Mohr FW (2010) Improving cardiac gap junction communication as a new antiarrhythmic mechanism: the action of antiarrhythmic peptides. Naunyn Schmiedebergs Arch Pharmacol 381:221-234.

Dhein S, Rothe S, Busch A, Rojas Gomez DM, Boldt A, Reutemann A, Seidel T, Salameh A, Pfannmüller B, Rastan A, et al. (2011) Effects of metoprolol therapy on cardiac gap junction remodelling and conduction in human chronic atrial fibrillation. Br J Pharmacol 164:607-616.

Dhein S, Seidel T, Salameh A, Jozwiak J, Hagen A, Kostelka M, Hindricks G, and Mohr FW (2014) Remodeling of cardiac passive electrical properties and susceptibility to ventricular and atrial arrhythmias. Front Physiol 5:424

Diestel S, Eckert R, Hülser D, and Traub O (2004) Exchange of serine residues 263 and 266 reduces the function of mouse gap junction protein connexin 31 and exhibits a dominant-negative effect on the wild-type protein in HeLa cells. Exp Cell Res 294:446-457.

Díez JA, Elvira M, and Villalobo A (1998) The epidermal growth factor receptor tyrosine kinase phosphorylates connexin32. Mol Cell Biochem 187:201-210.
Doble BW and Kardami E (1995) Basic fibroblast growth factor stimulates connexin43 expression and intercellular communication of cardiac fibroblasts. Mol Cell Biochem 143:81-87.

Doble BW, Ping P, and Kardami E (2000) The epsilon subtype of protein kinase $\mathrm{C}$ is required for cardiomyocyte connexin- 43 phosphorylation. Circ Res 86: 293-301

Doble BW, Dang X, Ping P, Fandrich RR, Nickel BE, Jin Y, Cattini PA, and Kardami E (2004) Phosphorylation of serine 262 in the gap junction protein connexin-43 regulates DNA synthesis in cell-cell contact forming cardiomyocytes. J Cell Sci 117:507-514.

Dobrenis K, Chang HY, Pina-Benabou MH, Woodroffe A, Lee SC, Rozental R, Spray DC, and Scemes E (2005) Human and mouse microglia express connexin36, and functional gap junctions are formed between rodent microglia and neurons. $J$ Neurosci Res 82:306-315.

Dobrowolski R, Sommershof A and Willecke K (2007) Some oculodentodigital dysplasia-associated $\mathrm{Cx} 43$ mutations cause increased hemichannel activity in addition to deficient gap junction channels. J Membr Biol 219:9-17.

Dobrowolski R, Sasse P, Schrickel JW, Watkins M, Kim JS, Rackauskas M, Troatz C, Ghanem A, Tiemann K, Degen J, et al. (2008) The conditional connexin43G138R mouse mutant represents a new model of hereditary oculodentodigital dysplasia in humans. Hum Mol Genet 17:539-554.

Dobrowolski R, Hertig G, Lechner H, Wörsdörfer P, Wulf V, Dicke N, Eckert D, Bauer $\mathrm{R}$, Schorle H, and Willecke K (2009) Loss of connexin43-mediated gap junctional coupling in the mesenchyme of limb buds leads to altered expression of morphogens in mice. Hum Mol Genet 18:2899-2911.

Dong F, Yang XJ, Jiang TB, and Chen Y (2016) Ischemia triggered ATP release through Pannexin-1 channel by myocardial cells activates sympathetic fibers. Microvasc Res 104:32-37.

dos Santos DO, Blefari V, Prado FP, Silva CA, Fazan R Jr, Salgado HC, Ramos SG, and Prado CM (2016) Reduced expression of adherens and gap junction proteins can have a fundamental role in the development of heart failure following cardiac hypertrophy in rats. Exp Mol Pathol 100:167-176.

Duchêne A, Perier M, Zhao Y, Liu X, Thomasson J, Chauveau F, Piérard C, Lagarde D, Picoli C, Jeanson T, et al. (2016) Impact of astroglial connexins on modafinil pharmacological properties. Sleep 39:1283-1292.

Duerrschmidt N, Hagen A, Gaertner C, Wermke A, Nowicki M, Spanel-Borowski K, Stepan H, Mohr FW, and Dhein S (2012) Nicotine effects on human endothelial intercellular communication via $\alpha 4 \beta 2$ and $\alpha 3 \beta 2$ nicotinic acetylcholine receptor subtypes. Naunyn Schmiedebergs Arch Pharmacol 385:621-632.

Duffy HS and Wit AL (2008) Is there a role for remodeled connexins in AF? No simple answers. J Mol Cell Cardiol 44:4-13.

Duffy HS, Sorgen PL, Girvin ME, O’Donnell P, Coombs W, Taffet SM, Delmar M, and Spray DC (2002) pH-dependent intramolecular binding and structure involving Cx43 cytoplasmic domains. J Biol Chem 277:36706-36714.

Duffy HS, Ashton AW, O'Donnell P, Coombs W, Taffet SM, Delmar M, and Spray DC (2004) Regulation of connexin43 protein complexes by intracellular acidification. Circ Res 94:215-222.

Dunham B, Liu S, Taffet S, Trabka-Janik E, Delmar M, Petryshyn R, Zheng S, Perzova R, and Vallano ML (1992) Immunolocalization and expression of functional and nonfunctional cell-to-cell channels from wild-type and mutant rat heart connexin43 cDNA. Circ Res 70:1233-1243.

Dunn CA and Lampe PD (2014) Injury-triggered Akt phosphorylation of Cx43: a ZO-1-driven molecular switch that regulates gap junction size. J Cell Sci 127: $455-464$.

Dunn CA, Su V, Lau AF, and Lampe PD (2012) Activation of Akt, not connexin 43 protein ubiquitination, regulates gap junction stability. $J$ Biol Chem 287: $2600-2607$.

Dupont E, Matsushita T, Kaba RA, Vozzi C, Coppen SR, Khan N, Kaprielian R Yacoub MH, and Severs NJ (2001) Altered connexin expression in human congestive heart failure. J Mol Cell Cardiol 33:359-371.

Easton JA, Petersen JS, and Martin PE (2009) The anti-arrhythmic peptide AAP10 remodels Cx43 and Cx40 expression and function. Naunyn Schmiedebergs Arch Pharmacol 380:11-24.

Ebihara L and Steiner E (1993) Properties of a nonjunctional current expressed from a rat connexin 46 cDNA in Xenopus oocytes. J Gen Physiol 102:59-74.

Ebihara L, Liu X, and Pal JD (2003) Effect of external magnesium and calcium on human connexin46 hemichannels. Biophys $J$ 84:277-286.

Eckert R (2006) Gap-junctional single-channel permeability for fluorescent tracers in mammalian cell cultures. Biophys $J$ 91:565-579.

Eidelman E, Chosack A, and Wagner ML (1967) Orodigitofacial dysostosis and oculodentodigital dysplasia. Two distinct syndromes with some similarities. Oral Surg Oral Med Oral Pathol 23:311-319.

Ek JF, Delmar M, Perzova R, and Taffet SM (1994) Role of histidine 95 on pH gating of the cardiac gap junction protein connexin43. Circ Res 74:1058-1064.

Ek-Vitorin JF and Burt JM (2013) Structural basis for the selective permeability of channels made of communicating junction proteins. Biochim Biophys Acta 1828: $51-68$.

Ek-Vitorin JF, King TJ, Heyman NS, Lampe PD, and Burt JM (2006) Selectivity of connexin 43 channels is regulated through protein kinase C-dependent phosphorylation. Circ Res 98:1498-1505.

el-Sherif N, Caref EB, Yin H, and Restivo M (1996) The electrophysiological mechanism of ventricular arrhythmias in the long QT syndrome. Tridimensional mapping of activation and recovery patterns. Circ Res 79:474-492.

Elias LA and Kriegstein AR (2008) Gap junctions: multifaceted regulators of embryonic cortical development. Trends Neurosci 31:243-250.

Elias LA, Wang DD, and Kriegstein AR (2007) Gap junction adhesion is necessary for radial migration in the neocortex. Nature 448:901-907.

Elias LA, Turmaine M, Parnavelas JG, and Kriegstein AR (2010) Connexin 43 mediates the tangential to radial migratory switch in ventrally derived cortical interneurons. J Neurosci 30:7072-7077. 
Eltzschig HK, Eckle T, Mager A, Küper N, Karcher C, Weissmüller T, Boengler K, Schulz R, Robson SC, and Colgan SP (2006) ATP release from activated neutrophils occurs via connexin 43 and modulates adenosine-dependent endothelial cell function. Circ Res 99:1100-1108.

Emdad L, Uzzaman M, Takagishi Y, Honjo H, Uchida T, Severs NJ, Kodama I, and Murata Y (2001) Gap junction remodeling in hypertrophied left ventricles of aortic-banded rats: prevention by angiotensin II type 1 receptor blockade. $\mathrm{J} \mathrm{Mol}$ Cell Cardiol 33:219-231.

Erickson HP (2009) Size and shape of protein molecules at the nanometer level determined by sedimentation, gel filtration, and electron microscopy. Biol Proced Online 11:32-51.

Esseltine JL and Laird DW (2016) Next-generation connexin and pannexin cell biology. Trends Cell Biol 26:944-955.

Esseltine JL, Shao Q, Huang T, Kelly JJ, Sampson J, and Laird DW (2015) Manipulating $\mathrm{Cx} 43$ expression triggers gene reprogramming events in dermal fibroblasts from oculodentodigital dysplasia patients. Biochem J 472:55-69.

Esseltine JL, Shao Q, Brooks C, Sampson J, Betts DH, Seguin CA, and Laird DW (2017) Connexin43 mutant patient-derived induced pluripotent stem cells exhibit altered differentiation potential. J Bone Miner Res 32:1368-1385.

Eugenín EA, Eckardt D, Theis M, Willecke K, Bennett MV, and Saez JC (2001) Microglia at brain stab wounds express connexin 43 and in vitro form functional gap junctions after treatment with interferon-gamma and tumor necrosis factoralpha. Proc Natl Acad Sci USA 98:4190-4195.

Evans WH (2015) Cell communication across gap junctions: a historical perspective and current developments. Biochem Soc Trans 43:450-459.

Evans WH and Boitano S (2001) Connexin mimetic peptides: specific inhibitors of gap-junctional intercellular communication. Biochem Soc Trans 29:606-612.

Ezan P, André P, Cisternino S, Saubaméa B, Boulay AC, Doutremer S, Thomas MA, Quenech'du N, Giaume C, and Cohen-Salmon M (2012) Deletion of astroglial connexins weakens the blood-brain barrier. J Cereb Blood Flow Metab 32: 1457-1467.

Falk MM, Baker SM, Gumpert AM, Segretain D, and Buckheit RW III (2009) Gap junction turnover is achieved by the internalization of small endocytic doublemembrane vesicles. Mol Biol Cell 20:3342-3352.

Falk MM, Fong JT, Kells RM, O’Laughlin MC, Kowal TJ, and Thévenin AF (2012) Degradation of endocytosed gap junctions by autophagosomal and endo-/lysosomal pathways: a perspective. J Membr Biol 245:465-476.

Fang JS, Angelov SN, Simon AM, and Burt JM (2012) Cx40 is required for, and cx37 limits, postischemic hindlimb perfusion, survival and recovery. $J$ Vasc Res 49:2-12.

Fang JS, Angelov SN, Simon AM, and Burt JM (2013) Compromised regulation of tissue perfusion and arteriogenesis limit, in an AT1R-independent fashion, recovery of ischemic tissue in Cx40(-/-) mice. Am J Physiol Heart Circ Physiol 304 H816-H827.

Fannin J, Rice KM, Thulluri S, Dornon L, Arvapalli RK, Wehner P, and Blough ER (2014) Age-associated alterations of cardiac structure and function in the female F344xBN rat heart. Age (Dordr) 36:9684.

Fasciani I, Temperán A, Pérez-Atencio LF, Escudero A, Martínez-Montero P, Molano J, Gómez-Hernández JM, Paino CL, González-Nieto D, and Barrio LC (2013) Regulation of connexin hemichannel activity by membrane potential and the extracellular calcium in health and disease. Neuropharmacology 75:479-490.

Farkas E and Luiten PG (2001) Cerebral microvascular pathology in aging and Alzheimer's disease. Prog Neurobiol 64:575-611.

Feaver RE, Hastings NE, Pryor A, and Blackman BR (2008) GRP78 upregulation by atheroprone shear stress via p38-, alpha2beta1-dependent mechanism in endothelial cells. Arterioscler Thromb Vasc Biol 28:1534-1541.

Feigin VL, Forouzanfar MH, Krishnamurthi R, Mensah GA, Connor M, Bennett DA Moran AE, Sacco RL, Anderson L, Truelsen T, et al.; Global Burden of Diseases, Injuries, and Risk Factors Study 2010 (GBD 2010) and the GBD Stroke Experts Group (2014) Global and regional burden of stroke during 1990-2010: findings from the Global Burden of Disease Study 2010. Lancet 383:245-254.

Ferdinandy P, Hausenloy DJ, Heusch G, Baxter GF, and Schulz R (2014) Interaction of risk factors, comorbidities, and comedications with ischemia/reperfusion injury and cardioprotection by preconditioning, postconditioning, and remote conditioning. Pharmacol Rev 66:1142-1174.

Figueroa XF, Lillo MA, Gaete PS, Riquelme MA, and Sáez JC (2013) Diffusion of nitric oxide across cell membranes of the vascular wall requires specific connexinbased channels. Neuropharmacology 75:471-478.

Fiori MC, Figueroa V, Zoghbi ME, Saéz JC, Reuss L, and Altenberg GA (2012) Permeation of calcium through purified connexin 26 hemichannels. J Biol Chem 287:40826-40834.

Fiorini C, Gilleron J, Carette D, Valette A, Tilloy A, Chevalier S, Segretain D, and Pointis G (2008) Accelerated internalization of junctional membrane proteins (connexin 43, N-cadherin and ZO-1) within endocytic vacuoles: an early event of DDT carcinogenicity. Biochim Biophys Acta 1778:56-67.

Firouzi M, Bierhuizen MF, Kok B, Teunissen BE, Jansen AT, Jongsma HJ, and Groenewegen WA (2006) The human Cx40 promoter polymorphism -44G->A differentially affects transcriptional regulation by Sp1 and GATA4. Biochim Biophys Acta 1759:491-496.

Fishman GI, Moreno AP, Spray DC, and Leinwand LA (1991) Functional analysis of human cardiac gap junction channel mutants. Proc Natl Acad Sci USA 88 3525-3529.

Flenniken AM, Osborne LR, Anderson N, Ciliberti N, Fleming C, Gittens JE, Gong XQ, Kelsey LB, Lounsbury C, Moreno L, et al. (2005) A Gja1 missense mutation in a mouse model of oculodentodigital dysplasia. Development 132 4375-4386.

Fluri GS, Rüdisüli A, Willi M, Rohr S, and Weingart R (1990) Effects of arachidonic acid on the gap junctions of neonatal rat heart cells. Pflugers Arch 417:149-156.

Fong JT, Kells RM, Gumpert AM, Marzillier JY, Davidson MW, and Falk MM (2012) Internalized gap junctions are degraded by autophagy. Autophagy 8:794-811.
Fong JT, Kells RM, and Falk MM (2013) Two tyrosine-based sorting signals in the Cx43 C-terminus cooperate to mediate gap junction endocytosis. Mol Biol Cell $\mathbf{2 4}$ $2834-2848$

Foote CI, Zhou L, Zhu X, and Nicholson BJ (1998) The pattern of disulfide linkages in the extracellular loop regions of connexin 32 suggests a model for the docking interface of gap junctions. J Cell Biol 140:1187-1197.

Formigli L, Ibba-Manneschi L, Perna AM, Pacini A, Polidori L, Nediani C, Modesti PA, Nosi D, Tani A, Celli A, et al. (2003) Altered Cx43 expression during myocardial adaptation to acute and chronic volume overloading. Histol Histopathol 18: 359-369.

Freeman SM, Abboud CN, Whartenby KA, Packman CH, Koeplin DS, Moolten FL, and Abraham GN (1993) The "bystander effect": tumor regression when a fraction of the tumor mass is genetically modified. Cancer Res 53:5274-5283.

Freitas-Andrade M and Naus CC (2016) Astrocytes in neuroprotection and neurodegeneration: the role of connexin 43 and pannexin1. Neuroscience 323 $207-221$.

Froger N, Orellana JA, Calvo CF, Amigou E, Kozoriz MG, Naus CC, Sáez JC, and Giaume C (2010) Inhibition of cytokine-induced connexin43 hemichannel activity in astrocytes is neuroprotective. Mol Cell Neurosci 45:37-46.

Gaborit N, Steenman M, Lamirault G, Le Meur N, Le Bouter S, Lande G, Léger J, Charpentier F, Christ T, Dobrev D, et al. (2005) Human atrial ion channel and transporter subunit gene-expression remodeling associated with valvular heart disease and atrial fibrillation. Circulation 112:471-481.

Gaborit N, Varro A, Le Bouter S, Szuts V, Escande D, Nattel S, and Demolombe S (2010) Gender-related differences in ion-channel and transporter subunit expression in non-diseased human hearts. J Mol Cell Cardiol 49:639-646.

Gabriels JE and Paul DL (1998) Connexin43 is highly localized to sites of disturbed flow in rat aortic endothelium but connexin 37 and connexin 40 are more uniformly distributed. Circ Res 83:636-643.

Gadicherla AK, Wang N, Bulic M, Agullo-Pascual E, Lissoni A, De Smet M, Delmar M, Bultynck G, Krysko DV, Camara A, et al. (2017) Mitochondrial Cx43 hemichannels contribute to mitochondrial calcium entry and cell death in the heart. Basic Res Cardiol 112:27.

Gaietta G, Deerinck TJ, Adams SR, Bouwer J, Tour O, Laird DW, Sosinsky GE, Tsien RY, and Ellisman MH (2002) Multicolor and electron microscopic imaging of connexin trafficking. Science 296:503-507.

Gangoso E, Ezan P, Valle-Casuso JC, Herrero-González S, Koulakoff A, Medina JM Giaume C, and Tabernero A (2012) Reduced connexin43 expression correlates with c-Src activation, proliferation, and glucose uptake in reactive astrocytes after an excitotoxic insult. Glia 60:2040-2049.

Gangoso E, Thirant C, Chneiweiss H, Medina JM, and Tabernero A (2014) A cellpenetrating peptide based on the interaction between c-Src and connexin43 reverses glioma stem cell phenotype. Cell Death Dis 5:e1023.

Gao J, Sun X, Moore LC, White TW, Brink PR, and Mathias RT (2011) Lens intracellular hydrostatic pressure is generated by the circulation of sodium and modulated by gap junction coupling. J Gen Physiol 137:507-520.

Gao J, Liu RT, Cao S, Cui JZ, Wang A, To E, and Matsubara JA (2015) NLRP3 inflammasome: activation and regulation in age-related macular degeneration. Mediators Inflamm 2015:690243.

García-Dorado D, Rodríguez-Sinovas A, and Ruiz-Meana M (2004) Gap junctionmediated spread of cell injury and death during myocardial ischemia-reperfusion. Cardiovasc Res 61:386-401.

Garcia-Dorado D, Andres-Villarreal M, Ruiz-Meana M, Inserte J, and Barba I (2012) Myocardial edema: a translational view. J Mol Cell Cardiol 52:931-939.

GBD 2013 Mortality and Causes of Death Collaborators (2015) Global, regional, and national age-sex specific all-cause and cause-specific mortality for 240 causes of death, 1990-2013: a systematic analysis for the Global Burden of Disease Study 2013. Lancet 385:117-171.

Gemel J, Levy AE, Simon AR, Bennett KB, Ai X, Akhter S, and Beyer EC (2014a) Connexin40 abnormalities and atrial fibrillation in the human heart. $J$ Mol Cell Cardiol 76:159-168.

Gemel J, Simon AR, Patel D, Xu Q, Matiukas A, Veenstra RD, and Beyer EC (2014b) Degradation of a connexin40 mutant linked to atrial fibrillation is accelerated. $J$ Mol Cell Cardiol 74:330-339.

George SA and Poelzing S (2016) Cardiac conduction in isolated hearts of genetically modified mice-connexin43 and salts. Prog Biophys Mol Biol 120:189-198.

George SA, Bonakdar M, Zeitz M, Davalos RV, Smyth JW, and Poelzing S (2016) Extracellular sodium dependence of the conduction velocity-calcium relationship: evidence of ephaptic self-attenuation. Am J Physiol Heart Circ Physiol 310 H1129-H1139.

Ghatnekar GS, O'Quinn MP, Jourdan LJ, Gurjarpadhye AA, Draughn RL, and Gourdie RG (2009) Connexin43 carboxyl-terminal peptides reduce scar progenitor and promote regenerative healing following skin wounding. Regen Med 4 205-223.

Giardina SF, Mikami M, Goubaeva F, and Yang J (2007) Connexin 43 confers resistance to hydrogen peroxide-mediated apoptosis. Biochem Biophys Res Commun 362:747-752.

Giaume C, Koulakoff A, Roux L, Holcman D, and Rouach N (2010) Astroglial networks: a step further in neuroglial and gliovascular interactions. Nat Rev Neurosci 11:87-99.

Giaume C, Leybaert L, Naus CC, and Sáez JC (2013) Connexin and pannexin hemichannels in brain glial cells: properties, pharmacology, and roles. Front Pharmacol 4:88.

Giepmans BN (2004) Gap junctions and connexin-interacting proteins. Cardiovasc Res 62:233-245.

Giepmans BN, Verlaan I, Hengeveld T, Janssen H, Calafat J, Falk MM, and Moolenaar WH (2001) Gap junction protein connexin-43 interacts directly with microtubules. Curr Biol 11:1364-1368.

Gilleron J, Fiorini C, Carette D, Avondet C, Falk MM, Segretain D, and Pointis G (2008) Molecular reorganization of $\mathrm{Cx} 43, \mathrm{Zo}-1$ and Sre complexes during the 
endocytosis of gap junction plaques in response to a non-genomic carcinogen. $J$ Cell Sci 121:4069-4078.

Ginsberg M (1995) Neuroprotection in brain ischemia: an update. Neuroscientist 1: 95-103.

Girão H, Catarino S, and Pereira P (2009) Eps15 interacts with ubiquitinated Cx43 and mediates its internalization. Exp Cell Res 315:3587-3597.

Givvimani S, Pushpakumar S, Veeranki S, and Tyagi SC (2014) Dysregulation of Mfn2 and Drp-1 proteins in heart failure. Can J Physiol Pharmacol 92: 583-591.

Glass AM, Snyder EG, and Taffet SM (2015) Connexins and pannexins in the immune system and lymphatic organs. Cell Mol Life Sci 72:2899-2910.

Glukhov AV, Fedorov VV, Kalish PW, Ravikumar VK, Lou Q, Janks D, Schuessler RB, Moazami N, and Efimov IR (2012) Conduction remodeling in human end-stage nonischemic left ventricular cardiomyopathy. Circulation 125:1835-1847.

Goldberg GS, Moreno AP, Bechberger JF, Hearn SS, Shivers RR, MacPhee DJ, Zhang YC, and Naus CC (1996) Evidence that disruption of connexon particle arrangements in gap junction plaques is associated with inhibition of gap junctional communication by a glycyrrhetinic acid derivative. Exp Cell Res 222:48-53.

Goldberg GS, Moreno AP, and Lampe PD (2002) Gap junctions between cells expressing connexin 43 or 32 show inverse permselectivity to adenosine and ATP. $J$ Biol Chem 277:36725-36730.

Gollob MH, Jones DL, Krahn AD, Danis L, Gong XQ, Shao Q, Liu X, Veinot JP, Tang AS, Stewart AF, et al. (2006) Somatic mutations in the connexin 40 gene (GJA5) in atrial fibrillation. $N$ Engl J Med 354:2677-2688.

Gomes P, Srinivas SP, Van Driessche W, Vereecke J, and Himpens B (2005) ATP release through connexin hemichannels in corneal endothelial cells. Invest Ophthalmol Vis Sci 46:1208-1218.

Gómez-Hernández JM, de Miguel M, Larrosa B, González D, and Barrio LC (2003) Molecular basis of calcium regulation in connexin-32 hemichannels. Proc Natl Acad Sci USA 100:16030-16035.

Gong XQ and Nicholson BJ (2001) Size selectivity between gap junction channels composed of different connexins. Cell Commun Adhes 8:187-192.

Gong XQ, Shao Q, Lounsbury CS, Bai D, and Laird DW (2006) Functional characterization of a GJA1 frameshift mutation causing oculodentodigital dysplasia and palmoplantar keratoderma. J Biol Chem 281:31801-31811.

Gong XQ, Shao Q, Langlois S, Bai D, and Laird DW (2007) Differential potency of dominant negative connexin43 mutants in oculodentodigital dysplasia. $J$ Biol Chem 282:19190-19202.

González D, Gómez-Hernández JM, and Barrio LC (2007) Molecular basis of voltage dependence of connexin channels: an integrative appraisal. Prog Biophys Mol Biol 94:66-106.

González-Sánchez A, Jaraíz-Rodríguez M, Domínguez-Prieto M, Herrero-González S, Medina JM, and Tabernero A (2016) Connexin43 recruits PTEN and Csk to inhibit c-Src activity in glioma cells and astrocytes. Oncotarget 7:49819-49833.

Gonzalez JP, Ramachandran J, Xie LH, Contreras JE, and Fraidenraich D (2015) Selective connexin43 inhibition prevents isoproterenol-induced arrhythmias and lethality in muscular dystrophy mice. Sci Rep 5:13490.

Good ME, Begandt D, DeLalio LJ, Keller AS, Billaud M, and Isakson BE (2015) Emerging concepts regarding pannexin 1 in the vasculature. Biochem Soc Trans 43:495-501.

Goodenough DA (1974) Bulk isolation of mouse hepatocyte gap junctions. Characterization of the principal protein, connexin. J Cell Biol 61:557-563.

Goodenough DA and Revel JP (1970) A fine structural analysis of intercellular junctions in the mouse liver. $J$ Cell Biol 45:272-290.

Görbe A, Varga ZV, Kupai K, Bencsik P, Kocsis GF, Csont T, Boengler K, Schulz R, and Ferdinandy P (2011) Cholesterol diet leads to attenuation of ischemic preconditioning-induced cardiac protection: the role of connexin 43. Am J Physiol Heart Circ Physiol 300:H1907-H1913.

Gorlin RJ, Miskin LH, and St GEME JW (1963) Oculodentodigital dysplasia. $J$ Pediatr 63:69-75.

Gossman DG and Zhao HB (2008) Hemichannel-mediated inositol 1,4,5-trisphosphate (IP3) release in the cochlea: a novel mechanism of IP3 intercellular signaling. Cell Commun Adhes 15:305-315.

Gottwald E, Gottwald M, and Dhein S (1998) Enhanced dispersion of epicardial activation-recovery intervals at sites of histological inhomogeneity during regional cardiac ischaemia and reperfusion. Heart 79:474-480.

Goubaeva F, Mikami M, Giardina S, Ding B, Abe J, and Yang J (2007) Cardiac mitochondrial connexin 43 regulates apoptosis. Biochem Biophys Res Commun 352:97-103.

Green CR and Nicholson LF (2008) Interrupting the inflammatory cycle in chronic diseases-do gap junctions provide the answer? Cell Biol Int 32:1578-1583.

Grek CL, Prasad GM, Viswanathan V, Armstrong DG, Gourdie RG, and Ghatnekar GS (2015) Topical administration of a connexin43-based peptide augments healing of chronic neuropathic diabetic foot ulcers: a multicenter, randomized trial. Wound Repair Regen 23:203-212.

Groenewegen WA, Firouzi M, Bezzina CR, Vliex S, van Langen IM, Sandkuijl L, Smits JPP, Hulsbeek M, Rook MB, Jongsma HJ, et al. (2003) A cardiac sodium channel mutation cosegregates with a rare connexin40 genotype in familial atrial standstill. Circ Res 92:14-22.

Grover R and Dhein S (1998) Spatial structure determination of antiarrhythmic peptide using nuclear magnetic resonance spectroscopy. Peptides 19:1725-1729.

Grover R and Dhein S (2001) Structure-activity relationships of novel peptides related to the antiarrhythmic peptide AAP10 which reduce the dispersion of epicardial action potential duration. Peptides 22:1011-1021.

Grupcheva CN, Laux WT, Rupenthal ID, McGhee J, McGhee CN, and Green CR (2012) Improved corneal wound healing through modulation of gap junction communication using connexin43-specific antisense oligodeoxynucleotides. Invest Ophthalmol Vis Sci 53:1130-1138.

Guan J, Pavlovic D, Dalkie N, Waldvogel HJ, O'Carroll SJ, Green CR, and Nicholson LF (2013) Vascular degeneration in Parkinson's disease. Brain Pathol 23:154-164.
Guan X, Wilson S, Schlender KK, and Ruch RJ (1996) Gap-junction disassembly and connexin 43 dephosphorylation induced by 18 beta-glycyrrhetinic acid. $\mathrm{Mol}$ Carcinog 16:157-164.

Guerra JM, Everett TH IV, Lee KW, Wilson E, and Olgin JE (2006) Effects of the gap junction modifier rotigaptide (ZP123) on atrial conduction and vulnerability to atrial fibrillation. Circulation 114:110-118.

Guerrero PA, Schuessler RB, Davis LM, Beyer EC, Johnson CM, Yamada KA, and Saffitz JE (1997) Slow ventricular conduction in mice heterozygous for a connexin43 null mutation. J Clin Invest 99:1991-1998.

Gumpert AM, Varco JS, Baker SM, Piehl M, and Falk MM (2008) Double-membrane gap junction internalization requires the clathrin-mediated endocytic machinery. FEBS Lett 582:2887-2892.

Guo CX, Tran H, Green CR, Danesh-Meyer HV, and Acosta ML (2014) Gap junction proteins in the light-damaged albino rat. Mol Vis 20:670-682.

Guo CX, Mat Nor MN, Danesh-Meyer HV, Vessey KA, Fletcher EL, O'Carroll S.J, Acosta ML, and Green CR (2016) Connexin43 mimetic peptide improves retinal function and reduces inflammation in a light-damaged albino rat model. Invest Ophthalmol Vis Sci 57:3961-3973.

Guo S, Zhu J, Yang Z, Feng J, Li K, Wang R, and Yang X (2015) Reduction of connexin 37 expression by RNA interference decreases atherosclerotic plaque formation. Mol Med Rep 11:2664-2670.

Guo Y, Martinez-Williams C, Gilbert KA, and Rannels DE (1999) Inhibition of gap junction communication in alveolar epithelial cells by 18alpha-glycyrrhetinic acid Am J Physiol 276:L1018-L1026.

Gutstein DE, Morley GE, Tamaddon H, Vaidya D, Schneider MD, Chen J, Chien KR, Stuhlmann H, and Fishman GI (2001a) Conduction slowing and sudden arrhythmic death in mice with cardiac-restricted inactivation of connexin43. Circ Res 88: 333-339.

Gutstein DE, Morley GE, Vaidya D, Liu F, Chen FL, Stuhlmann H, and Fishman GI (2001b) Heterogeneous expression of Gap junction channels in the heart leads to conduction defects and ventricular dysfunction. Circulation 104:1194-1199.

Haefliger JA, Polikar R, Schnyder G, Burdet M, Sutter E, Pexieder T, Nicod P, and Meda $\mathrm{P}$ (2000) Connexin37 in normal and pathological development of mouse heart and great arteries. Dev Dyn 218:331-344.

Hagen A, Dietze A, and Dhein S (2009) Human cardiac gap-junction coupling: effects of antiarrhythmic peptide AAP10. Cardiovasc Res 83:405-415.

Hagendorff A, Schumacher B, Kirchhoff S, Lüderitz B, and Willecke K (1999) Conduction disturbances and increased atrial vulnerability in connexin40-deficient mice analyzed by transesophageal stimulation. Circulation 99:1508-1515.

Haleagrahara N, Varkkey J, and Chakravarthi S (2011) Cardioprotective effects of glycyrrhizic acid against isoproterenol-induced myocardial ischemia in rats. Int $J$ Mol Sci 12:7100-7113.

Han XJ, He D, Xu LJ, Chen M, Wang YQ, Feng JG, Wei MJ, Hong T, and Jiang LP (2015) Knockdown of connexin 43 attenuates balloon injury-induced vascular restenosis through the inhibition of the proliferation and migration of vascular smooth muscle cells. Int J Mol Med 36:1361-1368.

Han Y, Xi S, Zhang X, Yan C, Yang Y, and Kang J (2008) Association of connexin 37 gene polymorphisms with risk of coronary artery disease in northern Han Chinese. Cardiology 110:260-265.

Hansen DB, Braunstein TH, Nielsen MS, and MacAulay N (2014) Distinct permeation profiles of the connexin 30 and 43 hemichannels. FEBS Lett 588: $1446-1457$

Hansson GK, Libby P, and Tabas I (2015) Inflammation and plaque vulnerability. $J$ Intern Med 278:483-493.

Hare JF and Taylor K (1991) Mechanisms of plasma membrane protein degradation: recycling proteins are degraded more rapidly than those confined to the cell surface. Proc Natl Acad Sci USA 88:5902-5906.

Harks EG, de Roos AD, Peters PH, de Haan LH, Brouwer A, Ypey DL, van Zoelen EJ, and Theuvenet AP (2001) Fenamates: a novel class of reversible gap junction blockers. J Pharmacol Exp Ther 298:1033-1041.

Harris AL (2001) Emerging issues of connexin channels: biophysics fills the gap. $Q$ Rev Biophys 34:325-472.

Harris AL (2002) Voltage-sensing and substate rectification: moving parts of connexin channels. J Gen Physiol 119:165-169.

Harris AL (2007) Connexin channel permeability to cytoplasmic molecules. Prog Biophys Mol Biol 94:120-143.

Harris AL and Contreras JE (2014) Motifs in the permeation pathway of connexin channels mediate voltage and $\mathrm{Ca}^{(2+)}$ sensing. Front Physiol 5:113.

Hatanaka K, Kawata H, Toyofuku T, and Yoshida K (2004) Down-regulation of connexin43 in early myocardial ischemia and protective effect by ischemic preconditioning in rat hearts in vivo. Jpn Heart $J$ 45:1007-1019.

Hattori Y, Fukushima M, and Maitani Y (2007) Non-viral delivery of the connexin 43 gene with histone deacetylase inhibitor to human nasopharyngeal tumor cells enhances gene expression and inhibits in vivo tumor growth. Int $J$ Oncol 30: 1427-1439.

Hauer RN, Groenewegen WA, Firouzi M, Ramanna H, and Jongsma HJ (2006) Cx40 polymorphism in human atrial fibrillation. Adv Cardiol 42:284-291.

Haugan K, Lam HR, Knudsen CB, and Petersen JS (2004) Atrial fibrillation in rats induced by rapid transesophageal atrial pacing during brief episodes of asphyxia: a new in vivo model. $J$ Cardiovasc Pharmacol 44:125-135.

Haugan K, Olsen KB, Hartvig L, Petersen JS, Holstein-Rathlou NH, Hennan JK, and Nielsen MS (2005) The antiarrhythmic peptide analog ZP123 prevents atrial conduction slowing during metabolic stress. J Cardiovasc Electrophysiol 16: $537-545$.

Haugan K, Miyamoto T, Takeishi Y, Kubota I, Nakayama J, Shimojo H, and Hirose M (2006) Rotigaptide (ZP123) improves atrial conduction slowing in chronic volume overload-induced dilated atria. Basic Clin Pharmacol Toxicol 99:71-79.

Haussig S, Schubert A, Mohr FW, and Dhein S (2008) Sub-chronic nicotine exposure induces intercellular communication failure and differential down-regulation of connexins in cultured human endothelial cells. Atherosclerosis 196:210-218. 
Hawat G and Baroudi G (2008) Differential modulation of unapposed connexin 43 hemichannel electrical conductance by protein kinase $\mathrm{C}$ isoforms. Pflugers Arch 456:519-527.

Hawat G, Benderdour M, Rousseau G, and Baroudi G (2010) Connexin 43 mimetic peptide Gap26 confers protection to intact heart against myocardial ischemia injury. Pflugers Arch 460:583-592.

Hawat G, Hélie P, and Baroudi G (2012) Single intravenous low-dose injections of connexin 43 mimetic peptides protect ischemic heart in vivo against myocardial infarction. J Mol Cell Cardiol 53:559-566.

He DS and Burt JM (2000) Mechanism and selectivity of the effects of halothane on gap junction channel function. Circ Res 86:E104-E109.

He DS, Jiang JX, Taffet SM, and Burt JM (1999) Formation of heteromeric gap junction channels by connexins 40 and 43 in vascular smooth muscle cells. Proc Natl Acad Sci USA 96:6495-6500.

He H, Li N, Zhao Z, Han F, Wang X, and Zeng Y (2015) Ischemic postconditioning improves the expression of cellular membrane connexin 43 and attenuates the reperfusion injury in rat acute myocardial infarction. Biomed Rep 3:668-674.

Heinzel FR, Luo Y, Li X, Boengler K, Buechert A, García-Dorado D, Di Lisa F, Schulz $\mathrm{R}$, and Heusch G (2005) Impairment of diazoxide-induced formation of reactive oxygen species and loss of cardioprotection in connexin 43 deficient mice. Circ Res 97:583-586.

Hennan JK, Swillo RE, Morgan GA, Keith JC Jr, Schaub RG, Smith RP, Feldman HS, Haugan K, Kantrowitz J, Wang PJ, et al. (2006) Rotigaptide (ZP123) prevents spontaneous ventricular arrhythmias and reduces infarct size during myocardial ischemia/reperfusion injury in open-chest dogs. J Pharmacol Exp Ther $\mathbf{3 1 7}$ $236-243$.

Hernandez VH, Bortolozzi M, Pertegato V, Beltramello M, Giarin M, Zaccolo M, Pantano S, and Mammano F (2007) Unitary permeability of gap junction channels to second messengers measured by FRET microscopy. Nat Methods 4:353-358.

Hernández-Salinas R, Vielma AZ, Arismendi MN, Boric MP, Sáez JC, and Velarde V (2013) Boldine prevents renal alterations in diabetic rats. J Diabetes Res 2013 593672.

Herrero-González S, Valle-Casuso JC, Sánchez-Alvarez R, Giaume C, Medina JM, and Tabernero A (2009) Connexin43 is involved in the effect of endothelin-1 on astrocyte proliferation and glucose uptake. Glia 57:222-233.

Hertlein B, Butterweck A, Haubrich S, Willecke K, and Traub O (1998) Phosphorylated carboxy terminal serine residues stabilize the mouse gap junction protein connexin45 against degradation. J Membr Biol 162:247-257.

Hervé JC, Derangeon M, Sarrouilhe D, Giepmans BN, and Bourmeyster N (2012) Gap junctional channels are parts of multiprotein complexes. Biochim Biophys Acta 1818:1844-1865.

Hesketh GG, Shah MH, Halperin VL, Cooke CA, Akar FG, Yen TE, Kass DA, Machamer CE, Van Eyk JE, and Tomaselli GF (2010) Ultrastructure and regulation of lateralized connexin43 in the failing heart. Circ Res 106:1153-1163.

Heusch G, Büchert A, Feldhaus S, and Schulz R (2006) No loss of cardioprotection by postconditioning in connexin 43-deficient mice. Basic Res Cardiol 101: 354-356.

Hill MD, Martin RH, Mikulis D, Wong JH, Silver FL, Terbrugge KG, Milot G, Clark WM, Macdonald RL, Kelly ME, et al. (2012) Safety and efficacy of NA-1 in patients with iatrogenic stroke after endovascular aneurysm repair (ENACT): a phase 2, randomised, double-blind, placebo-controlled trial. Lancet Neurol 11:942-950.

Hirst-Jensen BJ, Sahoo P, Kieken F, Delmar M, and Sorgen PL (2007) Characterization of the $\mathrm{pH}$-dependent interaction between the gap junction protein connexin43 carboxyl terminus and cytoplasmic loop domains. J Biol Chem 282 $5801-5813$

Hofgaard JP, Banach K, Mollerup S, Jørgensen HK, Olesen SP, Holstein-Rathlou NH, and Nielsen MS (2008) Phosphatidylinositol-bisphosphate regulates in tercellular coupling in cardiac myocytes. Pflugers Arch 457:303-313.

Hofshi A, Itzhaki I, Gepstein A, Arbel G, Gross GJ, and Gepstein L (2011) A combined gene and cell therapy approach for restoration of conduction. Heart Rhythm 8 : $121-130$.

Homma N, Alvarado JL, Coombs W, Stergiopoulos K, Taffet SM, Lau AF, and Delmar M (1998) A particle-receptor model for the insulin-induced closure of connexin43 channels. Circ Res 83:27-32.

Hormuzdi SG, Pais I, LeBeau FE, Towers SK, Rozov A, Buhl EH, Whittington MA, and Monyer H (2001) Impaired electrical signaling disrupts gamma frequency oscillations in connexin 36-deficient mice. Neuron 31:487-495

Hossain MZ, Peeling J, Sutherland GR, Hertzberg EL, and Nagy JI (1994) Ischemiainduced cellular redistribution of the astrocytic gap junctional protein connexin43 in rat brain. Brain Res 652:311-322.

Hossmann KA (1994) Viability thresholds and the penumbra of focal ischemia. Ann Neurol 36:557-565

Hou J, Yan P, Guo T, Xing Y, Zheng S, Zhou C, Huang H, Long H, Zhong T, Wu Q, et al. (2015) Cardiac stem cells transplantation enhances the expression of connexin 43 via the ANG II/AT1R/TGF-beta1 signaling pathway in a rat model of myocardial infarction. Exp Mol Pathol 99:693-701.

Howarth FC, Chandler NJ, Kharche S, Tellez JO, Greener ID, Yamanushi TT, Billeter R, Boyett MR, Zhang H, and Dobrzynski H (2008) Effects of streptozotocin-induced diabetes on connexin43 mRNA and protein expression in ventricular muscle. Mol Cell Biochem 319:105-114.

Hu Y, Chen IP, de Almeida S, Tiziani V, Do Amaral CM, Gowrishankar K, PassosBueno MR, and Reichenberger EJ (2013) A novel autosomal recessive GJA1 missense mutation linked to craniometaphyseal dysplasia. PLoS One 8:e73576.

Huang C, Han X, Li X, Lam E, Peng W, Lou N, Torres A, Yang M, Garre JM, Tian GF, et al. (2012a) Critical role of connexin 43 in secondary expansion of traumatic spinal cord injury. J Neurosci 32:3333-3338.

Huang J, Wang D, Zheng J, Huang X, and Jin H (2012b) Hydrogen sulfide attenuates cardiac hypertrophy and fibrosis induced by abdominal aortic coarctation in rats. Mol Med Rep 5:923-928.
Huang T, Shao Q, Barr K, Simek J, Fishman GI, and Laird DW (2014) Myogenic bladder defects in mouse models of human oculodentodigital dysplasia. Biochem $J$ 457:441-449.

Huang YS, Tseng YZ, Wu JC, and Wang SM (2004) Mechanism of oleic acid-induced gap junctional disassembly in rat cardiomyocytes. $\mathrm{J} \mathrm{Mol} \mathrm{Cell} \mathrm{Cardiol} \mathrm{37:755-766.}$

Hubbard ML, Ying W, and Henriquez CS (2007) Effect of gap junction distribution on impulse propagation in a monolayer of myocytes: a model study. Europace $\mathbf{9}$ (Suppl 6):vi20-vi28.

Hulsmans M, Clauss S, Xiao L, Aguirre AD, King KR, Hanley A, Hucker WJ, Wulfers EM, Seemann G, Courties G, et al. (2017) Macrophages facilitate electrical conduction in the heart. Cell 169:510-522 e520.

Hund TJ, Lerner DL, Yamada KA, Schuessler RB, and Saffitz JE (2007) Protein kinase cepsilon mediates salutary effects on electrical coupling induced by ischemic preconditioning. Heart Rhythm 4:1183-1193.

Hunter AW, Jourdan J, and Gourdie RG (2003) Fusion of GFP to the carboxyl terminus of connexin 43 increases gap junction size in HeLa cells. Cell Commun Adhes 10:211-214.

Hunter AW, Barker RJ, Zhu C, and Gourdie RG (2005) Zonula occludens-1 alters connexin43 gap junction size and organization by influencing channel accretion. Mol Biol Cell 16:5686-5698.

Hussain W, Patel PM, Chowdhury RA, Cabo C, Ciaccio EJ, Lab MJ, Duffy HS, Wit $\mathrm{AL}$, and Peters NS (2010) The renin-angiotensin system mediates the effects of stretch on conduction velocity, connexin43 expression, and redistribution in intact ventricle. J Cardiovasc Electrophysiol 21:1276-1283.

Inoue H, Mori T, Shibata S, and Koshihara Y (1989) Modulation by glycyrrhetinic acid derivatives of TPA-induced mouse ear oedema. Br J Pharmacol 96:204-210.

Iwasaki YK, Nishida K, Kato T, and Nattel S (2011) Atrial fibrillation pathophysiology: implications for management. Circulation 124:2264-2274.

Iyyathurai J, D'hondt C, Wang N, De Bock M, Himpens B, Retamal MA, Stehberg J, Leybaert L, and Bultynck G (2013) Peptides and peptide-derived molecules targeting the intracellular domains of $\mathrm{Cx} 43$ : gap junctions versus hemichannels. Neuropharmacology 75:491-505.

Iyyathurai J, Decuypere JP, Leybaert L, D'hondt C, and Bultynck G (2016) Connexins: substrates and regulators of autophagy. BMC Cell Biol 17 (Suppl 1):20 Jackson PE, Feng QP, and Jones DL (2008) Nitric oxide depresses connexin 43 after myocardial infarction in mice. Acta Physiol (Oxf) 194:23-33.

Jain SK, Schuessler RB, and Saffitz JE (2003) Mechanisms of delayed electrical uncoupling induced by ischemic preconditioning. Circ Res 92:1138-1144.

Janota C, Lemere CA, and Brito MA (2016) Dissecting the contribution of vascular alterations and aging to Alzheimer's disease. Mol Neurobiol 53:3793-3811.

Janse MJ (1986) Electrophysiological effects of myocardial ischaemia. Relationship with early ventricular arrhythmias. Eur Heart J 7 (Suppl A):35-43.

Janse MJ, van Capelle FJ, Morsink H, Kléber AG, Wilms-Schopman F, Cardinal R d'Alnoncourt CN, and Durrer D (1980) Flow of "injury" current and patterns of excitation during early ventricular arrhythmias in acute regional myocardial ischemia in isolated porcine and canine hearts. Evidence for two different arrhythmogenic mechanisms. Circ Res 47:151-165.

Janse MJ, Kleber AG, Capucci A, Coronel R, and Wilms-Schopman F (1986) Electrophysiological basis for arrhythmias caused by acute ischemia. Role of the subendocardium. J Mol Cell Cardiol 18:339-355.

Jansen JA, Noorman M, Musa H, Stein M, de Jong S, van der Nagel R, Hund TJ, Mohler PJ, Vos MA, van Veen TA, et al. (2012) Reduced heterogeneous expression of $\mathrm{Cx} 43$ results in decreased Nav1.5 expression and reduced sodium current that accounts for arrhythmia vulnerability in conditional $\mathrm{Cx} 43$ knockout mice. Heart Rhythm 9:600-607.

Jaraíz-Rodríguez M, Tabernero MD, González-Tablas M, Otero A, Orfao A, Medina JM, and Tabernero A (2017) A short region of connexin43 reduces human glioma stem cell migration, invasion and survival through Src, PTEN and FAK. Stem Cell Rep DOI: 10.1016/j.stemcr.2017.06.007 [published ahead of print].

Jiang JX and Goodenough DA (1998) Phosphorylation of lens-fiber connexins in lens organ cultures. Eur J Biochem 255:37-44.

Jiang JX and Gu S (2005) Gap junction- and hemichannel-independent actions of connexins. Biochim Biophys Acta 1711:208-214

Jin C, Martyn KD, Kurata WE, Warn-Cramer BJ, and Lau AF (2004) Connexin43 PDZ2 binding domain mutants create functional gap junctions and exhibit altered phosphorylation. Cell Commun Adhes 11:67-87.

Johansen D, Cruciani V, Sundset R, Ytrehus K, and Mikalsen SO (2011) Ischemia induces closure of gap junctional channels and opening of hemichannels in heartderived cells and tissue. Cell Physiol Biochem 28:103-114.

John S, Cesario D, and Weiss JN (2003) Gap junctional hemichannels in the heart. Acta Physiol Scand 179:23-31.

Johnson KE, Mitra S, Katoch P, Kelsey LS, Johnson KR, and Mehta PP (2013) Phosphorylation on Ser-279 and Ser-282 of connexin43 regulates endocytosis and gap junction assembly in pancreatic cancer cells. Mol Biol Cell 24:715-733.

Johnson RG, Meyer RA, Li XR, Preus DM, Tan L, Grunenwald H, Paulson AF, Laird DW, and Sheridan JD (2002) Gap junctions assemble in the presence of cytoskeletal inhibitors, but enhanced assembly requires microtubules. Exp Cell Res 275 $67-80$

Johnstone SR, Ross J, Rizzo MJ, Straub AC, Lampe PD, Leitinger N, and Isakson BE (2009) Oxidized phospholipid species promote in vivo differential cx43 phosphorylation and vascular smooth muscle cell proliferation. Am J Pathol 175:916-924. Johnstone SR, Kroncke BM, Straub AC, Best AK, Dunn CA, Mitchell LA, Peskova Y, Nakamoto RK, Koval M, Lo CW, et al. (2012) MAPK phosphorylation of connexin 43 promotes binding of cyclin $\mathrm{E}$ and smooth muscle cell proliferation. Circ Res 111: 201-211.

Jones SA and Lancaster MK (2015) Progressive age-associated activation of JNK associates with conduction disruption in the aged atrium. Mech Ageing Dev 146148:72-80.

Jones SA, Lancaster MK, and Boyett MR (2004) Ageing-related changes of connexins and conduction within the sinoatrial node. J Physiol 560:429-437. 
Jongen WM, Fitzgerald DJ, Asamoto M, Piccoli C, Slaga TJ, Gros D, Takeichi M, and Yamasaki H (1991) Regulation of connexin 43-mediated gap junctional in tercellular communication by $\mathrm{Ca}^{2+}$ in mouse epidermal cells is controlled by E-cadherin. J Cell Biol 114:545-555.

Jordan K, Solan JL, Dominguez M, Sia M, Hand A, Lampe PD, and Laird DW (1999) Trafficking, assembly, and function of a connexin43-green fluorescent protein chimera in live mammalian cells. Mol Biol Cell 10:2033-2050.

Jordan K, Chodock R, Hand AR, and Laird DW (2001) The origin of annular junctions: a mechanism of gap junction internalization. J Cell Sci 114:763-773.

Jørgensen NR, Teilmann SC, Henriksen Z, Meier E, Hansen SS, Jensen JE, Sørensen $\mathrm{OH}$, and Petersen JS (2005) The antiarrhythmic peptide analog rotigaptide (ZP123) stimulates gap junction intercellular communication in human osteoblasts and prevents decrease in femoral trabecular bone strength in ovariectomized rats. Endocrinology 146:4745-4754.

Joshi MS, Mihm MJ, Cook AC, Schanbacher BL, and Bauer JA (2015) Alterations in connexin 43 during diabetic cardiomyopathy: competition of tyrosine nitration versus phosphorylation. J Diabetes 7:250-259.

Jovic M, Stancic A, Nenadic D, Cekic O, Nezic D, Milojevic P, Micovic S, Buzadzic B, Korac A, Otasevic V, et al. (2012) Mitochondrial molecular basis of sevoflurane and propofol cardioprotection in patients undergoing aortic valve replacement with cardiopulmonary bypass. Cell Physiol Biochem 29:131-142.

Joyner RW and van Capelle FJ (1986) Propagation through electrically coupled cells. How a small SA node drives a large atrium. Biophys $J$ 50:1157-1164.

Jozwiak J and Dhein S (2008) Local effects and mechanisms of antiarrhythmic peptide AAP10 in acute regional myocardial ischemia: electrophysiological and molecular findings. Naunyn Schmiedebergs Arch Pharmacol 378 $459-470$

Jozwiak J, Dietze A, Grover R, Savtschenko A, Etz C, Mohr FW, and Dhein S (2012) Desipramine prevents cardiac gap junction uncoupling. Naunyn Schmiedebergs Arch Pharmacol 385:1063-1075.

Kalcheva N, Qu J, Sandeep N, Garcia L, Zhang J, Wang Z, Lampe PD, Suadicani SO, Spray DC, and Fishman GI (2007) Gap junction remodeling and cardiac arrhythmogenesis in a murine model of oculodentodigital dysplasia. Proc Natl Acad Sci USA 104:20512-20516.

Kalma Y, Granot I, Galiani D, Barash A, and Dekel N (2004) Luteinizing hormoneinduced connexin 43 down-regulation: inhibition of translation. Endocrinology 145 $1617-1624$.

Kanagaratnam P, Cherian A, Stanbridge RD, Glenville B, Severs NJ, and Peters NS (2004) Relationship between connexins and atrial activation during human atrial fibrillation. J Cardiovasc Electrophysiol 15:206-216.

Kanaporis G, Brink PR, and Valiunas V (2011) Gap junction permeability: selectivity for anionic and cationic probes. Am J Physiol Cell Physiol 300:C600-C609.

Kanczuga-Koda L, Sulkowski S, Koda M, Skrzydlewska E, and Sulkowska M (2005a) Connexin 26 correlates with Bcl-xL and Bax proteins expression in colorectal cancer. World J Gastroenterol 11:1544-1548.

Kanczuga-Koda L, Sulkowski S, Tomaszewski J, Koda M, Sulkowska M, Przystupa W, Golaszewska J, and Baltaziak M (2005b) Connexins 26 and 43 correlate with Bak, but not with Bcl-2 protein in breast cancer. Oncol Rep 14:325-329.

Kanemitsu MY and Lau AF (1993) Epidermal growth factor stimulates the disruption of gap junctional communication and connexin 43 phosphorylation independent of 12-0-tetradecanoylphorbol 13-acetate-sensitive protein kinase C: the possible involvement of mitogen-activated protein kinase. Mol Biol Cell 4: $837-848$

Kanemitsu MY, Loo LW, Simon S, Lau AF, and Eckhart W (1997) Tyrosine phosphorylation of connexin 43 by v-Src is mediated by SH2 and SH3 domain interactions. $J$ Biol Chem 272:22824-22831.

Kanemitsu MY, Jiang W, and Eckhart W (1998) Cdc2-mediated phosphorylation of the gap junction protein, connexin43, during mitosis. Cell Growth Differ 9:13-21.

Kang J, Kang N, Lovatt D, Torres A, Zhao Z, Lin J, and Nedergaard M (2008) Connexin 43 hemichannels are permeable to ATP. J Neurosci 28:4702-4711.

Kanneganti TD, Lamkanfi M, Kim YG, Chen G, Park JH, Franchi L, Vandenabeele $P$, and Núñez G (2007) Pannexin-1-mediated recognition of bacterial molecules activates the cryopyrin inflammasome independent of Toll-like receptor signaling. Immunity 26:433-443.

Kanno S, Kovacs A, Yamada KA, and Saffitz JE (2003) Connexin43 as a determinant of myocardial infarct size following coronary occlusion in mice. J Am Coll Cardiol 41:681-686.

Kaplan SR, Gard JJ, Protonotarios N, Tsatsopoulou A, Spiliopoulou C, Anastasakis A, Squarcioni CP, McKenna WJ, Thiene G, Basso C, et al. (2004) Remodeling of myocyte gap junctions in arrhythmogenic right ventricular cardiomyopathy due to a deletion in plakoglobin (Naxos disease). Heart Rhythm 1:3-11.

Karagiannis A, Sylantyev S, Hadjihambi A, Hosford PS, Kasparov S, and Gourine AV (2016) Hemichannel-mediated release of lactate. J Cereb Blood Flow Metab $\mathbf{3 6}$ 1202-1211.

Karagueuzian HS, Sugi K, Ohta M, Fishbein MC, Mandel WJ, and Peter T (1986) Inducible sustained ventricular tachycardia and ventricular fibrillation in conscious dogs with isolated right ventricular infarction: relation to infarct structure. J Am Coll Cardiol 7:850-858.

Karagueuzian HS, Athill CA, Yashima M, Ikeda T, Wu TJ, Mandel WJ, and Chen PS (1998) Transmembrane potential properties of atrial cells at different sites of a spiral wave reentry: cellular evidence for an excitable but nonexcited core. Pacing Clin Electrophysiol 21:2360-2365.

Kato T, Iwasaki YK, and Nattel S (2012) Connexins and atrial fibrillation: filling in the gaps. Circulation 125:203-206.

Kaur C, Foulds WS, and Ling EA (2008) Blood-retinal barrier in hypoxic ischaemic conditions: basic concepts, clinical features and management. Prog Retin Eye Res 27:622-647.

Kausalya PJ, Reichert M, and Hunziker W (2001) Connexin45 directly binds to ZO-1 and localizes to the tight junction region in epithelial MDCK cells. FEBS Lett $\mathbf{5 0 5}$ : 92-96.
Kelly JJ, Shao Q, Jagger DJ, and Laird DW (2015) Cx30 exhibits unique characteristics including a long half-life when assembled into gap junctions. J Cell Sci 128:3947-3960.

Kelly JJ, Esseltine JL, Shao Q, Jabs EW, Sampson J, Auranen M, Bai D, and Laird DW (2016) Specific functional pathologies of $\mathrm{Cx} 43$ mutations associated with oculodentodigital dysplasia. Mol Biol Cell 27:2172-2185.

Kelly SM, Vanslyke JK, and Musil LS (2007) Regulation of ubiquitin-proteasome system mediated degradation by cytosolic stress. Mol Biol Cell 18:4279-4291.

Kieken F, Mutsaers N, Dolmatova E, Virgil K, Wit AL, Kellezi A, Hirst-Jensen BJ, Duffy HS, and Sorgen PL (2009) Structural and molecular mechanisms of gap junction remodeling in epicardial border zone myocytes following myocardial infarction. Circ Res 104:1103-1112.

Kielian T (2008) Glial connexins and gap junctions in CNS inflammation and disease. $J$ Neurochem 106:1000-1016.

Kienitz MC, Bender K, Dermietzel R, Pott L, and Zoidl G (2011) Pannexin 1 constitutes the large conductance cation channel of cardiac myocytes. J Biol Chem 286: $290-298$

Kim DY, Kam Y, Koo SK, and Joe CO (1999) Gating connexin 43 channels reconstituted in lipid vesicles by mitogen-activated protein kinase phosphorylation. $J$ Biol Chem 274:5581-5587.

Kim MS, Zhong J, and Pandey A (2016a) Common errors in mass spectrometry-based analysis of post-translational modifications. Proteomics 16:700-714.

Kim Y and Green CR(2016) Assessing connexin hemichannel function during ischemic injury and reperfusion, in Gap Junction Channels and Hemichannels - Meth ods in Signal Transduction Series (Bai D and Saez JC eds), CRC Press, Taylor \& Francis Group, Boca Raton.

Kim Y, Davidson JO, Gunn KC, Phillips AR, Green CR, and Gunn AJ (2016b) Role of Hemichannels in CNS Inflammation and the Inflammasome Pathway. Adv Protein Chem Struct Biol 104:1-37.

Kim Y, Griffin JM, Harris PW, Chan SH, Nicholson LF, Brimble MA, O'Carroll SJ, and Green CR (2017) Characterizing the mode of action of extracellular connexin43 channel blocking mimetic peptides in an in vitro ischemia injury model. Biochim Biophys Acta 1861:68-78.

Kirca M, Kleinbongard P, Soetkamp D, Heger J, Csonka C, Ferdinandy P, and Schulz $\mathrm{R}$ (2015) Interaction between connexin 43 and nitric oxide synthase in mice heart mitochondria. $J$ Cell Mol Med 19:815-825.

Kirchhoff S, Nelles E, Hagendorff A, Krüger O, Traub O, and Willecke K (1998) Reduced cardiac conduction velocity and predisposition to arrhythmias in connexin40-deficient mice. Curr Biol 8:299-302.

Kitamura H, Ohnishi Y, Yoshida A, Okajima K, Azumi H, Ishida A, Galeano EJ, Kubo S, Hayashi Y, Itoh H, et al. (2002) Heterogeneous loss of connexin43 protein in nonischemic dilated cardiomyopathy with ventricular tachycardia. J Cardiovasc Electrophysiol 13:865-870.

Kjenseth A, Fykerud TA, Sirnes S, Bruun J, Yohannes Z, Kolberg M, Omori Y, Rivedal E, and Leithe E (2012) The gap junction channel protein connexin 43 is covalently modified and regulated by SUMOylation. $J$ Biol Chem 287: 15851-15861.

Kjølbye AL, Knudsen CB, Jepsen T, Larsen BD, and Petersen JS (2003) Pharmacological characterization of the new stable antiarrhythmic peptide analog Ac-D-TyrD-Pro-D-Hyp-Gly-D-Ala-Gly-NH2 (ZP123): in vivo and in vitro studies. $J$ Pharmacol Exp Ther 306:1191-1199.

Kjølbye AL, Haugan K, Hennan JK, and Petersen JS (2007) Pharmacological modulation of gap junction function with the novel compound rotigaptide: a promising new principle for prevention of arrhythmias. Basic Clin Pharmacol Toxicol 101: $215-230$.

Kjølbye AL, Dikshteyn M, Eloff BC, Deschênes I, and Rosenbaum DS (2008) Maintenance of intercellular coupling by the antiarrhythmic peptide rotigaptide suppresses arrhythmogenic discordant alternans. Am J Physiol Heart Circ Physio 294:H41-H49.

Klaunig JE and Shi Y(2009) Assessment of gap junctional intercellular communication. Curr Protoc Toxicol Chapter 2:Unit2 17.

Kléber G (1992) The potential role of $\mathrm{Ca}^{2+}$ for electrical cell-to-cell uncoupling and conduction block in myocardial tissue. Basic Res Cardiol 87 (Suppl 2):131-143.

Knezl V, Bacova B, Kolenova L, Mitasikova M, Weismann P, Drimal J, and Tribulova N (2008) Distinct lethal arrhythmias susceptibility is associated with sex-related difference in myocardial connexin-43 expression. Neuroendocrinol Lett 29:798-801.

Kojima T, Kokai Y, Chiba H, Yamamoto M, Mochizuki Y, and Sawada N (2001) Cx32 but not $\mathrm{Cx} 26$ is associated with tight junctions in primary cultures of rat hepatocytes. Exp Cell Res 263:193-201.

Kondo RP, Wang SY, John SA, Weiss JN, and Goldhaber JI (2000) Metabolic inhibition activates a non-selective current through connexin hemichannels in isolated ventricular myocytes. J Mol Cell Cardiol 32:1859-1872.

Kostin S (2007) Zonula occludens-1 and connexin 43 expression in the failing human heart. J Cell Mol Med 11:892-895.

Kostin S, Klein G, Szalay Z, Hein S, Bauer EP, and Schaper J (2002) Structural correlate of atrial fibrillation in human patients. Cardiovasc Res 54:361-379.

Kostin S, Rieger M, Dammer S, Hein S, Richter M, Klövekorn WP, Bauer EP, and Schaper J (2003) Gap junction remodeling and altered connexin43 expression in the failing human heart. Mol Cell Biochem 242:135-144.

Kostin S, Dammer S, Hein S, Klovekorn WP, Bauer EP, and Schaper J (2004) Connexin 43 expression and distribution in compensated and decompensated cardiac hypertrophy in patients with aortic stenosis. Cardiovasc Res 62:426-436.

Kothmann WW, Li X, Burr GS, and O’Brien J (2007) Connexin 35/36 is phosphorylated at regulatory sites in the retina. Vis Neurosci 24:363-375.

Kothmann WW, Massey SC, and O'Brien J (2009) Dopamine-stimulated dephosphorylation of connexin 36 mediates AII amacrine cell uncoupling. $J$ Neurosci 29:14903-14911.

Koulakoff A, Ezan P, and Giaume C (2008) Neurons control the expression of connexin 30 and connexin 43 in mouse cortical astrocytes. Glia 56:1299-1311. 
Koura T, Hara M, Takeuchi S, Ota K, Okada Y, Miyoshi S, Watanabe A, Shiraiwa K, Mitamura H, Kodama I, et al. (2002) Anisotropic conduction properties in canine atria analyzed by high-resolution optical mapping: preferential direction of conduction block changes from longitudinal to transverse with increasing age. Circulation 105:2092-2098.

Koval M, Harley JE, Hick E, and Steinberg TH (1997) Connexin46 is retained as monomers in a trans-Golgi compartment of osteoblastic cells. J Cell Biol 137: $847-857$.

Koval M, Molina SA, and Burt JM (2014) Mix and match: investigating heteromeric and heterotypic gap junction channels in model systems and native tissues. FEBS Lett 588:1193-1204.

Kozoriz MG, Bechberger JF, Bechberger GR, Suen MW, Moreno AP, Maass K, Willecke K, and Naus CC (2010) The connexin43 C-terminal region mediates neuroprotection during stroke. J Neuropathol Exp Neurol 69:196-206.

Kozoriz MG, Lai S, Vega JL, Sáez JC, Sin WC, Bechberger JF, and Naus CC (2013) Cerebral ischemic injury is enhanced in a model of oculodentodigital dysplasia. Neuropharmacology 75:549-556.

Kreuzberg MM, Söhl G, Kim JS, Verselis VK, Willecke K, and Bukauskas FF (2005) Functional properties of mouse connexin30.2 expressed in the conduction system of the heart. Circ Res 96:1169-1177.

Kreuzberg MM, Schrickel JW, Ghanem A, Kim JS, Degen J, Janssen-Bienhold U, Lewalter T, Tiemann K, and Willecke K (2006) Connexin30.2 containing gap junction channels decelerate impulse propagation through the atrioventricular node. Proc Natl Acad Sci USA 103:5959-5964.

Kreuzberg MM, Deuchars J, Weiss E, Schober A, Sonntag S, Wellershaus K, Draguhn A, and Willecke K (2008) Expression of connexin30.2 in interneurons of the central nervous system in the mouse. Mol Cell Neurosci 37:119-134.

Krinsky VI (1978) Mathematical models of cardiac arrhythmias (spiral waves). Pharmacol Ther [B] 3:539-555.

Kwak BR and Jongsma HJ (1996) Regulation of cardiac gap junction channel permeability and conductance by several phosphorylating conditions. Mol Cell Biochem 157:93-99.

Kwak BR and Jongsma HJ (1999) Selective inhibition of gap junction channel activity by synthetic peptides. J Physiol 516:679-685.

Kwak BR, Hermans MM, De Jonge HR, Lohmann SM, Jongsma HJ, and Chanson M (1995a) Differential regulation of distinct types of gap junction channels by similar phosphorylating conditions. Mol Biol Cell 6:1707-1719.

Kwak BR, Sáez JC, Wilders R, Chanson M, Fishman GI, Hertzberg EL, Spray DC, and Jongsma HJ (1995b) Effects of cGMP-dependent phosphorylation on rat and human connexin43 gap junction channels. Pflugers Arch 430:770-778.

Kwak BR, van Veen TA, Analbers LJ, and Jongsma HJ (1995c) TPA increases conductance but decreases permeability in neonatal rat cardiomyocyte gap junction channels. Exp Cell Res 220:456-463.

Kwak BR, Mulhaupt F, Veillard N, Gros DB, and Mach F (2002) Altered pattern of vascular connexin expression in atherosclerotic plaques. Arterioscler Thromb Vasc Biol 22:225-230.

Kwak BR, Veillard N, Pelli G, Mulhaupt F, James RW, Chanson M, and Mach F (2003) Reduced connexin43 expression inhibits atherosclerotic lesion formation in low-density lipoprotein receptor-deficient mice. Circulation 107:1033-1039.

Kwak BR, Silacci P, Stergiopulos N, Hayoz D, and Meda P (2005) Shear stress and cyclic circumferential stretch, but not pressure, alter connexin43 expression in endothelial cells. Cell Commun Adhes 12:261-270.

Kwak BR, Back M, Bochaton-Piallat ML, Caligiuri G, Daemen MJ, Davies PF, Hoefer IE, Holvoet P, Jo H, Krams R, Lehoux S, Monaco C, Steffens S, Virmani R, Weber C, Wentzel JJ, and Evans PC (2014) Biomechanical factors in atherosclerosis: mechanisms and clinical implications. Eur Heart $J$ 35:3013-3020, 3020a-3020d.

Lai A, Le DN, Paznekas WA, Gifford WD, Jabs EW, and Charles AC (2006) Oculodentodigital dysplasia connexin43 mutations result in non-functional connexin hemichannels and gap junctions in C6 glioma cells. J Cell Sci 119:532-541.

Laing JG and Beyer EC (1995) The gap junction protein connexin43 is degraded via the ubiquitin proteasome pathway. J Biol Chem 270:26399-26403.

Laing JG, Tadros PN, Westphale EM, and Beyer EC (1997) Degradation of connexin43 gap junctions involves both the proteasome and the lysosome. Exp Cell Res 236:482-492

Laing JG, Tadros PN, Green K, Saffitz JE, and Beyer EC (1998) Proteolysis of connexin43-containing gap junctions in normal and heat-stressed cardiac myocytes. Cardiovasc Res 38:711-718.

Laing JG, Manley-Markowski RN, Koval M, Civitelli R, and Steinberg TH (2001) Connexin 45 interacts with zonula occludens- 1 and connexin 43 in osteoblastic cells. $J$ Biol Chem 276:23051-23055.

Laird DW (2006) Life cycle of connexins in health and disease. Biochem $J$ 394: $527-543$

Laird DW (2008) Closing the gap on autosomal dominant connexin-26 and connexin43 mutants linked to human disease. J Biol Chem 283:2997-3001.

Laird DW (2010) The gap junction proteome and its relationship to disease. Trends Cell Biol 20:92-101.

Laird DW (2014) Syndromic and non-syndromic disease-linked Cx43 mutations. FEBS Lett 588:1339-1348.

Laird DW and Revel JP (1990) Biochemical and immunochemical analysis of the arrangement of connexin43 in rat heart gap junction membranes. J Cell Sci 97: 109-117.

Laird DW, Puranam KL, and Revel JP (1991) Turnover and phosphorylation dynamics of connexin43 gap junction protein in cultured cardiac myocytes. Biochem $J$ 273:67-72.

Lakkisto P, Csonka C, Fodor G, Bencsik P, Voipio-Pulkki LM, Ferdinandy P, and Pulkki K (2009) The heme oxygenase inducer hemin protects against cardiac dysfunction and ventricular fibrillation in ischaemic/reperfused rat hearts: role of connexin 43. Scand J Clin Lab Invest 69:209-218.

Lamiche C, Clarhaut J, Strale PO, Crespin S, Pedretti N, Bernard FX, Naus CC, Chen VC, Foster LJ, Defamie N, et al. (2012) The gap junction protein $\mathrm{Cx} 43$ is involved in the bone-targeted metastatic behaviour of human prostate cancer cells. Clin Exp Metastasis 29:111-122.

Lampe PD (1994) Analyzing phorbol ester effects on gap junctional communication: a dramatic inhibition of assembly. J Cell Biol 127:1895-1905.

Lampe PD and Lau AF (2000) Regulation of gap junctions by phosphorylation of connexins. Arch Biochem Biophys 384:205-215.

Lampe PD, Kurata WE, Warn-Cramer BJ, and Lau AF (1998) Formation of a distinct connexin 43 phosphoisoform in mitotic cells is dependent upon p34cdc2 kinase. $J$ Cell Sci 111:833-841.

Lampe PD, TenBroek EM, Burt JM, Kurata WE, Johnson RG, and Lau AF (2000) Phosphorylation of connexin43 on serine 368 by protein kinase $\mathrm{C}$ regulates gap junctional communication. J Cell Biol 149:1503-1512.

Lampe PD, Cooper CD, King TJ, and Burt JM (2006) Analysis of Connexin43 phosphorylated at S325, S328 and S330 in normoxic and ischemic heart. J Cell Sci 119:3435-3442.

Lancaster TS, Jefferson SJ, and Korzick DH (2011) Local delivery of a PKC $\varepsilon$ activating peptide limits ischemia reperfusion injury in the aged female rat heart. Am J Physiol Regul Integr Comp Physiol 301:R1242-R1249.

Lane NJ and Swales LS (1980) Dispersal of junctional particles, not internalization, during the in vivo disappearance of gap junctions. Cell 19:579-586.

Larsen MR, Trelle MB, Thingholm TE, and Jensen ON (2006) Analysis of posttranslational modifications of proteins by tandem mass spectrometry. Biotechniques 40:790-798.

Larson DM, Christensen TG, Sagar GD, and Beyer EC (2001) TGF-beta1 induces an accumulation of connexin 43 in a lysosomal compartment in endothelial cells. Endothelium 8:255-260

Lau AF (2005) c-Src: bridging the gap between phosphorylation- and acidificationinduced gap junction channel closure. Sci STKE 2005:pe33.

Lauf U, Giepmans BN, Lopez P, Braconnot S, Chen SC, and Falk MM (2002) Dynamic trafficking and delivery of connexons to the plasma membrane and accretion to gap junctions in living cells. Proc Natl Acad Sci USA 99:10446-10451.

Lazrak A and Peracchia C (1993) Gap junction gating sensitivity to physiological internal calcium regardless of $\mathrm{pH}$ in Novikoff hepatoma cells. Biophys $J \mathbf{6 5}$ : 2002-2012

Lazrak A, Peres A, Giovannardi S, and Peracchia C (1994) Ca-mediated and independent effects of arachidonic acid on gap junctions and Ca-independent effects of oleic acid and halothane. Biophys $J$ 67:1052-1059.

Le HT, Sin WC, Lozinsky S, Bechberger J, Vega JL, Guo XQ, Sáez JC, and Naus CC (2014) Gap junction intercellular communication mediated by connexin43 in astrocytes is essential for their resistance to oxidative stress. J Biol Chem 289: $1345-1354$

Lee KT, Hsieh CC, Tsai WC, Tang PW, Liu IH, Chai CY, Sheu SH, and Lai WT (2012) Characteristics of atrial substrates for atrial tachyarrhythmias induced in aged and hypercholesterolemic rabbits. Pacing Clin Electrophysiol 35: $544-552$

Lee PJ and Pogwizd SM (2006) Micropatterns of propagation. Adv Cardiol 42: 86-106.

Lee TM, Lin MS, Chou TF, Tsai CH, and Chang NC (2004) Adjunctive 17betaestradiol administration reduces infarct size by altered expression of canine myocardial connexin43 protein. Cardiovasc Res 63:109-117.

Lee-Kubli CA, Ingves M, Henry KW, Shiao R, Collyer E, Tuszynski MH, and Campana WM (2016) Analysis of the behavioral, cellular and molecular characteristics of pain in severe rodent spinal cord injury. Exp Neurol 278: 91-104.

Lehnardt S, Massillon L, Follett P, Jensen FE, Ratan R, Rosenberg PA, Volpe JJ, and Vartanian T (2003) Activation of innate immunity in the CNS triggers neurodegeneration through a Toll-like receptor 4-dependent pathway. Proc Natl Acad Sci USA 100:8514-8519.

Leithe E and Rivedal E (2004) Ubiquitination and down-regulation of gap junction protein connexin-43 in response to 12 -O-tetradecanoylphorbol 13-acetate treatment. J Biol Chem 279:50089-50096.

Leithe E, Brech A, and Rivedal E (2006) Endocytic processing of connexin43 gap junctions: a morphological study. Biochem $J$ 393:59-67.

Leithe E, Kjenseth A, Sirnes S, Stenmark H, Brech A, and Rivedal E (2009) Ubiquitylation of the gap junction protein connexin- 43 signals its trafficking from early endosomes to lysosomes in a process mediated by Hrs and Tsg101. $J$ Cell Sci 122:3883-3893.

Leo-Macias A, Agullo-Pascual E, and Delmar M (2016) The cardiac connexome: noncanonical functions of connexin 43 and their role in cardiac arrhythmias. Semin Cell Dev Biol 50:13-21.

León-Paravic CG, Figueroa VA, Guzmán DJ, Valderrama CF, Vallejos AA, Fiori MC, Altenberg GA, Reuss L, and Retamal MA (2014) Carbon monoxide (CO) is a novel inhibitor of connexin hemichannels. J Biol Chem 289:36150-36157.

Lesh MD, Pring M, and Spear JF (1989) Cellular uncoupling can unmask dispersion of action potential duration in ventricular myocardium. A computer modeling study. Circ Res 65:1426-1440.

Lewandowski R, Procida K, Vaidyanathan R, Coombs W, Jalife J, Nielsen MS, Taffet SM, and Delmar M (2008) RXP-E: a connexin43-binding peptide that prevents action potential propagation block. Circ Res 103:519-526.

Leybaert L, Braet K, Vandamme W, Cabooter L, Martin PE, and Evans WH (2003) Connexin channels, connexin mimetic peptides and ATP release. Cell Commun Adhes 10:251-257.

Leykauf K, Salek M, Bomke J, Frech M, Lehmann WD, Dürst M, and Alonso A (2006) Ubiquitin protein ligase Nedd4 binds to connexin43 by a phosphorylationmodulated process. J Cell Sci 119:3634-3642.

Li C, Meng Q, Yu X, Jing X, Xu P, and Luo D (2012a) Regulatory effect of connexin 43 on basal $\mathrm{Ca}^{2+}$ signaling in rat ventricular myocytes. PLoS One 7:e36165.

Li DQ, Chen WX, Zhou YP, and Han Y (2012b) Effect of ramipril on the regulation of the expression of connexins 40 and 43 in a rabbit model of arterial balloon injury. Mol Med Rep 6:565-569. 
Li F, Sugishita K, Su Z, Ueda I, and Barry WH (2001) Activation of connexin-43 hemichannels can elevate $\left[\mathrm{Ca}^{(2+)}\right] \mathrm{i}$ and $\left[\mathrm{Na}^{(+)}\right] \mathrm{i}$ in rabbit ventricular myocytes during metabolic inhibition. J Mol Cell Cardiol 33:2145-2155.

Li G, Whittaker P, Yao M, Kloner RA, and Przyklenk K (2002) The gap junction uncoupler heptanol abrogates infarct size reduction with preconditioning in mouse hearts. Cardiovasc Pathol 11:158-165.

Li H, Spagnol G, Zheng L, Stauch KL, and Sorgen PL (2016) Regulation of Connexin43 Function and Expression by Tyrosine Kinase 2. J Biol Chem 291: $15867-15880$.

Li L, He L, Wu D, Chen L, and Jiang Z (2015) Pannexin-1 channels and their emerging functions in cardiovascular diseases. Acta Biochim Biophys Sin (Shanghai) 47:391-396.

Li X, Heinzel FR, Boengler K, Schulz R, and Heusch G (2004a) Role of connexin 43 in ischemic preconditioning does not involve intercellular communication through gap junctions. J Mol Cell Cardiol 36:161-163.

Li X, Ionescu AV, Lynn BD, Lu S, Kamasawa N, Morita M, Davidson KG, Yasumura T, Rash JE, and Nagy JI (2004b) Connexin47, connexin29 and connexin32 co-expression in oligodendrocytes and $\mathrm{Cx} 47$ association with zonula occludens-1 (ZO-1) in mouse brain. Neuroscience 126:611-630.

Li X, Olson C, Lu S, Kamasawa N, Yasumura T, Rash JE, and Nagy JI (2004c) Neuronal connexin36 association with zonula occludens-1 protein (ZO-1) in mouse brain and interaction with the first PDZ domain of ZO-1. Eur J Neurosci 19 $2132-2146$

Li X, Kamasawa N, Ciolofan C, Olson CO, Lu S, Davidson KG, Yasumura T, Shigemoto R, Rash JE, and Nagy JI (2008a) Connexin45-containing neuronal gap junctions in rodent retina also contain connexin 36 in both apposing hemiplaques, forming bihomotypic gap junctions, with scaffolding contributed by zonula occludens-1. J Neurosci 28:9769-9789.

Li X, Penes M, Odermatt B, Willecke K, and Nagy JI (2008b) Ablation of Cx47 in transgenic mice leads to the loss of MUPP1, ZONAB and multiple connexins at oligodendrocyte-astrocyte gap junctions. Eur J Neurosci 28:1503-1517.

Liao Y, Regan CP, Manabe I, Owens GK, Day KH, Damon DN, and Duling BR (2007) Smooth muscle-targeted knockout of connexin43 enhances neointimal formation in response to vascular injury. Arterioscler Thromb Vasc Biol 27:1037-1042.

Lichtenstein A, Minogue PJ, Beyer EC, and Berthoud VM (2011) Autophagy: a pathway that contributes to connexin degradation. J Cell Sci 124:910-920.

Lin D, Boyle DL, and Takemoto DJ (2003a) IGF-I-induced phosphorylation of connexin 43 by PKCgamma: regulation of gap junctions in rabbit lens epithelial cells. Invest Ophthalmol Vis Sci 44:1160-1168.

Lin D, Zhou J, Zelenka PS, and Takemoto DJ (2003b) Protein kinase Cgamma regulation of gap junction activity through caveolin-1-containing lipid rafts. Invest Ophthalmol Vis Sci 44:5259-5268.

Lin D, Lobell S, Jewell A, and Takemoto DJ (2004) Differential phosphorylation of connexin 46 and connexin 50 by $\mathrm{H} 2 \mathrm{O} 2$ activation of protein kinase Cgamma. Mol Vis 10:688-695.

Lin H, Ogawa K, Imanaga I, and Tribulova N (2006a) Alterations of connexin 43 in the diabetic rat heart. Adv Cardiol 42:243-254.

Lin H, Ogawa K, Imanaga I, and Tribulova N (2006b) Remodeling of connexin 43 in the diabetic rat heart. Mol Cell Biochem 290:69-78.

Lin J, Steenbergen C, Murphy E, and Sun J (2009) Estrogen receptor-beta activation results in S-nitrosylation of proteins involved in cardioprotection. Circulation 120: $245-254$.

Lin JH, Weigel H, Cotrina ML, Liu S, Bueno E, Hansen AJ, Hansen TW, Goldman S, and Nedergaard M (1998) Gap-junction-mediated propagation and amplification of cell injury. Nat Neurosci 1:494-500.

Lin JH, Takano T, Cotrina ML, Arcuino G, Kang J, Liu S, Gao Q, Jiang L, Li F, Lichtenberg-Frate H, et al. (2002) Connexin 43 enhances the adhesivity and mediates the invasion of malignant glioma cells. $J$ Neurosci 22:4302-4311.

Lin LC, Wu CC, Yeh HI, Lu LS, Liu YB, Lin SF, and Lee YT (2005) Downregulated myocardial connexin 43 and suppressed contractility in rabbits subjected to a cholesterol-enriched diet. Lab Invest 85:1224-1237.

Lin R, Warn-Cramer BJ, Kurata WE, and Lau AF (2001) v-Src phosphorylation of connexin 43 on Tyr247 and Tyr265 disrupts gap junctional communication. J Cell Biol 154:815-827.

Lin X, Gemel J, Glass A, Zemlin CW, Beyer EC, and Veenstra RD (2010) Connexin40 and connexin43 determine gating properties of atrial gap junction channels. $J \mathrm{Mol}$ Cell Cardiol 48:238-245.

Lin X, Xu Q, and Veenstra RD (2014) Functional formation of heterotypic gap junction channels by connexins-40 and -43. Channels (Austin) 8:433-443.

Lindsey ML, Escobar GP, Mukherjee R, Goshorn DK, Sheats NJ, Bruce JA, Mains IM, Hendrick JK, Hewett KW, Gourdie RG, et al. (2006) Matrix metalloproteinase7 affects connexin-43 levels, electrical conduction, and survival after myocardial infarction. Circulation 113:2919-2928.

Liu F, Arce FT, Ramachandran S, and Lal R (2006) Nanomechanics of hemichannel conformations: connexin flexibility underlying channel opening and closing. J Biol Chem 281:23207-23217.

Liu J, Ek Vitorin JF, Weintraub ST, Gu S, Shi Q, Burt JM, and Jiang JX (2011) Phosphorylation of connexin 50 by protein kinase A enhances gap junction and hemichannel function. $J$ Biol Chem 286:16914-16928.

Liu S, Taffet S, Stoner L, Delmar M, Vallano ML, and Jalife J (1993) A structura basis for the unequal sensitivity of the major cardiac and liver gap junctions to intracellular acidification: the carboxyl tail length. Biophys J 64:1422-1433.

Liu Y, Li H, Xia W, Yu S, Huang C, and Huang H (2014) Electrophysiological effect of rotigaptide in rabbits with heart failure. Arch Med Sci 10:374-380.

Liu Y, Fu YQ, Peng WJ, Yu YR, Wu YS, Yan H, Huang QR, He M, and Luo D (2016) Rutaecarpine reverses the altered connexin expression pattern induced by oxidized low-density lipoprotein in monocytes. $J$ Cardiovasc Pharmacol 67:519-525.

Livny O, Kaplan I, Reifen R, Polak-Charcon S, Madar Z, and Schwartz B (2002) Lycopene inhibits proliferation and enhances gap-junction communication of KB-1 human oral tumor cells. J Nutr 132:3754-3759.
Lo EH, Dalkara T, and Moskowitz MA (2003) Mechanisms, challenges and opportunities in stroke Nat Rev Neurosci 4:399-415.

Locke D, Bian S, Li H, and Harris AL (2009) Post-translational modifications of connexin26 revealed by mass spectrometry. Biochem J 424:385-398.

Locke D, Kieken F, Tao L, Sorgen PL, and Harris AL (2011) Mechanism for modulation of gating of connexin26-containing channels by taurine. J Gen Physiol 138: 321-339.

Lohman AW and Isakson BE (2014) Differentiating connexin hemichannels and pannexin channels in cellular ATP release. FEBS Lett 588:1379-1388.

Lohman AW, Billaud M, and Isakson BE (2012) Mechanisms of ATP release and signalling in the blood vessel wall. Cardiovasc Res 95:269-280.

Lohman AW, Leskov IL, Butcher JT, Johnstone SR, Stokes TA, Begandt D, DeLalio LJ, Best AK, Penuela S, Leitinger N, et al. (2015) Pannexin 1 channels regulate leukocyte emigration through the venous endothelium during acute inflammation. Nat Commun 6:7965.

Long MA, Deans MR, Paul DL, and Connors BW (2002) Rhythmicity without synchrony in the electrically uncoupled inferior olive. J Neurosci 22:10898-10905.

Lopez W, Gonzalez J, Liu Y, Harris AL, and Contreras JE (2013) Insights on the mechanisms of $\mathrm{Ca}^{(2+)}$ regulation of connexin 26 hemichannels revealed by human pathogenic mutations (D50N/Y). J Gen Physiol 142:23-35.

Lopez W, Ramachandran J, Alsamarah A, Luo Y, Harris AL, and Contreras JE (2016) Mechanism of gating by calcium in connexin hemichannels. Proc Natl Acad Sci USA 113:E7986-E7995.

Lorentz R, Shao Q, Huang T, Fishman GI, and Laird DW (2012) Characterization of gap junction proteins in the bladder of $\mathrm{Cx} 43$ mutant mouse models of oculodentodigital dysplasia. J Membr Biol 245:345-355.

Lu D, Soleymani S, Madakshire R, and Insel PA (2012a) ATP released from cardiac fibroblasts via connexin hemichannels activates profibrotic P2Y2 receptors. FASEB J 26:2580-2591.

Lu G, Haider HKh, Porollo A, and Ashraf M (2010) Mitochondria-specific transgenic overexpression of connexin-43 simulates preconditioning-induced cytoprotection of stem cells. Cardiovasc Res 88:277-286.

Lu G, Jiang S, Ashraf M, and Haider KH (2012b) Subcellular preconditioning of stem cells: mito-Cx43 gene targeting is cytoprotective via shift of mitochondrial Bak and Bcl-xL balance. Regen Med 7:323-334.

Lübkemeier I, Requardt RP, Lin X, Sasse P, Andrié R, Schrickel JW, Chkourko H, Bukauskas FF, Kim JS, Frank M, et al. (2013) Deletion of the last five C-terminal amino acid residues of connexin43 leads to lethal ventricular arrhythmias in mice without affecting coupling via gap junction channels. Basic Res Cardiol 108:348.

Lübkemeier I, Bosen F, Kim JS, Sasse P, Malan D, Fleischmann BK, and Willecke K (2015) Human connexin43E42K mutation from a sudden infant death victim leads to impaired ventricular activation and neonatal death in mice. Circ Cardiovasc Genet 8:21-29.

Lurtz MM and Louis CF (2007) Intracellular calcium regulation of connexin43. Am J Physiol Cell Physiol 293:C1806-C1813.

Maass K, Ghanem A, Kim JS, Saathoff M, Urschel S, Kirfel G, Grümmer R, Kretz M, Lewalter T, Tiemann K, et al. (2004) Defective epidermal barrier in neonatal mice lacking the C-terminal region of connexin43. Mol Biol Cell 15:4597-4608.

Maass K, Chase SE, Lin X, and Delmar M (2009) Cx43 CT domain influences infarct size and susceptibility to ventricular tachyarrhythmias in acute myocardial infarction. Cardiovasc Res 84:361-367.

MacDonald BT, Tamai K, and He X (2009) Wnt/beta-catenin signaling: components, mechanisms, and diseases. Dev Cell 17:9-26.

Machtaler S, Dang-Lawson M, Choi K, Jang C, Naus CC, and Matsuuchi L (2011) The gap junction protein $\mathrm{Cx} 43$ regulates B-lymphocyte spreading and adhesion. $J$ Cell Sci 124:2611-2621.

MacVicar BA and Thompson RJ (2010) Non-junction functions of pannexin-1 channels. Trends Neurosci 33.93-102.

Maeda S and Tsukihara T (2011) Structure of the gap junction channel and its implications for its biological functions. Cell Mol Life Sci 68:1115-1129.

Maeda S, Nakagawa S, Suga M, Yamashita E, Oshima A, Fujiyoshi Y, and Tsukihara $\mathrm{T}$ (2009) Structure of the connexin 26 gap junction channel at 3.5 A resolution. Nature 458:597-602

Maezawa I and Jin LW (2010) Rett syndrome microglia damage dendrites and synapses by the elevated release of glutamate. $J$ Neurosci 30:5346-5356.

Makita N, Seki A, Sumitomo N, Chkourko H, Fukuhara S, Watanabe H, Shimizu W, Bezzina CR, Hasdemir C, Mugishima H, et al. (2012) A connexin40 mutation associated with a malignant variant of progressive familial heart block type I. Circ Arrhythm Electrophysiol 5:163-172.

Mallard C, Davidson JO, Tan S, Green CR, Bennet L, Robertson NJ, and Gunn AJ (2014) Astrocytes and microglia in acute cerebral injury underlying cerebral palsy associated with preterm birth. Pediatr Res 75:234-240.

Mandapati R, Asano Y, Baxter WT, Gray R, Davidenko J, and Jalife J (1998) Quantification of effects of global ischemia on dynamics of ventricular fibrillation in isolated rabbit heart. Circulation 98:1688-1696.

Manjunath CK and Page E (1985) Cell biology and protein composition of cardiac gap junctions. Am J Physiol 248:H783-H791.

Manjunath CK, Nicholson BJ, Teplow D, Hood L, Page E, and Revel JP (1987) The cardiac gap junction protein (Mr 47,000) has a tissue-specific cytoplasmic domain of Mr 17,000 at its carboxy-terminus. Biochem Biophys Res Commun 142:228-234.

Mao Y, Tonkin RS, Nguyen T, O'Carroll SJ, Nicholson LF, Green CR, Moalem-Taylor G, and Gorrie CA (2016) Systemic administration of connexin43 mimetic peptide improves functional recovery after traumatic spinal cord injury in adult rats. $J$ Neurotrauma 34:707-719.

Marandykina A, Palacios-Prado N, Rimkute L, Skeberdis VA, and Bukauskas FF (2013) Regulation of connexin36 gap junction channels by $n$-alkanols and arachidonic acid. J Physiol 591:2087-2101.

Marc RE, Jones BW, Watt CB, Vazquez-Chona F, Vaughan DK, and Organisciak DT (2008) Extreme retinal remodeling triggered by light damage: implications for age related macular degeneration. Mol Vis 14:782-806. 
Marchesi VT (2016) Gain-of-function somatic mutations contribute to inflammation and blood vessel damage that lead to Alzheimer dementia: a hypothesis. FASEB $J$ 30:503-506.

Martin PE, Steggles J, Wilson C, Ahmad S, and Evans WH (2000) Targeting motifs and functional parameters governing the assembly of connexins into gap junctions. Biochem J 349:281-287.

Martin PE, Blundell G, Ahmad S, Errington RJ, and Evans WH (2001) Multiple pathways in the trafficking and assembly of connexin 26,32 and 43 into gap junction intercellular communication channels. J Cell Sci 114:3845-3855.

Martin PE, Wall C, and Griffith TM (2005) Effects of connexin-mimetic peptides on gap junction functionality and connexin expression in cultured vascular cells. $\mathrm{Br} J$ Pharmacol 144:617-627.

Martins-Marques T, Anjo SI, Pereira P, Manadas B, and Girão H (2015a) Interacting network of the Gap Junction (GJ) protein Connexin43 (Cx43) is modulated by ischemia and reperfusion in the heart. Mol Cell Proteomics 14: 3040-3055.

Martins-Marques T, Catarino S, Marques C, Matafome P, Ribeiro-Rodrigues T, Baptista R, Pereira P, and Girão H (2015b) Heart ischemia results in connexin43 ubiquitination localized at the intercalated discs. Biochimie 112:196-201.

Martins-Marques T, Catarino S, Zuzarte M, Marques C, Matafome P, Pereira P, and Girão H (2015c) Ischaemia-induced autophagy leads to degradation of gap junction protein connexin43 in cardiomyocytes. Biochem $J$ 467:231-245.

Massey KD, Minnich BN, and Burt JM (1992) Arachidonic acid and lipoxygenase metabolites uncouple neonatal rat cardiac myocyte pairs. Am J Physiol 263: C494-C501.

Matchkov VV, Rahman A, Bakker LM, Griffith TM, Nilsson H, and Aalkjaer C (2006) Analysis of effects of connexin-mimetic peptides in rat mesenteric small arteries. Am J Physiol Heart Circ Physiol 291:H357-H367.

Matsushita T, Oyamada M, Fujimoto K, Yasuda Y, Masuda S, Wada Y, Oka T, and Takamatsu $\mathrm{T}$ (1999) Remodeling of cell-cell and cell-extracellular matrix interactions at the border zone of rat myocardial infarcts. Circ Res 85:1046-1055.

Matsuuchi L and Naus CC (2013) Gap junction proteins on the move: connexins, the cytoskeleton and migration. Biochim Biophys Acta 1828:94-108.

Matsuyama D and Kawahara K (2011) Oxidative stress-induced formation of a positive-feedback loop for the sustained activation of p38 MAPK leading to the loss of cell division in cardiomyocytes soon after birth. Basic Res Cardiol 106:815-828.

Maurer P and Weingart R (1987) Cell pairs isolated from adult guinea pig and rat hearts: effects of $\left[\mathrm{Ca}^{2+}\right] \mathrm{i}$ on nexal membrane resistance. Pflugers Arch 409 394-402.

May D, Tress O, Seifert G, and Willecke K (2013) Connexin47 protein phosphorylation and stability in oligodendrocytes depend on expression of connexin 43 protein in astrocytes. J Neurosci 33:7985-7996.

McArthur L, Chilton L, Smith GL, and Nicklin SA (2015) Electrical consequences of cardiac myocyte: fibroblast coupling. Biochem Soc Trans 43:513-518.

McLachlan E, Manias JL, Gong XQ, Lounsbury CS, Shao Q, Bernier SM, Bai D, and Laird DW (2005) Functional characterization of oculodentodigital dysplasiaassociated Cx43 mutants. Cell Commun Adhes 12:279-292.

McNutt NS and Weinstein RS (1970) The ultrastructure of the nexus. A correlated thin-section and freeze-cleave study. $J$ Cell Biol 47:666-688.

Meens MJ, Pfenniger A, and Kwak BR (2012) Risky communication in atherosclerosis and thrombus formation. Swiss Med Wkly 142:w13553.

Mei X, Ezan P, Giaume C, and Koulakoff A (2010) Astroglial connexin immunoreactivity is specifically altered at $\beta$-amyloid plaques in $\beta$-amyloid precursor protein presenilin1 mice. Neuroscience 171:92-105.

Menichella DM, Goodenough DA, Sirkowski E, Scherer SS, and Paul DL (2003) Connexins are critical for normal myelination in the CNS. $J$ Neurosci 23 5963-5973.

Menichella DM, Majdan M, Awatramani R, Goodenough DA, Sirkowski E, Schere SS, and Paul DL (2006) Genetic and physiological evidence that oligodendrocyte gap junctions contribute to spatial buffering of potassium released during neuronal activity. $J$ Neurosci 26:10984-10991.

Menko AS and Boettiger D (1988) Inhibition of chicken embryo lens differentiation and lens junction formation in culture by pp60v-src. Mol Cell Biol 8:1414-1420.

Meşe G, Richard G, and White TW (2007) Gap junctions: basic structure and function. J Invest Dermatol 127:2516-2524.

Meunier C, Wang N, Yi C, Dallerac G, Ezan P, Koulakoff A, Leybaert L, and Giaume C (2017) Contribution of astroglial Cx43 hemichannels to the modulation of glutamatergic currents by $\mathrm{D}$-serine in the mouse prefrontal cortex. $J$ Neurosci DOI: 10.1523/JNEUROSCI.2204-16.2017 [published ahead of print]

Michela P, Velia V, Aldo P, and Ada P (2015) Role of connexin 43 in cardiovascular diseases. Eur $J$ Pharmacol 768:71-76.

Miners S, Moulding H, de Silva R, and Love S (2014) Reduced vascular endothelial growth factor and capillary density in the occipital cortex in dementia with Lewy bodies. Brain Pathol 24:334-343.

Miro-Casas E, Ruiz-Meana M, Agullo E, Stahlhofen S, Rodríguez-Sinovas A, Cabestrero A, Jorge I, Torre I, Vazquez J, Boengler K, et al. (2009) Connexin43 in cardiomyocyte mitochondria contributes to mitochondrial potassium uptake. Cardiovasc Res 83:747-756.

Mitra SS, Xu J, and Nicholson BJ (2012) Coregulation of multiple signaling mechanisms in pp60v-Src-induced closure of $\mathrm{Cx} 43$ gap junction channels. J Membr Biol 245:495-506.

Miura T, Miki T, and Yano T (2010) Role of the gap junction in ischemic preconditioning in the heart. Am J Physiol Heart Circ Physiol 298:H1115-H1125.

Mlinar B and Enyeart JJ (1993) Block of current through T-type calcium channels by trivalent metal cations and nickel in neural rat and human cells. $J$ Physiol $\mathbf{4 6 9}$ $639-652$

Mograbi B, Corcelle E, Defamie N, Samson M, Nebout M, Segretain D, Fénichel P, and Pointis G (2003) Aberrant Connexin 43 endocytosis by the carcinogen lindane involves activation of the ERK/mitogen-activated protein kinase pathway. Carcinogenesis 24:1415-1423.
Molica F, Meens MJ, Morel S, and Kwak BR (2014) Mutations in cardiovascular connexin genes. Biol Cell 106:269-293.

Molica F, Morel S, Meens MJ, Denis JF, Bradfield PF, Penuela S, Zufferey A, Monyer H, Imhof BA, Chanson M, et al. (2015) Functional role of a polymorphism in the Pannexin1 gene in collagen-induced platelet aggregation. Thromb Haemost 114 325-336.

Molica F, Stierlin FB, Fontana P, and Kwak BR (2017) Pannexin- and connexinmediated intercellular communication in platelet function. Int J Mol Sci 18:85.

Monder C, Stewart PM, Lakshmi V, Valentino R, Burt D, and Edwards CR (1989) Licorice inhibits corticosteroid 11 beta-dehydrogenase of rat kidney and liver: in vivo and in vitro studies. Endocrinology 125:1046-1053.

Montero TD and Orellana JA (2015) Hemichannels: new pathways for gliotransmitter release. Neuroscience 286:45-59.

Moore KB and O'Brien $\mathrm{J}$ (2015) Connexins in neurons and glia: targets for intervention in disease and injury. Neural Regen Res 10:1013-1017.

Moreau A, Gosselin-Badaroudine P, and Chahine M (2014) Biophysics, pathophysiology, and pharmacology of ion channel gating pores. Front Pharmacol 5:53.

Morel S, Burnier L, Roatti A, Chassot A, Roth I, Sutter E, Galan K, Pfenniger A, Chanson M, and Kwak BR (2010) Unexpected role for the human Cx37 C1019T polymorphism in tumour cell proliferation. Carcinogenesis 31:1922-1931.

Morel S, Braunersreuther V, Chanson M, Bouis D, Rochemont V, Foglia B, Pelli G, Sutter E, Pinsky DJ, Mach F, et al. (2014a) Endothelial Cx40 limits myocardial ischaemia/reperfusion injury in mice. Cardiovasc Res 102:329-337.

Morel S, Chanson M, Nguyen TD, Glass AM, Richani Sarieddine MZ, Meens MJ, Burnier L, Kwak BR, and Taffet SM (2014b) Titration of the gap junction protein Connexin43 reduces atherogenesis. Thromb Haemost 112:390-401.

Morel S, Christoffersen C, Axelsen LN, Montecucco F, Rochemont V, Frias MA, Mach F, James RW, Naus CC, Chanson M, et al. (2016) Sphingosine-1-phosphate reduces ischaemia-reperfusion injury by phosphorylating the gap junction protein connexin43. Cardiovasc Res 109:385-396.

Moreno AP and Lau AF (2007) Gap junction channel gating modulated through protein phosphorylation. Prog Biophys Mol Biol 94:107-119.

Moreno AP, Sáez JC, Fishman GI, and Spray DC (1994) Human connexin43 gap junction channels. Regulation of unitary conductances by phosphorylation. Circ Res 74:1050-1057.

Moreno AP, Chanson M, Elenes S, Anumonwo J, Scerri I, Gu H, Taffet SM, and Delmar M (2002) Role of the carboxyl terminal of connexin43 in transjunctional fast voltage gating. Circ Res 90:450-457.

Morley GE, Taffet SM, and Delmar M (1996) Intramolecular interactions mediate pH regulation of connexin43 channels. Biophys J 70:1294-1302.

Morley GE, Ek-Vitorín JF, Taffet SM, and Delmar M (1997) Structure of connexin43 and its regulation by pHi. J Cardiovasc Electrophysiol 8:939-951.

Morley GE, Vaidya D, Samie FH, Lo C, Delmar M, and Jalife J (1999) Characterization of conduction in the ventricles of normal and heterozygous $\mathrm{Cx} 43$ knockout mice using optical mapping. $J$ Cardiovasc Electrophysiol 10:1361-1375.

Morley GE, Vaidya D, and Jalife J (2000) Characterization of conduction in the entricles of normal and heterozygous $\mathrm{Cx} 43$ knockout mice using optical mapping. $J$ Cardiovasc Electrophysiol 11:375-377.

Mühlfeld C, Cetegen C, Freese S, Volkmann R, Hellige G, and Vetterlein F (2010) Phosphorylation of extrajunctional $\mathrm{Cx} 43$ in ischemic-preconditioned rat hearts. $J$ Surg Res 162:e1-e8.

Müller A and Dhein S (1993) Sodium channel blockade enhances dispersion of the cardiac action potential duration. A computer simulation study. Basic Res Cardiol 88:11-22.

Müller A, Gottwald M, Tudyka T, Linke W, Klaus W, and Dhein S (1997a) Increase in gap junction conductance by an antiarrhythmic peptide. Eur $J$ Pharmacol 327 $65-72$

Müller A, Schaefer T, Linke W, Tudyka T, Gottwald M, Klaus W, and Dhein S (1997b) Actions of the antiarrhythmic peptide AAP10 on intercellular coupling. Naunyn Schmiedebergs Arch Pharmacol 356:76-82.

Müller DJ, Hand GM, Engel A, and Sosinsky GE (2002) Conformational changes in surface structures of isolated connexin 26 gap junctions. $E M B O J \mathbf{2 1}$ 3598-3607.

Mulligan SJ and MacVicar BA (2004) Calcium transients in astrocyte endfeet cause cerebrovascular constrictions. Nature 431:195-199.

Murray SA, Nickel BM, and Gay VL (2004) Endocytosis of connexin protein in adrenal cells. Endocr Res 30:647-654.

Musil LS and Goodenough DA (1993) Multisubunit assembly of an integral plasma membrane channel protein, gap junction connexin43, occurs after exit from the ER. Cell 74:1065-1077.

Musil LS and Goodenough DA (1995) Biochemical analysis of connexon assembly, in Intercellular communication through gap junctions (Kanno Y, Kataoka K, Shiba Y, Shibata Y, and Shimazu T eds) Elsevier Science Publ., Amsterdam.

Musil LS, Beyer EC, and Goodenough DA (1990) Expression of the gap junction protein connexin43 in embryonic chick lens: molecular cloning, ultrastructural localization, and post-translational phosphorylation. J Membr Biol 116:163-175.

Nagy JI, Li W, Hertzberg EL, and Marotta CA (1996) Elevated connexin43 immunoreactivity at sites of amyloid plaques in Alzheimer's disease. Brain Res $\mathbf{7 1 7}$ $173-178$

Nagy JI, Patel D, Ochalski PA, and Stelmack GL (1999) Connexin30 in rodent, cat and human brain: selective expression in gray matter astrocytes, co-localization with connexin 43 at gap junctions and late developmental appearance. Neuroscience 88:447-468.

Nagy JI, Li X, Rempel J, Stelmack G, Patel D, Staines WA, Yasumura T, and Rash JE (2001) Connexin26 in adult rodent central nervous system: demonstration at astrocytic gap junctions and colocalization with connexin 30 and connexin $43 . J$ Comp Neurol 441:302-323.

Nakase T, Fushiki S, Söhl G, Theis M, Willecke K, and Naus CC (2003) Neuroprotective role of astrocytic gap junctions in ischemic stroke. Cell Commun Adhes 10:413-417. 
Nakase T, Söhl G, Theis M, Willecke K, and Naus CC (2004) Increased apoptosis and inflammation after focal brain ischemia in mice lacking connexin43 in astrocytes. Am J Pathol 164:2067-2075.

Nakase T, Yoshida Y, and Nagata K (2006) Enhanced connexin 43 immunoreactivity in penumbral areas in the human brain following ischemia. Glia 54:369-375.

Nakase T, Maeda T, Yoshida Y, and Nagata K (2009) Ischemia alters the expression of connexins in the aged human brain. J Biomed Biotechnol 2009:147946.

Nao T, Ohkusa T, Hisamatsu Y, Inoue N, Matsumoto T, Yamada J, Shimizu A, Yoshiga Y, Yamagata T, Kobayashi S, et al. (2003) Comparison of expression of connexin in right atrial myocardium in patients with chronic atrial fibrillation versus those in sinus rhythm. Am J Cardiol 91:678-683.

Naus CC and Giaume C (2016) Bridging the gap to therapeutic strategies based on connexin/pannexin biology. J Transl Med 14:330.

Naus CC, Ozog MA, Bechberger JF, and Nakase T (2001) A neuroprotective role for gap junctions. Cell Commun Adhes 8:325-328.

Naus CC, Aftab Q, and Sin WC (2016) Common mechanisms linking connexin43 to neural progenitor cell migration and glioma invasion. Semin Cell Dev Biol 50 59-66.

Neary JT, Kang Y, Willoughby KA, and Ellis EF (2003) Activation of extracellular signal-regulated kinase by stretch-induced injury in astrocytes involves extracellular ATP and P2 purinergic receptors. J Neurosci 23:2348-2356.

Neijssen J, Pang B, and Neefjes J (2007) Gap junction-mediated intercellular communication in the immune system. Prog Biophys Mol Biol 94:207-218.

Nguyen TP, Qu Z, and Weiss JN (2014) Cardiac fibrosis and arrhythmogenesis: the road to repair is paved with perils. $J$ Mol Cell Cardiol 70:83-91.

Ni M, Ruan L, and Zhang C (2015) Antiarrhythmic peptide AAP10 prevents arrhythmias induced by protein kinase $\mathrm{C}$ activation in rabbit left ventricular wedges. Int Heart $J$ 56:234-238.

Nickel B, Boller M, Schneider K, Shakespeare T, Gay V, and Murray SA (2013) Visualizing the effect of dynamin inhibition on annular gap vesicle formation and fission. J Cell Sci 126:2607-2616.

Nielsen PA and Kumar NM (2003) Differences in expression patterns between mouse connexin-30.2 (Cx30.2) and its putative human orthologue, connexin-31.9. FEBS Lett 540:151-156.

Nielsen PA, Beahm DL, Giepmans BNG, Baruch A, Hall JE, and Kumar NM (2002) Molecular cloning, functional expression, and tissue distribution of a novel human gap junction-forming protein, connexin-31.9. Interaction with zona occludens protein-1. J Biol Chem 277:38272-38283.

Nodin C, Nilsson M, and Blomstrand F (2005) Gap junction blockage limits intercellular spreading of astrocytic apoptosis induced by metabolic depression. $J$ Neurochem 94:1111-1123.

Noma A and Tsuboi N (1987) Dependence of junctional conductance on proton, calcium and magnesium ions in cardiac paired cells of guinea-pig. J Physiol 382 193-211.

Nygren A, Olson ML, Chen KY, Emmett T, Kargacin G, and Shimoni Y (2007) Propagation of the cardiac impulse in the diabetic rat heart: reduced conduction reserve. J Physiol 580:543-560.

O'Carroll SJ, Alkadhi M, Nicholson LF, and Green CR (2008) Connexin 43 mimetic peptides reduce swelling, astrogliosis, and neuronal cell death after spinal cord injury. Cell Commun Adhes 15:27-42.

O'Carroll SJ, Gorrie CA, Velamoor S, Green CR, and Nicholson LF (2013) Connexin43 mimetic peptide is neuroprotective and improves function following spinal cord injury. Neurosci Res 75:256-267.

O'Quinn MP, Palatinus JA, Harris BS, Hewett KW, and Gourdie RG (2011) A peptide mimetic of the connexin 43 carboxyl terminus reduces gap junction remodeling and induced arrhythmia following ventricular injury. Circ Res 108:704-715.

Oh S and Bargiello TA (2015) Voltage regulation of connexin channel conductance. Yonsei Med J 56:1-15.

Oh S, Abrams CK, Verselis VK, and Bargiello TA (2000) Stoichiometry of transjunctional voltage-gating polarity reversal by a negative charge substitution in the amino terminus of a connexin32 chimera. $J$ Gen Physiol 116:13-31.

Oishi S, Sasano T, Tateishi Y, Tamura N, Isobe M, and Furukawa T (2012) Stretch of atrial myocytes stimulates recruitment of macrophages via ATP released through gap-junction channels. J Pharmacol Sci 120:296-304.

Olsen KB, Axelsen LN, Braunstein TH, Sørensen CM, Andersen CB, Ploug T, Holstein-Rathlou NH, and Nielsen MS (2013) Myocardial impulse propagation is impaired in right ventricular tissue of Zucker diabetic fatty (ZDF) rats. Cardiovasc Diabetol 12:19.

Omasits U, Ahrens CH, Müller S, and Wollscheid B (2014) Protter: interactive protein feature visualization and integration with experimental proteomic data. Bioinformatics 30:884-886.

Ong SB, Dongworth RK, Cabrera-Fuentes HA, and Hausenloy DJ (2015) Role of the MPTP in conditioning the heart - translatability and mechanism. Br J Pharmacol 172:2074-2084

Ongstad E and Kohl P (2016) Fibroblast-myocyte coupling in the heart: Potential relevance for therapeutic interventions. J Mol Cell Cardiol 91:238-246.

Opthof T, Misier AR, Coronel R, Vermeulen JT, Verberne HJ, Frank RG, Moulijn AC, van Capelle FJ, and Janse MJ (1991) Dispersion of refractoriness in canine ventricular myocardium. Effects of sympathetic stimulation. Circ Res 68 : 1204-1215.

Orellana JA (2016) Physiological functions of glial cell hemichannels. Adv Exp Med Biol 949:93-108.

Orellana JA, Sáez PJ, Shoji KF, Schalper KA, Palacios-Prado N, Velarde V, Giaume C, Bennett MV, and Sáez JC (2009) Modulation of brain hemichannels and gap junction channels by pro-inflammatory agents and their possible role in neurodegeneration. Antioxid Redox Signal 11:369-399.

Orellana JA, Hernández DE, Ezan P, Velarde V, Bennett MV, Giaume C, and Sáez JC (2010) Hypoxia in high glucose followed by reoxygenation in normal glucose reduces the viability of cortical astrocytes through increased permeability of connexin 43 hemichannels. Glia 58:329-343.
Orellana JA, Froger N, Ezan P, Jiang JX, Bennett MV, Naus CC, Giaume C, and Sáez JC (2011a) ATP and glutamate released via astroglial connexin 43 hemichannels mediate neuronal death through activation of pannexin 1 hemichannels. $J$ Neurochem 118:826-840.

Orellana JA, Shoji KF, Abudara V, Ezan P, Amigou E, Sáez PJ, Jiang JX, Naus CC, Sáez JC, and Giaume C (2011b) Amyloid $\beta$-induced death in neurons involves glial and neuronal hemichannels. $J$ Neurosci 31:4962-4977.

Orellana JA, von Bernhardi R, Giaume C, and Sáez JC (2012) Glial hemichannels and their involvement in aging and neurodegenerative diseases. Rev Neurosci 23: 163-177.

Orellana JA, Avendaño BC, and Montero TD (2014) Role of connexins and pannexins in ischemic stroke. Curr Med Chem 21:2165-2182.

Ormonde S, Chou CY, Goold L, Petsoglou C, Al-Taie R, Sherwin T, McGhee CN, and Green CR (2012) Regulation of connexin43 gap junction protein triggers vascular recovery and healing in human ocular persistent epithelial defect wounds. $J$ Membr Biol 245:381-388.

Osbourne A, Calway T, Broman M, McSharry S, Earley J, and Kim GH (2014) Downregulation of connexin43 by microRNA-130a in cardiomyocytes results in cardiac arrhythmias. J Mol Cell Cardiol 74:53-63.

Oshima A (2014) Structure and closure of connexin gap junction channels. FEBS Let 588:1230-1237.

Oviedo-Orta E and Howard Evans W (2004) Gap junctions and connexin-mediated communication in the immune system. Biochim Biophys Acta 1662:102-112.

Oxford EM, Musa H, Maass K, Coombs W, Taffet SM, and Delmar M (2007) Connexin43 remodeling caused by inhibition of plakophilin-2 expression in cardiac cells. Circ Res 101:703-711.

Palacios-Prado N and Bukauskas FF (2012) Modulation of metabolic communication through gap junction channels by transjunctional voltage; synergistic and antagonistic effects of gating and ionophoresis. Biochim Biophys Acta 1818:1884-1894.

Palacios-Prado N, Sonntag S, Skeberdis VA, Willecke K, and Bukauskas FF (2009) Gating, permselectivity and $\mathrm{pH}$-dependent modulation of channels formed by connexin57, a major connexin of horizontal cells in the mouse retina. J Physiol 587: $3251-3269$

Palatinus JA and Gourdie RG (2016) Diabetes Increases Cryoinjury Size with Associated Effects on Cx43 Gap Junction Function and Phosphorylation in the Mouse Heart. J Diabetes Res 2016:8789617.

Palatinus JA, Rhett JM, and Gourdie RG (2012) The connexin43 carboxyl terminus and cardiac gap junction organization. Biochim Biophys Acta 1818:1831-1843.

Pannasch U, Vargová L, Reingruber J, Ezan P, Holcman D, Giaume C, Syková E, and Rouach N (2011) Astroglial networks scale synaptic activity and plasticity. Proc Natl Acad Sci USA 108:8467-8472.

Pannasch U, Freche D, Dallérac G, Ghézali G, Escartin C, Ezan P, Cohen-Salmon M, Benchenane K, Abudara V, Dufour A, et al. (2014) Connexin 30 sets synaptic strength by controlling astroglial synapse invasion. Nat Neurosci 17:549-558.

Park DJ, Wallick CJ, Martyn KD, Lau AF, Jin C, and Warn-Cramer BJ (2007) Akt phosphorylates Connexin43 on Ser373, a "mode-1" binding site for 14-3-3. Cell Commun Adhes 14:211-226.

Parthasarathi K, Ichimura H, Monma E, Lindert J, Quadri S, Issekutz A, and Bhattacharya J (2006) Connexin 43 mediates spread of $\mathrm{Ca}^{2+}$-dependent proinflammatory responses in lung capillaries. J Clin Invest 116:2193-2200.

Patel D, Gemel J, Xu Q, Simon AR, Lin X, Matiukas A, Beyer EC, and Veenstra RD (2014) Atrial fibrillation-associated connexin40 mutants make hemichannels and synergistically form gap junction channels with novel properties. FEBS Lett 588: 1458-1464.

Paul DL, Ebihara L, Takemoto LJ, Swenson KI, and Goodenough DA (1991) Connexin46, a novel lens gap junction protein, induces voltage-gated currents in nonjunctional plasma membrane of Xenopus oocytes. J Cell Biol 115:1077-1089.

Paulauskas N, Pranevicius H, Mockus J, and Bukauskas FF (2012) Stochastic 16 -state model of voltage gating of gap-junction channels enclosing fast and slow gates. Biophys J 102:2471-2480.

Paulson AF, Lampe PD, Meyer RA, TenBroek E, Atkinson MM, Walseth TF, and Johnson RG (2000) Cyclic AMP and LDL trigger a rapid enhancement in gap junction assembly through a stimulation of connexin trafficking. J Cell Sci 113: 3037-3049

Paznekas WA, Boyadjiev SA, Shapiro RE, Daniels O, Wollnik B, Keegan CE, Innis JW, Dinulos MB, Christian C, Hannibal MC, et al. (2003) Connexin 43 (GJA1) mutations cause the pleiotropic phenotype of oculodentodigital dysplasia. Am $J$ Hum Genet 72:408-418.

Paznekas WA, Karczeski B, Vermeer S, Lowry RB, Delatycki M, Laurence F, Koivisto PA, Van Maldergem L, Boyadjiev SA, Bodurtha JN, et al. (2009) GJA1 mutations, variants, and connexin 43 dysfunction as it relates to the oculodentodigital dysplasia phenotype. Hum Mutat 30:724-733.

Pecoraro M, Sorrentino R, Franceschelli S, Del Pizzo M, Pinto A, and Popolo A (2015) Doxorubicin-mediated cardiotoxicity: role of mitochondrial connexin 43 Cardiovasc Toxicol 15:366-376.

Pedersen CM, Venkatasubramanian S, Vase H, Hyldebrandt JA, Contractor H, Schmidt MR, Bøtker HE, Cruden NL, Newby DE, Kharbanda RK, et al. (2016 Rotigaptide protects the myocardium and arterial vasculature from ischaemia reperfusion injury. Br J Clin Pharmacol 81:1037-1045.

Pelegrin P and Surprenant A (2006) Pannexin-1 mediates large pore formation and interleukin-1beta release by the ATP-gated P2X7 receptor. EMBO J 25:5071-5082. Pellegrini-Giampietro DE, Gorter JA, Bennett MVL, and Zukin RS (1997) The GluR2 (GluR-B) hypothesis: $\mathrm{Ca}^{(2+)}$-permeable AMPA receptors in neurological disorders. Trends Neurosci 20:464-470.

Pelletier RM, Akpovi CD, Chen L, Kumar NM, and Vitale ML (2015) Complementary expression and phosphorylation of $\mathrm{Cx} 46$ and $\mathrm{Cx} 50$ during development and following gene deletion in mouse and in normal and orchitic mink testes. Am $J$ Physiol Regul Integr Comp Physiol 309:R255-R276.

Penes MC, Li X, and Nagy JI (2005) Expression of zonula occludens-1 (ZO-1) and the transcription factor ZO-1-associated nucleic acid-binding protein (ZONAB)-MsY3 
in glial cells and colocalization at oligodendrocyte and astrocyte gap junctions in mouse brain. Eur $J$ Neurosci 22:404-418.

Peng W, Cotrina ML, Han X, Yu H, Bekar L, Blum L, Takano T, Tian GF, Goldman SA, and Nedergaard M (2009) Systemic administration of an antagonist of the ATP-sensitive receptor P2X7 improves recovery after spinal cord injury. Proc Natl Acad Sci USA 106:12489-12493.

Penna C, Perrelli MG, Raimondo S, Tullio F, Merlino A, Moro F, Geuna S, Mancardi $\mathrm{D}$, and Pagliaro P (2009) Postconditioning induces an anti-apoptotic effect and preserves mitochondrial integrity in isolated rat hearts. Biochim Biophys Acta 1787:794-801.

Peracchia C (1987) Calmodulin-like proteins and communicating junctions. Electrical uncoupling of crayfish septate axons is inhibited by the calmodulin inhibitor W7 and is not affected by cyclic nucleotides. Pflugers Arch 408:379-385.

Peracchia C (2004) Chemical gating of gap junction channels; roles of calcium, $\mathrm{pH}$ and calmodulin. Biochim Biophys Acta 1662:61-80.

Peracchia C, Wang XG, and Peracchia LL (2000) Chemical gating of gap junction channels. Methods 20:188-195

Perez Velazquez JL, Kokarovtseva L, Sarbaziha R, Jeyapalan Z, and Leshchenko Y (2006) Role of gap junctional coupling in astrocytic networks in the determination of global ischaemia-induced oxidative stress and hippocampal damage. Eur $J$ Neurosci 23:1-10.

Perkins GA, Goodenough DA, and Sosinsky GE (1998) Formation of the gap junction intercellular channel requires a 30 degree rotation for interdigitating two apposing connexons. J Mol Biol 277:171-177.

Personius KE, Chang Q, Mentis GZ, O'Donovan MJ, and Balice-Gordon RJ (2007) Reduced gap junctional coupling leads to uncorrelated motor neuron firing and precocious neuromuscular synapse elimination. Proc Natl Acad Sci USA 104: 11808-11813.

Peters NS (1995) Myocardial gap junction organization in ischemia and infarction. Microsc Res Tech 31:375-386.

Peters NS, Coromilas J, Severs NJ, and Wit AL (1997) Disturbed connexin43 gap junction distribution correlates with the location of reentrant circuits in the epicardial border zone of healing canine infarcts that cause ventricular tachycardia Circulation 95:988-996.

Petric S, Klein S, Dannenberg L, Lahres T, Clasen L, Schmidt KG, Ding Z and Donner BC (2016) Pannexin-1 deficient mice have an increased susceptibility for strial fibrillation and show a QT-prolongation phenotype. Cell Physiol Biochem 38:487-501.

Pfahnl A and Dahl G (1999) Gating of cx46 gap junction hemichannels by calcium and voltage. Pflugers Arch 437:345-353.

Pfenniger A, Chanson M, and Kwak BR (2013) Connexins in atherosclerosis. Biochim Biophys Acta 1828:157-166.

Pfenniger A, Derouette JP, Verma V, Lin X, Foglia B, Coombs W, Roth I, Satta N, Dunoyer-Geindre S, Sorgen P, et al. (2010) Gap junction protein Cx37 interacts with endothelial nitric oxide synthase in endothelial cells. Arterioscler Thromb Vasc Biol 30:827-834.

Pfenniger A, Wohlwend A, and Kwak BR (2011) Mutations in connexin genes and disease. Eur J Clin Invest 41:103-116.

Pfenniger A, van der Laan SW, Foglia B, Dunoyer-Geindre S, Haefliger JA, Winnik S, Mach F, Pasterkamp G, James RW, and Kwak BR (2012a) Lack of association between connexin 40 polymorphisms and coronary artery disease. Atherosclerosis 222:148-153.

Pfenniger A, Wong C, Sutter E, Cuhlmann S, Dunoyer-Geindre S, Mach F, Horrevoets AJ, Evans PC, Krams R, and Kwak BR (2012b) Shear stress modulates the expression of the atheroprotective protein Cx37 in endothelial cells. J Mol Cell Cardiol 53:299-309.

Pfenniger A, Meens MJ, Pedrigi RM, Foglia B, Sutter E, Pelli G, Rochemont V, Petrova TV, Krams R, and Kwak BR (2015) Shear stress-induced atherosclerotic plaque composition in ApoE(-/-) mice is modulated by connexin37. Atherosclerosis 243:1-10.

Piehl M, Lehmann C, Gumpert A, Denizot JP, Segretain D, and Falk MM (2007) Internalization of large double-membrane intercellular vesicles by a clathrindependent endocytic process. Mol Biol Cell 18:337-347.

Pimentel RC, Yamada KA, Kléber AG, and Saffitz JE (2002) Autocrine regulation of myocyte Cx43 expression by VEGF. Circ Res 90:671-677.

Pizzuti A, Flex E, Mingarelli R, Salpietro C, Zelante L, and Dallapiccola B (2004) A homozygous GJA1 gene mutation causes a Hallermann-Streiff/ODDD spectrum phenotype. Hum Mutat 23:286.

Plenz G, Ko YS, Yeh HI, Eschert H, Sindermann JR, Dorszewski A, Hofnagel O, Robenek H, Breithardt G, and Severs NJ (2004) Upregulation of connexin43 gap junctions between neointimal smooth muscle cells. Eur J Cell Biol 83:521-530.

Pogoda K, Kameritsch P, Retamal MA, and Vega JL (2016) Regulation of gap junction channels and hemichannels by phosphorylation and redox changes: a revision. BMC Cell Biol 17 (Suppl 1);11.

Polacek D, Lal R, Volin MV, and Davies PF (1993) Gap junctional communication between vascular cells. Induction of connexin43 messenger RNA in macrophage foam cells of atherosclerotic lesions. Am J Pathol 142:593-606.

Polacek D, Bech F, McKinsey JF, and Davies PF (1997) Connexin43 gene expression in the rabbit arterial wall: effects of hypercholesterolemia, balloon injury and their combination. $J$ Vasc Res 34:19-30.

Polontchouk L, Haefliger JA, Ebelt B, Schaefer T, Stuhlmann D, Mehlhorn U, KuhnRegnier F, De Vivie ER, and Dhein S (2001) Effects of chronic atrial fibrillation on gap junction distribution in human and rat atria. J Am Coll Cardiol 38:883-891.

Polontchouk L, Ebelt B, Jackels M, and Dhein S (2002) Chronic effects of endothelin 1 and angiotensin II on gap junctions and intercellular communication in cardiac cells. FASEB J 16:87-89.

Ponsaerts R, D'hondt C, Bultynck G, Srinivas SP, Vereecke J, and Himpens B (2008) The myosin II ATPase inhibitor blebbistatin prevents thrombin-induced inhibition of intercellular calcium wave propagation in corneal endothelial cells. Invest Ophthalmol Vis Sci 49:4816-4827.
Ponsaerts R, De Vuyst E, Retamal M, D'hondt C, Vermeire D, Wang N, De Smedt H, Zimmermann P, Himpens B, Vereecke J, et al (2010) Intramolecular loop/tail interactions are essential for connexin 43-hemichannel activity. FASEB $J \mathbf{2 4}$ $4378-4395$

Ponsaerts R, Wang N, Himpens B, Leybaert L, and Bultynck G (2012) The contractile system as a negative regulator of the connexin 43 hemichannel. Biol Cell 104: 367-377.

Poon IK, Chiu YH, Armstrong AJ, Kinchen JM, Juncadella IJ, Bayliss DA, and Ravichandran KS (2014) Unexpected link between an antibiotic, pannexin channels and apoptosis. Nature 507:329-334.

Prochnow N and Dermietzel R (2008) Connexons and cell adhesion: a romantic phase. Histochem Cell Biol 130:71-77.

Procida K, Jørgensen L, Schmitt N, Delmar M, Taffet SM, Holstein-Rathlou NH, Nielsen MS, and Braunstein TH (2009) Phosphorylation of connexin43 on serine 306 regulates electrical coupling. Heart Rhythm 6:1632-1638.

Puljung MC, Berthoud VM, Beyer EC, and Hanck DA (2004) Polyvalent cations constitute the voltage gating particle in human connexin 37 hemichannels. J Gen Physiol 124:587-603.

Puller C, de Sevilla Müller LP, Janssen-Bienhold U, and Haverkamp S (2009) ZO-1 and the spatial organization of gap junctions and glutamate receptors in the outer plexiform layer of the mammalian retina. J Neurosci 29:6266-6275.

Puranam KL, Laird DW, and Revel JP (1993) Trapping an intermediate form of connexin43 in the Golgi. Exp Cell Res 206:85-92.

Purnick PE, Oh S, Abrams CK, Verselis VK, and Bargiello TA (2000) Reversal of the gating polarity of gap junctions by negative charge substitutions in the $\mathrm{N}$-terminus of connexin 32. Biophys $J$ 79:2403-2415.

Qi Y, Zhao M, Bai Y, Huang L, Yu W, Bian Z, Zhao M, and Li X (2014) Retinal ischemia/reperfusion injury is mediated by Toll-like receptor 4 activation of NLRP3 inflammasomes. Invest Ophthalmol Vis Sci 55:5466-5475.

Qin H, Shao Q, Igdoura SA, Alaoui-Jamali MA, and Laird DW (2003) Lysosomal and proteasomal degradation play distinct roles in the life cycle of $\mathrm{Cx} 43$ in gap junctional intercellular communication-deficient and -competent breast tumor cells. $J$ Biol Chem 278:30005-30014.

Qin J, Zhang G, Zhang X, Tan B, Lv Z, Liu M, Ren H, Qian M, and Du B (2016) TLRActivated gap junction channels protect mice against bacterial infection through extracellular UDP release. J Immunol 196:1790-1798.

Qiu C, Coutinho P, Frank S, Franke S, Law LY, Martin P, Green CR, and Becker DL (2003) Targeting connexin43 expression accelerates the rate of wound repair. Curr Biol 13:1697-1703.

Qiu F and Dahl G (2009) A permeant regulating its permeation pore: inhibition of pannexin 1 channels by ATP. Am J Physiol Cell Physiol 296:C250-C255.

Qu J, Volpicelli FM, Garcia LI, Sandeep N, Zhang J, Márquez-Rosado L, Lampe PD, and Fishman GI (2009) Gap junction remodeling and spironolactonedependent reverse remodeling in the hypertrophied heart. Circ Res 104: $365-371$.

Qu Y, Misaghi S, Newton K, Gilmour LL, Louie S, Cupp JE, Dubyak GR, Hackos D, and Dixit VM (2011) Pannexin-1 is required for ATP release during apoptosis but not for inflammasome activation. J Immunol 186:6553-6561.

Quan XQ, Bai R, Liu N, Chen BD, and Zhang CT (2007) Increasing gap junction coupling reduces transmural dispersion of repolarization and prevents torsade de pointes in rabbit LQT3 model. J Cardiovasc Electrophysiol 18:1184-1189.

Quan XQ, Bai R, Lu JG, Patel C, Liu N, Ruan Y, Chen BD, Ruan L, and Zhang CT (2009) Pharmacological enhancement of cardiac gap junction coupling prevents arrhythmias in canine LQT2 model. Cell Commun Adhes 16:29-38.

Quintanilla RA, Orellana JA, and von Bernhardi R (2012) Understanding risk factors for Alzheimer's disease: interplay of neuroinflammation, connexin-based communication and oxidative stress. Arch Med Res 43:632-644.

Quist AP, Rhee SK, Lin H, and Lal R (2000) Physiological role of gap-junctional hemichannels. Extracellular calcium-dependent isosmotic volume regulation. $J$ Cell Biol 148:1063-1074.

Rackauskas M, Kreuzberg MM, Pranevicius M, Willecke K, Verselis VK, and Bukauskas FF (2007) Gating properties of heterotypic gap junction channels formed of connexins 40, 43, and 45. Biophys $J$ 92:1952-1965.

Radosinska J, Bacova B, Knezl V, Benova T, Zurmanova J, Soukup T, Arnostova P, Slezak J, Gonçalvesova E, and Tribulova N (2013) Dietary omega-3 fatty acids attenuate myocardial arrhythmogenic factors and propensity of the heart to lethal arrhythmias in a rodent model of human essential hypertension. J Hypertens 31: 1876-1885.

Ramachandran S, Xie LH, John SA, Subramaniam S, and Lal R (2007) A novel role for connexin hemichannel in oxidative stress and smoking-induced cell injury. PLoS One 2:e712.

Rami A, Volkmann T, and Winckler J (2001) Effective reduction of neuronal death by inhibiting gap junctional intercellular communication in a rodent model of globa transient cerebral ischemia. Exp Neurol 170:297-304.

Rana S and Dringen R (2007) Gap junction hemichannel-mediated release of glutathione from cultured rat astrocytes. Neurosci Lett 415:45-48.

Rash JE, Kamasawa N, Davidson KG, Yasumura T, Pereda AE, and Nagy JI (2012) Connexin composition in apposed gap junction hemiplaques revealed by matched double-replica freeze-fracture replica immunogold labeling. J Membr Biol 245 : 333-344.

Reaume AG, de Sousa PA, Kulkarni S, Langille BL, Zhu D, Davies TC, Juneja SC, Kidder GM, and Rossant J (1995) Cardiac malformation in neonatal mice lacking connexin43. Science 267:1831-1834.

Rekling JC, Shao XM, and Feldman JL (2000) Electrical coupling and excitatory synaptic transmission between rhythmogenic respiratory neurons in the preBötzinger complex. J Neurosci 20:RC113.

Remo BF, Qu J, Volpicelli FM, Giovannone S, Shin D, Lader J, Liu FY, Zhang J, Lent DS, Morley GE, et al. (2011) Phosphatase-resistant gap junctions inhibit pathological remodeling and prevent arrhythmias. Circ Res 108: 1459-1466 
Ren Q, Riquelme MA, Xu J, Yan X, Nicholson BJ, Gu S, and Jiang JX (2013) Cataract-causing mutation of human connexin 46 impairs gap junction, but increases hemichannel function and cell death. PLoS One 8:e74732.

Ren Y, Zhang CT, Wu J, Ruan YF, Pu J, He L, Wu W, Chen BD, Wang WG, and Wang $\mathrm{L}$ (2006) [The effects of antiarrhythmic peptide AAP10 on ventricular arrhythmias in rabbits with healed myocardial infarction]. Zhonghua Xin Xue Guan Bing Za Zhi 34:825-828.

Retamal MA (2014) Connexin and Pannexin hemichannels are regulated by redox potential. Front Physiol 5:80.

Retamal MA, Cortés CJ, Reuss L, Bennett MV, and Sáez JC (2006) S-nitrosylation and permeation through connexin 43 hemichannels in astrocytes: induction by oxidant stress and reversal by reducing agents. Proc Natl Acad Sci USA 103: $4475-4480$

Retamal MA, Froger N, Palacios-Prado N, Ezan P, Sáez PJ, Sáez JC, and Giaume C (2007a) Cx43 hemichannels and gap junction channels in astrocytes are regulated oppositely by proinflammatory cytokines released from activated microglia. $J$ Neurosci 27:13781-13792.

Retamal MA, Schalper KA, Shoji KF, Bennett MV, and Sáez JC (2007b) Opening of connexin 43 hemichannels is increased by lowering intracellular redox potential. Proc Natl Acad Sci USA 104:8322-8327.

Retamal MA, Reyes EP, García IE, Pinto B, Martínez AD, and González C (2015) Diseases associated with leaky hemichannels. Front Cell Neurosci 9:267.

Revel JP and Karnovsky MJ (1967) Hexagonal array of subunits in intercellular junctions of the mouse heart and liver. J Cell Biol 33:C7-C12.

Rhett JM and Gourdie RG (2012) The perinexus: a new feature of $\mathrm{Cx} 43$ gap junction organization. Heart Rhythm 9:619-623.

Rhett JM, Jourdan J, and Gourdie RG (2011) Connexin 43 connexon to gap junction transition is regulated by zonula occludens-1. Mol Biol Cell 22:1516-1528.

Rhett JM, Veeraraghavan R, Poelzing S, and Gourdie RG (2013) The perinexus: signpost on the path to a new model of cardiac conduction? Trends Cardiovasc Med $\mathbf{2 3}$ $222-228$.

Ribeiro-Rodrigues TM, Catarino S, Marques C, Ferreira JV, Martins-Marques T, Pereira P, and Girão H (2014) AMSH-mediated deubiquitination of Cx43 regulates internalization and degradation of gap junctions. FASEB $J$ 28:4629-4641.

Richards TS, Dunn CA, Carter WG, Usui ML, Olerud JE, and Lampe PD (2004) Protein kinase $\mathrm{C}$ spatially and temporally regulates gap junctional communication during human wound repair via phosphorylation of connexin43 on serine368. $J$ Cell Biol 167:555-562

Riquelme MA and Jiang JX (2013) Elevated intracellular $\mathrm{Ca}^{(2+)}$ signals by oxidative stress activate connexin 43 hemichannels in osteocytes. Bone Res 1:355-361.

Riquelme MA, Kar R, Gu S, and Jiang JX (2013) Antibodies targeting extracellular domain of connexins for studies of hemichannels. Neuropharmacology $\mathbf{7 5}$ : 525-532.

Riquelme MA, Burra S, Kar R, Lampe PD, and Jiang JX (2015) Mitogen-activated protein kinase (MAPK) activated by prostaglandin E2 phosphorylates connexin 43 and closes osteocytic hemichannels in response to continuous flow shear stress. $J$ Biol Chem 290:28321-28328.

Riteau N, Baron L, Villeret B, Guillou N, Savigny F, Ryffel B, Rassendren F, Le Bert M, Gombault A, and Couillin I (2012) ATP release and purinergic signaling: a common pathway for particle-mediated inflammasome activation. Cell Death Dis $\mathbf{3}$ : e403.

Rivedal E and Leithe E (2005) Connexin43 synthesis, phosphorylation, and degradation in regulation of transient inhibition of gap junction intercellular communication by the phorbol ester TPA in rat liver epithelial cells. Exp Cell Res $\mathbf{3 0 2}$ $143-152$.

Robertson J, Lang S, Lambert PA, and Martin PE (2010) Peptidoglycan derived from Staphylococcus epidermidis induces Connexin 43 hemichannel activity with consequences on the innate immune response in endothelial cells. Biochem $J \mathbf{4 3 2}$ $133-143$.

Rodriguez-Sinovas A, Boengler K, Cabestrero A, Gres P, Morente M, Ruiz-Meana M, Konietzka I, Miró E, Totzeck A, Heusch G, et al. (2006) Translocation of connexin 43 to the inner mitochondrial membrane of cardiomyocytes through the heat shock protein 90-dependent TOM pathway and its importance for cardioprotection. Circ Res 99:93-101.

Rodríguez-Sinovas A, Sánchez JA, González-Loyola A, Barba I, Morente M, Aguilar R, Agulló E, Miró-Casas E, Esquerda N, Ruiz-Meana M, et al. (2010) Effects of substitution of $\mathrm{Cx} 43$ by $\mathrm{Cx} 32$ on myocardial energy metabolism, tolerance to ischaemia and preconditioning protection. J Physiol 588:1139-1151.

Rohr S (2004) Role of gap junctions in the propagation of the cardiac action potential. Cardiovasc Res 62:309-322.

Rohr S (2012) Arrhythmogenic implications of fibroblast-myocyte interactions. Circ Arrhythm Electrophysiol 5:442-452.

Rohr S, Kucera JP, Fast VG, and Kléber AG (1997) Paradoxical improvement of impulse conduction in cardiac tissue by partial cellular uncoupling. Science $\mathbf{2 7 5}$ : 841-844.

Rojas Gomez DM, Schulte JS, Mohr FW, and Dhein S (2008) Alpha-1-adrenoceptor subtype selective regulation of connexin 43 expression in rat cardiomyocytes Naunyn Schmiedebergs Arch Pharmacol 377:77-85.

Rook MB, Jongsma HJ, and de Jonge B (1989) Single channel currents of homo- and heterologous gap junctions between cardiac fibroblasts and myocytes. Pflugers Arch 414:95-98.

Roscoe W, Veitch GI, Gong XQ, Pellegrino E, Bai D, McLachlan E, Shao Q, Kidder GM, and Laird DW (2005) Oculodentodigital dysplasia-causing connexin43 mutants are non-functional and exhibit dominant effects on wild-type connexin43. $J$ Biol Chem 280:11458-11466.

Rose B and Loewenstein WR (1975) Permeability of cell junction depends on local cytoplasmic calcium activity. Nature 254:250-252.

Rosenkranz-Weiss P, Tomek RJ, Mathew J, and Eghbali M (1994) Gender-specific differences in expression of mRNAs for functional and structural proteins in rat ventricular myocardium. $J$ Mol Cell Cardiol 26:261-270.
Rossman EI, Liu K, Morgan GA, Swillo RE, Krueger JA, Gardell SJ, Butera J, Gruver M, Kantrowitz J, Feldman HS, et al. (2009) The gap junction modifier, GAP-134 [(2S,4R)-1-(2-aminoacetyl)-4-benzamido-pyrrolidine-2-carboxylic acid], improves conduction and reduces atrial fibrillation/flutter in the canine sterile pericarditis model. J Pharmacol Exp Ther 329:1127-1133.

Rothman SM and Olney JW (1986) Glutamate and the pathophysiology of hypoxicischemic brain damage. Ann Neurol 19:105-111.

Rouach N, Segal M, Koulakoff A, Giaume C, and Avignone E (2003) Carbenoxolone blockade of neuronal network activity in culture is not mediated by an action on gap junctions. $J$ Physiol 553:729-745.

Rouach N, Koulakoff A, Abudara V, Willecke K, and Giaume C (2008) Astroglial metabolic networks sustain hippocampal synaptic transmission. Science 322: $1551-1555$

Rouan F, White TW, Brown N, Taylor AM, Lucke TW, Paul DL, Munro CS, Uitto J, Hodgins MB, and Richard G (2001) trans-dominant inhibition of connexin-43 by mutant connexin-26: implications for dominant connexin disorders affecting epidermal differentiation. J Cell Sci 114:2105-2113.

Rovegno M, Soto PA, Sáez PJ, Naus CC, Sáez JC, and von Bernhardi R (2015) Connexin43 hemichannels mediate secondary cellular damage spread from the trauma zone to distal zones in astrocyte monolayers. Glia 63:1185-1199.

Ruan L, Quan X, Li L, Bai R, Ni M, Xu R, and Zhang C (2014) Increasing gap junction coupling suppresses ibutilide-induced torsades de pointes. Exp Ther Med 7: $1279-1284$.

Ruch RJ, Trosko JE, and Madhukar BV (2001) Inhibition of connexin43 gap junctional intercellular communication by TPA requires ERK activation. J Cell Biochem 83:163-169.

Ruiz-Meana M, Garcia-Dorado D, Lane S, Pina P, Inserte J, Mirabet M, and SolerSoler J (2001) Persistence of gap junction communication during myocardial ischemia. Am J Physiol Heart Circ Physiol 280:H2563-H2571.

Ruiz-Meana M, Núñez E, Miro-Casas E, Martínez-Acedo P, Barba I, RodriguezSinovas A, Inserte J, Fernandez-Sanz C, Hernando V, Vázquez J, et al. (2014) Ischemic preconditioning protects cardiomyocyte mitochondria through mechanisms independent of cytosol. J Mol Cell Cardiol 68:79-88.

Rutledge CA, Ng FS, Sulkin MS, Greener ID, Sergeyenko AM, Liu H, Gemel J, Beyer EC, Sovari AA, Efimov IR, et al. (2014) c-Src kinase inhibition reduces arrhythmia inducibility and connexin43 dysregulation after myocardial infarction. $J$ Am Coll Cardiol 63:928-934.

Sacco RL, Kasner SE, Broderick JP, Caplan LR, Connors JJ, Culebras A, Elkind MS, George MG, Hamdan AD, Higashida RT, et al; American Heart Association Stroke Council, Council on Cardiovascular Surgery and Anesthesia, Council on Cardiovascular Radiology and Intervention, Council on Cardiovascular and Stroke Nursing, Council on Epidemiology and Prevention, Council on Peripheral Vascular Disease, Council on Nutrition, Physical Activity and Metabolism (2013) An updated definition of stroke for the 21st century: a statement for healthcare professionals from the American Heart Association/American Stroke Association. Stroke 44:2064-2089.

Saez JC, Spray DC, Nairn AC, Hertzberg E, Greengard P, and Bennett MV (1986) cAMP increases junctional conductance and stimulates phosphorylation of the 27-kDa principal gap junction polypeptide. Proc Natl Acad Sci USA 83:2473-2477. Sáez JC, Nairn AC, Czernik AJ, Spray DC, Hertzberg EL, Greengard P, and Bennett MV (1990) Phosphorylation of connexin 32, a hepatocyte gap-junction protein, by cAMP-dependent protein kinase, protein kinase $\mathrm{C}$ and $\mathrm{Ca}^{2+} /$ calmodulin-dependent protein kinase II. Eur J Biochem 192:263-273.

Sáez JC, Nairn AC, Czernik AJ, Fishman GI, Spray DC, and Hertzberg EL (1997) Phosphorylation of connexin 43 and the regulation of neonatal rat cardiac myocyte gap junctions. J Mol Cell Cardiol 29:2131-2145.

Saez JC, Berthoud VM, Branes MC, Martinez AD, and Beyer EC (2003) Plasma membrane channels formed by connexins: their regulation and functions. Physiol Rev 83:1359-1400

Sáez JC, Retamal MA, Basilio D, Bukauskas FF, and Bennett MV (2005) Connexinbased gap junction hemichannels: gating mechanisms. Biochim Biophys Acta 1711: $215-224$.

Saffitz JE (2011) Arrhythmogenic cardiomyopathy: advances in diagnosis and disease pathogenesis. Circulation 124:e390-e392.

Saffitz JE and Kléber AG (2004) Effects of mechanical forces and mediators of hypertrophy on remodeling of gap junctions in the heart. Circ Res 94:585-591.

Saffitz JE, Hoyt RH, Luke RA, Lee Kanter H, and Beyer EC (1992) Cardiac myocyte interconnections at gap junctions role in normal and abnormal electrical conduction. Trends Cardiovasc Med 2:56-60.

Saffitz JE, Kanter HL, Green KG, Tolley TK, and Beyer EC (1994) Tissue-specific determinants of anisotropic conduction velocity in canine atrial and ventricular myocardium. Circ Res 74:1065-1070.

Saidi Brikci-Nigassa A, Clement MJ, Ha-Duong T, Adjadj E, Ziani L, Pastre D, Curm PA, and Savarin P (2012) Phosphorylation controls the interaction of the connexin43 C-terminal domain with tubulin and microtubules. Biochemistry 51 4331-4342.

Salameh A (2006) Life cycle of connexins: regulation of connexin synthesis and degradation. Adv Cardiol 42:57-70.

Salameh A and Dhein S (2005) Pharmacology of gap junctions. New pharmacological targets for treatment of arrhythmia, seizure and cancer? Biochim Biophys Acta 1719:36-58.

Salameh A, Schneider P, Mühlberg K, Hagendorff A, Dhein S, and Pfeiffer D (2004) Chronic regulation of the expression of gap junction proteins connexin 40 , connexin43, and connexin 45 in neonatal rat cardiomyocytes. Eur J Pharmacol 503: $9-16$

Salameh A, Frenzel C, Boldt A, Rassler B, Glawe I, Schulte J, Mühlberg K, Zimmer HG, Pfeiffer D, and Dhein S (2006) Subchronic alpha- and beta-adrenergic regulation of cardiac gap junction protein expression. FASEB $J$ 20:365-367.

Salameh A, Krautblatter S, Karl S, Blanke K, Gomez DR, Dhein S, Pfeiffer D, and Janousek $J$ (2009) The signal transduction cascade regulating the expression 
of the gap junction protein connexin 43 by beta-adrenoceptors. Br J Pharmacol 158: 198-208.

Salameh A, Wustmann A, Karl S, Blanke K, Apel D, Rojas-Gomez D, Franke H, Mohr FW, Janousek J, and Dhein S (2010) Cyclic mechanical stretch induces cardiomyocyte orientation and polarization of the gap junction protein connexin43. Circ Res 106:1592-1602.

Salameh A, Djilali H, Blanke K, Gonzalez Casanova J, von Salisch S, Savtschenko A Dhein S, and Dähnert I (2013) Cardiac fibroblasts inhibit $\beta$-adrenoceptordependent connexin43 expression in neonatal rat cardiomyocytes. Naunyn Schmiedebergs Arch Pharmacol 386:421-433.

Salas D, Puebla C, Lampe PD, Lavandero S, and Sáez JC (2015) Role of Akt and $\mathrm{Ca}^{2+}$ on cell permeabilization via connexin 43 hemichannels induced by metabolic inhibition. Biochim Biophys Acta 1852:1268-1277.

Salat-Canela C, Muñoz MJ, Sesé M, Ramón y Cajal S, and Aasen T (2015) Posttranscriptional regulation of connexins. Biochem Soc Trans 43:465-470.

Samie FH and Jalife J (2001) Mechanisms underlying ventricular tachycardia and its transition to ventricular fibrillation in the structurally normal heart. Cardiovasc Res 50:242-250.

Samoilova M, Li J, Pelletier MR, Wentlandt K, Adamchik Y, Naus CC, and Carlen PL (2003) Epileptiform activity in hippocampal slice cultures exposed chronically to bicuculline: increased gap junctional function and expression. J Neurochem $\mathbf{8 6}$ 687-699.

Sánchez JA, Rodríguez-Sinovas A, Barba I, Miró-Casas E, Fernández-Sanz C, RuizMeana M, Alburquerque-Béjar JJ, and García-Dorado D (2013) Activation of RISK and SAFE pathways is not involved in the effects of $\mathrm{Cx} 43$ deficiency on tolerance to ischemia-reperfusion injury and preconditioning protection. Basic Res Cardiol 108 351.

Sandeep TC, Yau JL, MacLullich AM, Noble J, Deary IJ, Walker BR, and Seckl JR (2004) 11Beta-hydroxysteroid dehydrogenase inhibition improves cognitive function in healthy elderly men and type 2 diabetics. Proc Natl Acad Sci USA 101 $6734-6739$.

Sato T, Ohkusa T, Honjo H, Suzuki S, Yoshida MA, Ishiguro YS, Nakagawa $\mathrm{H}$ Yamazaki M, Yano M, Kodama I, et al. (2008) Altered expression of connexin43 contributes to the arrhythmogenic substrate during the development of heart failure in cardiomyopathic hamster. Am J Physiol Heart Circ Physiol 294: H1164-H1173.

Savi M, Bocchi L, Rossi S, Frati C, Graiani G, Lagrasta C, Miragoli M, Di Pasquale E, Stirparo GG, Mastrototaro G, et al. (2016) Antiarrhythmic effect of growth factorsupplemented cardiac progenitor cells in chronic infarcted heart. Am J Physiol Heart Circ Physiol 310:H1622-H1648.

Sawaya SE, Rajawat YS, Rami TG, Szalai G, Price RL, Sivasubramanian N, Mann DL, and Khoury DS (2006) Downregulation of Connexin40 and increased prevalence of atrial arrhythmias in transgenic mice with cardiac restricted overexpression of tumor necrosis factor. Am J Physiol Heart Circ Physiol 292 H1561-H1567.

Sawchuk MA, Hossain MZ, Hertzberg EL, and Nagy JI (1995) In situ transblot and immunocytochemical comparisons of astrocytic connexin- 43 responses to NMDA and kainic acid in rat brain. Brain Res 683:153-157.

Scemes E and Giaume C (2006) Astrocyte calcium waves: what they are and what they do. Glia 54:716-725.

Schalper KA, Palacios-Prado N, Retamal MA, Shoji KF, Martínez AD, and Sáez JC (2008) Connexin hemichannel composition determines the FGF-1-induced membrane permeability and free $\left[\mathrm{Ca}^{2+}\right]$ i responses. Mol Biol Cell 19:3501-3513.

Schalper KA, Sánchez HA, Lee SC, Altenberg GA, Nathanson MH, and Sáez JC (2010) Connexin 43 hemichannels mediate the $\mathrm{Ca}^{2+}$ influx induced by extracellular alkalinization. Am J Physiol Cell Physiol 299:C1504-C1515

Schmilinsky-Fluri G, Valiunas V, Willi M, and Weingart R (1997) Modulation of cardiac gap junctions: the mode of action of arachidonic acid. J Mol Cell Cardiol $\mathbf{2 9}$ 1703-1713.

Schubert AL, Schubert W, Spray DC, and Lisanti MP (2002) Connexin family members target to lipid raft domains and interact with caveolin-1. Biochemistry 41: $5754-5764$

Schulz R, Boengler K, Totzeck A, Luo Y, Garcia-Dorado D, and Heusch G (2007) Connexin 43 in ischemic pre- and postconditioning. Heart Fail Rev 12:261-266.

Schulz R, Görge PM, Görbe A, Ferdinandy P, Lampe PD, and Leybaert L (2015) Connexin 43 is an emerging therapeutic target in ischemia/reperfusion injury, cardioprotection and neuroprotection. Pharmacol Ther 153:90-106.

Schwanke U, Konietzka I, Duschin A, Li X, Schulz R, and Heusch G (2002) No ischemic preconditioning in heterozygous connexin43-deficient mice. Am J Physiol Heart Circ Physiol 283:H1740-H1742.

Schwiebert EM and Zsembery A (2003) Extracellular ATP as a signaling molecule for epithelial cells. Biochim Biophys Acta 1615:7-32.

Seidel T, Salameh A, and Dhein S (2010) A simulation study of cellular hypertrophy and connexin lateralization in cardiac tissue. Biophys $J$ 99:2821-2830.

Seifi M, Fallah S, Ghasemi A, Aghajani H, Razaghi M, and Danaei N (2013) Mutations of the connexin 37 and 40 gap-junction genes in patients with acute myocardial infarction. Clin Lab 59:343-348.

Seki A, Duffy HS, Coombs W, Spray DC, Taffet SM, and Delmar M (2004) Modifications in the biophysical properties of connexin 43 channels by a peptide of the cytoplasmic loop region. Circ Res 95:e22-e28.

Severs NJ, Shovel KS, Slade AM, Powell T, Twist VW, and Green CR (1989) Fate of gap junctions in isolated adult mammalian cardiomyocytes. Circ Res 65:22-42.

Severs NJ, Bruce AF, Dupont E, and Rothery S (2008) Remodelling of gap junctions and connexin expression in diseased myocardium. Cardiovasc Res 80:9-19.

Shah MM, Martinez AM, and Fletcher WH (2002) The connexin43 gap junction protein is phosphorylated by protein kinase A and protein kinase $\mathrm{C}$ : in vivo and in vitro studies. Mol Cell Biochem 238:57-68.

Shan H, Wei J, Zhang M, Lin L, Yan R, Zhang R, and Zhu YH (2015) Suppression of $\mathrm{PKC} \varepsilon$-mediated mitochondrial connexin 43 phosphorylation at serine 368 is involved in myocardial mitochondrial dysfunction in a rat model of dilated cardiomyopathy. Mol Med Rep 11:4720-4726.

Shao Q, Liu Q, Lorentz R, Gong XQ, Bai D, Shaw GS, and Laird DW (2012) Structure and functional studies of N-terminal $\mathrm{Cx} 43$ mutants linked to oculodentodigital dysplasia. Mol Biol Cell 23:3312-3321.

Sharma P, Abbasi C, Lazic S, Teng AC, Wang D, Dubois N, Ignatchenko V, Wong V, Liu J, Araki T, et al. (2015) Evolutionarily conserved intercalated disc protein Tmem65 regulates cardiac conduction and connexin 43 function. Nat Commun 6 : 8391

Shaw RM and Rudy Y (1997a) Electrophysiologic effects of acute myocardial ischemia. A mechanistic investigation of action potential conduction and conduction failure. Circ Res 80:124-138

Shaw RM and Rudy Y (1997b) Ionic mechanisms of propagation in cardiac tissue. Roles of the sodium and L-type calcium currents during reduced excitability and decreased gap junction coupling. Circ Res 81:727-741.

Shaw RM, Fay AJ, Puthenveedu MA, von Zastrow M, Jan YN, and Jan LY (2007) Microtubule plus-end-tracking proteins target gap junctions directly from the cell interior to adherens junctions. Cell 128:547-560.

Shibayama J, Paznekas W, Seki A, Taffet S, Jabs EW, Delmar M, and Musa H (2005) Functional characterization of connexin43 mutations found in patients with oculodentodigital dysplasia. Circ Res 96:e83-e91.

Shibayama J, Gutiérrez C, González D, Kieken F, Seki A, Carrión JR, Sorgen PL, Taffet SM, Barrio LC, and Delmar M (2006a) Effect of charge substitutions at residue his-142 on voltage gating of connexin43 channels. Biophys $J$ 91:4054-4063.

Shibayama J, Lewandowski R, Kieken F, Coombs W, Shah S, Sorgen PL, Taffet SM, and Delmar M (2006b) Identification of a novel peptide that interferes with the chemical regulation of connexin43. Circ Res 98:1365-1372.

Shinotsuka T, Yasui M, and Nuriya M (2014) Astrocytic gap junctional networks suppress cellular damage in an in vitro model of ischemia. Biochem Biophys Res Commun 444:171-176.

Shintani-Ishida K, Uemura K, and Yoshida K (2007) Hemichannels in cardiomyocytes open transiently during ischemia and contribute to reperfusion injury following brief ischemia. Am J Physiol Heart Circ Physiol 293:H1714-H1720.

Shiroshita-Takeshita A, Sakabe M, Haugan K, Hennan JK, and Nattel S (2007) Model-dependent effects of the gap junction conduction-enhancing antiarrhythmic peptide rotigaptide (ZP123) on experimental atrial fibrillation in dogs. Circulation 115:310-318.

Siesjö BK (1992) Pathophysiology and treatment of focal cerebral ischemia. Part I: Pathophysiology. J Neurosurg 77:169-184.

Siller-Jackson AJ, Burra S, Gu S, Xia X, Bonewald LF, Sprague E, and Jiang JX (2008) Adaptation of connexin 43-hemichannel prostaglandin release to mechanical loading. J Biol Chem 283:26374-26382.

Simard M, Arcuino G, Takano T, Liu QS, and Nedergaard M (2003) Signaling at the gliovascular interface. J Neurosci 23:9254-9262.

Simon AM, Goodenough DA, and Paul DL (1998) Mice lacking connexin40 have cardiac conduction abnormalities characteristic of atrioventricular block and bundle branch block. Curr Biol 8:295-298.

Sin WC, Crespin S, and Mesnil M (2012) Opposing roles of connexin43 in glioma progression. Biochim Biophys Acta 1818:2058-2067.

Sin WC, Aftab Q, Bechberger JF, Leung JH, Chen H, and Naus CC (2016) Astrocytes promote glioma invasion via the gap junction protein connexin43. Oncogene $\mathbf{3 5}$ 1504-1516.

Singh D, Solan JL, Taffet SM, Javier R, and Lampe PD (2005) Connexin 43 interacts with zona occludens- 1 and -2 proteins in a cell cycle stage-specific manner. J Biol Chem 280:30416-30421.

Siu RC, Smirnova E, Brown CA, Zoidl C, Spray DC, Donaldson LW, and Zoidl G (2016) Structural and Functional Consequences of Connexin 36 (Cx36) Interaction with Calmodulin. Front Mol Neurosci 9:120.

Siushansian R, Bechberger JF, Cechetto DF, Hachinski VC, and Naus CC (2001) Connexin43 null mutation increases infarct size after stroke. J Comp Neurol 440: 387-394.

Skyschally A, Walter B, Schultz Hansen R, and Heusch G (2013) The antiarrhythmic dipeptide ZP1609 (danegaptide) when given at reperfusion reduces myocardial infarct size in pigs. Naunyn Schmiedebergs Arch Pharmacol 386:383-391.

Smit NW and Coronel R (2014) Stem cells can form gap junctions with cardiac myocytes and exert pro-arrhythmic effects. Front Physiol 5:419.

Smith TD, Mohankumar A, Minogue PJ, Beyer EC, Berthoud VM, and Koval M (2012) Cytoplasmic amino acids within the membrane interface region influence connexin oligomerization. J Membr Biol 245:221-230.

Smith WT IV, Fleet WF, Johnson TA, Engle CL, and Cascio WE (1995) The Ib phase of ventricular arrhythmias in ischemic in situ porcine heart is related to changes in cell-to-cell electrical coupling. Experimental Cardiology Group, University of North Carolina. Circulation 92:3051-3060.

Smyth JW and Shaw RM (2013) Autoregulation of connexin43 gap junction formation by internally translated isoforms. Cell Reports 5:611-618.

Smyth JW, Zhang SS, Sanchez JM, Lamouille S, Vogan JM, Hesketh GG, Hong T, Tomaselli GF, and Shaw RM (2014) A 14-3-3 mode-1 binding motif initiates gap junction internalization during acute cardiac ischemia. Traffic 15:684-699.

Soares AR, Martins-Marques T, Ribeiro-Rodrigues T, Ferreira JV, Catarino S, Pinho MJ, Zuzarte M, Isabel Anjo S, Manadas B, P G Sluijter J, et al. (2015) Gap junctional protein $\mathrm{Cx} 43$ is involved in the communication between extracellular vesicles and mammalian cells. Sci Rep 5:13243.

Soetkamp D, Nguyen TT, Menazza S, Hirschhäuser C, Hendgen-Cotta UB, Rassaf T, Schlüter KD, Boengler K, Murphy E, and Schulz R (2014) S-nitrosation of mitochondrial connexin 43 regulates mitochondrial function. Basic Res Cardiol 109: 433.

Söhl G and Willecke K (2003) An update on connexin genes and their nomenclature in mouse and man. Cell Commun Adhes 10:173-180.

Söhl G and Willecke K (2004) Gap junctions and the connexin protein family. Cardiovasc Res 62:228-232. 
Solan JL and Lampe PD (2007) Key connexin 43 phosphorylation events regulate the gap junction life cycle. $J$ Membr Biol 217:35-41.

Solan JL and Lampe PD (2008) Connexin 43 in LA-25 cells with active v-src is phosphorylated on Y247, Y265, S262, S279/282, and S368 via multiple signaling pathways. Cell Commun Adhes 15:75-84

Solan JL and Lampe PD (2014) Specific Cx43 phosphorylation events regulate gap junction turnover in vivo. FEBS Lett 588:1423-1429.

Solan JL and Lampe PD (2016) Kinase programs spatiotemporally regulate gap junction assembly and disassembly: Effects on wound repair. Semin Cell Dev Biol 50:40-48.

Solan JL, Fry MD, TenBroek EM, and Lampe PD (2003) Connexin43 phosphorylation at $\mathrm{S} 368$ is acute during $\mathrm{S}$ and $\mathrm{G} 2 / \mathrm{M}$ and in response to protein kinase $\mathrm{C}$ activation. $J$ Cell Sci 116:2203-2211.

Solan JL, Marquez-Rosado L, Sorgen PL, Thornton PJ, Gafken PR, and Lampe PD (2007) Phosphorylation at S365 is a gatekeeper event that changes the structure of Cx43 and prevents down-regulation by PKC. J Cell Biol 179:1301-1309.

Sorgen PL, Duffy HS, Sahoo P, Coombs W, Delmar M, and Spray DC (2004) Structural changes in the carboxyl terminus of the gap junction protein connexin 43 indicates signaling between binding domains for c-Src and zonula occludens-1. $J$ Biol Chem 279:54695-54701.

Sosinsky GE and Nicholson BJ (2005) Structural organization of gap junction channels. Biochim Biophys Acta 1711:99-125.

Sosinsky GE, Solan JL, Gaietta GM, Ngan L, Lee GJ, Mackey MR, and Lampe PD (2007) The C-terminus of connexin43 adopts different conformations in the Golgi and gap junction as detected with structure-specific antibodies. Biochem $J \mathbf{4 0 8}$ 375-385.

Sosinsky GE, Boassa D, Dermietzel R, Duffy HS, Laird DW, MacVicar B, Naus CC Penuela S, Scemes E, Spray DC, et al. (2011) Pannexin channels are not gap junction hemichannels. Channels (Austin) 5:193-197.

Sovari AA, Iravanian S, Dolmatova E, Jiao Z, Liu H, Zandieh S, Kumar V, Wang K, Bernstein KE, Bonini MG, et al. (2011) Inhibition of c-Src tyrosine kinase prevents angiotensin II-mediated connexin-43 remodeling and sudden cardiac death. J Am Coll Cardiol 58:2332-2339.

Spach MS and Dolber PC (1990) Discontinuous anisotropic propagation, in Cardiac Electrophysiology: A Textbook (Rosen M, Janse M, and Wit A eds) pp 517-534, Futura Publishing Company Inc., New York.

Spach MS and Heidlage JF (1992) A multidimensional model of cellular effects on the spread of electrotonic currents and on propagating action potentials. Crit Rev Biomed Eng 20:141-169.

Spach MS and Josephson ME (1994) Initiating reentry: the role of nonuniform anisotropy in small circuits. J Cardiovasc Electrophysiol 5:182-209.

Spach MS and Starmer CF (1995) Altering the topology of gap junctions a major therapeutic target for atrial fibrillation. Cardiovasc Res 30:337-344.

Spach MS, Heidlage JF, Dolber PC, and Barr RC (2000) Electrophysiological effects of remodeling cardiac gap junctions and cell size: experimental and model studies of normal cardiac growth. Circ Res 86:302-311.

Spinella F, Rosanò L, Di Castro V, Nicotra MR, Natali PG, and Bagnato A (2003) Endothelin-1 decreases gap junctional intercellular communication by inducing phosphorylation of connexin 43 in human ovarian carcinoma cells. J Biol Chem 278:41294-41301.

Spray DC, Harris AL, and Bennett MV (1981) Gap junctional conductance is a simple and sensitive function of intracellular $\mathrm{pH}$. Science 211:712-715.

Spray DC, Stern JH, Harris AL, and Bennett MV (1982) Gap junctional conductance: comparison of sensitivities to $\mathrm{H}$ and Ca ions. Proc Natl Acad Sci USA $\mathbf{7 9}$ 441-445.

Spray DC, Ye ZC, and Ransom BR (2006) Functional connexin "hemichannels": a critical appraisal. Glia 54:758-773.

Srinivas M and Spray DC (2003) Closure of gap junction channels by arylaminobenzoates. Mol Pharmacol 63:1389-1397.

Srisakuldee W, Jeyaraman MM, Nickel BE, Tanguy S, Jiang ZS, and Kardami E (2009) Phosphorylation of connexin-43 at serine 262 promotes a cardiac injuryresistant state. Cardiovasc Res 83:672-681.

Srisakuldee W, Makazan Z, Nickel BE, Zhang F, Thliveris JA, Pasumarthi KB, and Kardami E (2014) The FGF-2-triggered protection of cardiac subsarcolemma mitochondria from calcium overload is mitochondrial connexin 43-dependent. Cardiovasc Res 103:72-80.

Stables CL, Musa H, Mitra A, Bhushal S, Deo M, Guerrero-Serna G, Mironov S, Zarzoso M, Vikstrom KL, Cawthorn W, et al. (2014) Reduced $\mathrm{Na}^{+}$current density underlies impaired propagation in the diabetic rabbit ventricle. $\mathrm{J} \mathrm{Mol} \mathrm{Cell} \mathrm{Cardiol}$ 69:24-31.

Stauffer BL, Sobus RD, and Sucharov CC (2011) Sex differences in cardiomyocyte connexin43 expression. J Cardiovasc Pharmacol 58:32-39.

Stehberg J, Moraga-Amaro R, Salazar C, Becerra A, Echeverría C, Orellana JA Bultynck G, Ponsaerts R, Leybaert L, Simon F, et al. (2012) Release of gliotransmitters through astroglial connexin 43 hemichannels is necessary for fear memory consolidation in the basolateral amygdala. FASEB $J \mathbf{2 6}$ : 3649-3657.

Stewart MK, Gong XQ, Barr KJ, Bai D, Fishman GI, and Laird DW (2013) The severity of mammary gland developmental defects is linked to the overall functional status of $\mathrm{Cx} 43$ as revealed by genetically modified mice. Biochem J 449 401-413.

Stock A and Sies H (2000) Thyroid hormone receptors bind to an element in the connexin43 promoter. Biol Chem 381:973-979.

Stockert RJ, Spray DC, Gao Y, Suadicani SO, Ripley CR, Novikoff PM, Wolkoff AW, and Hertzberg EL (1999) Deficient assembly and function of gap junctions in Trf1, a trafficking mutant of the human liver-derived cell line HuH-7. Hepatology $\mathbf{3 0}$ $740-747$

Stout CE, Costantin JL, Naus CC, and Charles AC (2002) Intercellular calcium signaling in astrocytes via ATP release through connexin hemichannels. J Biol Chem 277:10482-10488.
Su GY, Wang J, Xu ZX, Qiao XJ, Zhong JQ, and Zhang Y (2015) Effects of rotigaptide (ZP123) on connexin43 remodeling in canine ventricular fibrillation. Mol Med Rep 12:5746-5752.

$\mathrm{Su}$ V and Lau AF (2012) Ubiquitination, intracellular trafficking, and degradation of connexins. Arch Biochem Biophys 524:16-22.

$\mathrm{Su}$ V, Nakagawa R, Koval M, and Lau AF (2010) Ubiquitin-independent proteasomal degradation of endoplasmic reticulum-localized connexin 43 mediated by CIP75. $J$ Biol Chem 285:40979-40990.

Suadicani SO, Flores CE, Urban-Maldonado M, Beelitz M, and Scemes E (2004) Gap junction channels coordinate the propagation of intercellular $\mathrm{Ca}^{2+}$ signals generated by P2Y receptor activation. Glia 48:217-229.

Suadicani SO, Brosnan CF, and Scemes E (2006) P2X7 receptors mediate ATP release and amplification of astrocytic intercellular $\mathrm{Ca}^{2+}$ signaling. $J$ Neurosci 26 : 1378-1385.

Suarez S and Ballmer-Hofer K (2001) VEGF transiently disrupts gap junctional communication in endothelial cells. J Cell Sci 114:1229-1235.

Sun B, Jiang JF, Zhao CM, and Hu CH (2015a) Effects of antiarrhythmic peptide 10 on acute ventricular arrhythmia. Asian Pac J Trop Med 8:229-233.

Sun J, Nguyen T, Aponte AM, Menazza S, Kohr MJ, Roth DM, Patel HH, Murphy E, and Steenbergen C (2015b) Ischaemic preconditioning preferentially increases protein S-nitrosylation in subsarcolemmal mitochondria. Cardiovasc Res 106: 227-236

Sun JM, Wang CM, Guo Z, Hao YY, Xie YJ, Gu J, and Wang AL (2015c) Reduction of isoproterenol-induced cardiac hypertrophy and modulation of myocardial connexin43 by a KATP channel agonist. Mol Med Rep 11:1845-1850.

Sun Y, Yang YQ, Gong XQ, Wang XH, Li RG, Tan HW, Liu X, Fang WY, and Bai D (2013) Novel germline GJA5/connexin40 mutations associated with lone atrial fibrillation impair gap junctional intercellular communication. Hum Mutat 34: 603-609.

Surinkaew S, Kumphune S, Chattipakorn S, and Chattipakorn N (2013) Inhibition of p38 MAPK during ischemia, but not reperfusion, effectively attenuates fatal arrhythmia in ischemia/reperfusion heart. J Cardiovasc Pharmacol 61:133-141.

Swenson KI, Piwnica-Worms H, McNamee H, and Paul DL (1990) Tyrosine phosphorylation of the gap junction protein connexin 43 is required for the pp60v-srcinduced inhibition of communication. Cell Regul 1:989-1002.

Swietach P, Rossini A, Spitzer KW, and Vaughan-Jones RD (2007) $\mathrm{H}^{+}$ion activation and inactivation of the ventricular gap junction: a basis for spatial regulation of intracellular pH. Circ Res 100:1045-1054.

Tabernero A, Gangoso E, Jaraíz-Rodríguez M, and Medina JM (2016) The role of connexin43-Src interaction in astrocytomas: A molecular puzzle. Neuroscience $\mathbf{3 2 3}$ : 183-194

Takemoto Y, Takanari H, Honjo H, Ueda N, Harada M, Kato S, Yamazaki M, Sakuma I, Opthof T, Kodama I, et al. (2012) Inhibition of intercellular coupling stabilizes spiral-wave reentry, whereas enhancement of the coupling destabilizes the reentry in favor of early termination. Am J Physiol Heart Circ Physiol 303: H578-H586.

Takens-Kwak BR and Jongsma HJ (1992) Cardiac gap junctions: three distinct single channel conductances and their modulation by phosphorylating treatments. Pflugers Arch 422:198-200.

Takeuchi H and Suzumura A (2014) Gap junctions and hemichannels composed of connexins: potential therapeutic targets for neurodegenerative diseases. Front Cell Neurosci 8:189.

Takeuchi H, Jin S, Wang J, Zhang G, Kawanokuchi J, Kuno R, Sonobe Y, Mizuno T, and Suzumura A (2006) Tumor necrosis factor-alpha induces neurotoxicity via glutamate release from hemichannels of activated microglia in an autocrine manner. J Biol Chem 281:21362-21368.

Takeuchi H, Mizoguchi H, Doi Y, Jin S, Noda M, Liang J, Li H, Zhou Y, Mori R, Yasuoka S, et al. (2011) Blockade of gap junction hemichannel suppresses disease progression in mouse models of amyotrophic lateral sclerosis and Alzheimer's disease. PLoS One 6: 21108 .

Tamaddon HS, Vaidya D, Simon AM, Paul DL, Jalife J, and Morley GE (2000) Highresolution optical mapping of the right bundle branch in connexin 40 knockout mice reveals slow conduction in the specialized conduction system. Circ Res 87:929-936

Tapia R, Huerta M, Islas S, Avila-Flores A, Lopez-Bayghen E, Weiske J, Huber O and González-Mariscal L (2009) Zona occludens-2 inhibits cyclin D1 expression and cell proliferation and exhibits changes in localization along the cell cycle. Mol Biol Cell 20:1102-1117.

Taylor KA, Wright JR, Vial C, Evans RJ, and Mahaut-Smith MP (2014) Amplification of human platelet activation by surface pannexin-1 channels. $J$ Thromb Haemost 12:987-998.

Tchou GD, Wirka RC, Van Wagoner DR, Barnard J, Chung MK, and Smith JD (2012) Low prevalence of connexin-40 gene variants in atrial tissues and blood from atrial fibrillation subjects. BMC Med Genet 13:102.

TenBroek EM, Lampe PD, Solan JL, Reynhout JK, and Johnson RG (2001) Ser364 of connexin 43 and the upregulation of gap junction assembly by cAMP. $J$ Cell Biol 155:1307-1318

Teunissen BE and Bierhuizen MF (2004) Transcriptional control of myocardial connexins. Cardiovasc Res 62:246-255.

Theodoric N, Bechberger JF, Naus CC, and Sin WC (2012) Role of gap junction protein connexin43 in astrogliosis induced by brain injury. PLoS One 7:e47311.

Thévenin AF, Kowal TJ, Fong JT, Kells RM, Fisher CG, and Falk MM (2013) Proteins and mechanisms regulating gap-junction assembly, internalization, and degradation. Physiology (Bethesda) 28:93-116.

Thibodeau IL, Xu J, Li Q, Liu G, Lam K, Veinot JP, Birnie DH, Jones DL, Krahn AD, Lemery R, et al. (2010) Paradigm of genetic mosaicism and lone atrial fibrillation physiological characterization of a connexin 43-deletion mutant identified from atrial tissue. Circulation 122:236-244.

Thimm J, Mechler A, Lin H, Rhee S, and Lal R (2005) Calcium-dependent open/ closed conformations and interfacial energy maps of reconstituted hemichannels. $J$ Biol Chem 280:10646-10654. 
Thomas MA, Zosso N, Scerri I, Demaurex N, Chanson M, and Staub O (2003) A tyrosine-based sorting signal is involved in connexin43 stability and gap junction turnover. J Cell Sci 116:2213-2222.

Thomas NM, Jasmin JF, Lisanti MP, and Iacobas DA (2011) Sex differences in expression and subcellular localization of heart rhythm determinant proteins. Biochem Biophys Res Commun 406:117-122.

Thomas SA, Schuessler RB, Berul CI, Beardslee MA, Beyer EC, Mendelsohn ME, and Saffitz JE (1998) Disparate effects of deficient expression of connexin43 on atrial and ventricular conduction: evidence for chamber-specific molecular determinants of conduction. Circulation 97:686-691.

Thomas T, Telford D, and Laird DW (2004) Functional domain mapping and selective trans-dominant effects exhibited by $\mathrm{Cx} 26$ disease-causing mutations. J Biol Chem 279:19157-19168.

Thomas T, Jordan K, Simek J, Shao Q, Jedeszko C, Walton P, and Laird DW (2005) Mechanisms of $\mathrm{Cx} 43$ and $\mathrm{Cx} 26$ transport to the plasma membrane and gap junction regeneration. J Cell Sci 118:4451-4462.

Thompson RJ (2015) Pannexin channels and ischaemia. J Physiol 593:3463-3470.

Thrift AG, Dewey HM, Macdonell RA, McNeil JJ, and Donnan GA (2001) Incidence of the major stroke subtypes: initial findings from the North East Melbourne stroke incidence study (NEMESIS). Stroke 32:1732-1738.

Tien T, Muto T, Barrette K, Challyandra L, and Roy S (2014) Downregulation of Connexin 43 promotes vascular cell loss and excess permeability associated with the development of vascular lesions in the diabetic retina. Mol Vis 20:732-741.

Tien T, Muto T, Zhang J, Sohn EH, Mullins RF, and Roy S (2016) Association of reduced Connexin 43 expression with retinal vascular lesions in human diabetic retinopathy. Exp Eye Res 146:103-106.

Tonkin R, Perera C, Lees J, Duffy S, Makker P, O'Carroll S, Nicholson L, Green C, Gorrie C, and Moalem-Taylor G(2016) Blocking connexin-43 hemichannels using Peptide5 reduces pain hypersensitivity following peripheral nerve injury in mice, in Proceedings of the 16th World Congress on Pain, Yokohama, Japan.

Torok K, Stauffer K, and Evans WH (1997) Connexin 32 of gap junctions contains two cytoplasmic calmodulin-binding domains. Biochem $J$ 326:479-483.

Torres A, Wang F, Xu Q, Fujita T, Dobrowolski R, Willecke K, Takano T, and Nedergaard M (2012) Extracellular $\mathrm{Ca}^{2+}$ acts as a mediator of communication from neurons to glia. Sci Signal 5:ra8.

Toth K, Shao Q, Lorentz R, and Laird DW (2010) Decreased levels of Cx43 gap junctions result in ameloblast dysregulation and enamel hypoplasia in Gja1Jrt/+ mice. J Cell Physiol 223:601-609.

Totzeck A, Boengler K, van de Sand A, Konietzka I, Gres P, Garcia-Dorado D, Heusch G, and Schulz R (2008) No impact of protein phosphatases on connexin 43 phosphorylation in ischemic preconditioning. Am J Physiol Heart Circ Physiol 295 H2106-H2112.

Tovar KR, Maher BJ, and Westbrook GL (2009) Direct actions of carbenoxolone on synaptic transmission and neuronal membrane properties. J Neurophysiol 102: 974-978.

Toyofuku T, Yabuki M, Otsu K, Kuzuya T, Hori M, and Tada M (1998) Direct association of the gap junction protein connexin- 43 with $\mathrm{ZO}-1$ in cardiac myocytes. $J$ Biol Chem 273:12725-12731.

Traub O, Eckert R, Lichtenberg-Fraté H, Elfgang C, Bastide B, Scheidtmann KH, Hülser DF, and Willecke K (1994) Immunochemical and electrophysiological characterization of murine connexin 40 and -43 in mouse tissues and transfected human cells. Eur J Cell Biol 64:101-112.

Trexler EB, Bennett MV, Bargiello TA, and Verselis VK (1996) Voltage gating and permeation in a gap junction hemichannel. Proc Natl Acad Sci USA 93:5836-5841.

Trexler EB, Bukauskas FF, Bennett MV, Bargiello TA, and Verselis VK (1999) Rapid and direct effects of $\mathrm{pH}$ on connexins revealed by the connexin46 hemichannel preparation. J Gen Physiol 113:721-742.

Tribulová N, Dupont E, Soukup T, Okruhlicová L, and Severs NJ (2005) Sex differences in connexin-43 expression in left ventricles of aging rats. Physiol Res 54: 705-708.

Trudeau K, Muto T, and Roy S (2012) Downregulation of mitochondrial connexin 43 by high glucose triggers mitochondrial shape change and cytochrome $\mathrm{C}$ release in retinal endothelial cells. Invest Ophthalmol Vis Sci 53:6675-6681.

Tsai CH, Yeh HI, Tian TY, Lee YN, Lu CS, and Ko YS (2004) Down-regulating effect of nicotine on connexin 43 gap junctions in human umbilical vein endothelial cells is attenuated by statins. Eur J Cell Biol 82:589-595.

Tse G and Yeo JM (2015) Conduction abnormalities and ventricular arrhythmogenesis: The roles of sodium channels and gap junctions. Int $J$ Cardiol Heart Vasc 9:75-82.

Tse G, Tse V, and Yeo JM (2016a) Ventricular anti-arrhythmic effects of heptanol in hypokalaemic, Langendorff-perfused mouse hearts. Biomed Rep 4:313-324.

Tse G, Yeo JM, Tse V, Kwan J, and Sun B (2016b) Gap junction inhibition by heptanol increases ventricular arrhythmogenicity by reducing conduction velocity without affecting repolarization properties or myocardial refractoriness in Langendorff-perfused mouse hearts. Mol Med Rep 14:4069-4074.

Tseng WA, Thein T, Kinnunen K, Lashkari K, Gregory MS, D'Amore PA, and Ksander BR (2013) NLRP3 inflammasome activation in retinal pigment epithelial cells by lysosomal destabilization: implications for age-related macular degeneration. Invest Ophthalmol Vis Sci 54:110-120.

Tsui E, Hill KA, Laliberte AM, Paluzzi D, Kisilevsky I, Shao Q, Heathcote JG, Laird DW, Kidder GM, and Hutnik CM (2011) Ocular pathology relevant to glaucoma in a Gja1(Jrt/+) mouse model of human oculodentodigital dysplasia. Invest Ophthalmol Vis Sci 52:3539-3547.

Turner MS, Haywood GA, Andreka P, You L, Martin PE, Evans WH, Webster KA and Bishopric NH (2004) Reversible connexin 43 dephosphorylation during hypoxia and reoxygenation is linked to cellular ATP levels. Circ Res 95:726-733.

Tyagi N, Vacek JC, Givvimani S, Sen U, and Tyagi SC (2010) Cardiac specific deletion of N-methyl-d-aspartate receptor 1 ameliorates mtMMP-9 mediated autophagy/mitophagy in hyperhomocysteinemia. J Recept Signal Transduct Res 30:78-87.
Ueda N, Yamamoto M, Honjo H, Kodama I, and Kamiya K (2014) The role of gap junctions in stretch-induced atrial fibrillation. Cardiovasc Res 104:364-370.

Ujiie H, Chaytor AT, Bakker LM, and Griffith TM (2003) Essential role of Gap junctions in NO- and prostanoid-independent relaxations evoked by acetylcholine in rabbit intracerebral arteries. Stroke 34:544-550.

Unwin PN and Ennis PD (1984) Two configurations of a channel-forming membrane protein. Nature 307:609-613.

Urschel S, Höher T, Schubert T, Alev C, Söhl G, Wörsdörfer P, Asahara T, Dermietzel R, Weiler R, and Willecke K (2006) Protein kinase A-mediated phosphorylation of connexin36 in mouse retina results in decreased gap junctional communication between AII amacrine cells. J Biol Chem 281:33163-33171.

Vaiyapuri S, Jones CI, Sasikumar P, Moraes LA, Munger SJ, Wright JR, Ali MS, Sage T, Kaiser WJ, Tucker KL, et al. (2012) Gap junctions and connexin hemichannels underpin hemostasis and thrombosis. Circulation 125:2479-2491.

Vaiyapuri S, Moraes LA, Sage T, Ali MS, Lewis KR, Mahaut-Smith MP, Oviedo-Orta E, Simon AM, and Gibbins JM (2013) Connexin40 regulates platelet function. Nat Commun 4:2564.

Vaiyapuri S, Flora GD, and Gibbins JM (2015) Gap junctions and connexin hemichannels in the regulation of haemostasis and thrombosis. Biochem Soc Trans 43: 489-494.

Valiunas V, Bukauskas FF, and Weingart R (1997) Conductances and selective permeability of connexin43 gap junction channels examined in neonatal rat heart cells. Circ Res 80:708-719.

van der Velden HM, van Kempen MJ, Wijffels MC, van Zijverden M, Groenewegen WA, Allessie MA, and Jongsma HJ (1998) Altered pattern of connexin40 distribution in persistent atrial fibrillation in the goat. J Cardiovasc Electrophysiol 9: 596-607.

van der Velden HM, Ausma J, Rook MB, Hellemons AJ, van Veen TA, Allessie MA and Jongsma HJ (2000) Gap junctional remodeling in relation to stabilization of atrial fibrillation in the goat. Cardiovasc Res 46:476-486.

Van Kempen MJ, Vermeulen JL, Moorman AF, Gros D, Paul DL, and Lamers WH (1996) Developmental changes of connexin 40 and connexin 43 mRNA distribution patterns in the rat heart. Cardiovasc Res 32:886-900.

Van Norstrand DW, Asimaki A, Rubinos C, Dolmatova E, Srinivas M, Tester DJ, Saffitz JE, Duffy HS, and Ackerman MJ (2012a) Connexin43 mutation causes heterogeneous gap junction loss and sudden infant death. Circulation 125: $474-481$.

Van Norstrand DW, Asimaki A, Rubinos C, Dolmatova E, Srinivas M, Tester DJ, Saffitz JE, Duffy HS, and Ackerman MJ (2012b) Connexin43 mutation causes heterogeneous gap junction loss and sudden infant death. Circulation 125: 474-481.

van Rijen HV, van Kempen MJ, Postma S, and Jongsma HJ (1998) Tumour necrosis factor alpha alters the expression of connexin 43 , connexin40, and connexin37 in human umbilical vein endothelial cells. Cytokine 10:258-264.

van Rijen HV, van Veen TA, Hermans MM, and Jongsma HJ (2000) Human connexin40 gap junction channels are modulated by cAMP. Cardiovasc Res 45: 941-951.

van Rijen HV, van Veen TA, van Kempen MJ, Wilms-Schopman FJ, Potse M, Krueger O, Willecke K, Opthof T, Jongsma HJ, and de Bakker JM (2001) Impaired conduction in the bundle branches of mouse hearts lacking the gap junction protein connexin40. Circulation 103:1591-1598.

VanSlyke JK and Musil LS (2002) Dislocation and degradation from the ER are regulated by cytosolic stress. J Cell Biol 157:381-394.

VanSlyke JK and Musil LS (2005) Cytosolic stress reduces degradation of connexin43 internalized from the cell surface and enhances gap junction formation and function. Mol Biol Cell 16:5247-5257.

van Veen TA, van Rijen HV, and Jongsma HJ (2000) Electrical conductance of mouse connexin45 gap junction channels is modulated by phosphorylation. Cardiovasc Res 46:496-510.

Veenstra RD, Wang HZ, Beyer EC, Ramanan SV, and Brink PR (1994) Connexin37 forms high conductance gap junction channels with subconductance state activity and selective dye and ionic permeabilities. Biophys $J$ 66:1915-1928.

Veenstra RD, Wang HZ, Beblo DA, Chilton MG, Harris AL, Beyer EC, and Brink PR (1995) Selectivity of connexin-specific gap junctions does not correlate with channel conductance. Circ Res 77:1156-1165.

Veeranki S, Givvimani S, Kundu S, Metreveli N, Pushpakumar S, and Tyagi SC (2016) Moderate intensity exercise prevents diabetic cardiomyopathy associated contractile dysfunction through restoration of mitochondrial function and connexin 43 levels in db/db mice. J Mol Cell Cardiol 92:163-173.

Veeraraghavan R, Lin J, Hoeker GS, Keener JP, Gourdie RG, and Poelzing S (2015) Sodium channels in the $\mathrm{Cx} 43$ gap junction perinexus may constitute a cardiac ephapse: an experimental and modeling study. Pflugers Arch 467:2093-2105.

Venance L, Piomelli D, Glowinski J, and Giaume C (1995) Inhibition by anandamide of gap junctions and intercellular calcium signalling in striatal astrocytes. Nature 376:590-594.

Venance L, Rozov A, Blatow M, Burnashev N, Feldmeyer D, and Monyer H (2000) Connexin expression in electrically coupled postnatal rat brain neurons. Proc Natl Acad Sci USA 97:10260-10265.

Verheule S, van Batenburg CA, Coenjaerts FE, Kirchhoff S, Willecke K, and Jongsma HJ (1999) Cardiac conduction abnormalities in mice lacking the gap junction protein connexin40. J Cardiovasc Electrophysiol 10:1380-1389.

Verma V, Larsen BD, Coombs W, Lin X, Spagnol G, Sorgen PL, Taffet SM, and Delmar M (2009) Novel pharmacophores of connexin43 based on the "RXP" series of Cx43-binding peptides. Circ Res 105:176-184.

Verma V, Larsen BD, Coombs W, Lin X, Sarrou E, Taffet SM, and Delmar M (2010) Design and characterization of the first peptidomimetic molecule that prevents acidification-induced closure of cardiac gap junctions. Heart Rhythm 7:1491-1498.

Verselis VK and Srinivas M (2008) Divalent cations regulate connexin hemichannels by modulating intrinsic voltage-dependent gating. J Gen Physiol 132:315-327. 
Verselis VK and Srinivas M (2013) Connexin channel modulators and their mechanisms of action. Neuropharmacology 75:517-524.

Verselis VK, Ginter CS, and Bargiello TA (1994) Opposite voltage gating polarities of two closely related connexins. Nature 368:348-351.

Verselis VK, Trelles MP, Rubinos C, Bargiello TA, and Srinivas M (2009) Loop gating of connexin hemichannels involves movement of pore-lining residues in the first extracellular loop domain. $J$ Biol Chem 284:4484-4493.

Vespa P, Prins M, Ronne-Engstrom E, Caron M, Shalmon E, Hovda DA, Martin NA, and Becker DP (1998) Increase in extracellular glutamate caused by reduced cerebral perfusion pressure and seizures after human traumatic brain injury: a microdialysis study. $J$ Neurosurg 89:971-982.

Vessey DA, Li L, and Kelley M (2011a) Ischemic preconditioning requires opening of pannexin-1/P2X(7) channels not only during preconditioning but again after index ischemia at full reperfusion. Mol Cell Biochem 351:77-84.

Vessey DA, Li L, and Kelley M (2011b) P2X7 receptor agonists pre- and postcondition the heart against ischemia-reperfusion injury by opening pannexin-1/P2X $\mathrm{X}_{7}$ channels. Am J Physiol Heart Circ Physiol 301:H881-H887.

Vessey JP, Lalonde MR, Mizan HA, Welch NC, Kelly ME, and Barnes S (2004) Carbenoxolone inhibition of voltage-gated $\mathrm{Ca}$ channels and synaptic transmission in the retina. $J$ Neurophysiol 92:1252-1256.

Vigmond EJ, Bardakjian BL, Thuneberg L, and Huizinga JD (2000) Intercellular coupling mediated by potassium accumulation in peg-and-socket junctions. IEEE Trans Biomed Eng 47:1576-1583.

Vinken M (2015) Connexin hemichannels: novel mediators of toxicity. Arch Toxicol 89:143-145.

Vinken M (2016) Regulation of connexin signaling by the epigenetic machinery. Biochim Biophys Acta 1859:262-268.

Vinken M, Decrock E, Leybaert L, Bultynck G, Himpens B, Vanhaecke T, and Rogiers $\mathrm{V}$ (2012) Non-channel functions of connexins in cell growth and cell death. Biochim Biophys Acta 1818:2002-2008.

Volkov AG, Paula S, and Deamer DW (1997) Two mechanisms of permeation of small neutral molecules and hydrated ions across phospholipid bilayers. Bioelectrochem Bioenerg 42:153-160.

Wakui S, Yokoo K, Muto T, Suzuki Y, Takahashi H, Furusato M, Hano H, Endou H, and Kanai Y (2006) Localization of Ang-1, -2, Tie-2, and VEGF expression at endothelial-pericyte interdigitation in rat angiogenesis. Lab Invest 86:1172-1184.

Wallraff A, Odermatt B, Willecke K, and Steinhäuser C (2004) Distinct types of astroglial cells in the hippocampus differ in gap junction coupling. Glia 48:36-43.

Wallraff A, Köhling R, Heinemann U, Theis M, Willecke K, and Steinhäuser C (2006) The impact of astrocytic gap junctional coupling on potassium buffering in the hippocampus. J Neurosci 26:5438-5447.

Walrave L, Vinken M, Albertini G, De Bundel D, Leybaert L, and Smolders IJ (2016) Inhibition of connexin 43 hemichannels impairs spatial short-term memory withou affecting spatial working memory. Front Cell Neurosci 10:288.

Walz W and Hertz L (1983) Functional interactions between neurons and astrocytes. II. Potassium homeostasis at the cellular level. Prog Neurobiol 20:133-183.

Wang H, Cao X, Lin Z, Lee M, Jia X, Ren Y, Dai L, Guan L, Zhang J, Lin X, et al. (2015a) Exome sequencing reveals mutation in GJA1 as a cause of keratodermahypotrichosis-leukonychia totalis syndrome. Hum Mol Genet 24:243-250.

Wang H, Hong T, Wang H, and Wang Y (2009) Altered expression of connexin43 and its possible role in endothelin-1-induced contraction in rabbit basilar artery. Neurol Res 31:67-73.

Wang HZ and Veenstra RD (1997) Monovalent ion selectivity sequences of the rat connexin43 gap junction channel. J Gen Physiol 109:491-507.

Wang J, Ma M, Locovei S, Keane RW, and Dahl G (2007a) Modulation of membrane channel currents by gap junction protein mimetic peptides: size matters. $A m$ J Physiol Cell Physiol 293:C1112-C1119.

Wang L, Chen J, Sun Y, Zhang F, Zhu J, Hu S, and Wang DH (2005) Regulation of connexin expression after balloon injury: possible mechanisms for antiproliferative effect of statins. Am J Hypertens 18:1146-1153.

Wang L, Li C, Guo H, Kern TS, Huang K, and Zheng L (2011) Curcumin inhibits neuronal and vascular degeneration in retina after ischemia and reperfusion in jury. PLoS One 6:e23194

Wang N, De Bock M, Antoons G, Gadicherla AK, Bol M, Decrock E, Evans WH, Sipido KR, Bukauskas FF, and Leybaert L (2012a) Connexin mimetic peptides inhibit $\mathrm{Cx} 43$ hemichannel opening triggered by voltage and intracellular $\mathrm{Ca}^{2+}$ elevation. Basic Res Cardiol 107:304.

Wang N, De Bock M, Decrock E, Bol M, Gadicherla A, Bultynck G, and Leybaert L (2013a) Connexin targeting peptides as inhibitors of voltage- and intracellula Ca2+-triggered Cx43 hemichannel opening. Neuropharmacology 75:506-516.

Wang N, De Bock M, Decrock E, Bol M, Gadicherla A, Vinken M, Rogiers V, Bukauskas FF, Bultynck G, and Leybaert L (2013b) Paracrine signaling through plasma membrane hemichannels. Biochim Biophys Acta 1828:35-50.

Wang N, De Vuyst E, Ponsaerts R, Boengler K, Palacios-Prado N, Wauman J, Lai CP De Bock M, Decrock E, Bol M, et al. (2013c) Selective inhibition of Cx43 hemichannels by Gap19 and its impact on myocardial ischemia/reperfusion injury. Basic Res Cardiol 108:309.

Wang N, Sun LY, Zhang SC, Wei R, Xie F, Liu J, Yan Y, Duan MJ, Sun LL, Sun YH, et al. (2015b) MicroRNA-23a participates in estrogen deficiency induced gap junction remodeling of rats by targeting GJA1. Int J Biol Sci 11:390-403.

Wang R, Zhang C, Ruan Y, Liu N, and Wang L (2007b) Changes in phosphorylation of connexin43 in rats during acute myocardial hypoxia and effects of antiarrhythmic peptide on the phosphorylation. J Huazhong Univ Sci Technolog Med Sci 27: 241-244.

Wang X, Arcuino G, Takano T, Lin J, Peng WG, Wan P, Li P, Xu Q, Liu QS, Goldman SA, et al. (2004) P2X7 receptor inhibition improves recovery after spinal cord injury. Nat Med 10:821-827.

Wang XH, Zhuo XZ, Ni YJ, Gong M, Wang TZ, Lu Q, and Ma AQ (2012b) Improvement of cardiac function and reversal of gap junction remodeling by Neuregulin-1 $\beta$ in volume-overloaded rats with heart failure. J Geriatr Cardiol 9:172-179.
Wang Z and Schey KL (2009) Phosphorylation and truncation sites of bovine lens connexin 46 and connexin 50. Exp Eye Res 89:898-904.

Warn-Cramer BJ, Lampe PD, Kurata WE, Kanemitsu MY, Loo LW, Eckhart W, and Lau AF (1996) Characterization of the mitogen-activated protein kinase phosphorylation sites on the connexin-43 gap junction protein. $J$ Biol Chem 271: $3779-3786$

Warn-Cramer BJ, Cottrell GT, Burt JM, and Lau AF (1998) Regulation of connexin-43 gap junctional intercellular communication by mitogen-activated protein kinase. J Biol Chem 273:9188-9196.

Warner A, Clements DK, Parikh S, Evans WH, and DeHaan RL (1995) Specific motifs in the external loops of connexin proteins can determine gap junction formation between chick heart myocytes. J Physiol 488:721-728.

Wasseff SK and Scherer SS (2015) Activated immune response in an inherited leukodystrophy disease caused by the loss of oligodendrocyte gap junctions. Neurobiol Dis 82:86-98.

Watanabe M, Ichinose S, and Sunamori M (2004) Age-related changes in gap junctional protein of the rat heart. Exp Clin Cardiol 9:130-132.

Watkins M, Grimston SK, Norris JY, Guillotin B, Shaw A, Beniash E, and Civitelli R (2011) Osteoblast connexin43 modulates skeletal architecture by regulating both arms of bone remodeling. Mol Biol Cell 22:1240-1251.

Waxse BJ, Sengupta P, Hesketh GG, Lippincott-Schwartz J, and Buss F (2017) Myosin VI facilitates connexin 43 gap junction accretion. J Cell Sci 130: $827-840$

Waza AA, Andrabi K, and Hussain MU (2014) Protein kinase C (PKC) mediated interaction between conexin43 $(\mathrm{Cx} 43)$ and $\mathrm{K}^{(+)}(\mathrm{ATP})$ channel subunit (Kir6.1) in cardiomyocyte mitochondria: Implications in cytoprotection against hypoxia induced cell apoptosis. Cell Signal 26:1909-1917.

Weber PA, Chang HC, Spaeth KE, Nitsche JM, and Nicholson BJ (2004) The permeability of gap junction channels to probes of different size is dependent on connexin composition and permeant-pore affinities. Biophys $J$ 87:958-973

Weilinger NL, Lohman AW, Rakai BD, Ma EM, Bialecki J, Maslieieva V, Rilea T, Bandet MV, Ikuta NT, Scott L, et al. (2016) Metabotropic NMDA receptor signaling couples Src family kinases to pannexin-1 during excitotoxicity. Nat Neurosci 19: $432-442$.

Weingart R and Maurer P (1988) Action potential transfer in cell pairs isolated from adult rat and guinea pig ventricles. Circ Res 63:72-80.

Wen D, Du X, Nie SP, Dong JZ, and Ma CS (2014) Association of Connexin37 C1019T with myocardial infarction and coronary artery disease: a meta-analysis. Exp Gerontol 58:203-207.

Weng S, Lauven M, Schaefer T, Polontchouk L, Grover R, and Dhein S (2002) Pharmacological modification of gap junction coupling by an antiarrhythmic peptide via protein kinase C activation. FASEB $J$ 16:1114-1116.

Wentlandt K, Samoilova M, Carlen PL, and El Beheiry H (2006) General anesthetics inhibit gap junction communication in cultured organotypic hippocampal slices. Anesth Analg 102:1692-1698.

Wetzel U, Boldt A, Lauschke J, Weigl J, Schirdewahn P, Dorszewski A, Doll N, Hindricks G, Dhein S, and Kottkamp H (2005) Expression of connexins 40 and 43 in human left atrium in atrial fibrillation of different aetiologies. Heart $\mathbf{9 1}$ $166-170$

Wilhelm M, Kirste W, Kuly S, Amann K, Neuhuber W, Weyand M, Daniel WG, and Garlichs C (2006) Atrial distribution of connexin 40 and 43 in patients with intermittent, persistent, and postoperative atrial fibrillation. Heart Lung Circ 15: $30-37$.

Willebrords J, Crespo Yanguas S, Maes M, Decrock E, Wang N, Leybaert L, Kwak BR, Green CR, Cogliati B, and Vinken M (2016) Connexins and their channels in inflammation. Crit Rev Biochem Mol Biol 51:413-439.

Wilson CA, Berkowitz BA, Funatsu H, Metrikin DC, Harrison DW, Lam MK and Sonkin PL (1995) Blood-retinal barrier breakdown following experimental retinal ischemia and reperfusion. Exp Eye Res 61:547-557.

Winkler EA, Bell RD, and Zlokovic BV (2011) Central nervous system pericytes in health and disease. Nat Neurosci 14:1398-1405.

Winterhager E, Gellhaus A, Blois SM, Hill LA, Barr KJ, and Kidder GM (2013) Decidual angiogenesis and placental orientation are altered in mice heterozygous for a dominant loss-of-function Gja1 (connexin43) mutation. Biol Reprod 89:111.

Wiśniewski JR, Nagaraj N, Zougman A, Gnad F, and Mann M (2010) Brain phosphoproteome obtained by a FASP-based method reveals plasma membrane protein topology. J Proteome Res 9:3280-3289.

Wit AL and Janse MJ (1992) Experimental models of ventricular tachycardia and fibrillation caused by ischemia and infarction. Circulation 85(1, Suppl);I32-I42.

Wong CW, Burger F, Pelli G, Mach F, and Kwak BR (2003) Dual benefit of reduced $\mathrm{Cx} 43$ on atherosclerosis in LDL receptor-deficient mice. Cell Commun Adhes 10: 395-400.

Wong CW, Christen T, Roth I, Chadjichristos CE, Derouette JP, Foglia BF, Chanson M, Goodenough DA, and Kwak BR (2006) Connexin37 protects against atherosclerosis by regulating monocyte adhesion. Nat Med 12:950-954.

Wu J, McHowat J, Saffitz JE, Yamada KA, and Corr PB (1993) Inhibition of gap junctional conductance by long-chain acylcarnitines and their preferential accumulation in junctional sarcolemma during hypoxia. Circ Res 72:879-889.

Wu T, Handa JT, and Gottsch JD (2005) Light-induced oxidative stress in choroidal endothelial cells in mice. Invest Ophthalmol Vis Sci 46:1117-1123.

Wu X, Huang W, Luo G, and Alain LA (2013) Hypoxia induces connexin 43 dysregulation by modulating matrix metalloproteinases via MAPK signaling. Mol Cell Biochem 384:155-162.

Wu Y, Gu EW, Zhu Y, Zhang L, Liu XQ, and Fang WP (2012) Sufentanil limits the myocardial infarct size by preservation of the phosphorylated connexin 43 . Int Immunopharmacol 13:341-346.

Wu Y, Ma XJ, Wang HJ, Li WC, Chen L, Ma D, and Huang GY (2014) Expression of Cx43-related microRNAs in patients with tetralogy of Fallot. World J Pediatr 10: 138-144. 
Wuestefeld R, Chen J, Meller K, Brand-Saberi B, and Theiss C (2012) Impact of vegf on astrocytes: analysis of gap junctional intercellular communication, proliferation, and motility. Glia 60:936-947.

Xiao D, Chen S, Shao Q, Chen J, Bijian K, Laird DW, and Alaoui-Jamali MA (2014) Dynamin 2 interacts with connexin 26 to regulate its degradation and function in gap junction formation. Int J Biochem Cell Biol 55:288-297.

Xing D, Kjølbye AL, Nielsen MS, Petersen JS, Harlow KW, Holstein-Rathlou NH, and Martins JB (2003) ZP123 increases gap junctional conductance and prevents reentrant ventricular tachycardia during myocardial ischemia in open chest dogs. $J$ Cardiovasc Electrophysiol 14:510-520.

Xu HF, Ding YJ, Shen YW, Xue AM, Xu HM, Luo CL, Li BX, Liu YL, and Zhao ZQ (2012) MicroRNA- 1 represses Cx43 expression in viral myocarditis. Mol Cell Biochem 362:141-148.

Xu H, Gu S, Riquelme MA, Burra S, Callaway D, Cheng H, Guda T, Schmitz J, Fajardo RJ, Werner SL, et al. (2015) Connexin 43 channels are essential for normal bone structure and osteocyte viability. J Bone Miner Res 30:436-448.

Xu Q, Cheong YK, He SQ, Tiwari V, Liu J, Wang Y, Raja SN, Li J, Guan Y, and Li W (2014) Suppression of spinal connexin 43 expression attenuates mechanical hypersensitivity in rats after an L5 spinal nerve injury. Neurosci Lett 566:194-199.

Yan J, Kong W, Zhang Q, Beyer EC, Walcott G, Fast VG, and Ai X (2013) c-Jun N-terminal kinase activation contributes to reduced connexin43 and development of atrial arrhythmias. Cardiovasc Res 97:589-597.

Yancey SB, John SA, Lal R, Austin BJ, and Revel JP (1989) The 43-kD polypeptide of heart gap junctions: immunolocalization, topology, and functional domains. $J$ Cell Biol 108:2241-2254.

Yang B, Lin H, Xiao J, Lu Y, Luo X, Li B, Zhang Y, Xu C, Bai Y, Wang H, et al. (2007) The muscle-specific microRNA miR-1 regulates cardiac arrhythmogenic potential by targeting GJA1 and KCNJ2. Nat Med 13:486-491.

Yang F, Chen WL, Zheng MZ, Yu GW, Xu HJ, Shen YL, and Chen YY (2011) Heat shock protein 90 mediates anti-apoptotic effect of diazoxide by preventing the cleavage of Bid in hypothermic preservation rat hearts. J Heart Lung Transplant 30:928-934.

Yang Y-Q, Liu X, Zhang XL, Wang XH, Tan HW, Shi HF, Jiang WF, and Fang WY (2010) Novel connexin40 missense mutations in patients with familial atrial fibrillation. Europace 12:1421-1427.

Yao JA, Hussain W, Patel P, Peters NS, Boyden PA, and Wit AL (2003) Remodeling of gap junctional channel function in epicardial border zone of healing canine infarcts. Circ Res 92:437-443.

Yasuno S, Kuwahara K, Kinoshita H, Yamada C, Nakagawa Y, Usami S, Kuwabara Y, Ueshima K, Harada M, Nishikimi T, et al. (2013) Angiotensin II type 1a receptor signalling directly contributes to the increased arrhythmogenicity in cardiac hypertrophy. Br J Pharmacol 170:1384-1395.

Ye B, Shen H, Zhang J, Zhu YG, Ransom BR, Chen XC, and Ye ZC (2015) Dual pathways mediate $\beta$-amyloid stimulated glutathione release from astrocytes. Glia 63:2208-2219

Ye ZC, Wyeth MS, Baltan-Tekkok S, and Ransom BR (2003) Functional hemichannels in astrocytes: a novel mechanism of glutamate release. $J$ Neurosci 23 : $3588-3596$.

Yeager M (1998) Structure of cardiac gap junction intercellular channels. J Struct Biol 121:231-245.

Yeager M and Harris AL (2007) Gap junction channel structure in the early 21st century: facts and fantasies. Curr Opin Cell Biol 19:521-528.

Yeh HI, Lu CS, Wu YJ, Chen CC, Hong RC, Ko YS, Shiao MS, Severs NJ, and Tsai $\mathrm{CH}$ (2003) Reduced expression of endothelial connexin37 and connexin40 in hyperlipidemic mice: recovery of connexin37 after 7-day simvastatin treatment. Arterioscler Thromb Vasc Biol 23:1391-1397.

Yeh HI, Lupu F, Dupont E, and Severs NJ (1997) Upregulation of connexin43 gap junctions between smooth muscle cells after balloon catheter injury in the rat carotid artery. Arterioscler Thromb Vasc Biol 17:3174-3184.

Yeh TS, Huang YP, Wang HI, and Pan SL (2016) Spinal cord injury and Parkinson's disease: a population-based, propensity score-matched, longitudinal follow-up study. Spinal Cord 54:1215-1219.

Yi C, Ezan P, Fernández P, Schmitt J, Sáez JC, Giaume C, and Koulakoff A (2017) Inhibition of glial hemichannels by boldine treatment reduces neuronal suffering in a murine model of Alzheimer's disease. Glia DOI: 10.1002/glia.23182 [published ahead of print].

Yi C, Mei X, Ezan P, Mato S, Matias I, Giaume C, and Koulakoff A (2016) Astroglial connexin43 contributes to neuronal suffering in a mouse model of Alzheimer's disease. Cell Death Differ 23:1691-1701.

Yin X, Jedrzejewski PT, and Jiang JX (2000) Casein kinase II phosphorylates lens connexin 45.6 and is involved in its degradation. $J$ Biol Chem 275:6850-6856.

Yin X, Gu S, and Jiang JX (2001) The development-associated cleavage of lens connexin 45.6 by caspase-3-like protease is regulated by casein kinase II-mediated phosphorylation. J Biol Chem 276:34567-34572.

Yogo K, Ogawa T, Akiyama M, Ishida N, and Takeya T (2002) Identification and functional analysis of novel phosphorylation sites in $\mathrm{Cx} 43$ in rat primary granulosa cells. FEBS Lett 531:132-136.
Yogo K, Ogawa T, Akiyama M, Ishida-Kitagawa N, Sasada H, Sato E, and Takeya T (2006) PKA implicated in the phosphorylation of $\mathrm{Cx} 43$ induced by stimulation with FSH in rat granulosa cells. J Reprod Dev 52:321-328.

Young EJ, Hill MA, Wiehler WB, Triggle CR, and Reid JJ (2008) Reduced EDHF responses and connexin activity in mesenteric arteries from the insulin-resistant obese Zucker rat. Diabetologia 51:872-881.

Young RC, Schumann R, and Zhang P (2002) The signaling mechanisms of long distance intercellular calcium waves (far waves) in cultured human uterine myocytes. J Muscle Res Cell Motil 23:279-284.

Yu J, Bippes CA, Hand GM, Muller DJ, and Sosinsky GE (2007) Aminosulfonate modulated $\mathrm{pH}$-induced conformational changes in connexin26 hemichannels. $J$ Biol Chem 282:8895-8904.

Yu W, Dahl G, and Werner R (1994) The connexin43 gene is responsive to oestrogen. Proc Biol Sci 255:125-132.

Yulyana Y, Endaya BB, Ng WH, Guo CM, Hui KM, Lam PY, and Ho IA (2013) Carbenoxolone enhances TRAIL-induced apoptosis through the upregulation of death receptor 5 and inhibition of gap junction intercellular communication in human glioma. Stem Cells Dev 22:1870-1882.

Zahraoui A (2004) [Tight junctions, a platform regulating cell proliferation and polarity]. Med Sci (Paris) 20:580-585.

Zampighi GA, Loo DD, Kreman M, Eskandari S, and Wright EM (1999) Functional and morphological correlates of connexin50 expressed in Xenopus laevis oocytes. $J$ Gen Physiol 113:507-524.

Zappitelli T, Chen F, and Aubin JE (2015) Up-regulation of BMP2/4 signaling increases both osteoblast-specific marker expression and bone marrow adipogenesis in Gja1Jrt/+ stromal cell cultures. Mol Biol Cell 26:832-842.

Zenaro E, Piacentino G, and Constantin G (2016) The blood-brain barrier in Alzheimer's disease. Neurobiol Dis DOI: 10.1016/j.nbd.2016.07.007 [published ahead of print].

Zendedel A, Johann S, Mehrabi S, Joghataei MT, Hassanzadeh G, Kipp M, and Beyer C (2016) Activation and Regulation of NLRP3 Inflammasome by Intrathecal Application of SDF-1a in a Spinal Cord Injury Model. Mol Neurobiol 53:3063-3075.

Zhang D, Zhang Y, Zhao C, Zhang W, Shao G, and Zhang H (2016a) Effect of lysophosphatidic acid on the immune inflammatory response and the connexin 43 protein in myocardial infarction. Exp Ther Med 11:1617-1624

Zhang FF, Morioka N, Kitamura T, Fujii S, Miyauchi K, Nakamura Y, HisaokaNakashima K, and Nakata Y (2016b) Lycopene ameliorates neuropathic pain by upregulating spinal astrocytic connexin 43 expression. Life Sci 155:116-122.

Zhang J, O'Carroll SJ, Wu A, Nicholson LF, and Green CR (2010) A model for ex vivo spinal cord segment culture-a tool for analysis of injury repair strategies. $J \mathrm{Neu}$ rosci Methods 192:49-57.

Zhang W, Zhao G, Hu X, Wang M, Li H, Ye Y, Du Q, Yao J, Bao Z, Hong W, et al. (2014) Aliskiren-attenuated myocardium apoptosis via regulation of autophagy and connexin-43 in aged spontaneously hypertensive rats. J Cell Mol Med 18 $1247-1256$

Zhang XS and Xiang BR (2009) Discontinued drugs in 2008: cardiovascular drugs. Expert Opin Investig Drugs 18:875-885.

Zhang YW, Kaneda M, and Morita I (2003) The gap junction-independent tumorsuppressing effect of connexin 43. J Biol Chem 278:44852-44856.

Zhao LL, Chen HJ, Chen JZ, Yu M, Ni YL, and Zhang WF (2008) Losartan reduced connexin43 expression in left ventricular myocardium of spontaneously hypertensive rats. J Zhejiang Univ Sci B 9:448-454.

Zhao X, Tang X, Ma T, Ding M, Bian L, Chen D, Li Y, Wang L, Zhuang Y, Xie M, et al. (2015) Levonorgestrel inhibits human endometrial cell proliferation through the upregulation of gap junctional intercellular communication via the nuclear translocation of Ser255 phosphorylated Cx43. BioMed Res Int 2015 758684 .

Zheng L, Gong B, Hatala DA, and Kern TS (2007) Retinal ischemia and reperfusion causes capillary degeneration: similarities to diabetes. Invest Ophthalmol Vis Sci 48:361-367.

Zhou JZ and Jiang JX (2014) Gap junction and hemichannel-independent actions of connexins on cell and tissue functions-an update. FEBS Lett 588:1186-1192.

Zhou K, Shi L, Wang Y, Chen S, and Zhang J (2016) Recent Advances of the NLRP3 Inflammasome in Central Nervous System Disorders. J Immunol Res 2016: 9238290.

Zhou L, Kasperek EM, and Nicholson BJ (1999) Dissection of the molecular basis of pp60(v-src) induced gating of connexin 43 gap junction channels. J Cell Biol 144 1033-1045.

Zhou Q, Wang TJ, Zhang CT, Ruan L, Li LD, Xu RD, Quan XQ, and Ni MK (2011) [Effect of antiarrhythmic peptide on ventricular arrhythmia induced by lysophosphatidic acid]. Zhonghua Xin Xue Guan Bing Za Zhi 39:301-304.

Zhou Y, Yang W, Lurtz MM, Ye Y, Huang Y, Lee HW, Chen Y, Louis CF, and Yang JJ (2007) Identification of the calmodulin binding domain of connexin 43. J Biol Chem 282:35005-35017.

Zhuang J, Yamada KA, Saffitz JE, and Kléber AG (2000) Pulsatile stretch remodels cell-to-cell communication in cultured myocytes. Circ Res 87:316-322. 